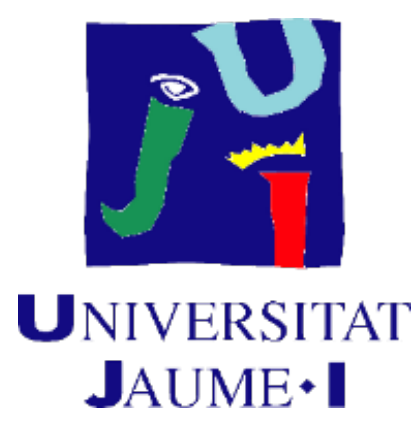

Doctoral School of Universitat Jaume I

Doctoral Programme in Industrial Technologies and Materials

\title{
Event-Based PID Controllers With Fixed Threshold SAmpling Strategies
}

A dissertation submitted by Oscar Miguel Escrig to obtain the degree of Doctor of Philosophy from the Universitat Jaume I

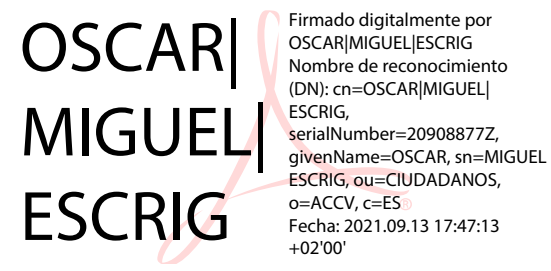

AUTHOR

Oscar Miguel Escrig

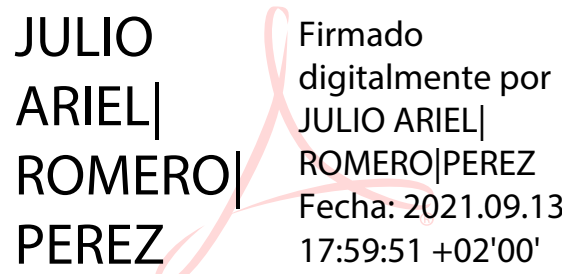

ADVISOR

Dr. Julio Ariel Romero Pérez 



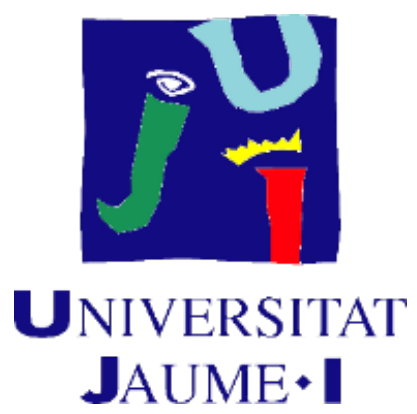

Escuela de Doctorado de la Universitat Jaume I

Programa de Doctorado en Tecnologías Industriales y Materiales

\section{Controladores PID Basados en Eventos GENERADOS MEDIANTE ESTRATEGIAS DE MUESTREO CON UMBRALES FIJOS}

Memoria presentada por Oscar Miguel Escrig para optar al grado de doctor por la Universitat Jaume I

AUTOR

Oscar Miguel Escrig
DIRECTOR

Dr. Julio Ariel Romero Pérez 



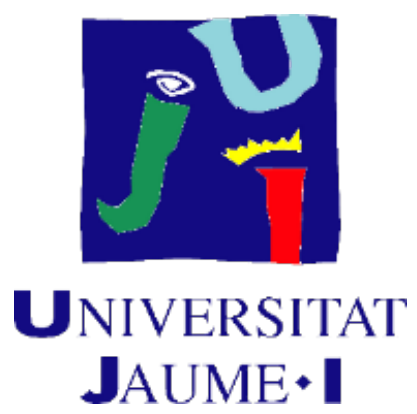

Escola de Doctorat de la Universitat Jaume I Programa de doctorat en Tecnologies Industrials i Materials

\section{Controladors PID BAsats EN ESDEVENIMENTS GENERATS PER ESTRATÈGIES DE MOSTREIG AMB LLINDARS FIXES}

Memòria presentada per Oscar Miguel Escrig per a optar al grau de doctor per la Universitat Jaume I

AUTOR

Oscar Miguel Escrig
DIRECTOR

Dr. Julio Ariel Romero Pérez 

Als meus pares 



\section{Funding}

\section{Contrato Predoctoral}

Beca para la contratación de personal investigador de carácter predoctoral del Programa Operativo del Fondo Social Europeo 2014-2020 de la Comunitat Valenciana. (Ref. ACIF/2018/244)

\section{Participación en proyectos}

Proyecto de Investigación "Adaptación de algoritmos de control PID al contexto de la Industria 4.0" de la Universitat Jaume I (Ref. UJI-B2018-39)

Proyecto de Investigación "Desarrollo de algoritmos de control para sistemas distribuidos implementables mediante estándares industriales" de la Universitat Jaume I (Ref. P11B2015-42)

Proyecto de Investigación "Estrategias de estimación y control para la minimización del coste energético en procesos con perturbaciones variantes y sujetos a periodos tarifarios" del Ministerio de Ciencia e Innovación (Ref. TEC2015-69155-R)

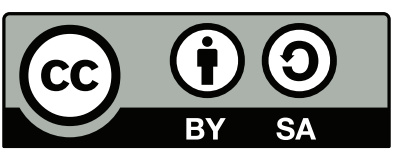





\section{Compendium of publications}

1. Oscar Miguel-Escrig \& Julio-Ariel Romero-Pérez (2021). Event-based discrete PI controllers robustness analysis through Sampled Describing Function technique. International Journal of Control. DOI: https://doi.org/10.1080/00207179.2021.1962980. Impact factor: 2.888

2. Oscar Miguel-Escrig \& Julio-Ariel Romero-Pérez (2021). Tuning procedure for event-based PI controllers under regular quantization with hysteresis. Journal of the Franklin Institute. DOI: https://doi.org/10.1016/j.jfranklin.2021.04.004. Impact factor: 4.504

3. Oscar Miguel-Escrig \& Julio-Ariel Romero-Pérez (2020). Regular quantisation with hysteresis: a new sampling strategy for event-based PID control systems. IET Control Theory \& Applications, 14(15), 2163-2175. DOI: 10.1049/iet-cta.2020.0128. Impact factor: 3.343

4. Oscar Miguel-Escrig, Julio-Ariel Romero-Pérez \& Roberto Sanchis-Llopis (2020). Tuning PID controllers with symmetric-send-on-delta sampling strategy. Journal of the Franklin Institute, 357(2), 832-862. DOI: https://doi.org/10.1016/j.jfranklin.2019.10.008. Impact factor: 4.504

This thesis has been accepted by the co-authors of the publications listed above that have waved the right to present them as a part of another $\mathrm{PhD}$ thesis. 



\section{Abstract}

Event-Based Control (EBC) has emerged in recent years as a solid alternative to classical control techniques for controlling continuous system. This technique decreases the amount of information needed to perform the control without degrading significantly the performance of the loop and diminishes the average computational cost of the algorithms. Therefore, its application is specially indicated for networked control system, in which the data flow between devices must be optimized. A lot of EBC schemes and controllers have been proposed, being the most relevant schemes those based on the quantization of the error signal and being the Proportional-IntegralDerivative (PID) controllers the most spread controllers in industry. However, despite all the research effort invested in these new proposals, not so much has been spent on characterizing their robustness and performance.

This thesis proposes a new EBC scheme as a generalization of two of the most used, the Symmetric-Send-On-Delta (SSOD) and the Regular Quantization (RQ), named Regular Quantification with Hysteresis $(\mathrm{RQH})$. The robustness against limit cycle oscillations that can be induced by this new scheme is characterized using the Describing Function (DF) technique, which has been used to develop some robustness measures in terms of gain and phase as classical control robustness measures.

Thanks to the defined robustness measures, a tuning method is developed for PI controllers under an RQH sampling strategy. The proposed tuning method uses both the new specific and the classical robustness measures to ensure stable responses. The proposed method is compared to classical tuning methods used for EBC control, showing an improvement in robustness and performance.

This thesis also addresses the robustness issues arisen by the SSOD, fully characterizing its robustness through the application of the Tsypkin method, which allows an exact analysis of the system. Thanks to the development of this analysis, a tuning method for PID controllers under this sampling strategy is proposed, which takes also into account the amplification of the controller as a design parameter. The methodology is shown to be valid also to evaluate the robustness of controllers under an RQH sampling strategy.

In addition, this thesis provides the resources and a methodology to study any kind of sampling scheme with a fixed threshold input-output relation. The study is based on a technique which allows predicting biased oscillations and sheds light on some of the initial conditions required to propitiate limit cycle oscillations on some samplers. 
This thesis also addresses the issues derived from a real implementation of the sampling strategy and the controllers, which is done mainly in processor-based devices. Therefore, a theoretical analysis study is provided in which the effect of the discrete implementation of PI controllers in SSOD loops is studied. A robustness measure is developed to characterize the robustness against limit cycle oscillations induced by SSOD sampling with a discrete PI controller.

The implementation of EBC controllers is also treated in this thesis considering standard IEC 61499 as the programming language. An application for controlling a networked control system in this programming standard has been developed in which the RQH quantization has been implemented. The application and the different tuning rules and analysis techniques developed in this thesis have been applied to a concrete case. The results obtained from the experiments carried out with a real system validate the proposed theoretical analysis for its application to the study of networked control systems. 


\section{Resumen}

El control basado en eventos (EBC, del inglés Event-Based Control) ha emergido en los últimos años como una alternativa sólida a las técnicas de control clásicas para el control de sistemas continuos. Esta técnica disminuye la cantidad de información necesaria para realizar el control sin perjudicar significativamente el desempeño del lazo de control y reduce el coste computacional medio de los algoritmos de control. Por estos motivos, su aplicación está especialmente indicada para los sistemas de control en red, en los cuales la transmisión de datos entre dispositivos debe ser óptima. Se han propuesto muchos esquemas y controladores que se ajustan a los principios del EBC, siendo los esquemas más relevantes aquellos basados en la cuantificación de la señal de error y siendo los controladores de tipo Proporcional-Integral-Derivativo (PID) los más extendidos en la industria. Sin embargo, a pesar de todo el esfuerzo de investigación empleado en el desarrollo de estas propuestas, no se ha invertido tanto en caracterizar de forma precisa su robustez y desempeño.

En esta tesis se propone un nuevo esquema de muestreo para el EBC que supone una generalización de dos de los esquemas más usados, el envío por cruce de niveles simétricos (SSOD, del inglés Symmetric-Send-On-Delta) y la cuantificación regular (RQ, del inglés Regular Quantization), a la que se ha llamado cuantificación regular con histéresis (RQH, del inglés Regular Quantization with Hysteresis). Se ha caracterizado la robustez frente a la aparición de oscilaciones de ciclo límite que pueden ser inducidas por este esquema usando la técnica de la función descriptiva, la cual se ha usado para desarrollar medidas de robustez específicas definidas en términos de margen de ganancia y de fase tal y como se define la robustez con márgenes clásicos.

Gracias a dichos márgenes de robustez, se ha desarrollado un método para sintonizar controladores PI bajo un esquema de muestreo RQH. El método propuesto usa tanto medidas de robustez clásicas como específicas para garantizar repuestas estables. Este método se ha comparado con métodos tradicionales de sintonizado de controladores normalmente aplicados para el control EBC, mostrando una mejora en la robustez obtenida y en el desempeño.

La tesis también estudia los problemas de robustez derivados de la aplicación de un muestreo SSOD, caracterizando por completo su robustez mediante la aplicación del método de Tsypkin, el cual permite un análisis exacto del sistema. Gracias al desarrollo de este análisis, se ha propuesto un método para la sintonización de controladores PID bajo esta técnica de muestreo, 
que tiene en cuenta la amplificación de ruido del controlador como parámetro de diseño. El método de análisis se demuestra válido para analizar también la robustez de controladores bajo un esquema de muestreo RQH.

Además, esta tesis provee los recursos y métodos necesarios para estudiar cualquier tipo de esquema de muestreo con niveles fijos de muestreo y de cuantificación. El estudio se basa en una técnica que permite predecir oscilaciones de ciclo límites con componente de continua y arroja luz sobre algunas de las condiciones iniciales que se requieren para la aparición de ciclos límite en diferentes muestreadores.

Esta tesis también trata los problemas derivados de la implementación real de las estrategias de muestreo estudiadas y de los controladores, los cuales son implementados mayoritariamente por dispositivos con procesador. Por lo tanto, se presenta un análisis teórico sobre el efecto en la robustez de la implementación discreta de controladores PI en bucles de control con SSOD. Se ha desarrollado una medida de robustez específica ante la aparición de ciclos límite inducidos por el SSOD en bucles con un PI discreto.

La implementación de controladores EBC también se trata en esta tesis, considerando el estándar industrial IEC 61499 como lenguaje de programación. Se ha desarrollado una aplicación para controlar sistemas distribuidos en red en dicho estándar, en la cual la cuantificación RQH ha sido implementada. La aplicación y las diferentes reglas de sintonía y análisis desarrolladas en la tesis han sido aplicadas a un caso concreto. Los resultados obtenidos de los experimentos realizados sobre un sistema real acreditan los análisis teóricos propuestos para su aplicación al estudio de sistema de control en red. 


\section{Resum}

El control basat en esdeveniments (EBC, de l'anglès Event-Based Control) ha sorgit en els últims anys com una alternativa sòlida a les tècniques de control clàssiques per al control de sistemes continus. Aquesta tècnica disminueix la quantitat d'informació necessària per realitzar el control sense perjudicar significativament el rendiment del bucle de control i redueix el cost computacional mitjà dels algoritmes de control. Per aquests motius, la seua aplicació està especialment indicada per als sistemes de control en xarxa, en els quals la transmissió de dades entre dispositius deu ser òptima. S'han proposat molts esquemes i controladors que s'ajusten als principis de l'EBC, sent el esquemes més rellevants aquells basats en la quantificació del senyal d'error i sent els controladors de tipus Proporcional-Integral-Derivatiu (PID) els més estesos en la industria. No obstant això, malgrat tot l'esforç d'investigació dedicat al desenvolupament d'aquestes propostes, no se n'ha invertit tant en caracteritzar de forma precisa la seua robustesa i rendiment.

En aquesta tesi es proposa un nou esquema de mostreig per a l'EBC que suposa una generalització de dos dels esquemes més emprats, l'enviament per creuament de nivells simètrics (SSOD, de l'anglès Symmetric-Send-On-Delta) i la quantificació regular (RQ, de l'anglès Regular Quantization), a la que s'ha anomenat quantificació regular amb histèresi (RQH, de l'anglès Regular Quantization with Hysteresis). La robustesa front a l'aparició d'oscil · lacions de cicle limit que poden ser induïdes per aquest esquema s'han caracteritzat amb la tècnica de la funció descriptiva, la qual s'ha emprat per a desenvolupar mesures de robustesa específiques definides en termes de màrgens de guany i de fase, tal i com es defineix la robustesa amb els màrgens clàssics.

Gràcies a dits màrgens de robustesa, s'ha desenvolupat un mètode per sintonitzar controladors PI sota un esquema de mostreig RQH. El mètode proposat empra tant mesures de robustesa clàssiques com específiques per a garantir respostes estables. Aquest mètode s'ha comparat amb mètodes tradicionals de sintonització de controladors normalment aplicats per al control EBC, mostrant una millora en la robustesa obtinguda i en el rendiment.

La tesi també estudia els problemes de robustesa derivats de l'aplicació d'un mostreig SSOD, caracteritzant per complet la seua robustesa mitjançant l'aplicació del mètode de Tsypkin, el qual permet una anàlisi exacta del sistema. Gràcies al desenvolupament d'aquesta anàlisi, s'ha proposat un mètode per a la sintonització de controladors PID aplicats a aquesta tècnica de 
mostreig, el qual té en compte l'amplificació del soroll del controlador com a paràmetre de disseny. El mètode d'anàlisi es demostra vàlid per analitzar també la robustesa de controladors sota un esquema de mostreig RQH.

A més, aquesta tesi proveu els recursos i mètodes necessaris per estudiar qualsevol tipus d'esquema de mostreig amb nivells fixos de mostreig i de quantificació. L'estudi es basa en una tècnica que permet predir oscil · lacions de cicle limit amb component de continua i esclareix algunes de les condicions inicials que es requereixen per a l'aparició de cicles limit en diferents mostrejadors.

Aquesta tesi també tracta els problemes derivats de la implementació real de les estratègies de mostreig estudiades i dels controladors, els quals són implementats majoritàriament en dispositius amb processador. Per tant, es presenta una anàlisi teòrica sobre l'efecte en la robustesa de la implementació discreta de controladors PI en bucles de control amb SSOD. S'ha desenvolupat una mesura de robustesa específica front a l'aparició de cicles limit induïts per l'SSOD en bucles amb un PI discret.

La implementació de controladors EBC també es tracta en aquesta tesi, considerant l'estàndard industrial IEC 61499 com a llenguatge de programació. S'ha desenvolupat una aplicació per a controlar sistemes distribuïts en xarxa en aquest estàndard, en el qual la quantificació RQH ha sigut implementada. L'aplicació i les diferents regles de sintonia i anàlisis desenvolupades en la tesi han sigut aplicats a un cas concret. Els resultats obtinguts dels experiments realitzats sobre un sistema real acrediten les anàlisis teòriques proposades per a la seua aplicació a l'estudi de sistemes de control en xarxa. 


\section{Acknowledgments}

En primer lloc, vull agrair als meus pares tot el que han fet per mi, sempre han estat quan m'han fet falta per tal que jo poguera arribar on me proposara. També agrair a ma wela Rosa que des de menudet m'ha criat i que aconsegueix traure'm sempre un somriure. I una menció especial als que heu marxat, vos duc ben dins.

En segon lloc, a Raquel, per totes aquelles coses que fas i que no t'agraïsc lo suficient, amb tu és tot més fàcil. T'estime preciosa.

En tercer lloc, a tots els companys amb qui treballe colze amb colze i compartim tant els moments dolços com els amargs. Esmentar especialment a Ester, Carlos i Ruben, persones molt peculiars i excepcionals que tantes alegries m'han donat. I evidentment a Julio, que abans que el meu director de tesi és una gran persona i amic.

Finalment, agrair també als meus amics, els de sempre, aqueixos que no importa el que passe ni quant de temps fa que no ens veiem sempre van a estar. Als 30 o als que siguen, però seguim caminant. 



\section{Contents}

Funding $\quad$ I

$\begin{array}{ll}\text { Compendium of publications } & \text { III }\end{array}$

$\begin{array}{ll}\text { Abstract } & \text { V }\end{array}$

$\begin{array}{ll}\text { Acknowledgments } & \text { XI }\end{array}$

$\begin{array}{ll}\text { Notation } & \text { XXVII }\end{array}$

1 Introduction 1

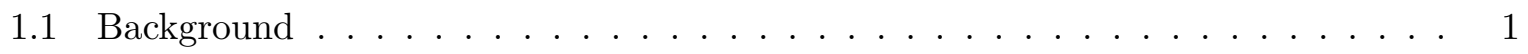

1.1.1 Implementation of EBC in the standard IEC $61499 \ldots \ldots \ldots$

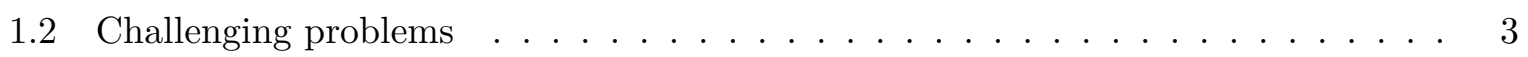

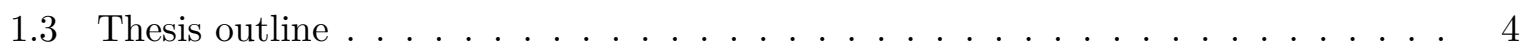

$\begin{array}{lll}2 & \text { RQH sampling strategy analysis with DF method } & 7\end{array}$

2.1 Introduction . . . . . . . . . . . . . . . . . . . . . . . . 7

2.2 Problem statement . . . . . . . . . . . . . . . . . . . . . 10

$2.3 \mathrm{RQH}$ sampling strategy $\ldots \ldots \ldots \ldots \ldots \ldots \ldots \ldots$

2.3 .1 Event generation . . . . . . . . . . . . . . . . . . . 12

2.4 Robustness to oscillation induced by $\mathrm{RQH} \ldots \ldots \ldots \ldots \ldots \ldots$

2.4.1 Network communication delay influence on the robustness margins . . . . 18

2.5 Evaluation of classical tuning methods using the proposed margins . . . . . . . . 19

2.5.1 Influence of $h / \delta$ on the robustness margins . . . . . . . . . . . 21

2.5.2 Influence of controller's parameters on the robustness margins . . . . . . . 22

2.6 Simulation examples . . . . . . . . . . . . . . . . . . . . . . . . 23

2.7 Conclusions . . . . . . . . . . . . . . . . . . . . . . . . . . 29 
3 Tuning Procedure for PI controllers under RQH sampling 31

3.1 Introduction . . . . . . . . . . . . . . . . . . . . . . 31

3.2 Problem statement . . . . . . . . . . . . . . . . . . . 34

3.3 Tuning procedure for RQH based PI . . . . . . . . . . . . . . . . . 37

3.4 Design validation . . . . . . . . . . . . . . . . . . . . . 49

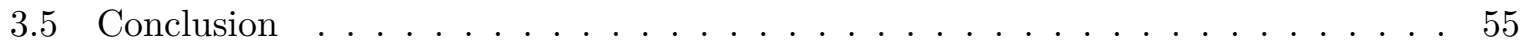

4 Analysis and tuning of SSOD and RQH systems with Tsypkin method 57

4.1 Introduction . . . . . . . . . . . . . . . . . . . . . . 57

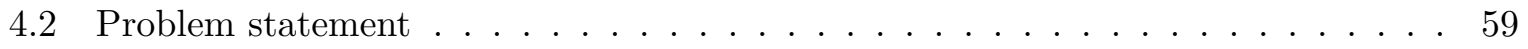

4.3 New robustness margin for existence of limit cycles . . . . . . . . . . . . . . 61

4.3.1 Tsypkin's method approach . . . . . . . . . . . . . . . . 61

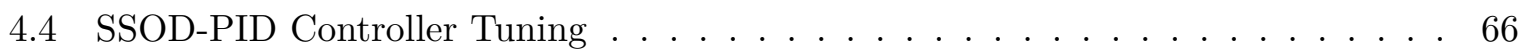

4.4.1 Preliminary issues on dimensionless analysis . . . . . . . . . . . . 67

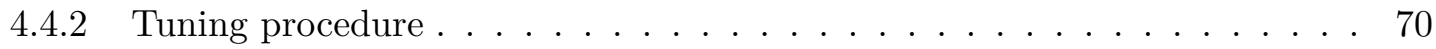

4.4.3 Calculating the dimensionless controllers . . . . . . . . . . . . . . . . 71

4.4 .4 Software tool for tuning . . . . . . . . . . . . . . . . . . 72

4.4 .5 Tuning table . . . . . . . . . . . . . . . . 75

4.5 Effect of the FOPTD approximation $\ldots \ldots \ldots \ldots \ldots$

4.6 Multi-level oscillations . . . . . . . . . . . . . . . . . . . . . . . . 81

4.7 Tsypkin's method approach adapted for RQH sampling $\ldots \ldots \ldots \ldots$. . . . . 84

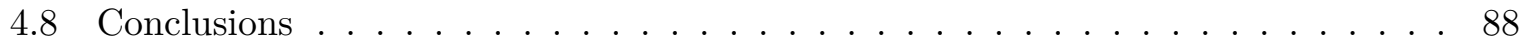

5 Characterization of limit cycle oscillations induced by Fixed Threshold Samplers $\quad 89$

5.1 Introduction . . . . . . . . . . . . . . . . . . . . . 89

5.2 Problem statement . . . . . . . . . . . . . . . . . . . . . 90

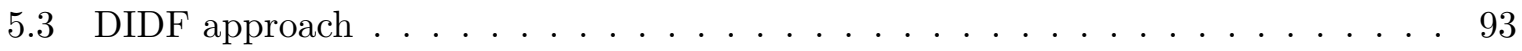

5.3.1 Characteristics of the dynamic part of the DIDF . . . . . . . . . 95

5.4 Oscillation conditions study . . . . . . . . . . . . . . . . . . . . . 99

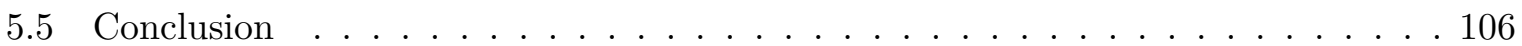


6 Sampled SSOD analysis with DF method 109

6.1 Introduction . . . . . . . . . . . . . . . . . . . . . . . . . 109

6.2 Problem statement . . . . . . . . . . . . . . . . 111

6.3 Sampled Describing Function approach . . . . . . . . . . . . . . . . . . . 113

6.4 Stability analysis . . . . . . . . . . . . . . . 117

6.4 .1 Robustness measure . . . . . . . . . . . . . . . . . . . 121

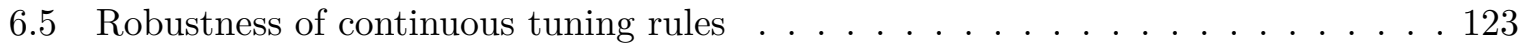

6.5.1 Effect of sampling period on the performance . . . . . . . . . . . . . 127

6.5.2 Influence of model uncertainties on $D_{c h} \ldots \ldots \ldots$. . . . . . . . 129

6.5 .3 Multi-leveled oscillations . . . . . . . . . . . . . . . . . 130

6.6 Conclusions . . . . . . . . . . . . . . . . . . . . . . . . . 130

7 Implementation and experimental evaluation of SSOD and RQH sampling $\begin{array}{ll}\text { strategies for EBC } & 133\end{array}$

7.1 Introduction . . . . . . . . . . . . . . . . . . 133

7.2 Principles of IEC 61499 programming standard . . . . . . . . . . . . 135

7.3 Development of RQH FB in IEC $61499 \ldots \ldots \ldots \ldots$

7.4 Study framework . . . . . . . . . . . . . . . . . . 137

7.5 Experimental validation of EBC controllers . . . . . . . . . . . . . . . 141

7.5 .1 DF method analysis . . . . . . . . . . . . . . . . . 142

7.5.2 Experimental results . . . . . . . . . . . . . . . . . . . . . 143

7.5.3 Tsypkin's method analysis . . . . . . . . . . . . . . . . . . 145

7.5.4 Control period influence . . . . . . . . . . . . . . . . . . 148

7.5 .5 Summary . . . . . . . . . . . . . . . . . . 150

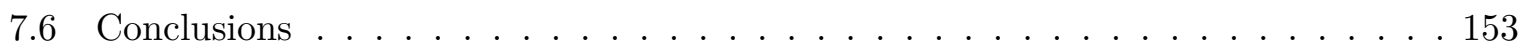

8 Summary and future research $\quad 155$

8.1 Summary . . . . . . . . . . . . . . . . . . . 155

8.2 Future research . . . . . . . . . . . . . . . . . . . . . 158

A Temporal response induced by a multileveled sampler $\quad 159$ 
$\begin{array}{ll}\text { B DF related calculations } & 161\end{array}$

B.1 RQH calculation . . . . . . . . . . . . . . . . . . . . . 161

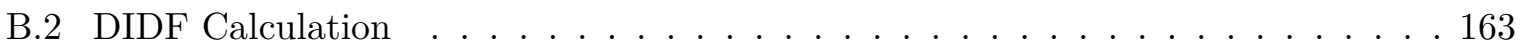

B.3 DIDF maintenance of the bias . . . . . . . . . . . . . . . . . . 164

B.4 Sampled DF calculation . . . . . . . . . . . . . . 165

$\begin{array}{lr}\text { C Tuning methods } & 169\end{array}$

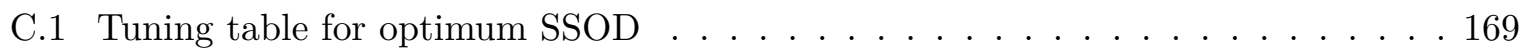

C.2 Flowchart for RQH tuning procedure . . . . . . . . . . . . . . . 169

$\begin{array}{ll}\text { D Supplementary calculus } & 173\end{array}$

D.1 Classical specification dimensionless calculations f . . . . . . . . . . . 173

D.2 IE validity in presence of steady-state error . . . . . . . . . . . . . 174

$\begin{array}{ll}\text { E IEC } 61499 \text { Device Models } & 177\end{array}$

$\begin{array}{ll}\text { Bibliography } & 179\end{array}$ 


\section{List of Figures}

2.1 Control loop scheme for event-based PID controllers proposed in [10]. . . . . . . 10

2.2 Input-output relation of SSOD (left) and RQ (right) sampling strategies. . . . . . 11

2.3 Input-output relation of the proposed sampling strategy with a hysteresis $h$. . . 12

2.4 Influence of $h / \delta$ on the $n_{e v}$ for different values of $\rho \ldots \ldots \ldots \ldots$

2.5 Sampled signal (red) resulting from a sinusoidal signal with and without noise (black) with the generated events (blue) using SSOD, RQ and a RQH sampler with $h / \delta=2 / 3 \ldots \ldots \ldots \ldots \ldots \ldots \ldots \ldots \ldots \ldots$

2.6 Block scheme equivalent to the one presented in Figure 2.1. . . . . . . . . 15

2.7 Shapes of $-1 / \mathcal{N}$ for RQH samplers with different values of $h / \delta \ldots \ldots \ldots \ldots$

2.8 Gain and phase margins to the non-linearity $\left(\gamma_{h / \delta}, \Phi_{h / \delta}\right)$ for a given open-loop transfer function $G_{o l}$ in Nyquist and Nichols diagrams. . . . . . . . . . . . 18

2.9 Nichols plots of the presented batch of processes with the specified controllers and the traces of $-1 / \mathcal{N}$ for $m=1$ with different values of $h / \delta \ldots \ldots \ldots 21$

2.10 Nyquist plots of the presented batch of processes with the specified controllers and the traces of $-1 / \mathcal{N}$ for $m=1$ with different values of $h / \delta \ldots \ldots 21$

$2.11 \gamma_{h / \delta_{d B}}$ and $\Phi_{h / \delta}$ for several values of $h / \delta$ (solid colored lines) and classical gain and phase margins (dashed magenta line) with Ziegler-Nichols tuning method. . 22

$2.12 \gamma_{h / \delta_{d B}}$ and $\Phi_{h / \delta}$ for several values of $h / \delta$ (solid colored lines) and classical gain and phase margins (dashed magenta line) with Cohen-Coon tuning method. . . . 22

$2.13 \gamma_{h / \delta_{d B}}$ and $\Phi_{h / \delta}$ for several values of $h / \delta$ (solid colored lines) and classical gain and phase margins (dashed magenta line) with AMIGO tuning method. . . . . . 23

2.14 Nyquist diagram of $G_{o l}(j \omega)$ and the inverse negative of the DF corresponding to several samplers. . . . . . . . . . . . . . . . . . . . . . 24

2.15 Controlled output $y$ and control action $u$ for the system containing a SSOD sampler, which results in a number of events generated $n_{e v}=31$, marked in orange. 25 
2.16 Controlled output $y$ and control action $u$ for the system containing a RQH sampler, which results in a number of events generated $n_{e v}=22$, marked in orange. . 25

2.17 Controlled output $y$ and control action $u$ of a system with a RQH sampler with $h / \delta=0.4286$ in the loop, which leads to an oscillatory response. . . . . . . . . 26

2.18 Nyquist diagram of $G_{o l}$ with the inverse negative of the DF of its respective sampler. ............................. 27

2.19 Controlled output $y$ and control action $u$ of a system with a RQH sampler with $h / \delta=0.25$ in the loop, which avoids limit cycle oscillations. . . . . . . . . . . . 27

2.20 Modeled system with the best and worst case scenarios due to uncertainty with their respective robustness margins to the non-linearity. . . . . . . . . . . . . 28

3.1 Relationship between input $x$ and output $\bar{x}$ for the RQH sampling strategy. . . . 35

3.2 Shapes of $-1 / \mathcal{N}$ for RQH samplers with different values of $h / \delta$ in Nyquist and Nichols diagrams with the gain and phase margins to the non-linearity $\gamma_{h / \delta}$ and $\Phi_{h / \delta}$. . . . . . . . . . . . . . . . . . . . . . . . . 36

3.3 Graphical interpretation of the detuning procedure to fulfill the $\Phi_{h / \delta_{r}}$ margin. . . 41

3.4 Graphical representation of the gain and phase margins to the non-linearity $\left(\gamma_{h / \delta}, \Phi_{h / \delta}\right)$ in Nyquist (left) and Nichols (right) diagrams for the PI controller obtained in Example 5.

3.5 Temporal response of the controlled output and control action to unitary step-like changes in reference and disturbance of the example system with the proposed controller and sampler. . . . . . . . . . . . . . . . . . . . 44

3.6 Temporal response of the controlled output and control action to unitary steplike changes in reference and disturbance of the example system considering a limitation on the actuator $\delta_{u}=0.05$. . . . . . . . . . . . . . . . . . . . . 45

3.7 Gain and phase margins to the non-linearity $\left(\gamma_{h / \delta}, \Phi_{h / \delta}\right)$ obtained in Example 6 for SSOD sampler (left) and sampler with ratio $h / \delta=1 / 20$ (right). . . . . . . . 45

3.8 Nyquist plot of $G_{o l}$ with the obtained controllers and the inverse negative of the DF for both scenarios. . . . . . . . . . . . . . . . . . . . . 46

3.9 Temporal response of the measured output of the process with the controllers obtained in example 7 (blue: RQH and red: SSOD), the events generated and their respective control actions to unitary step changes in the reference (at $t=0 s$ ) and disturbance (at $t=35 s$ ) inputs. . . . . . . . . . . . . . . . . . . 47

3.10 Inverse negative of the sampler's DF in this example and the $G_{o l}(j \omega)$ of the four studied controllers and process. . . . . . . . . . . . . . . . . . . 49

3.11 Temporal response of the measured output of the process with different controllers, the events generated and their respective control action to unitary step changes in the reference (at $t=0 s$ ) and disturbance (at $t=60 s$ ) inputs. (Orange: proposed controller. Green: AMIGO. Red:One-Third. Blue: Ziegler-Nichols) . . . 
3.12 Sinusoidal signal and its sampled output with a sampler with a given ratio $h / \delta$. . 51

3.13 Harmonic analysis of the validity of the proposed margins for $G_{o l}(s)$ and the sampler with ratio $h / \delta=2 / 3 \ldots \ldots \ldots \ldots \ldots \ldots \ldots$

3.14 Harmonic analysis of the validity of the proposed margins for a given system and sampler. . . . . . . . . . . . . . . . . . 55

4.1 Networked control system with SSOD sampling strategy. SSOD- $C(s)$ architecture. 60

4.2 Non-linear equivalent system to the control system with SSOD sampling strategy in Figure 4.1. . . . . . . . . . . . . . . . . . . . . . . . 6 61

4.3 Plots of the open-loop transfer functions obtained with the PID parameters in Table 4.1 (colored lines) and $-1 / \mathcal{N}$ (black lines) in the Nyquist diagram. . . . . 62

4.4 Steady-state oscillations of the system with $G(s)$ given by equation (4.2) with the PID parameters in Table $4.1 \ldots \ldots \ldots \ldots$. . . . . . . . . . 63

4.5 General form of an oscillation, which define the oscillation conditions in Tsypkin's method . . . . . . . . . . . . . . . . . . . 64 64

4.6 Nyquist diagram of $G_{o l}(\omega)$ and the Tsypkin band for a non-oscillating system $\left(M_{T}=0.23\right)$.

4.7 Nyquist diagram of $G_{o l}(\omega)$ and the Tsypkin band for a system with steady-state

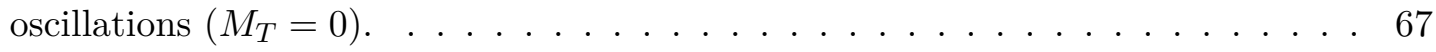

$4.8 M_{T}$ for FOPTD tuned with Ziegler-Nichols (ZN), Cohen-Coon (CC) and AMIGO tuning methods. . . . . . . . . . . . . . . . . . . 68

4.9 Summary of the calculus to obtain the dimensionless set of parameters for the controllers. . . . . . . . . . . . . . . . . . . . . 72

4.10 Evolution of $M_{T}$ with $L / \tau$ for different configurations of $\phi_{m}$ and $N \ldots \ldots 73$

4.11 Obtained values of $\overline{I A E}$ for the considered batch for different configurations of

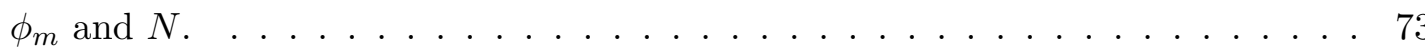

4.12 Obtained values of $\bar{C}(\infty)$ for the considered batch for different configurations of

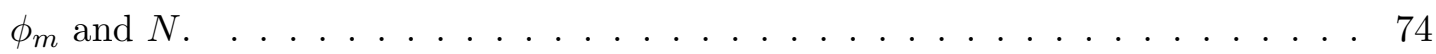

4.13 Summary of the controller design procedure. . . . . . . . . . . . 75

4.14 Software tool process definition window. . . . . . . . . . 76

4.15 Software tool PID design window. . . . . . . . . . . . . 76

4.16 Software tool time response window. . . . . . . . . . . . . . 77

4.17 Software tool PID design window with modified parameters. . . . . . . . . 78

4.18 Software tool time response window with modified parameters. . . . . . . . . . 79 
4.19 Nyquist plot of the open-loop transfer function with all the controllers and their minimum Tsypkin branch. . . . . . . . . . . . . . . . 79

4.20 Disturbance response for the considered system with all the controllers. . . . . . 80

4.21 Control action and events generated by crossing the SSOD levels in black for the considered system with all controllers. . . . . . . . . . . . . . . 81

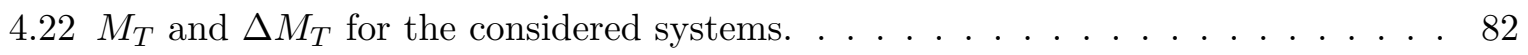

$4.23 R_{1}, R_{2}$ and $R_{3}$ for the batch of models with their respective controller obtained in the previous section. . . . . . . . . . . . . . . 84

4.24 Nyquist plot of the open-loop transfer function with all the controllers and their minimum Tsypkin branch. . . . . . . . . . . . . . . . . . 87

4.25 Temporal response of the error signal $e(t)$ and sampled error signal $\bar{e}(t)$ against disturbance step change of magnitude 2.5 for the four samplers. (Same disposition and colors as in Figure 4.24$) \ldots \ldots \ldots$. . . . . . . . . . . . 87

5.1 On the left image, sinusoidal oscillation sampled with a given FTS. On the right: input-output relationship of the chosen FTS (parameters: $\Delta \delta / \delta=1 / 2, \Delta \epsilon / \epsilon=1 / 4$,

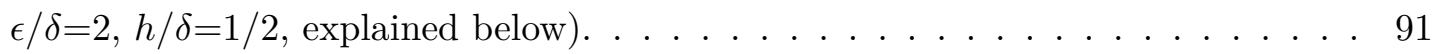

5.2 Relationship between input and output of a generic FTS. . . . . . . . . . . . 93

5.3 Generic loop structure that allows the DF technique application. . . . . . . . . . 94

$5.4 \mathcal{N}_{A}$ of a sampler with two series of traces regarding the levels crossed in each direction (Parameters used: $\Delta \epsilon / \epsilon=\Delta \delta / \delta=1 / 3, \epsilon=\delta=h$ ) . . . . . . . . . . 96

5.5 Radial variance of $-1 / \mathcal{N}_{A}$ with the ratio $\epsilon / \delta$ (Parameters used: $\Delta \epsilon / \epsilon=1 / 4, \Delta \delta / \delta=1 / 3$

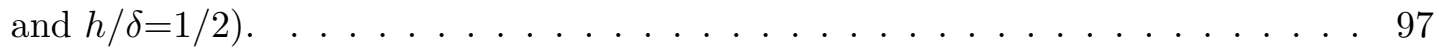

5.6 Effect of the hysteresis on $-1 / \mathcal{N}_{A}$ (Parameters used: $\Delta \epsilon / \epsilon=1 / 5, \Delta \delta / \delta=1 / 2$ and

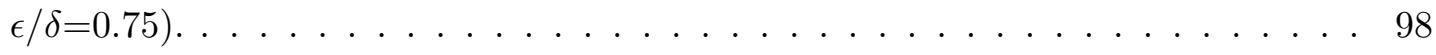

5.7 Asymmetry compensation with the bias $B$ and its effect on $-1 / \mathcal{N}_{A}$ (Parameters used: $\Delta \epsilon / \epsilon=1 / 2, \Delta \delta / \delta=1 / 2$ and $\epsilon=\delta=h) \ldots \ldots \ldots \ldots$. . . . . . . 99

5.8 Input-Output relationship of the sampler used in Example 18 (Parameters: $\Delta \epsilon / \epsilon=\Delta \delta / \delta=0$,

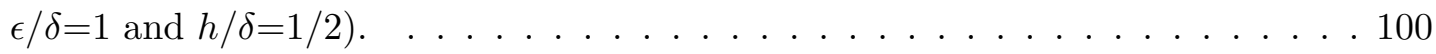

5.9 Graphical representation of the oscillation conditions for Scenario 1. . . . . . . 101

5.10 Temporal response of the error $x(t)$ and sampled error $\bar{x}(t)$ signals under the conditions of the Scenario 1. . . . . . . . . . . . . . . . . 102

5.11 Graphical representation of the oscillation conditions for Scenario 2. . . . . . . 102

5.12 Temporal response of the error $x(t)$ and sampled error $\bar{x}(t)$ signals under the conditions of the Scenario 2 . . . . . . . . . . . . . . 103

5.13 Graphical representation of the oscillation conditions for Scenario 3. . . . . . . 103 
5.14 Temporal response of the error $x(t)$ and sampled error $\bar{x}(t)$ signals under the conditions of the Scenario 3 . . . . . . . . . . . . . . . . . . . . 104

5.15 Graphical representation of the oscillation conditions for Scenario 4. . . . . . . . 104

5.16 Temporal response of the error $x(t)$ and sampled error $\bar{x}(t)$ signals under the conditions of the Scenario 4 . . . . . . . . . . . . . . . 105

5.17 Graphical representation of the oscillation conditions for Scenario 5. . . . . . . 105

5.18 Temporal response of the error $x(t)$ and sampled error $\bar{x}(t)$ signals under the conditions of the Scenario 5 . . . . . . . . . . . . . . . 106

6.1 Sampled system configuration with SSOD non-linearity. . . . . . . . . . . . . 112

6.2 Sine wave in black, quantified by the SSOD_ZOH block in dashed blue, and sampled according to a given sampling period in red (sampling time indicated with red arrows). . . . . . . . . . . . . . . . . . . . . 113

6.3 Sampled DF for different values of $r$, all of them considering $T_{s}=1 \ldots 115$

6.4 Sine wave quantified by a SSOD and sampled resulting in two different oscillation modes. . . . . . . . . . . . . . . . . . . . . . 115

6.5 Detail of the branch $m=1$ of the Sampled DF with $T_{s}=1$ and $r=50$ with a mode region highlighted. . . . . . . . . . . . . . . . . 116

6.6 Graphical representation of the evaluated range of frequencies. . . . . . . . . . 119

$6.7 G_{o l}(j \omega)$ and $-1 / \mathcal{N}$ for the continuous case. The presented PI avoids limit cycle oscillations in this case. . . . . . . . . . . . . . . . 120

6.8 Sampled DF for $m=1$ with $T_{s}=0.5$ considering different values of $r \ldots \ldots 121$

6.9 Controlled output and control action temporal response to a unitary step change in the reference and disturbance inputs with the discrete (green) and continuous (red) controller. The limit cycle predicted from the Sampled DF analysis can be observed. . . . . . . . . . . . . . . . . . . . . 122

6.10 Representation of the distances from the evaluated points to their respective convex hull and detail of $D_{c h}$ for the studied system. . . . . . . . . . . . . 123

6.11 Controlled output and control action temporal response to a unitary step change in the reference and disturbance inputs with the discrete (green) and continuous (red) controller. The absence of limit cycle oscillations predicted by the Sampled DF analysis can be observed. . . . . . . . . . . . . . . . . . 124

$6.12 D_{c h}$ for the batch of processes with different controllers considering $T_{s}=T_{r} / 10$. 125

6.13 Some convex hull plots for process 62 . The convex hull of $-1 / \mathcal{N}^{\prime}(3)$ overtakes the rest, defining $D_{c h} \ldots \ldots \ldots \ldots \ldots \ldots$

$6.14 D_{c h}$ for the batch of processes with different controllers considering $T_{s}=T_{r} / 20.126$ 
6.15 Variation of $D_{c h}$ produced by an increase in the sampling frequency. . . . . . . . 127

6.16 IAE* for the batch of processes with different controllers (circles: $T_{s}=T_{r} / 3$,

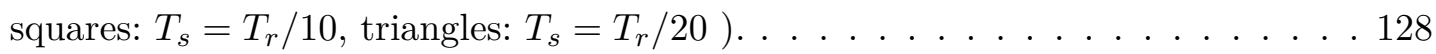

$6.17 D_{c h}$ for the batch of processes with different controllers considering $T_{s}=T_{r} / 3$. $\quad 129$

6.18 Difference between the robustness against oscillations of type $m=2$ and of type

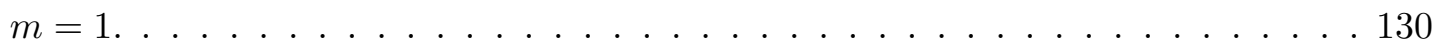

7.1 Interface of the FB RQH_quant. . . . . . . . . . . . . . . . 137

7.2 Execution Control Chart describing the internal behavior of the FB RQH_quant. 137

7.3 Example of the functioning of the RQH_quant FB. Signal to sample in solid black, theoretic result in solid blue, output of the FB in solid red, REQ events in red arrows and EVT events in violet arrows. . . . . . . . . . . . . 138

7.4 System with two connected tanks. Schematic representation and real system. . . 139

7.5 Detail of the actuator (left) and sensor (right) units. . . . . . . . . . . . 139

7.6 Actuator and sensor units. The connection between elements is supported via Ethernet. . . . . . . . . . . . . . . . . . . . . . . 140

7.7 Devices of the study framework and their connection in the System Model. . . . 140

7.8 General layout of the Application Model of the study framework. . . . . . . . . . 141

7.9 Frequency response of the open-loop transfer function and the DF traces of the corresponding sampler. . . . . . . . . . . . . . . . . . . . 143

7.10 Temporal response of the system with different controllers under SSOD sampling. (Blue: Ziegler-Nichols, Red: AMIGO, Light green: SLR, Dark green: SHR, Orange: Tsypkin) . . . . . . . . . . . . . . . . . . . . . . 144

7.11 Temporal response of the system with different controllers under RQH sampling. (Blue: Ziegler-Nichols, Red: AMIGO, Light green: SLR, Dark green: SHR, Or-

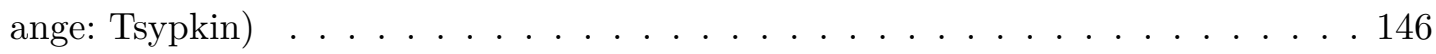

7.12 Critical Tsypkin Branch and $M_{T}$ for those cases where the DF is safely applicable. (Blue: Ziegler-Nichols, Light green: SLR, Dark green: SHR, Orange: Tsypkin) . 147

7.13 Critical Tsypkin Branch and $M_{T}$ for AMIGO controller. Whole representation and detail. . . . . . . . . . . . . . . . . . . . . . . . 148

7.14 Critical Tsypkin Branch and $M_{T}$ for RQH-PI loops (Red: AMIGO, Dark green: SHR, Orange: Tsypkin, Blue: Ziegler-Nichols, Light green: SLR) . . . . . . . . . . 149

7.15 Sampled DF analysis for SHR controller. . . . . . . . . . . . . . . . . 150

7.16 Step response of the studied system with different control periods and respective control action. $T_{s}=200 \mathrm{~ms}$ in dark green, $T_{s}=14 \mathrm{~s}$ in red and $T_{s}=28 \mathrm{~s}$ in blue. 151 
7.17 Response against disturbance of the studied system with different control periods and respective control action. $T_{s}=200 \mathrm{~ms}$ in dark green, $T_{s}=14 \mathrm{~s}$ in red and

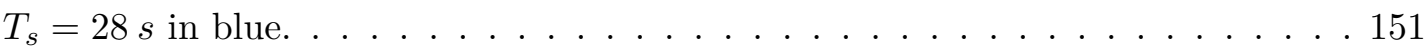

C.1 Flowchart that summarizes the tuning procedure. . . . . . . . . . . . 171

E.1 Device Model of the computer's Interface. . . . . . . . . . . . . . . . . . 177

E.2 Device Model of the actuator unit. . . . . . . . . . . . . . . . 178

E.3 Device Model of the sensor unit. . . . . . . . . . . . . . . . . . . . . 178 



\section{List of Tables}

3.1 Controllers and performance indexes for each controller and sampler under restrictions $S_{1}$ and $S_{2} \ldots \ldots \ldots \ldots \ldots \ldots \ldots \ldots \ldots$

3.2 Obtained controller and performance parameters for each tuning method. . . . . 48

4.1 PID Parameters with different tuning rules for the system presented in equation

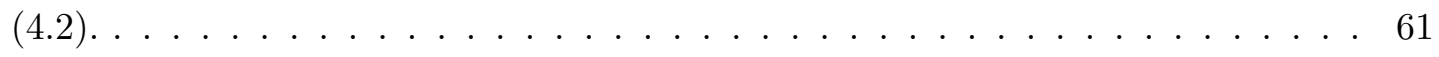

4.2 Summary of characteristics for the studied systems. . . . . . . . . . . . 70

5.1 Summary of samplers and their parameter configuration. . . . . . . . . . . . 92

7.1 Parameters for the different PI controllers for the two tank system. . . . . . . . . 142

7.2 Summary of robustness margins provided by the controllers under study. . . . . . 152

7.3 Summary of detected oscillation and validity of the DF approach. . . . . . . . 152

B.1 Expanded requirements for any combination of process and controller for the maintenance of the bias under step-like changes in reference and disturbance inputs. $L_{1}(s)$ and $L_{2}(s)$ have neither poles nor zeros at the origin and it is con-

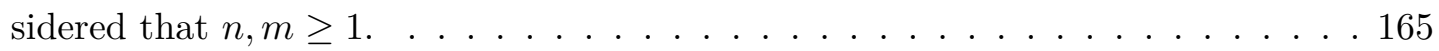

C.1 Tuning table for $M_{T_{r}}>$ 0.2. $(*)$ : Take the parameters for the precedent case of

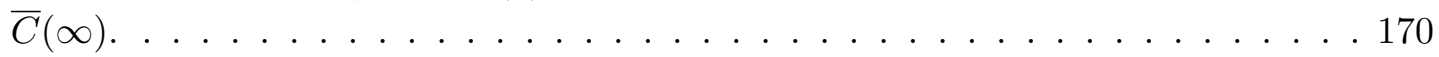





\section{Notation}

\section{Abbreviations}

\begin{tabular}{ll} 
EBC & Event-Based Control \\
PID & Proportional-Integral-Derivative controller \\
SOD & Send-On-Delta \\
SSOD & Symmetric-Send-On-Delta \\
FOPTD & First Order Plus Time Delay \\
RQ & Regular Quantization \\
DF & Describing Function \\
PLC & Programmable Logic Computer \\
RQH & Regular Quantization with Hysteresis \\
DIDF & Dual Input Describing Function \\
FTS & Fixed Threshold Sampler \\
EBPID & Event-Based PID \\
ZOH & Zero Order Hold \\
FO & Fractional Order \\
IE & Integral Error \\
IAE & Integral of the Absolute value of the Error \\
IE & Integral of the sampled Error \\
IAE & Integral of the Absolute value of the sampled Error \\
ADC & Analog to Digital Converter \\
FB & Function Block \\
ECC & Execution Control Chart \\
PWM & Pulse Width Modulated signal \\
\hline
\end{tabular}




\section{Symbols}

\begin{tabular}{|c|c|}
\hline $\mathbf{e}_{\mathrm{ss}}$ & steady-state error \\
\hline$\delta_{\mathbf{u}}$ & control action change due to a variation $\delta$ \\
\hline $\mathrm{G}_{\mathrm{ol}}$ & open-loop transfer function \\
\hline $\mathcal{N}, \mathcal{N}_{\mathbf{A}}$ & describing function for sustained oscillations \\
\hline $\mathcal{N}_{\mathbf{B}}$ & describing function for sustained bias \\
\hline $\mathcal{N}^{\prime}$ & sampling time normalized describing function \\
\hline$\Re\{\cdot\}$ & real part of complex number \\
\hline$\Im\{\cdot\}$ & imaginary part of complex number \\
\hline$\gamma_{\mathbf{h} / \delta}$ & gain margin to non-linearity \\
\hline $\mathbf{\Phi}_{\mathrm{h} / \delta}$ & phase margin to non-linearity \\
\hline$|\cdot|$ & modulus of complex number \\
\hline $\arg \{\cdot\}$ & phase of complex number \\
\hline$\gamma_{\mathrm{cg}}$ & classical gain margin \\
\hline$\Phi_{\mathrm{cp}}$ & classical phase margin \\
\hline $\mathbf{t}_{\mathrm{s}}$ & settling time \\
\hline $\mathbf{M}_{\mathbf{T}}$ & Tsypkin margin \\
\hline $\mathbf{B}_{\mathbf{T}}\left(\omega_{\mathbf{o}}\right)$ & Tsypkin branch corresponding to frequency $\omega_{o}$ \\
\hline$\overline{\mathbf{s}}$ & dimensionless Laplace variable \\
\hline$\overline{\mathbf{K}_{\mathrm{p}}}$ & dimensionless controller gain \\
\hline$\overline{\mathbf{T}_{\mathbf{i}}}$ & dimensionless integral time \\
\hline$\overline{\mathbf{T}_{\mathrm{d}}}$ & dimensionless derivative time \\
\hline$\overline{\mathbf{G}}(\overline{\mathbf{s}})$ & dimensionless transfer function \\
\hline $\mathbf{C}(\infty)$ & noise amplification \\
\hline$\overline{\mathbf{C}}(\infty)$ & dimensionless noise amplification \\
\hline $\mathbf{R}_{\mathrm{m}}$ & Tsypkin robustness against m-leveled oscillations \\
\hline $\mathbf{C}(\mathbf{z})$ & z-transform of controller \\
\hline $\mathbf{C}^{*}(\mathbf{s})$ & starred transform of controller \\
\hline $\mathrm{D}_{\mathrm{ch}}$ & distance to convex hull \\
\hline
\end{tabular}




\section{Introduction}

\subsection{Background}

In the last decades Event-Based Control (EBC) of continuous systems has been increasingly applied to industrial applications. Whereas the time-triggered controllers, traditionally used in industry, perform their control algorithms with a fixed period, the event-based controllers execute their control algorithms only after the reception of asynchronous events which indicate significant changes in the state of the system. A typical example of this type of event reception policy could be ruled by a sampler which sends an event whenever the error signal (difference between reference or set-point and the controlled output) crosses certain thresholds.

EBC pursues two paramount objectives:

1. Reducing the amount of data necessary to perform the closed-loop control.

2. Diminishing the average computational cost that the control algorithms require.

Due to these characteristics, EBC algorithms have been mainly applied in distributed control systems to reduce the data flow through industrial communication networks, for instance, between sensor nodes, controllers and actuators. Several control strategies have been adapted to EBC, including the PID controller, widely used in the industry [62], which counts with several versions and tuning methods suitable for EBC.

Among the first contributions to the development of Event-Based PID controllers Årzén's controller [3] was introduced. This event-based controller provided a reduction in the usage of the CPU in EBC systems controlled by a computer without affecting significantly the behavior of the control loop. Some of the most important aspects about EBC were made in that contribution, namely, the error produced in the calculation of integral and derivative terms when the time between samples increases. Some posterior works have been conducted to resolve the problems unveiled by Årzén, mainly related to the error in the calculation of the integral term. In that sense, the contributions made by Durand [22, 23] and Vasyutynskyy [76, 77] must be highlighted. 
Regarding the event generation strategy implemented in EBC, the most used, specially when a PID controller is used, are those in which new data are sent when the sampled signal crosses certain thresholds of value $\delta$. That event generation policy is known as Send-On-Delta (SOD) and its efficacy in terms of control loop performance and in the reduction of the communication data flow has been contrasted in $[19,50]$.

A variant of the SOD sampling technique applied to PI control was first proposed in [10]. The variation resides in, unlike in the SOD sampling, the signal, which is quantified in multiples of the minimum threshold $\delta$, in that way, the relationship between the input and output of the event generator is symmetric with regard to its origin. This strategy is known as SymmetricSend-On-Delta (SSOD). Some preliminary results in the study of PI controllers with SSOD sampling have been presented in [10, 17]. In [11] a tuning procedure for PI controllers under SSOD sampling for first order plus time delay (FOPTD) systems has been proposed, therefore, the obtained results are limited to this kind of models.

More recent results have been presented in $[60,59,56,58]$. In [60] the authors present a tuning rule for PID controllers based on SSOD sampling. This tuning rule is based on the usage of phase and gain margins, which are parameters widely used in the classical approach to the controller tuning problem, which facilitates its application. Likewise in [56] the tuning method is improved and it also guarantees an optimal behavior of the controller with regard to the perturbation response, minimizing the integral error of this response, while guaranteeing robustness against limit cycle oscillations induced by the SSOD sampler.

On the other hand, in [59] the effect of introducing different sampling schemes in the PID control loop was studied. Particularly, the sampling strategies under study where the SSOD and the regular quantification (RQ). Using as analysis tool the Describing Function (DF) technique, the conditions that a system must fulfill to avoid limit cycles induced by these types of sampling techniques were obtained. These conditions are given in both cases in terms of gain and phase margins. In [58] a comparative study of the robustness attained with RQ and SSOD was performed, in addition, the advantages and drawbacks of each sampling strategy were highlighted.

Other approximations to Event-Based PID controllers are also possible, for instance, in [55] a controller with adaptive thresholds was proposed. This flexible sampling strategy attained a balance between the number of generated events and the magnitude of the perturbations.

\subsubsection{Implementation of EBC in the standard IEC 61499}

In industrial automation there exist several approaches to program an application, however, most of them are included in the precepts presented in the standard IEC 61131 [32]. Nevertheless, this standard presents some drawbacks, being the most important the difficulty in distributing applications through several devices. To solve this problem, the standard IEC 61499 [80] was created, whose goal is to facilitate the design and implementation of distributed and reconfigurable applications. This last standard presents an interesting feature with regard to 
the former one, which is its execution model. While IEC 61131 is based on the periodic execution of the application algorithms, the IEC 61499 is based on an event-driven execution model, substantiated with the management and treatment of events.

Considering that the amount of research concerning the usage of IEC 61499 in discrete Event-Based Control applications is considerable, it cannot be equally said about its usage for continuous control systems despite its importance in the industrial environment.

With regard to this research line, it can be found [71], which was one of the firsts works to highlight the importance of giving to the IEC 61499 a deterministic behavior in terms of execution times to build upon consistent control applications. In [21], an experimental study about the applicability of the standard to the control of a robotic arm using a PID controller was performed, showing its viability in this kind of applications. Other works like [29], emphasize on the considerations that must be taken into account when implementing a controller for a continuous system in IEC 61499. In [16], the IEC 61499 standard was used to implement a distributed control of a flexible manufacturing system. Regarding the management of events, in [51] a study was presented in which the importance of a proper implementation of the event management for the control of continuous systems was highlighted.

Despite these efforts in this research line, most of the works involving IEC 61499 for continuous control consider uniquely time-triggered controllers. By using this kind of control algorithms, the event-driven execution model characteristic of IEC 61499 programming is missed. This feature of the standard suggests that EBC could be a more suitable algorithm for the control of continuous systems.

\subsection{Challenging problems}

A lot of interesting results have been obtained in the research field of Event-Based PID controllers recently. However, there still exist some important questions to be answered. The main objective of this thesis tries to give an answer to some of these challenging problems.

\section{Development of specific analysis and tuning methods}

In the literature a lot of control schemes in which event-based PID controllers are involved have been proposed, not paying sufficient attention to the tuning methods for designing these controllers. Actually, in most of those works the tuning methods used for designing the controllers belong to continuous control loop schemes or, at best, belong to sampled or hybrid control schemes. The extension of these methods to the real event-based scenario is based on empirical ideas but not on a rigorous study of the stability conditions, robustness margins and performance of the system. In addition, Event-Based Controllers are usually implemented in devices based on microprocessors, like PLC's in industrial environments or cards such as Raspberry, BeagleBone Black, etc. The controllers on these devices are usually implemented as periodic routines, for which there exist specific guidelines to choose the sampling and control periods, which are paramount parameters. However, in the most similar version of this 
implementation, i.e. the periodic triggered event-based control, these guidelines do not apply. Therefore, specific analysis tools to asses the performance and robustness of a given system are required, as well as tuning rules, based on these analysis results, which assure a good performance and robustness of the controlled system.

\section{Study of new sampling schemes}

Traditionally, Event-Based PID controllers have used sampling schemes based on crossing levels, being SOD and some of its variations as the SSOD among the most used. Some works indicate that depending on the application some other sampling strategies could be more beneficial in terms of generated events and robustness for a non detrimental performance of the loop. These new sampling strategies will require the development of some specific analysis methodologies and tuning methods for the controllers that take into account the characteristics of the strategy.

\section{Implementation of Event-Based PID controllers in the standard IEC 61499}

Hitherto, methods for implementing systematically applications of Event-Based Control to the standard IEC 61499 do not exist. Furthermore, the controllers used for regulating continuous processes under this standard are discrete controllers with periodic execution, wasting the opportunity of benefiting from the event-based execution of algorithms that IEC 61499 offers. Hence, it seems that there exist some synergy between Event-Based Control systems and the standard IEC 61499: Event-Based Control will provide algorithms and control methodologies suitable for the distributed control of continuous processes to the standard and the standard will facilitate the implementation, setting, verification and maintenance of the networked control system. Needless to say, the control algorithms will improve the computation time required without affecting significantly the performance of the control loop.

\subsection{Thesis outline}

The aim of this thesis is to provide some techniques and methodologies to study the robustness of loops in which Event-Based PID controllers are involved. Different sampling techniques based on fixed-threshold quantization, which are in charge of generating the events, are studied. Once the robustness has been characterized, several designs methods have been proposed which take into account both the robustness and the control loop performance. Therefore, the results presented in each of the chapters orbit around these issues focusing on different aspects or under distinct approaches.

The thesis develops firstly the theoretical contents corresponding to the analysis and design of controllers taking into account the sampling strategy integrated in the control loop. The performance of the loop sampled with these sampling strategies is assessed using different analysis tools. Chapters 2-6 are dedicated to the study of SSOD and RQH sampling strategies and their effect on the loop performance and robustness under different theoretical frameworks.

The implementation of the studied Event-Based Controllers in the previous theoretical chapters is described in Chapter 7. To that end, the standard IEC 61499 is used.The aim of this 
standard is to develop the software for distributed control systems, so in general, it facilitates the implementations of networked control systems as those studied in this thesis.

Each chapter has been conceived to allow its lecture independently of the others. The contents presented in the thesis derive from material in press or published and, therefore, some repetitions may appear on the preliminaries of each chapter. A more detailed summary of the content of the chapters is now presented.

\section{Chapter 2: RQH sampling strategy analysis with DF method}

In this chapter the benefits of RQH sampling over SSOD are discussed. Because of the differences that $\mathrm{RQH}$ introduces a new robustness analysis is performed, and as a consequence some measures in terms of gain and phase are defined to characterize the robustness.

\section{Chapter 3: Tuning Procedure for PI controllers under RQH sampling}

Thanks to the analysis of RQH sampling strategy, the necessity of having specific controllers that take into account the effect of the non-linearity has been spotted. Therefore, a new tuning procedure which takes into account the sampling strategy in the loop with its specific robustness measures has been proposed.

\section{Chapter 4: Analysis and tuning of SSOD and RQH systems with Tsypkin method}

In this chapter the robustness analysis of systems under a SSOD sampling strategy is studied and a tuning procedure for PID controllers is proposed. The robustness is defined in terms of a measure called Tsypkin margin $\left(M_{T}\right)$, which characterizes the presence or avoidance of limit cycle oscillations. Based on this measure, a tuning procedure is proposed, which provides safe controllers. The analysis is extended for covering the limitations of the current RQH sampling analysis, concretely, in studying systems with low filtering capabilities. The Tsypkin method is also used to provide a theoretical base to study this kind of systems with RQH sampling.

\section{Chapter 5: Characterization of limit cycle oscillations induced by Fixed Thresh- old Samplers}

To conform a structure for implementing an EBC there exist many valid sampling strategies apart from the SSOD and RQH samplers. In this chapter the sampling strategies that provide as output a quantification of the input signal with some fixed threshold are studied. These sampling strategies can be considered as a kind of SOD strategy, but, in addition, it can have any type of asymmetry, therefore, they will be referred to as ASOD for Asymmetric-Send-On-Delta. Due to the asymmetry present in these types of samplers, the method used for the analysis is a variation of the DF known as DIDF.

\section{Chapter 6: Sampled SSOD analysis with DF method}

The aforementioned Event-Based Controllers and sampling strategies are implemented in physical systems. This implementation entails some theoretical differences with regard to the provided studies caused by the apparition of a sampling period. In most implementations, this parameter is of scarce relevance due to the high performance of existing hardware, however, in 
some systems it could play an important role in the robustness. In this chapter, the robustness of discrete implementation of controllers on loops with a SSOD is evaluated.

\section{Chapter 7: Implementation and experimental evaluation of SSOD and RQH sampling strategies for EBC}

This chapter describes the main aspects of IEC 61499 and how they are used to implement SSOD and RQH sampling techniques in a networked control system. The theoretical contents developed theoretically in previous chapters of the thesis are applied to control a real system with SSOD and RQH sampling and prove the validity of the analysis tools when predicting the behavior of a real system.

\section{Chapter 8: Summary and future research}

In this final chapter the conclusions about the current thesis are drawn, and the perspectives about future research problems are also discussed. 


\title{
RQH sampling strategy analysis with DF method
}

\begin{abstract}
In this chapter, a new sampling strategy for networked control systems, called Regular Quantification with Hysteresis (RQH), is proposed. This alternative presents some benefits with respect to symmetric-send-on-delta sampling, which is one of the most used strategies in event-based PID control loops. The behavior of the RQH is defined by two parameters, the signal quantification and hysteresis, whose effect on the overall system performance is studied and guidelines about its choice are given in terms of noise measurement and steady-state error. The limit cycle oscillations that could be induced by this sampling strategy are studied and new robustness measures to avoid them are proposed based on the Describing Function (DF) approach. The suitability of some tuning rules for continuous PI when applied to control systems with a $R Q H$ sampling is evaluated using the proposed measures. The results show that these tuning rules can be applied under certain conditions.
\end{abstract}

\subsection{Introduction}

In recent years, several works have been published about Event-Based Control (EBC) of continuous systems, [38]. EBC allows to economize the data flow through the digital networks on networked control systems, reducing the data drop out in the form of packet losses and decreasing the delays introduced by the communication infrastructures. This is due to the fact that new data are only sent when significant changes are detected on the state of the system, instead of periodically as in the case of time-driven controllers. That is why EBC may be considered as one of the most promising control approaches in networked control systems, whose importance in modern factory automation has been recently recognized in [20]. An updated and extensive study about the main contributions to EBC during the last twenty years can be found in [2]. 
Among the control strategies adapted to the EBC paradigm, the PID algorithm has caught a special attention. It is undeniable that nowadays PID are used in most of the industrial control applications. Some data about the prevalence of PID in industry are given in [48], showing that more than $95 \%$ of the controllers are of this type. In the same line, a survey conducted among the industrial committee members of the International Federation of Automatic Control, published in [62], shows the dominant position of PID algorithms with respect to other advanced control strategies as MPC. Recently, the primordial role of PID in the context of Industry 4.0 has been highlighted in [39], as well as the necessity of introducing new features to adapt this well established control technology to this new paradigm, whose one of the most distinctive signs is the high connectivity between devices through wired and wireless communication networks. This fact, jointly with the dominance of PID in industry mentioned before, has encouraged the development of many researches about the Event-Based PID controllers during the last decades.

To the author's knowledge, one of the first works about Event-Based PID (EBPID) was presented in [3]. In that paper some issues were addressed related with the error in the calculation of the integral and derivative terms when the time between samples increases due to the irregular sampling. This problem was extensively treated some years later in the works of Durand $[22,23]$ and Vasyutynskyy $[77,76]$. Even though the goal in [3] was to use the EBPID to reduce the use of CPU in embedded control systems without significantly affecting the closed-loop performance, the seminal ideas presented in that paper were afterwards extended to the case of EBPID in networked control systems.

As it was early shown [30], the sampling law determines the performance and behavior of sampled control systems. In Event-Based Control systems, the sampling strategies play an important role because they are in charge of generating the events for the execution of the controller's algorithm. Among these strategies, the ones based on the signal quantification have gained more and more relevance because of their ease of implementation. The most representative example is the Send-On-Delta (SOD) sampling, which is based on transmitting the value of a signal only when it crosses levels or thresholds of magnitude $\delta$. The effectiveness of this strategy has been widely tested in terms of control performance and communication reduction, $[19,50]$.

Inspired in SOD, in [10] a sampling strategy known as symmetric-send-on-delta (SSOD) was presented, which is characterized by including a hysteresis of the same value than the thresholds $\delta$ to the sampler. In the last years, several works have been presented about SSOD based PI controllers, concerning both tuning procedures and application cases. In [11], the tuning of SSOD based PI controllers for FOPTD systems was addressed and some rules were designed by minimizing the $1 \%$ settling time of the closed-loop response. An application of SSOD Event-Based Controllers to the inside air temperature control of the greenhouse production process was presented in [49]. In [60] and [56], tuning methods for PI controllers with SSOD sampler have been developed based on new robustness margins to avoid limit cycles that were obtained by applying the Describing Function (DF) technique and connect with the classical concepts of phase and gain margins. In [61], a unified design of a SSOD based PID and Smith predictor for self-regulating and integral processes was investigated. A new system identification 
procedure based on the oscillations induced by SSOD sampling strategy was proposed in [70]. More recently, in [44] a procedure for tuning not only PI but also PID controllers with SSOD sampling for FOPTD systems was proposed. It is based on the definition of a new robustness measure to avoid limit cycle oscillations called the Tsypkin margin, presented in [43], which overcomes the limitations of the describing function approach. All these works reveal the interest in the SSOD based controllers during the last decade.

Alternatively to the SSOD, another sampling strategy is the Regular Quantification (RQ), in which new data are sent whenever the value of the sampled signal is a multiple of the quantification threshold $\delta$. A comparative study between SSOD and the RQ strategy has been presented in [58]. Due to the lack of hysteresis in the RQ sampler, bursts of events can appear due to measurement noise, which is the main disadvantage of RQ with respect to SSOD. On the other hand, in RQ loops the robustness against limit cycle apparition is higher than in SSOD loops for similar loop performances. These results suggest that the intermediate cases between RQ and SSOD sampling strategies could offer a better global behavior because event generation due to noise can be avoided and robustness requirements may be not as restrictive as in the SSOD sampler.

The aforementioned researches in the field of EBPID show that, in general, conventional PID working under event-based sampling strategies hold its good performance-robustness trade-off while drastically reducing the data necessary to perform the control, and consequently the transmission through the digital network in networked systems is also decreased. The results presented in this chapter delve into this approach by proposing new sampling strategies for Event-Based PI that improve the before mentioned drawbacks of the SSOD and RQ samplers.

The sampling law proposed here can be seen as a generalization of the SSOD and RQ. The approach is based on selecting the hysteresis and the quantification threshold independently. This allows disengaging the immunity to noise from the reactivity of the controller to significant changes in the system. It is also proved that the new proposal can significantly reduce the number of generated events with respect to the SSOD strategy. The new sampler is studied in terms of robustness, developing quantitative measures to avoid the appearance of limit cycle oscillations.

Because in PID controllers the derivative action is very sensitivity to measurement noise, the PI control is the dominant form of the PID in use today, [5]. As an example of the prevalence of PI among industrial control loops, the study presented in [72] reveals that more than $94 \%$ of controllers in power plants in Guangdong Province, China, are PI. Using the proposed robustness measures, the suitability of some well known tuning methods used to design continuous PI controllers are evaluated when applied to loops with PI controllers under the proposed sampling strategy. Concretely, the Ziegler-Nichols [79], Cohen-Coon [18] and AMIGO [4] methods are evaluated. These methods can be easily applied in industry because the controllers parameters are straightforward calculated using very simple equations and data collected from step response or relay feedback experiments. Some previous studies developed by the author have proved that AMIGO provides proper tune for this kind of control scheme when Symmetric-Send-On-Delta sampling is used, [44]. Among other results, the study in this chapter demonstrates the validity 


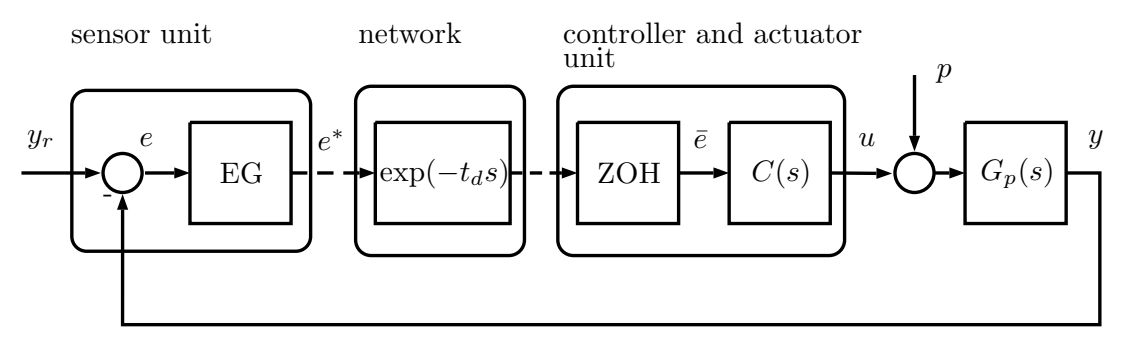

Figure 2.1: Control loop scheme for event-based PID controllers proposed in [10].

of the AMIGO method to avoid the limit cycle oscillations when using PI with the proposed sampling law.

The chapter is organized as follows. In section 2.2 the problem statement is presented. Section 2.3 addresses the main characteristics and advantages of the proposed sampling strategy. Section 2.4 provides a study about the robustness against limit cycles that could be induced by this sampling approach. The study is based on the describing function method and two new robustness margins are defined to avoid limit cycle oscillations. In section 2.5 the aforementioned tuning methods are evaluated according to the proposed robustness margins considering a batch of processes. In section 2.6 several simulation examples are presented. Finally, the conclusions about this work are drawn in section 2.7 .

\subsection{Problem statement}

Consider the networked control system shown in Figure 2.1, where $C(s)$ and $G_{p}(s)$ are the controller and process transfer functions respectively, the EG block represents the event generator, the $\mathrm{ZOH}$ block is a zero-order hold and $\exp \left(-t_{d} s\right)$ models the network's delay. Additionally, $y_{r}$ is the reference signal, $y$ the controlled output and $p$ the disturbance input. It is assumed that the controller is located close to the actuator and that the event generator sends the measured signal $e^{*}$ of the error $e$ through the communication network and the $\mathrm{ZOH}$ block maintains in $\bar{e}$ the last sent value $e^{*}$ until new data arrive. This control scheme was proposed in [10] considering that $C(s)$ is a PI controller and that the EG block is a SSOD sampler, thus, the authors named this architecture SSOD-PI. In a more general way, any controller and event generator can be used, therefore I will refer to this architecture as $E G-C(s)$.

As it has been mentioned before, two of the main sampling strategies used for event generation with fixed thresholds are the SSOD and RQ. The relation between a given input signal $x$ and its respective output $\bar{x}$ for both sampling methods are shown in Figure 2.2. The SSOD is characterized by sending new data whenever the sampled signal changes in a magnitude $\delta$ with respect to the last value sent, whereas the RQ sends new data whenever the signal crosses a value multiple of the quantification magnitude, $\delta$, without any memory of the crossed thresholds.

As it was pointed in [58], with regard to the sampling of noisy signals, the SSOD can avoid the generation of extra events due to noise as long as its amplitude is lower than the hysteresis, 


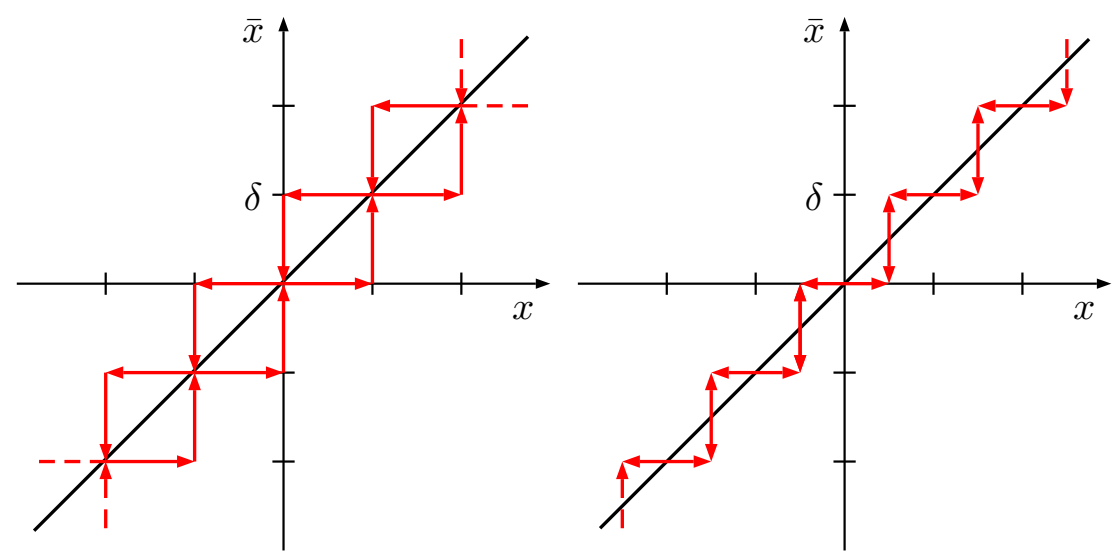

Figure 2.2: Input-output relation of SSOD (left) and RQ (right) sampling strategies.

whose value is equal to the threshold $\delta$. Conversely, the RQ sampler is very sensitive to noise due to the lack of hysteresis, producing a high rate of events near to the crossing levels. On the other hand, regarding the robustness, the SSOD- $C(s)$ configuration propitiates the apparition of limit cycles, mainly due to the hysteresis, and thus, it requires more robust controllers than the RQ- $C(s)$, which admits faster controllers with lower robustness requirements. These negative effects of SSOD and RQ in the control loop should be reduced in order to improve the overall performance of the control system. To this aim, a new sampling law is introduced in the next sections, whose properties are studied by defining new robustness measures to limit cycle.

It must be remarked that for processes with integrator the control system in Figure 2.1 presents an oscillatory response, no matter the sampling strategy used in the block EG. This can be easily seen from the temporal response of the system: as long as the control action is not null, the process will keep integrating it and eventually the commutation thresholds will be reached, inducing the apparition of limit cycles that are unavoidable for this kind of models. A control scheme based on SSOD sampling that prevents this kind of oscillations for integrating processes was proposed in [61]. Therefore, processes with integrator are out of the scope of this chapter.

\subsection{RQH sampling strategy}

As SSOD and RQ are complementary with respect to their strengths and weaknesses, a new sampling strategy that get advantage of both is proposed. This strategy has been named Regular Quantization with Hysteresis (RQH), and its behavior, i.e. the relation between an input signal $x(t)$ and its respective output $\bar{x}(t)$, is defined by equation (2.1) that includes two parameters, the quantization level $\delta>0$ and the hysteresis $h$ that can be freely selected as long as $0 \leq h \leq \delta$. The input-output characteristic of the RQH sampler is presented in Figure 2.3 where it can be easily seen that by fixing $h=0$ or $h=\delta$ the RQ or SSOD samplers are obtained respectively, thus, these sampling strategies are particular cases of RQH. 


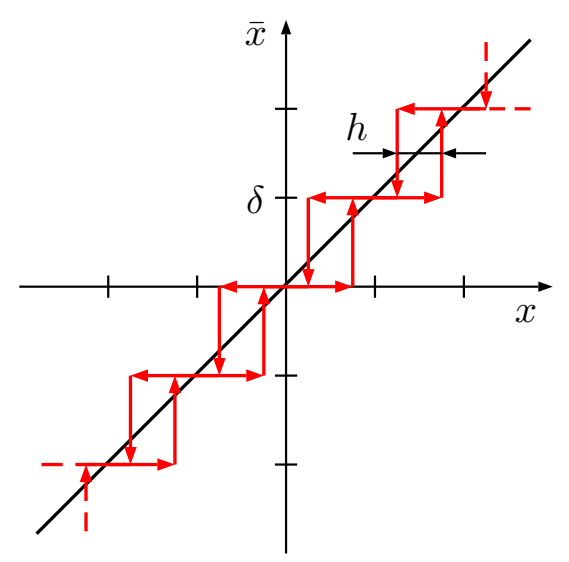

Figure 2.3: Input-output relation of the proposed sampling strategy with a hysteresis $h$.

$$
\bar{x}(t)= \begin{cases}(i+1) \delta & \text { if } x(t) \geq\left(i+\frac{1}{2}+\frac{h}{2 \delta}\right) \delta \text { and } \bar{x}\left(t^{-}\right)=i \delta, i \in \mathbb{Z} \\ (i-1) \delta & \text { if } x(t) \leq\left(i-\frac{1}{2}-\frac{h}{2 \delta}\right) \delta \text { and } \bar{x}\left(t^{-}\right)=i \delta \\ i \delta & \text { if } x(t) \in\left[\left(i-\frac{1}{2}-\frac{h}{2 \delta}\right) \delta,\left(i+\frac{1}{2}+\frac{h}{2 \delta}\right) \delta\right] \text { and } \bar{x}\left(t^{-}\right)=i \delta\end{cases}
$$

For intermediates values of $h$ the proposed method presents characteristics of both SSOD and RQ. In general, the increment of $h / \delta$ reduces the event generation due to the noise for the same measured signal. More concretely, the inclusion of a hysteresis aims to eliminate the burst of events generated by the noise, which are eliminated whenever the amplitude of the noise lays within the hysteresis thresholds. Therefore, $h$ must be selected slightly higher than the peak-to-peak amplitude of the noise.

The RQH sampling, as well as SSOD and RQ sampling, introduces a steady-state error on the system output of Figure 2.1 because it exists a band around $e=0$ in which the controller will receive a sampled error $\bar{e}$ equal to 0 but the value of $e$ is between the commutation thresholds. This band should correspond to the values where there are not significant changes on the process output $y$. Equation (2.2) relates the admissible steady-state error in the controlled output $\left(e_{s s}\right)$ with the parameters of RQH.

$$
e_{s s}=\frac{1}{2}(\delta+h)
$$

If $h$ has been previously defined by considering the measure noise, then $\delta$ can be obtained directly from this expression to fulfill the $e_{s s}$ requirement.

\subsubsection{Event generation}

One of the goals in the design of networked control systems is to reduce the data transmission through the network, which is directly related with the number of events generated by the 


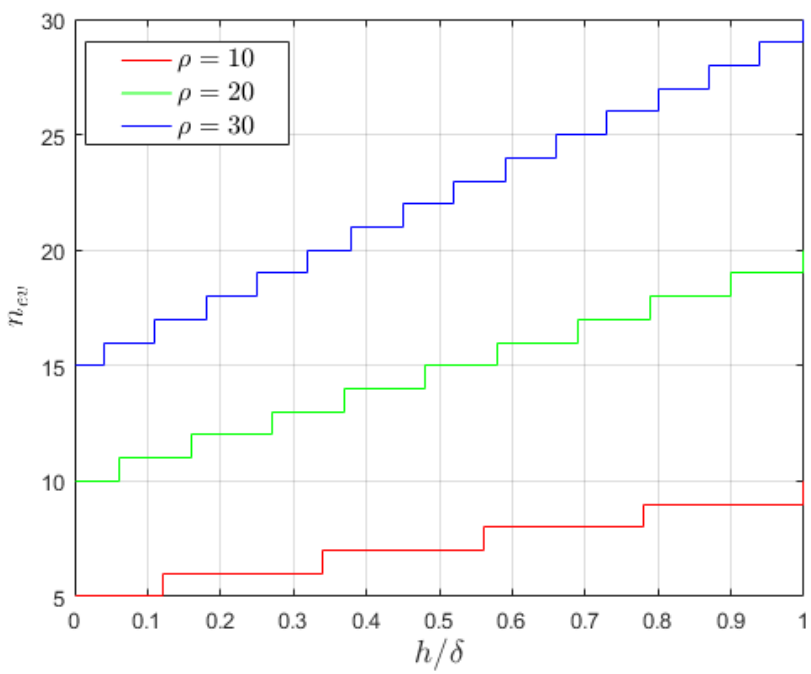

Figure 2.4: Influence of $h / \delta$ on the $n_{e v}$ for different values of $\rho$.

sampler within the event-based control systems. With the proposed RQH sampling strategy the number of events generated for a change of magnitude $C$ in the input signal is:

$$
n_{e v}=\left\lfloor\frac{C-\frac{1}{2}(\delta+h)}{\delta}+1\right\rfloor .
$$

Combining expressions (2.2) and (2.3) the relation between $n_{e v}, h / \delta$, and $C$ can be found:

$$
n_{e v}=\left\lfloor\frac{C-e_{s s}}{2 e_{s s}}\left(1+\frac{h}{\delta}\right)+1\right\rfloor .
$$

If the change $C$ in the input signal is expressed in terms of the admissible steady-state error as $C=e_{s s} \rho$, then:

$$
n_{e v}=\left\lfloor\frac{\rho-1}{2}\left(1+\frac{h}{\delta}\right)+1\right\rfloor
$$

Equation (2.5) describes the number of events generated by a RQH sampler with a given ratio $h / \delta$ when sampling an input signal with a change of magnitude $e_{s s} \rho$. Figure 2.4 shows the relation between $n_{e v}$ and $h / \delta$ for different values of $\rho$. It can be observed that the ratio $h / \delta$ significantly affects the amount of events for the same value of $\rho$. Concretely, the number of events obtained with a $\operatorname{SSOD}(h / \delta=1)$ doubles the events generated by a RQ $(h / \delta=0)$. The $\mathrm{RQH}$ sampler presents intermediate values of $n_{e v}$ between these extreme cases, reducing the amount of events as the $h / \delta$ is reduced. This influence on the number of events with respect to $h / \delta$ is stronger for higher values of $\rho$.

With the aim of shedding further light on the advantages of the RQH sampler with respect to the SSOD and RQ strategies, the following example is introduced. 


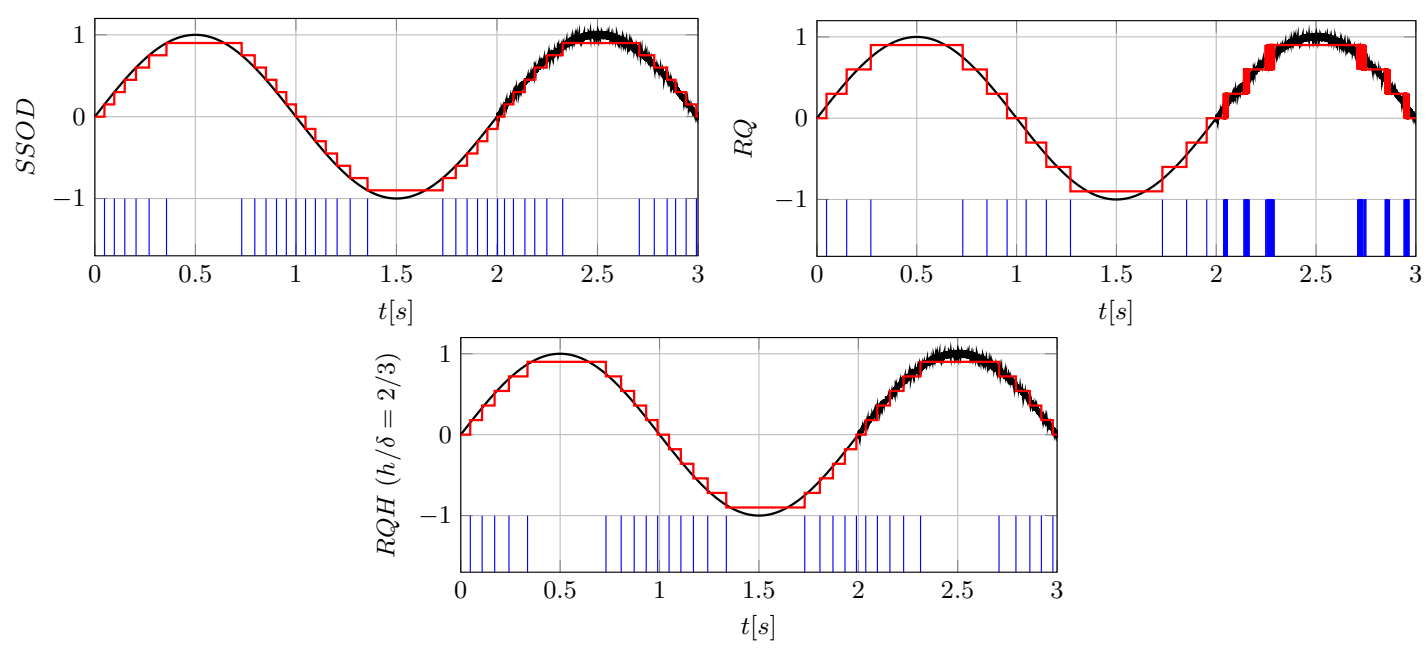

Figure 2.5: Sampled signal (red) resulting from a sinusoidal signal with and without noise (black) with the generated events (blue) using SSOD, RQ and a RQH sampler with $h / \delta=2 / 3$.

Example 1. Consider three samplers (SSOD, RQ and RQH) which should be configured to guarantee a maximum steady-state error $e_{s s}=0.15$. In the case of SSOD and RQH, the samplers should also avoid the event generation due to a noise of 0.1 peak-to-peak amplitude. To fulfill these requirements, the parameter $h$ of the RQH sampler must be selected slightly greater than the amplitude of the noise: $h=0.12$. Then, $\delta$ can be calculated from equation (2.2) to meet the condition on $e_{s s}: \delta=2 e_{s s}-h=0.18$. For the $S S O D$ and $R Q$ sampler, $\delta$ is determined by $e_{s s}$ : for $S S O D \delta=e_{s s}=0.15$ and for $R Q \delta=2 e_{s s}=0.3$. In order to show the behavior of the samplers, consider a sinusoidal input with unitary amplitude. During the first 2 seconds the input to the sampler is a signal without noise, then, the noise is added. The results produced by the samplers are shown in Figure 2.5.

Firstly, it can be noted that the switching thresholds and the quantification produced by each sampler are different. This is due to the $e_{\text {ss }}$ specification, which results in different $\delta$ for each sampler. For the $R Q$ sampler the number of events is significantly lower than for the other two alternatives when a signal without noise is sampled. Nevertheless, for a noisy input, the $R Q$ generates unnecessary events when the input is close to the switching thresholds. The SSOD sampler keeps quantifying the signal without being disturbed by the noise, but, as it has the lowest switching thresholds, if only the sampling of the signal without noise is considered, the number of events generated is the highest one among the three samplers. By using the RQH no bursts of event due to the noise are observed. Moreover, the number of samples is lower than in the case of the SSOD sampler, effectively reducing the data transmission through the network.

Using the RQH sampling strategy has some important implications on the loop performance. As it is shown in the precedent example, the RQH sampler offers a reduction in the data transmission through the network with regard to RQ and SSOD sampling techniques. Nevertheless, regarding the control action bumps due to a change of magnitude $\delta$ in the sampler output $\delta_{u}=K_{p} \delta$, it is clear that by choosing the sampler parameters as in Example 1, these control 


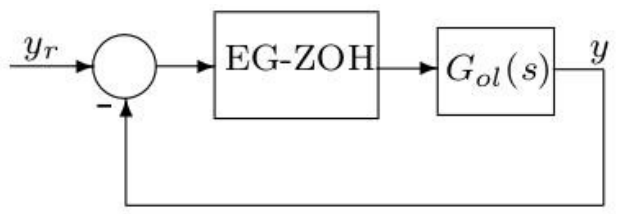

Figure 2.6: Block scheme equivalent to the one presented in Figure 2.1.

action bumps will be greater for the RQH than for the SSOD sampling because the value of $\delta$ is greater for the first one.

Despite this detrimental effect, choosing a RQH sampler has a big influence regarding the robustness requirements to design the controller $C(s)$ in order to avoid limit cycle oscillations induced by the sampler. This issue is studied in the next section.

\subsection{Robustness to oscillation induced by RQH}

The sampling strategy strongly influences the robustness against limit cycle oscillations induced by the sampler. This kind of robustness can be successfully characterized by using the Describing Function (DF) technique, as proved in [56] and [58]. This method remains accurate as long as the linear part of the system provides enough filtering to attenuate the contribution of the high-order harmonics. For the context of this Chapter, the applicability hypothesis made by the DF hold, in Chapter 4 the case where the low-pass filter property of the system does not hold will be treated. To apply the DF method, the block scheme presented in Figure 2.1 can be rewritten as that in Figure 2.6, where $G_{o l}(s)=C(s) G(s)$ is the open-loop transfer function, and $G(s)=G_{p}(s) e^{-t_{d} s}$ includes the plant model and the network delay. The EG-ZOH block samples the signal according to the RQH law and holds the last value sampled until the switching conditions are fulfilled, then, a new sample is taken and held again. Thus, it is clear that the combination of the sampling strategy block and the $Z O H$ results in a non-linearity, and consequently it can be studied with the DF method.

It is well known that the condition for the existence of limit cycle in the system of Figure 2.6 is given by:

$$
G_{o l}(j \omega)=-\frac{1}{\mathcal{N}}, \quad \forall \omega,
$$

where $\mathcal{N}$ is the describing function of the non-linearity. The graphical interpretation of equation (2.6) is that the system does not present limit cycle if the plots of $G_{o l}(j \omega)$ and $-\frac{1}{\mathcal{N}}$ do not intersect. The DF for the proposed RQH sampling strategy, including the parameters $h$ and $\delta$, 
is given by the following equation (see Appendix B.1):

$$
\begin{gathered}
\mathcal{N}(A, h)=\frac{2 \delta}{A \pi}\left[\sum_{k=1}^{m} \sqrt{1-\left(\frac{\delta}{A}\left(k+\frac{h}{2 \delta}-\frac{1}{2}\right)\right)^{2}}+\right. \\
\left.\sum_{k=m+1}^{2 m} \sqrt{1-\left(\frac{\delta}{A}\left(2 m-k-\frac{h}{2 \delta}+\frac{1}{2}\right)\right)^{2}}\right]-j \frac{2 h m \delta}{A^{2} \pi},
\end{gathered}
$$

where $A$ is the amplitude of the sinusoidal oscillation and $m=\left\lfloor\frac{A}{\delta}-\frac{h}{2 \delta}+\frac{1}{2}\right\rfloor$, i.e. the maximum number of levels crossed by the oscillation. From this expression the SSOD and the RQ describing functions can be obtained by replacing $h=\delta$ and $h=0$ respectively. But the most interesting cases are found in the range $h \in] 0, \delta[$, where all the intermediate cases between SSOD and RQ appear.

Figure 2.7 depicts the shapes of $-1 / \mathcal{N}$ for different values of $h / \delta$. The locus of $-1 / \mathcal{N}$ are composed of several branches, one for each value of $m$. All the branches tend to fold and move towards the Real-axis as $h / \delta$ decreases. The case in Figure 2.7 where $h / \delta=1$ represents the negative inverse of $\mathcal{N}$ for SSOD, whose study was addressed, together with the case of the RQ sampler, by the authors in [58], where some measures were defined to characterize their robustness against limit cycle oscillations. Nevertheless, to take into account the intermediate cases between SSOD and RQ, presented in Figure 2.7 when $h / \delta \neq 1$, new robustness measures must be defined.

Similar to the classical gain and phase margins, gain margin to the non-linearity $\left(\gamma_{h / \delta}\right)$ has been defined as the increment in the $G_{o l}$ gain before reaching the intersection with $-1 / \mathcal{N}$ :

$$
\gamma_{h / \delta}=\frac{\left|-\frac{1}{\mathcal{N}\left(A_{\gamma}\right)}\right|}{\left|G_{o l}\left(j \omega_{\gamma}\right)\right|}
$$

where $\omega_{\gamma}$ and $A_{\gamma}$ are the values of $\omega$ and $A$ for which the quotient $\frac{\left|-\frac{1}{\mathcal{N}(A)}\right|}{\left|G_{o l}(j \omega)\right|}$ is minimum while fulfilling the condition $\arg \left(G_{o l}(j \omega)\right)=\arg \left(-\frac{1}{\mathcal{N}(A)}\right)$. This can be written in a compact form as:

$$
\left(\omega_{\gamma}, A_{\gamma}\right)=\arg \min _{(\omega, A)}\left(\frac{\left|-\frac{1}{\mathcal{N}(A)}\right|}{\left|G_{o l}(j \omega)\right|}: \arg \left(G_{o l}(j \omega)\right)=\arg \left(\frac{-1}{\mathcal{N}(A)}\right)\right) .
$$

On the other hand, the phase margin to the non-linearity $\left(\Phi_{h / \delta}\right)$ is the minimum amount of phase required by $G_{o l}$ to intersect the non-linearity while fulfilling the condition $\left|-\frac{1}{\mathcal{N}(A)}\right|=\left|G_{o l}(j \omega)\right|$. That is:

$$
\Phi_{h / \delta}=\arg \left(G_{o l}\left(j \omega_{\Phi}\right)\right)-\arg \left(-\frac{1}{\mathcal{N}\left(A_{\Phi}\right)}\right)
$$

where $\omega_{\Phi}$ and $A_{\Phi}$ are the values of $\omega$ and $A$ for which the difference $\arg \left(G_{o l}(j \omega)\right)-\arg \left(-\frac{1}{\mathcal{N}(A)}\right)$ is minimum while fulfilling the condition $\left|-\frac{1}{\mathcal{N}(A)}\right|=\left|G_{o l}(j \omega)\right|$. This can be written in a compact 

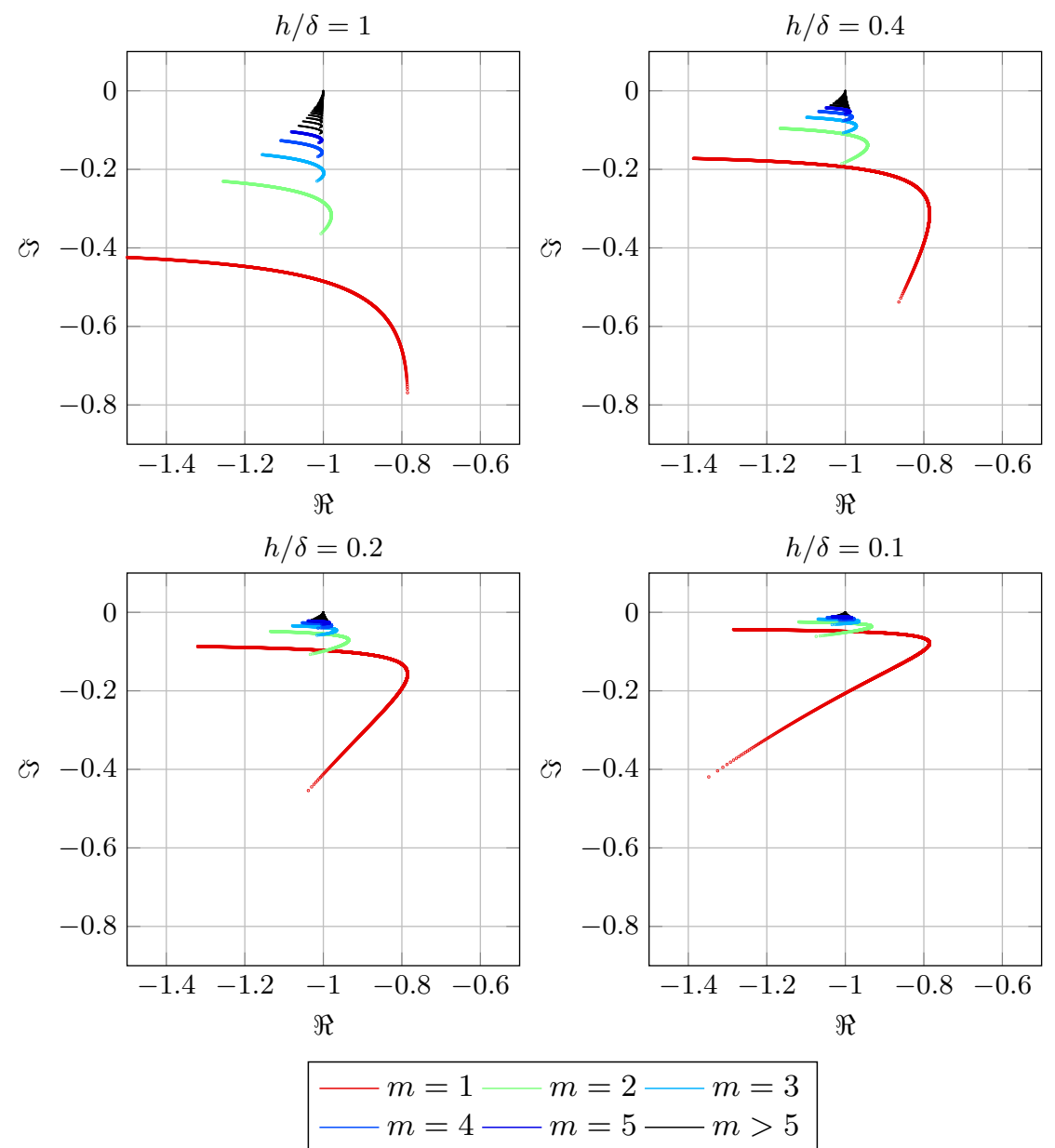

Figure 2.7: Shapes of $-1 / \mathcal{N}$ for RQH samplers with different values of $h / \delta$.

form as:

$$
\left(\omega_{\Phi}, A_{\Phi}\right)=\arg \min _{(\omega, A)}\left(\arg \left(G_{o l}(j \omega)\right)-\arg \left(-\frac{1}{\mathcal{N}(A)}\right):\left|-\frac{1}{\mathcal{N}(A)}\right|=\left|G_{o l}(j \omega)\right|\right) .
$$

It is important to note that, as proved in [56] and [58] for the cases SSOD and RQ, for PI controllers tuned with reasonable values of gain and phase margins the shape of $G_{o l}$ is such that the non intersection with the branch corresponding to $m=1$ guarantees no intersections with branches for $m>1$, and consequently, no intersection between $G_{o l}$ and $-1 / \mathcal{N}$ takes place. Therefore, in most of practical cases the limit cycles can be effectively eliminated by avoiding the intersection between $G_{o l}$ and the branch of $-1 / \mathcal{N}$ corresponding to $m=1$, and the margins $\gamma_{h / \delta}$ and $\Phi_{h / \delta}$ are measured with respect to this branch.

Figure 2.8 shows the margins $\gamma_{h / \delta}$ and $\Phi_{h / \delta}$ in the Nyquist and Nichols diagrams for a given system and a given value of $h / \delta$. In this figure only the branch $m=1$ of $-1 / \mathcal{N}$ has been represented. These robustness measures are easily visualized on Nichols chart because they are, 

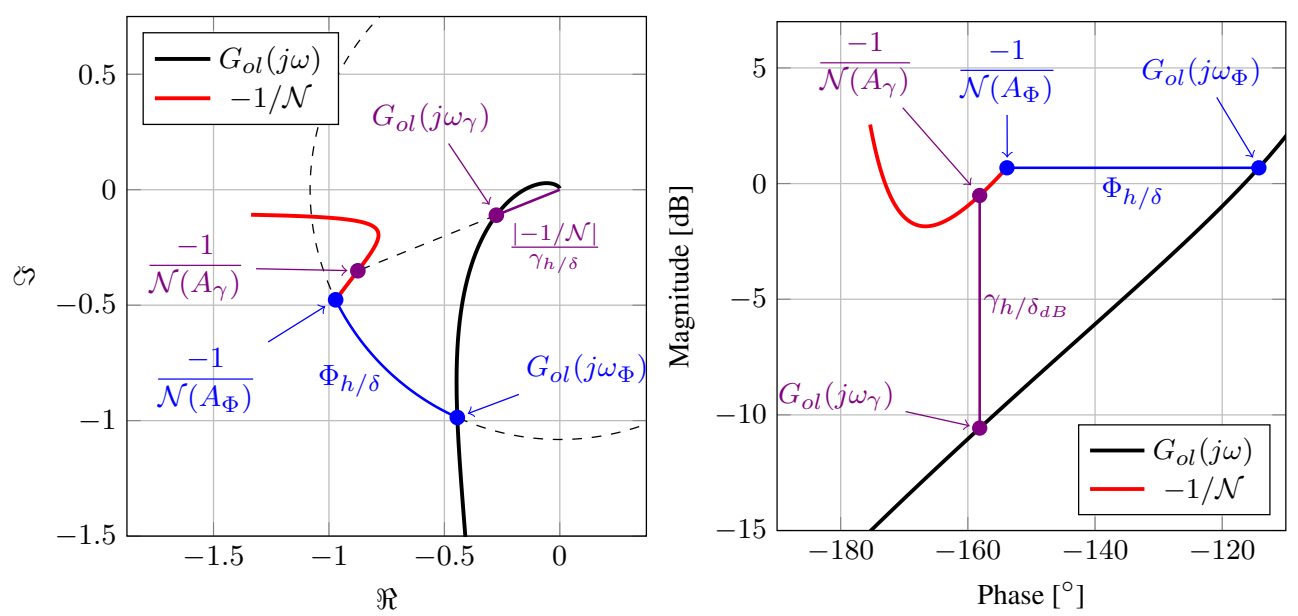

Figure 2.8: Gain and phase margins to the non-linearity $\left(\gamma_{h / \delta}, \Phi_{h / \delta}\right)$ for a given open-loop transfer function $G_{o l}$ in Nyquist and Nichols diagrams.

similar to the traditional gain and phase margins, the vertical and horizontal minimum distances from the $G_{o l}(j \omega)$ to the negative inverse of $\mathcal{N}$.

As postulated in [69], the condition for avoiding limit cycle oscillation results from a generalization of the Nyquist stability criterion by considering the describing function as a generalized gain. This generalization states that the entire Nyquist curve of the open-loop transfer function must encircle anticlockwise the critical points $(-1 / \mathcal{N}, 0)$ the number of times equal to the number of poles with positive real part in $G_{o l}$. Therefore, if neither the plant $G_{p}(s)$ nor the controller $C(s)$ have poles with positive real part, the Nyquist curve should not encircle the negative inverse of the describing function to avoid limit cycles. This fact allows defining the margins $\gamma_{h / \delta}$ and $\Phi_{h / \delta}$ to measure the distance between $-1 / \mathcal{N}$ and $G_{o l}$ when encircles are not required for stability. According to that, these margins can not be applied to plants whose poles have positive real part.

The proposed margins guard the traces of $-1 / \mathcal{N}$ against intersections with $G_{o l}(s)$ due to modeling errors or variations in the plant dynamics and network delay. If the open-loop transfer function for the nominal plant does not cross the boundary defined by $\gamma_{h / \delta}$ and $\Phi_{h / \delta}$, the system will remain without oscillations for a certain range of variation on the plant parameters. The magnitude of the admissible variations is strongly influenced by the structure of the plant model $G_{p}(s)$, so it has to be studied for each specific case. The robustness can be also affected by variation on the network delay, which is always present in the control system under study. The effect of variation on this parameter is addressed next.

\subsubsection{Network communication delay influence on the robustness margins}

The effect of the delay introduced by the communication network on the robustness margins is considered by including the term $e^{-t_{d} s}$ in the open-loop transfer function. However, variations on $t_{d}$ could degrade the robustness against limit cycle oscillations. To study the influence of 
this parameter on $\gamma_{h / \delta}$ and $\Phi_{h / \delta}$, consider a variation on $t_{d}: t_{d}^{\prime}=t_{d} \pm \alpha$. Then the open-loop transfer function is:

$$
G_{o l}^{\prime}(j \omega)=G_{o l}(j \omega) e^{\mp j \omega \alpha} .
$$

This equation can be expressed in terms of magnitude and phase as:

$$
\left\{\begin{array}{c}
\left|G_{o l}^{\prime}(j \omega)\right|=\left|G_{o l}(j \omega)\right| \\
\arg \left\{G_{o l}^{\prime}(j \omega)\right\}=\arg \left\{G_{o l}(j \omega)\right\} \mp \omega \alpha
\end{array},\right.
$$

from which it can be seen that variations on the network delay correspond to horizontal displacements of $G_{o l}(j \omega)$ in the Nichols diagram. Concretely, the resulting open-loop transfer function will approach to the negative inverse of the describing function traces as $t_{d}^{\prime}$ increases, worsening both $\gamma_{h / \delta}$ and $\Phi_{h / \delta}$. On the other hand, the reduction of $t_{d}^{\prime}$ improves both margins. Due to this effect, the higher value of admissible delays introduced by the network should be considered as $t_{d}$ to avoid the degradation of the margins due to the variation on this parameter.

It must be remarked that, depending on the process dynamics defined by $G_{p}(s)$, the network delay can be neglected because of its minor influence on $G_{o l}$, and consequently on the robustness margins. In those cases, the delay term can be omitted from the open-loop transfer function.

\subsection{Evaluation of classical tuning methods using the proposed mar- gins}

To illustrate the usefulness of the proposed margins they have been applied to study the robustness against limit cycle oscillations induced by PI controllers with transfer function

$$
C(s)=K_{p}\left(1+\frac{1}{T_{i} s}\right)
$$

tuned with well known methods when the controllers are used under the RQH sampling strategy scheme as that in Figure 2.1. The tuning methods selected for this study are Ziegler-Nichols [79], Cohen-Coon [18] and AMIGO [4]. PI controllers have been tuned for the batch of models presented below, which describe a wide range of behaviors that can be found in actual real systems. The dynamic responses of the models in the batch were approximated by First Order Plus Time Delay (FOPTD) models to obtain the parameters of their respective controller. The robustness margins $\gamma_{h / \delta}$ and $\Phi_{h / \delta}$ have been calculated in all cases. 


$$
\begin{aligned}
& G(s)=\frac{e^{-s}}{(T s+1)^{2}}, \\
& T=0.01,0.02,0.05,0.1,0.2,0.3,0.5,0.7,1 \text {, } \\
& 1.3,1.5,2,4,6,8,10,20,50,100,200,500 \\
& G(s)=\frac{1}{(s+1)(T s+1)^{2}}, \\
& T=0.05,0.1,0.2,0.5,2,5,10 \\
& G(s)=\frac{1}{(s+1)^{n}}, \\
& n=3,4,5,6,7,8 \\
& G(s)=\frac{1}{(s+1)(\alpha s+1)\left(\alpha^{2} s+1\right)\left(\alpha^{3} s+1\right)}, \\
& \alpha=0.1,0.2,0.3,0.4,0.5,0.6,0.7,0.8,0.9 \\
& G(s)=\frac{T e^{-L_{1} s}}{\left(T_{1} s+1\right)(T s+1)}, \\
& T_{1}+L_{1}=1, \quad T=1,2,5,10 \\
& L_{1}=0.01,0.02,0.05,0.1,0.3,0.5,0.7,0.9,1 \\
& G(s)=\frac{1-\alpha s}{(s+1)^{3}}, \\
& \alpha=0.1,0.2,0.3,0.4,0.5,0.6,0.7,0.8,0.9,1,1.1 \\
& G(s)=\frac{1}{(s+1)\left((s T)^{2}+1.4 s T+1\right)}, \\
& T=0.1,0.2,0.3,0.4,0.5,0.6,0.7,0.8,0.9,1
\end{aligned}
$$

The open-loop transfer functions, $G_{o l}(s)=C(s) G(s)$, for all the designs are represented in the Nyquist and Nichols diagrams in Figures 2.9 and 2.10, where the traces of $-1 / \mathcal{N}$ for one level oscillations, $m=1$, obtained for different ratios $h / \delta$ are also depicted. As commented before, the behavior of these traces with the reduction of the ratio $h / \delta$, in both Nyquist and Nichols diagrams, is to fold and tend to a straight line, horizontal on the real axis for the Nyquist diagram and vertical at $-180^{\circ}$ for the Nichols diagram, which corresponds to the RQ sampling.

From these figures some conclusions can be drawn. Firstly, the Ziegler-Nichols and CohenCoon methods offer some controllers which make the open-loop transfer function to intersect with the negative inverse of the DF for some values of $h / \delta$, and thus, these systems will oscillate when sampled with those strategies. Secondly, the AMIGO method offers controllers which avoid the intersection with $-1 / \mathcal{N}$ and provide the higher values of $\gamma_{h / \delta}$ and $\Phi_{h / \delta}$, therefore oscillations due to the RQH sampling will not take place, even for certain variations in the plant dynamic. 

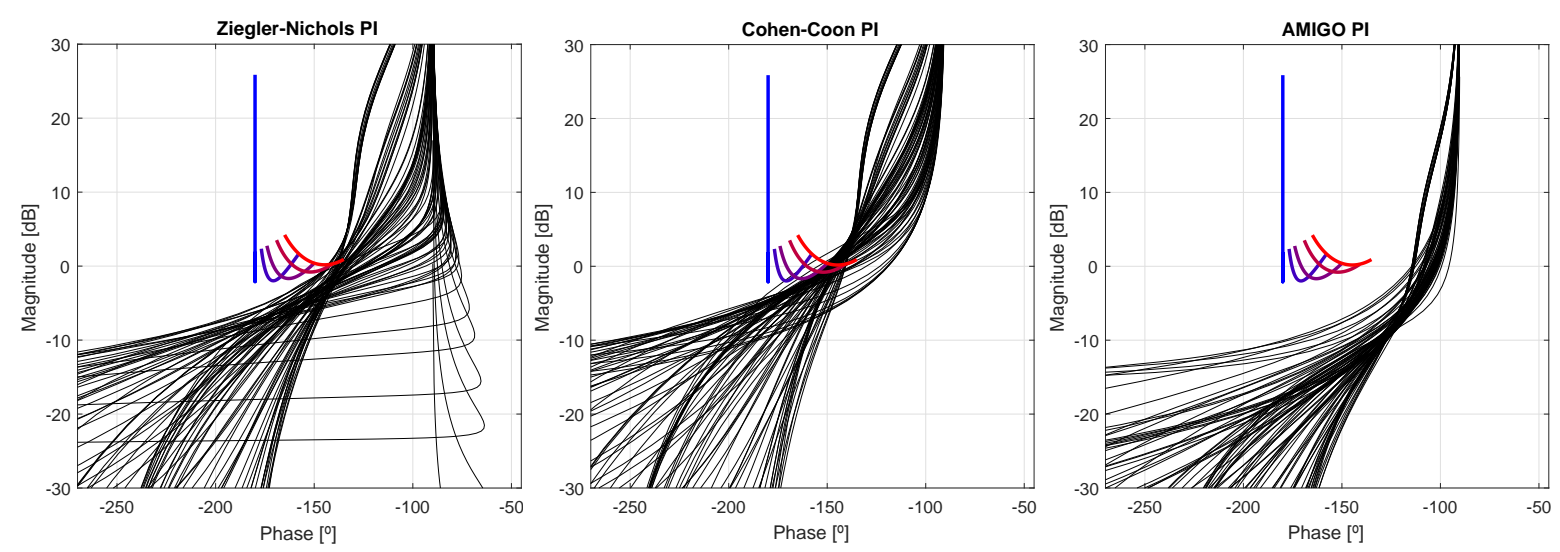

$-h / \delta=1(\mathrm{SSOD})-h / \delta=2 / 3-h / \delta=1 / 3-h / \delta=1 / 6-h / \delta=0(\mathrm{RQ})$

Figure 2.9: Nichols plots of the presented batch of processes with the specified controllers and the traces of $-1 / \mathcal{N}$ for $m=1$ with different values of $h / \delta$.
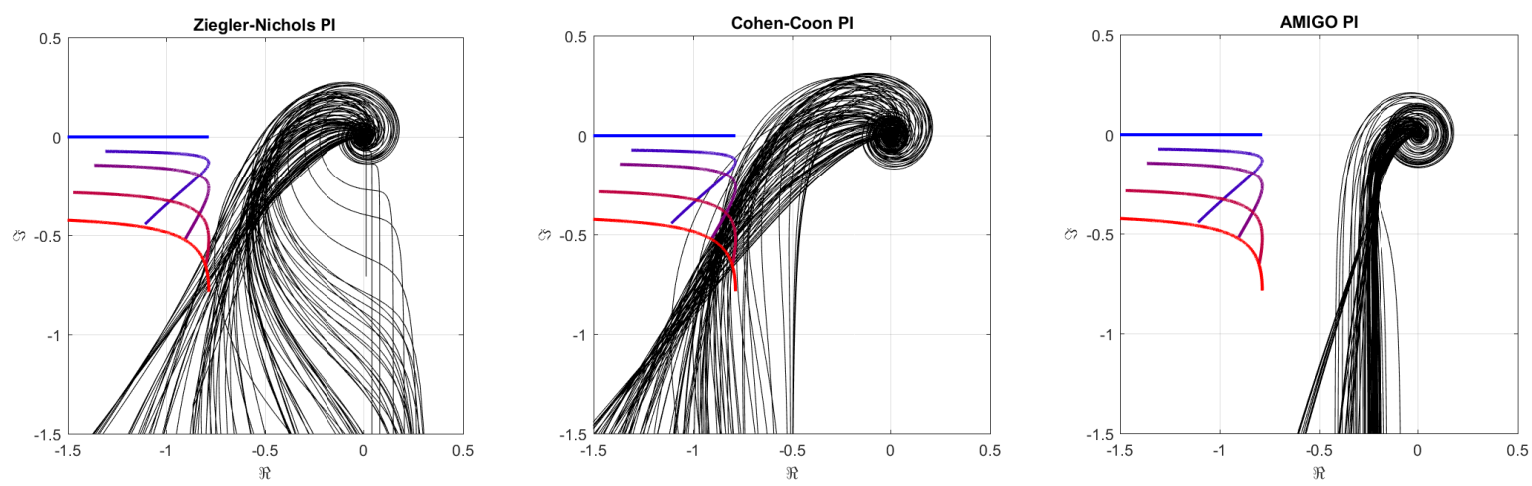

$-h / \delta=1(\mathrm{SSOD})-h / \delta=2 / 3-h / \delta=1 / 3-h / \delta=1 / 6-h / \delta=0(\mathrm{RQ})$

Figure 2.10: Nyquist plots of the presented batch of processes with the specified controllers and the traces of $-1 / \mathcal{N}$ for $m=1$ with different values of $h / \delta$.

In view of these results, to assure that limit cycle oscillations will not take place, the proposed margins must be checked once the controller is tuned. To evaluate the robustness of a concrete design for particular variations in the plant model, the magnitude of changes in the plan dynamic must be expressed in term of gain and phase variations of $G_{o l}$. If such variations are lower than the respective margins, then the design is robust enough. On the other hand, if as a consequence of changes in the dynamic behavior of the plant the margins $\gamma_{h / \delta}$ and $\Phi_{h / \delta}$ are surpassed, then instabilities will appear.

\subsubsection{Influence of $h / \delta$ on the robustness margins}

The margins $\gamma_{h / \delta}$ and $\Phi_{h / \delta}$ for different values of $h / \delta$ are shown in Figures 2.11-2.13. The results corroborate the preliminary observations from Figures 2.9 and 2.10. Firstly, it must be 

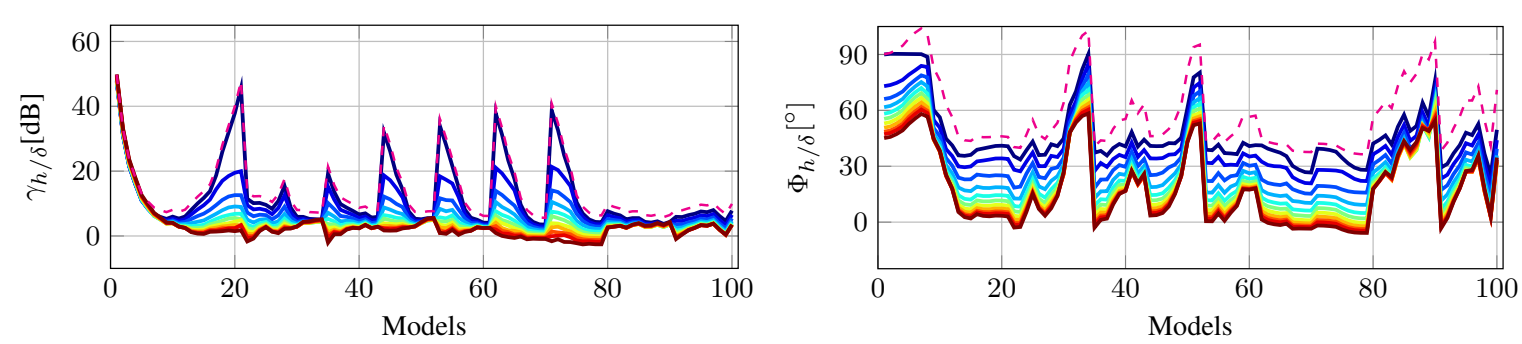

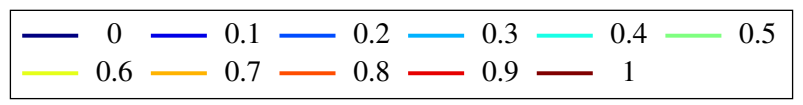

Figure 2.11: $\gamma_{h / \delta_{d B}}$ and $\Phi_{h / \delta}$ for several values of $h / \delta$ (solid colored lines) and classical gain and phase margins (dashed magenta line) with Ziegler-Nichols tuning method.
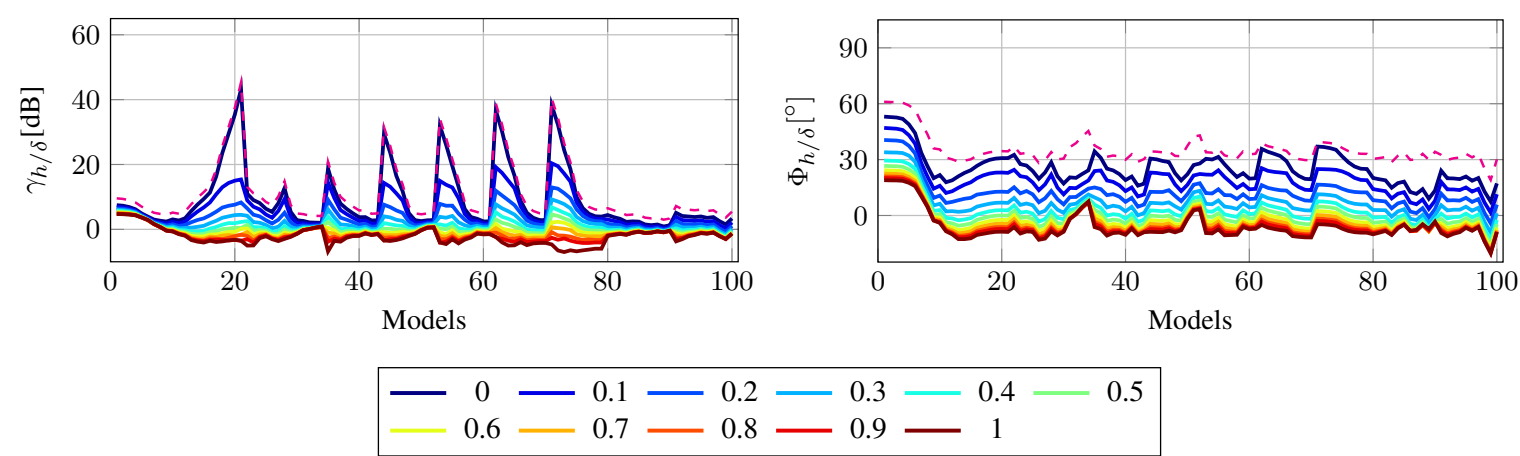

Figure 2.12: $\gamma_{h / \delta_{d B}}$ and $\Phi_{h / \delta}$ for several values of $h / \delta$ (solid colored lines) and classical gain and phase margins (dashed magenta line) with Cohen-Coon tuning method.

highlighted the good behavior of PI controllers tuned with AMIGO rules, which have positive values of margins for all the batch processes and all ratios $h / \delta$. It is also remarkable that for Ziegler-Nichols and Cohen-Coon methods, despite the fact of having reasonable values of classical phase and gain margins, which are also represented in the figure with dashed lines, for high values of $h / \delta$ some systems present negative values of $\gamma_{h / \delta}$ and $\Phi_{h / \delta}$, i.e. it exists an intersection between the open-loop transfer function and $-1 / \mathcal{N}$. Particularly critic are the results of the Cohen-Coon method whose margins are negative for most of the processes when $h / \delta$ is greater than 0.4 .

Beyond the results obtained for each tuning method, the previous study reveals that the reduction of $h / \delta$ tends to increase both $\Phi_{h / \delta}$ and $\gamma_{h / \delta}$, being ultimately similar to the classical gain and phase margins when the RQ sampler is considered.

\subsubsection{Influence of controller's parameters on the robustness margins}

Concerning the influence of the controller parameters on the proposed margins, it is worth noting that both $K_{p}$ and $T_{i}$ modify the relative position of $G_{o l}(s)$ with regard to $-1 / \mathcal{N}$. The effect of varying these parameters can be easily observed in the Nichols diagram because changes in $K_{p}$ and $T_{i}$ produce vertical and/or horizontal displacements on $G_{o l}(s)$. 

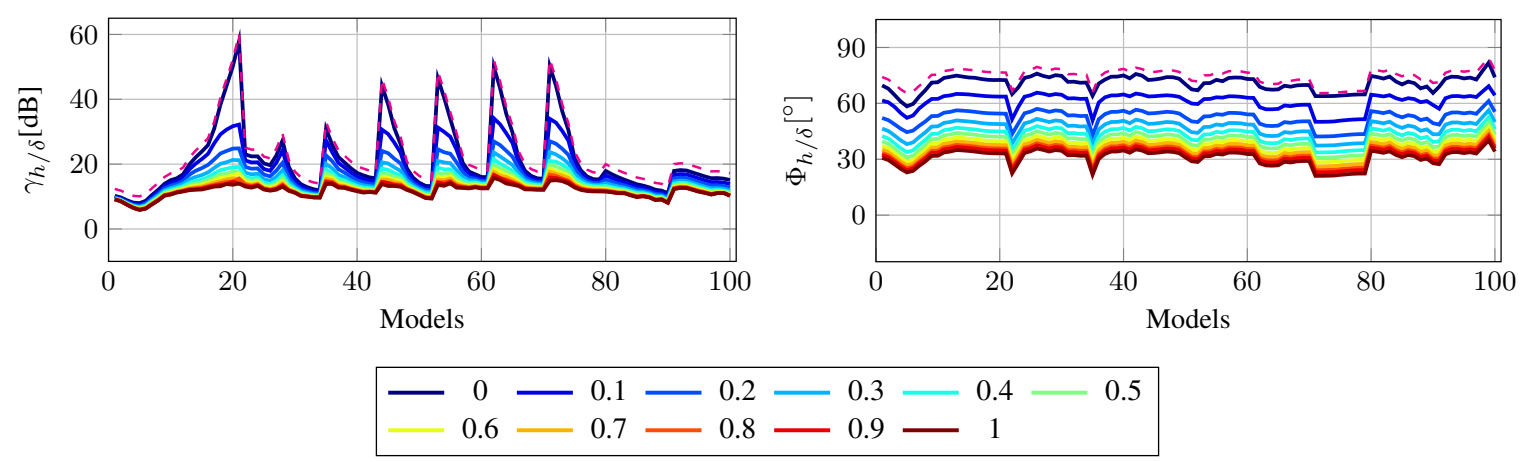

Figure 2.13: $\gamma_{h / \delta_{d B}}$ and $\Phi_{h / \delta}$ for several values of $h / \delta$ (solid colored lines) and classical gain and phase margins (dashed magenta line) with AMIGO tuning method.

The magnitude and phase expressions for a PI controller with transfer function in equation (2.14) are:

$$
\begin{gathered}
|C(j \omega)|=K_{p} \sqrt{1+\left(\frac{1}{\omega T_{i}}\right)^{2}} . \\
\arg \{C(j \omega)\}=\arctan \left(-\frac{1}{\omega T_{i}}\right)
\end{gathered}
$$

From these expressions it can be seen that the proportional gain produces a vertical displacement of $G_{o l}(j \omega)$ in the Nichols diagram. Concretely the $G_{o l}(j \omega)$ moves down as $K_{p}$ decreases, which, due to the shape of $G_{o l}(j \omega)$ depicted in Figure 2.9, implies the improvement of both margins. On the other hand, the variation on the integral time produces both a vertical and horizontal displacement of $G_{o l}(j \omega)$. It can be seen that by increasing $T_{i}$ a displacement downwards and to the right of $G_{o l}(j \omega)$ takes place in the Nichols diagram, which implies an improvement of both margins.

According to these results, by detuning PI controllers (reducing $K_{p}$ and/or increasing $T_{i}$ ) both margins can be raised. This behavior of $\Phi_{h / \delta}$ and $\gamma_{h / \delta}$ with respect to the controller parameters is qualitatively similar to that of the classical gain and phase margins, therefore, the detuning of the controller improves all the four margins at the expense of getting slower closed-loop responses.

\subsection{Simulation examples}

In this section, the main issues related with the RQH sampler presented in this chapter are illustrated through simulation examples.

Example 2. This example shows the influence of $h / \delta$ in the event generation. The number of generated events in RQH - PI(s) loops strongly depends on the choice of the sampling parameters, i.e. hysteresis $h$ and quantification $\delta$. In section 2.3.1 a study about the effect on the event generation of the ratio $h / \delta$ for samplers which conduct to the same steady-state error was 


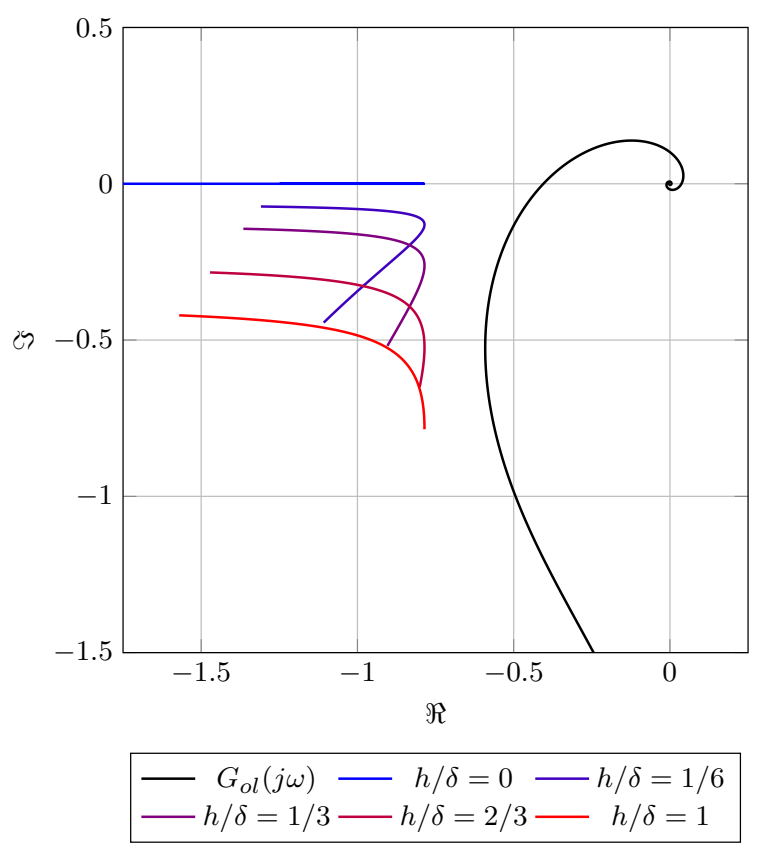

Figure 2.14: Nyquist diagram of $G_{o l}(j \omega)$ and the inverse negative of the DF corresponding to several samplers.

presented. In the following example, the influence of this choice is shown by comparing SSOD and $R Q H$ samplers.

Consider a process whose transfer function is described by:

$$
G_{p}(s)=\frac{e^{-0.3 s}}{(s+1)(0.7 s+1)} .
$$

The network communication delay is tested to have a latency $t_{d}=0.15$ seconds. Thus, the whole model to be considered for the tuning is described by:

$$
G(s)=\frac{e^{-0.45 s}}{(s+1)(0.7 s+1)} .
$$

A PI controller has been tunned following Ziegler-Nichols tuning rules, obtaining $K_{p}=1.52$ and $T_{i}=2.58$. This controller does not make the open-loop transfer function intersect the traces of the inverse negative for any ratio $h / \delta$, therefore, it avoids limit cycle oscillations induced by the sampler. This fact can be corroborated in Figure 2.14 where the Nyquist diagram of $G_{o l}(j \omega)$ and the inverse negative of the DF for several ratios $h / \delta$ have been represented. As it can be seen, no intersection exists between $G_{o l}(j \omega)$ and the $-1 / \mathcal{N}$ traces.

The measurement noise present in this loop is observed to have a peak-to-peak amplitude of 0.07 units and the admissible maximum steady-state error to provide a correct functioning is $e_{s s}=0.18$. With these requirements, following the guidelines in section 2.3, the SSOD is chosen to have $\delta=0.18$ to minimize the event generation and assuring the accomplishment of 

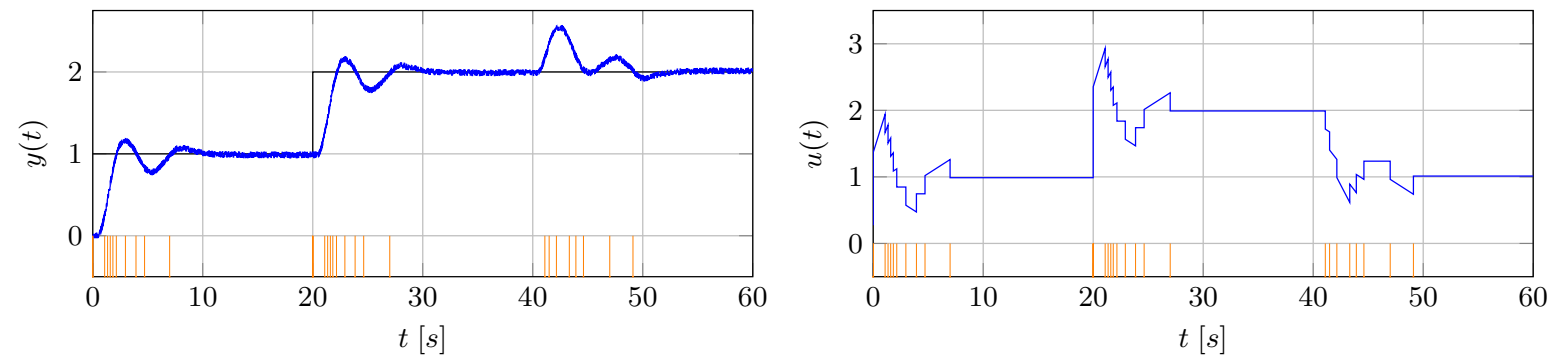

Figure 2.15: Controlled output $y$ and control action $u$ for the system containing a SSOD sampler, which results in a number of events generated $n_{e v}=31$, marked in orange.
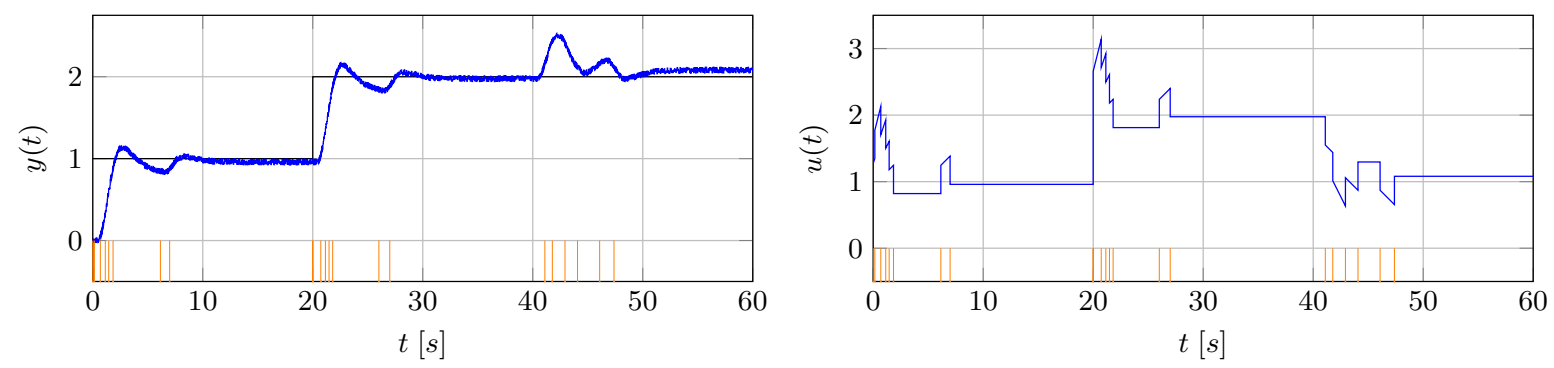

Figure 2.16: Controlled output $y$ and control action $u$ for the system containing a RQH sampler, which results in a number of events generated $n_{e v}=22$, marked in orange.

the specifications. With regard to the RQH sampler, to avoid event generation due to noise, the hysteresis is chosen to be $h=0.08$, and, to fulfill the maximum $e_{s s}$ criterion, $\delta=0.28$.

Two experiments have been performed with the same process and controller but changing the sampler in the loop. The experiments consist in two unitary step changes at the reference input and a unitary step change in the disturbance input at different times. The results of the experiments can be seen in Figures 2.15 and 2.16 for the SSOD and RQH samplers respectively.

Regarding both figures, there are not remarkable differences which could make one sampler preferable over the other. In terms of the controlled output both reach the steady-state regime in about 10 seconds. The unique difference resides in the number of events generated, which for the case of the SSOD is $n_{e v}=31$ and for the $R Q H$ is $n_{e v}=22$, reducing in a significant manner the number of generated events.

This example proves that by choosing a RQH sampler over a SSOD, the system performance is not significantly affected and the number of events generated is lower. Nevertheless, this event generation reduction implies that the control action bumps produced by a change of magnitude $\delta$ at the input of the controller, $\delta_{u}=K_{p} \delta$, is higher for the RQH than for the SSOD. In this example, these control action bumps for the SSOD are $\delta_{u}=0.274$ and for the RQH are $\delta_{u}=0.426$.

Example 3. This example shows the usefulness of DF approach on predicting the oscillation induced by the RQH sampler. As commented before, the ratio $h / \delta$ has a strong influence on the appearance of limit cycle because it changes significantly the shape of the inverse negative of the 

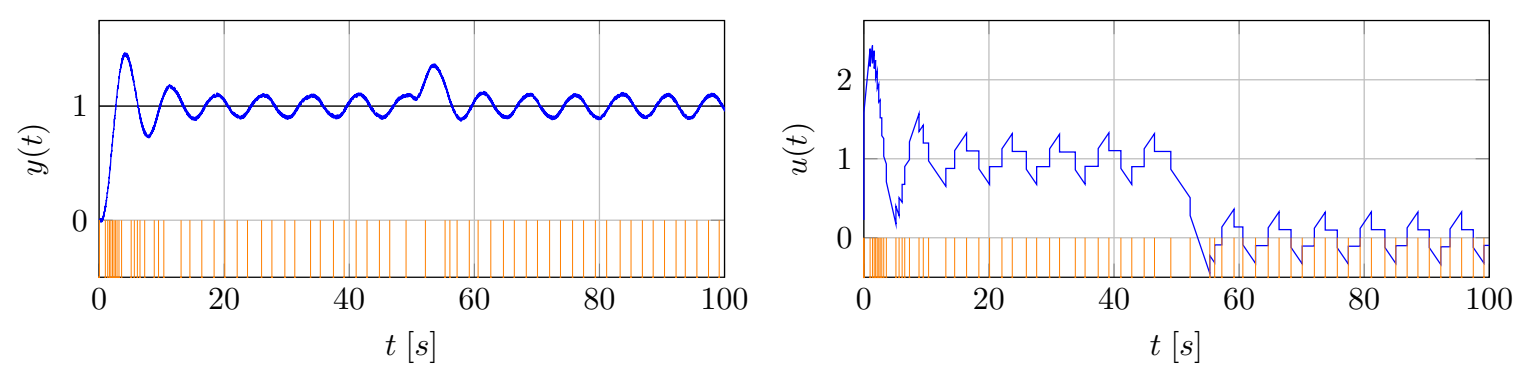

Figure 2.17: Controlled output $y$ and control action $u$ of a system with a RQH sampler with $h / \delta=0.4286$ in the loop, which leads to an oscillatory response.

DF and, therefore, the robustness margins $\gamma_{h / \delta}$ and $\Phi_{h / \delta}$. In this example, the influence of the ratio $h / \delta$ on the robustness is highlighted.

Consider a process whose transfer function is described by:

$$
G_{p}(s)=\frac{1-0.1 s}{(s+1)^{3}}
$$

The network communication delay is small enough with respect to the system dynamics, so it can be neglected. A PI controller has been tuned according to Cohen-Coon tuning rules, resulting in $K_{p}=1.612$ and $T_{i}=1.938$.

The measurement noise in the loop is observed to have a peak-to-peak amplitude of 0.03 units and the maximum admissible steady-state error is $e_{s s}=0.1$. From this specifications two $R Q H$ samplers will be designed and tested in the loop.

For the first sampler, consider a very conservative approach to avoid the event generation due to noise, in which the hysteresis will be selected to be twice the observed peak-to-peak amplitude $h=0.06$ and the quantification $\delta$ is selected to meet the maximum admissible $e_{s s}, \delta=0.14$, resulting in a sampler with ratio $h / \delta=0.4286$.

This system has been simulated and its temporal response to a unitary step change in the reference and disturbance inputs is presented in Figure 2.17. As it can be seen, the resulting system response is oscillatory. This is justified in the previous section because, even if the classical gain $\gamma_{c p}=6.5 \mathrm{~dB}$ and phase $\Phi_{c g}=30.37^{\circ}$ margins for most of continuous applications provide enough robustness, the gain $\gamma_{h / \delta}=-0.4 d B$ and phase $\Phi_{h / \delta}=-1.98^{\circ}$ margins to the non-linearity obtained for the $R Q H$ with $h / \delta=0.4286$ indicate the existence of an intersection between $G_{o l}(j \omega)$ and $-1 / \mathcal{N}$ as corroborated in Figure 2.18a, and therefore, it exists a limit cycle.

A more suitable RQH sampler is obtained by choosing the hysteresis $h=0.04$ and the $\delta$ to fulfill the $e_{s s}$ requirement, $\delta=0.16$, resulting in a ratio $h / \delta=0.25$, which is lower than in the precedent case. With this new RQH sampler, the proposed robustness margins are recalculated, and the respective gain and phase margins, $\gamma_{h / \delta}=1.15 d B$ and $\Phi_{h / \delta}=5.58^{\circ}$, are obtained, which indicate that the oscillation condition is not satisfied, and then, limit cycle oscillations will not take place. This fact is shown in Figure $2.18 b$ where it can be seen that the intersection between $G_{o l}(j \omega)$ and $-1 / \mathcal{N}$ is avoided. The avoidance of the apparition of limit cycle oscillations has 


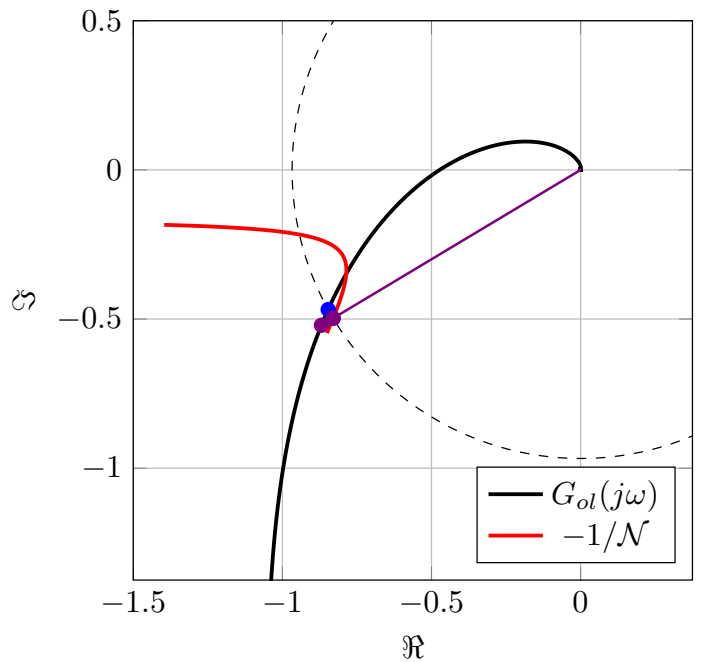

(a) $\mathrm{RQH}$ with $h / \delta=0.4286$.

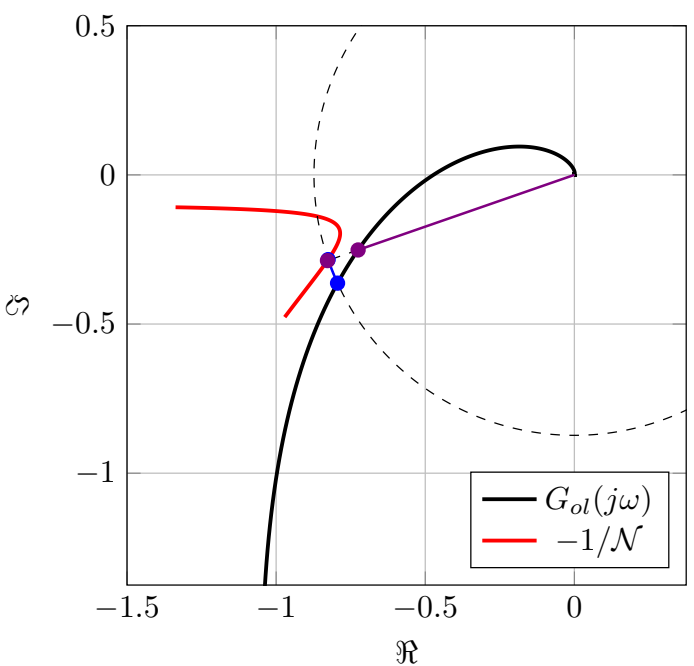

(b) $\mathrm{RQH}$ with $h / \delta=0.25$.

Figure 2.18: Nyquist diagram of $G_{o l}$ with the inverse negative of the DF of its respective sampler.
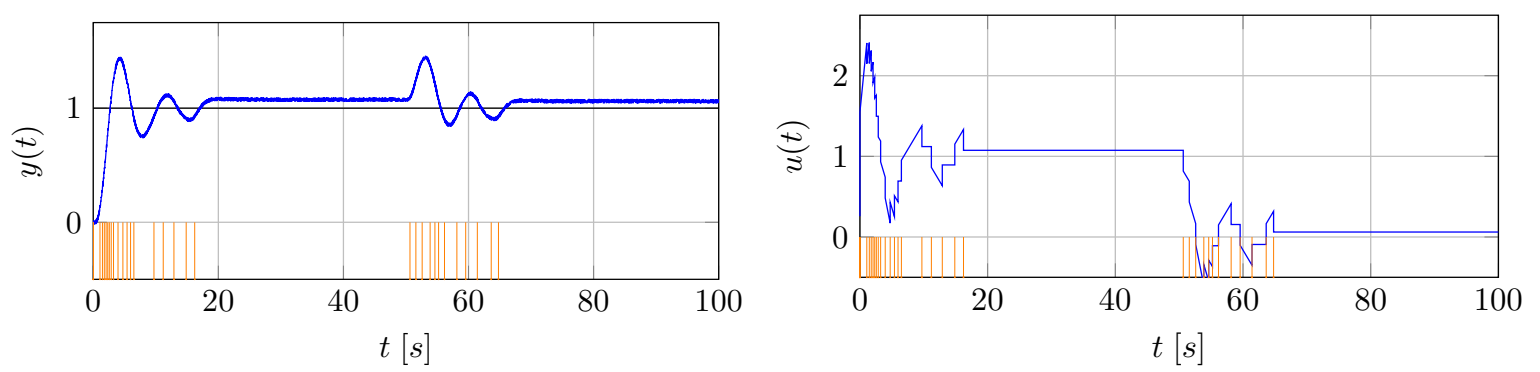

Figure 2.19: Controlled output $y$ and control action $u$ of a system with a RQH sampler with $h / \delta=0.25$ in the loop, which avoids limit cycle oscillations.

also been tested through a simulation with the same conditions as in the precedent case, which is presented in Figure 2.19, where the system response to a unitary step change in the reference and disturbance input has been presented. As expected, this system does not present limit cycle oscillations, which shows that, effectively, a reduction on the ratio $h / \delta$ of the sampler lowers the robustness requirements of the system to avoid limit cycle oscillations.

Example 4. In an industrial environment, errors in the process model can appear due to several common causes, such as noisy measurement, few data for identification or non-linear behaviors, among others. The robustness margins serve to cope with the modeling error as well as with possible variations in the plant dynamic. Studying these margins for the worst case scenario can be insightful to determine the actual robustness taking into account the uncertainty in the plant parameters. Furthermore, it is also possible to express the margins in term of admissible variation in the parameters of the model, even though this analysis is valid only for the model structure that is being studied. This example illustrates these ideas. 


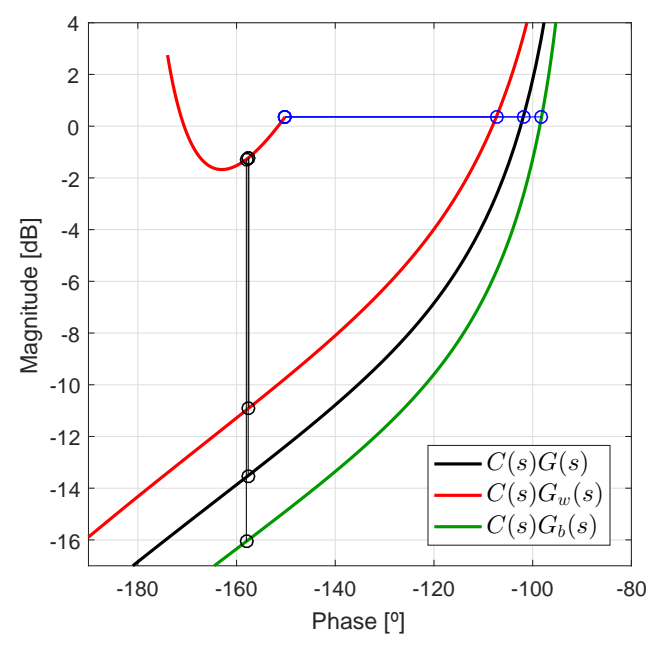

Figure 2.20: Modeled system with the best and worst case scenarios due to uncertainty with their respective robustness margins to the non-linearity.

Consider a process with nominal model given by the following transfer function:

$$
G(s)=\frac{e^{-0.2 s}}{(s+1)^{4}}
$$

whose gain, delay and poles have been obtained with a reliability of $\pm 5 \%$. A PI controller has been tuned using AMIGO tuning rules, obtaining $K_{p}=0.281$ and $T_{i}=2.41$. A RQH sampling with ratio $h / \delta=1 / 3$ has been applied.

Because of the model uncertainties, the worst and best case scenarios, from the robustness point of view, are respectively:

$$
G_{w}(s)=\frac{1.05 e^{-0.21 s}}{(s+0.95)^{4}} \text { and } G_{b}(s)=\frac{0.95 e^{-0.19 s}}{(s+1.05)^{4}} .
$$

These models have been evaluated with the controller. The Nichols diagrams are depicted in Figure 2.20, where it can be seen that the margins to the non-linearity vary from $\gamma_{h / \delta}=9.7 d B$ and $\Phi_{h / \delta}=42.8^{\circ}$ for the worst case to $\gamma_{h / \delta}=14.8 \mathrm{~dB}$ and $\Phi_{h / \delta}=52^{\circ}$ for the best case, being $\gamma_{h / \delta}=12.3 \mathrm{~dB}$ and $\Phi_{h / \delta}=48.3^{\circ}$ the values obtained for the nominal model. In this case, the achieved margins are good enough to consider the system robust against oscillations induced by the sampler, even considering the effect of the model uncertainties.

In addition to the previous study, the admissible variation of each nominal parameter without provoking limit cycle oscillation can be calculated. For this specific case, the gain can be increased until 4.11, the delay until 6.98 seconds and the multiple poles can be decreased to 0.75. This kind of analysis allows expressing the robustness in terms of parameter variation, however, the results depend on the structure of $G_{p}(s)$. 


\subsection{Conclusions}

In this chapter, an alternative to either regular quantification (RQ) or symmetric-send-on-delta (SSOD) sampling strategies for event-based PI control systems has been presented. The proposed solution consists in an intermediate case between both strategies, attained by regulating the ratio between the quantification step and the quantifier hysteresis.

This solution presents several advantages regarding RQ and SSOD. Firstly, it avoids sending events, and its associated data, through the network generated by changes in the sampled signal due to noise. In addition, it reduces the number of events needed to perform the control while still being reactive to significant changes in the state of the system.

To evaluate the robustness against limit cycles of the proposed sampling strategy the describing function technique has been used, obtaining the regions that induce limit cycle oscillations on the system. From the knowledge of these regions, gain and phase margins to limit cycles induced by the RQH sampler have been defined.

Using these margins, the suitability of classical tuning methods for continuous systems, such as Ziegler-Nichols, Cohen-Coon and AMIGO, can be evaluated when used for tuning controllers under RQH sampling strategy. An extensive simulation study shows that, even with good classical gain and phase margins provided by these methods, limit cycle oscillations can still be induced by the sampler if the proposed margins are negative.

The influence of the controller's parameters, network delay and model uncertainty on the proposed margins has been studied. The results reveal that the effect of varying these parameters on the new margins are similar to that obtained on classical gain and phase margins.

Additionally, the guidelines to select proper sampler parameters from noise and steady-state error specifications have been provided, and its influence on the limit cycle oscillations apparition and on the event generation has been addressed and highlighted through several examples. 



\title{
Tuning Procedure for $P I$ controllers under RQH sampling
}

\begin{abstract}
In this chapter, a tuning procedure is proposed for event based PI controllers with Regular Quantization with Hysteresis (RQH) sampling law. The RQH is a generalization of Symmetric-Send-on-Delta (SSOD) strategy which decreases the robustness requirements to avoid limit cycle oscillations and reduce the number of events needed for control, improving the overall performance of PI controllers in networked control systems. The tuning procedure takes into account not only classical robustness margins, but it also takes advantage of some specific robustness measures to avoid limit cycle oscillations induced by the sampler. As the robustness analysis depends on the Describing Function (DF) method, a study evaluating the effect of high order harmonics is provided, showing the validity of the tuning procedure. Some examples are included in which the usefulness of the tuning procedure is shown.
\end{abstract}

\subsection{Introduction}

Event-Based Control (EBC) of continuous system is getting more and more attention recently [38]. This is due to some of the advantages that EBC offers, such as providing a better management of the data flow through the digital networks on distributed control systems, reducing the data drop out in the form of package losses and decreasing the delays caused by the communication. These benefits are a direct consequence of the EBC data transmission policy, which consists in sending data only when significant changes are detected on the state of the system and not periodically as in most of classical control loops. In networked control systems the EBC approach may be considered among the most promising control approaches, indeed, in [20] its importance in modern factory automation has been recently highlighted.

In [2] a summarized but extensive study about the main contributions on EBC in the last two decades is presented. The results in that paper reveal scarce of investigation on Event-Based 
PID with respect to other control approaches such as Model-based, Predictive or State-feedback control. PID, however, is used in most of the industrial control applications. Due to the high sensitivity of the derivative action to the measurement noise, the PI control is the dominant form of the PID in use today, [5]. This dominance in the industrial environment over other advanced control strategies like MPC, was reflected in a survey conducted among the industrial committee members of the International Federation of Automatic Control published in [62]. The versatility of PID algorithms also allows them to remain important in the modern context of Industry 4.0 as it was highlighted in [39], where the necessity of introducing new features to the PID algorithms in order to adapt them to the high connectivity between devices through wired and wireless communication networks that this paradigm offers was also pointed out. The adaptation of PID to the paradigm of Event-Based Control can contribute to its use in these new application scenarios.

In EBC systems, the event generation policy is of paramount importance because it is in charge of generating and sending the events that regulate the execution of the controller algorithm, which, as shown in [30], determines the performance and behavior of sampled control systems. The most used event generation techniques, mainly because of their simplicity of implementation, are the ones based on the signal quantification, like the send-on-delta (SOD) sampling technique, which is based on sending new events when the sampled signal changes in more than a threshold $\delta$. The effectiveness of this strategy has been tested in terms of control performance and communication reduction, $[19,50]$.

To the knowledge of the author, the use of SOD in PID control loops was introduced by Årzén [3] to reduce the use of CPU in embedded control systems without degrading significantly the system performance. To do that, the sensor is sampled periodically but the control algorithm is executed only if the error signal crosses preset thresholds. Some further works were focused on solving the problems raised by Årzén, mainly related with the calculation of the integral of error when the time between samples increases. It should be noted in particular the works of Durand [22, 23] and Vasyutynskyy [77, 76]. Recent works have extended the Årzén proposal to fractional order systems. Concretely, the implementation issues of the discrete event-based fractional order controllers have been addressed for two different control algorithms: FO-IMC [46] and FO-PID $[13,15,14]$. Because the aim of these papers is to reduce the computation effort of the control algorithms, their main contribution is the development of control routines computationally more efficient. However, no tuning procedures have been developed for these algorithms taking into account the effect of the SOD sampler, and the use of tuning methods for continuous controllers is suggested instead.

With similar principles than in SOD sampling, in [10] a sampling strategy known as SymmetricSend-On-Delta (SSOD) was presented, being the main characteristic traits the inclusion of a hysteresis with the same magnitude than the quantification threshold $\delta$ and having fixed switching levels. Several works have been published with regard to SSOD sampling in loops with a PI controller, concerning tuning procedures, identification and application cases. In [11], a tuning procedure for PI controllers in SSOD sampling was presented taking into account first order plus time delay (FOPTD) models, and some rules were designed by minimizing the $1 \%$ settling time 
of the closed-loop response. SSOD sampler has also been used for identification, for instance, in [70] a system identification procedure was proposed. In [49], event-based controllers with a SSOD sampling strategy were applied to the inside air temperature control of the greenhouse production process.

In [60] and [56], some tuning methods for PI controllers with SSOD sampler have been developed. Additionally, in [61] a unified design of a SSOD based PID and Smith predictor for self-regulating and integral processes was investigated. The approach followed in these publications was based on some robustness margins to avoid limit cycles that were obtained by applying the Describing Function (DF) technique, whose use allowed to introduce the classical concepts of phase and gain margins in the design of this kind of EBC. The DF is a well known analysis tool for Wiener-Hammerstein non-linear systems introduced in the 30s, and posteriorly presented in [35]. Several variants based on this technique were presented, e.g. the dual input DF [78], the sampled or discrete DF [33] and the fractional order DF [73]. A lot of literature can be found about this method, for example in [25] and [47].

Another sampling strategy based on the signal quantization is the Regular Quantification (RQ) sampling strategy, which is an alternative to SSOD event generation, and consists in sending new data whenever the value of the sampled signal is a multiple of the quantization threshold $\delta$. A comparative study between SSOD and RQ strategies was presented in [58]. Due to the lack of hysteresis in the RQ sampler, the measurement noise can produce bursts of events, and thus, bursts of data to transmit, which is the main disadvantage of RQ with respect to SSOD. Nevertheless, the inclusion of the hysteresis, as in the case of SSOD, forces the controller to fulfill higher robustness requirements to avoid limit cycle oscillations.

Taking these effects of the hysteresis into account, in [42] a new sampling strategy called Regular Quantization with Hysteresis (RQH) was presented. This sampling strategy consists of a quantification with fixed thresholds and with variable hysteresis, which can be chosen freely, and depending on these parameters choice the intermediate cases between the RQ and SSOD appear. In that work the event generation for the same process reactivity was characterized and the robustness against limit cycle oscillations studied, introducing new gain and phase robustness margins to the presence of this kind of oscillations.

Using these new margins, it has been proved that in general the tuning methods for continuous PI do not provide good enough results when applied to PI controllers with RQH sampling since either the limit cycles are not avoided or, conversely, extremely robust controllers are obtained which is an indicator that faster behavior can be achieved. This fact evidenced the necessity for developing new tuning algorithms for this kind of control systems taking into account the effects of the RQH sampler in order to improve the trade-off between robustness and speed of response.

In this chapter, a tuning procedure for PI controllers within a loop in which the error signal is sampled according to the RQH principles is proposed. The procedure takes into account classical robustness measures as gain and phase margin, as well as it includes specific robustness measures, also in terms of gain and phase, to the oscillations induced by the sampler in the 
loop, which were presented in [42]. The tuning procedure and the obtained margins are tested in simulation for several processes which prove the tuning procedure applicability. Additionally, the validation of any controller placed within a structure with an RQH sampling is studied by taking into account additional harmonics which have an effect on the robustness measures.

The chapter is organized as follows. Section 3.2 presents the main characteristics and advantages of the RQH sampling strategy and highlights the necessity of a specific tuning method. Section 3.3 proposes a tuning procedure for the controller placed in a loop with a RQH sampler. This tuning procedure takes into account both classical and specific to the non-linearity robustness margins. Section 3.4 studies the validation of controllers placed in this kind of loops by considering the effect of high order harmonics. Finally, the conclusions about this work are drawn.

\subsection{Problem statement}

The typical networked control system in which event generators like the $\mathrm{RQH}$ are placed is presented in Figure 2.1 in the previous Chapter. In that figure, the controller and process are denoted by the blocks $C(s)$ and $G_{p}(s)$ respectively, the applied sampler, or event generator, is represented by the EG block, the $\mathrm{ZOH}$ block is a zero-order hold and the network's delay is modeled by $\exp \left(-t_{d} s\right)$. The reference signal to track is denoted by $y_{r}$, the controlled output by $y$ and the disturbance signal by $p$. The controller is assumed to be placed close to the actuator. The measured signal $e^{*}$ of the error $e$ is sent by the event generator through the communication network and the $\mathrm{ZOH}$ block keeps in $\bar{e}$ the last value sent until new data arrive. This control scheme was first proposed in [10] considering that the EG block was a SSOD and the controller a PI. Instead of the SSOD, in this work the RQH sampling will be used in the EG block.

The RQH sampler is defined essentially by two parameters, the quantization level $\delta>$ 0 and the hysteresis $h$ that can be freely selected as long as $0 \leq h \leq \delta$, being the ratio $h / \delta$ the characteristic parameter that defines most of the sampler properties. The relation between an input $x$ and its output $\bar{x}$ of the RQH sampler is presented in Figure 3.1. In this figure the RQ or SSOD samplers input-output relationship can be obtained by fixing $h=0$ or $h=\delta$ respectively, being the $\mathrm{RQH}$ a more general strategy that embraces both, which consequently, presents characteristics that are a trade-off between RQ and SSOD. Namely, immunity to generate events caused by noise in the signal, low event generation for the same reactivity to changes and lesser robustness requirements for the controller to avoid limit cycle oscillations than the SSOD sampler.

To select the proper parameters for the RQH sampler a simple procedure was presented in [42]. Firstly, the hysteresis $h$ is selected slightly greater than the peak-to-peak amplitude of the measure noise to prevent it from generating events. Secondly, the parameter $\delta$ is selected to assure a certain reactivity to significant changes on the system, which are defined by the steady-state error $e_{s s}$. The RQH sampling, as well as SSOD and RQ strategies, introduces a steady-state error on the controlled output $y$, this is due to the existence of a dead band around 


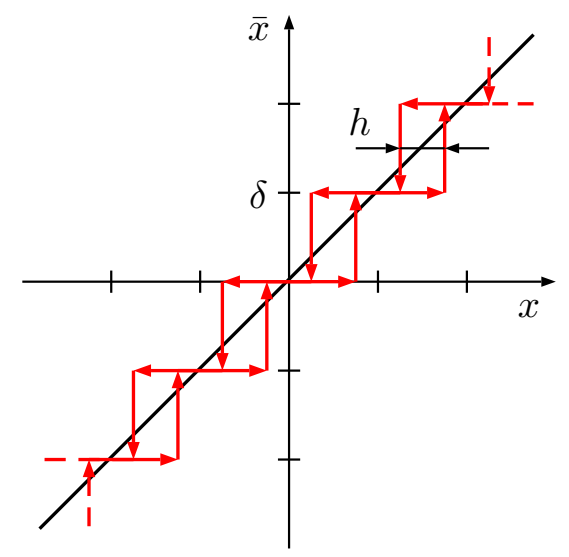

Figure 3.1: Relationship between input $x$ and output $\bar{x}$ for the RQH sampling strategy.

$e=0$ in which the sampler will send $\bar{e}=0$ because the thresholds are not surpassed. The steadystate error in RQH samplers is defined by $e_{s s}=(\delta+h) / 2$. If $h$ has been previously chosen to avoid event generation due to the measurement noise, then $\delta$ can be obtained directly from this expression to fulfill the $e_{s s}$ requirement. Additionally, the ratio $h / \delta$ of the $\mathrm{RQH}$ sampler has a big influence on the number of generated events, which increases proportionally with this ratio, thus, making the $e_{s s}$ of the system to be the maximum admissible $e_{s s}$, by means of maximizing $\delta$, decreases to the minimum possible the number of events generated for a given change in the signal to track.

Once the ratio $h / \delta$ is defined, the $\mathrm{RQH}$ can be characterized in terms of robustness using the Describing Function (DF) technique. To apply the DF technique, as it has been said in the previous Chapter, the block scheme presented in Figure 2.1 can be rewritten as that in Figure 2.6, in which the network delay, the control and process transfer functions are grouped in $G_{o l}(s)=G_{p}(s) C(s) e^{-t_{d} s}$, which is the open-loop transfer function of the system, and the rest in the block EG-ZOH. This last block presents the same behavior as the original blocks: it samples the signal and holds its value until new samples are taken. Therefore, the EG-ZOH results in a non-linearity which can be studied with the DF method.

The condition for the existence of limit cycle in the system presented in Figure 2.6 is given by:

$$
G_{o l}(j \omega)=-\frac{1}{\mathcal{N}}, \quad \forall \omega
$$

where $\mathcal{N}$ is the describing function of the non-linearity. Graphically, if it exists an intersection between the open-loop transfer function and the inverse negative of the DF, the system will present limit cycle oscillations. The DF for the RQH sampling strategy was presented in [42] 
and is given by the following equation:

$$
\begin{gathered}
\mathcal{N}(A, h)=\frac{2 \delta}{A \pi}\left[\sum_{k=1}^{m} \sqrt{1-\left(\frac{\delta}{A}\left(k+\frac{h}{2 \delta}-\frac{1}{2}\right)\right)^{2}}+\right. \\
\left.\sum_{k=m+1}^{2 m} \sqrt{1-\left(\frac{\delta}{A}\left(2 m-k-\frac{h}{2 \delta}+\frac{1}{2}\right)\right)^{2}}\right]-j \frac{2 h m \delta}{A^{2} \pi}
\end{gathered}
$$

where $A$ is the amplitude of the sinusoidal oscillation and $m=\left\lfloor\frac{A}{\delta}-\frac{h}{2 \delta}+\frac{1}{2}\right\rfloor$ is the maximum number of levels crossed by the oscillation.

The traces of $-1 / \mathcal{N}$ are composed of several branches, one for each value of $m$. As the ratio $h / \delta$ decreases, all the branches tend to fold and move towards the Real-axis, being a horizontal line when $h / \delta=0$. In Figure 3.2 the locus of $-1 / \mathcal{N}$ for different values of $h / \delta$ is represented. The case where $h / \delta=1$ represents the negative inverse of $\mathcal{N}$ for SSOD and several studies about its robustness have already been presented $[58,44]$.
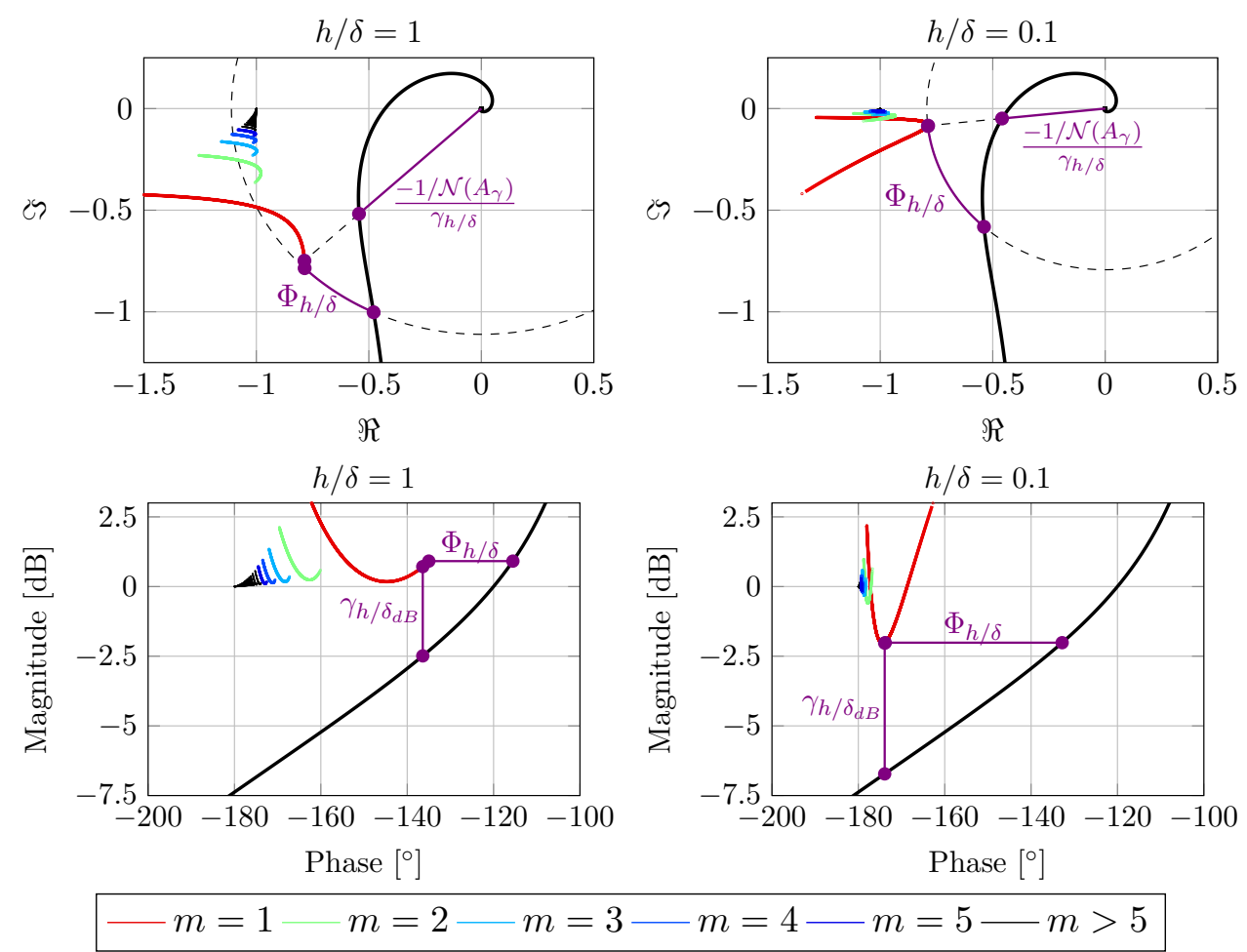

Figure 3.2: Shapes of $-1 / \mathcal{N}$ for RQH samplers with different values of $h / \delta$ in Nyquist and Nichols diagrams with the gain and phase margins to the non-linearity $\gamma_{h / \delta}$ and $\Phi_{h / \delta}$.

An important remark is that for PI controllers tuned with reasonable robustness margins the shape of $G_{o l}$ is such that the non intersection with the branch corresponding to $m=1$ guarantees to avoid intersections with branches for $m>1$, and therefore, no intersection between $G_{o l}$ and $-1 / \mathcal{N}$ takes place, avoiding limit cycle oscillations. 
Using the expression of the DF presented in equation (3.2), in [42], a study of the robustness against limit cycles of certain classical tuning methods was presented for a batch of processes. To that end, a gain $\gamma_{h / \delta}$ and phase $\Phi_{h / \delta}$ margins to the non-linearity were defined, revealing that some methods cannot be applied for certain ratios $h / \delta$ without presenting limit cycle oscillations, and other methods present an extremely robust behavior which can be an indicator that faster controllers can be tuned. These margins are presented in Figure 3.2 in Nyquist and Nichols diagrams in violet.

In addition, unlike the classical phase and gain margins, an important consideration to be taken into account is that the robustness margins to the non-linearity $\gamma_{h / \delta}$ and $\Phi_{h / \delta}$ usually provide poor information about the closed-loop performance. This is due to the fact that they are not measured with regard to a fixed point, instead, these measures depend on the non-linearity and on the open-loop transfer function shapes. In a similar way, assuring some classical gain and phase margins, or using classical tuning rules, does not guarantee to avoid limit cycle oscillations induced by the sampling because it has not been taken into account in the original design. Thus, in this chapter a tuning procedure which takes into account both classical margins and these new robustness margins to the non-linearity in the design procedure is developed.

\subsection{Tuning procedure for RQH based PI}

From the study presented in [42] and as it has been commented before, tuning methods for continuous PI are not entirely valid for designing controllers with RQH sampling strategy since $\Phi_{h / \delta}$ and $\gamma_{h / \delta}$ must be checked afterwards to ensure the avoidance of limit cycles. In this section, a tuning procedure for PI controllers will be introduced taking into account classical robustness measures and these new specific measures. The only restriction for the calculation of the new margins is the usage of the DF technique, which assumes the process to be filtering enough to neglect the high order harmonics effect. This means that the method can be used for a wide range of processes, having either sub-damped or over-damped responses including time delays or non minimum phase.

Before applying the tuning procedure, the parameters $h$ and $\delta$ that define the RQH describing function, and significantly affect the robustness measures, must be selected taking into account the admissible steady-state error $\left(e_{s s}\right)$ and the peak-to-peak amplitude of the measurement noise, as commented in section 3.2. It is important to keep in mind that higher values of $h / \delta$ affect negatively the amount of events generated, which will be greater than the number of events generated with intermediate values of $h / \delta$. Besides, the increase of this ratio affects in an inversely proportional way the controller's speed to attain the same margins $\Phi_{h / \delta}$ and $\gamma_{h / \delta}$.

Once the parameters $\delta$ and $h$ have been selected, the shape of $-1 / \mathcal{N}$ is defined, and the PI controller tuning procedure can be addressed. The goal of the tuning procedure proposed in this section is to obtain the controller that minimize the IAE (Integral of Absolute value of the Error) index of the disturbance response while fulfilling the requirements on gain and phase 
margins. This tuning problem can be expressed as follows:

$$
\begin{array}{cl}
\underset{K_{p}, T_{i}}{\operatorname{minimize}} & I E \\
\text { subject to } & \gamma_{c g} \geq \gamma_{c g_{r}}, \quad \Phi_{c p} \geq \Phi_{c p_{r}}, \\
& \gamma_{h / \delta} \geq \gamma_{h / \delta_{r}}, \quad \Phi_{h / \delta} \geq \Phi_{h / \delta_{r}}
\end{array}
$$

where $\gamma_{c g}$ and $\Phi_{c p}$ are the classical gain and phase margin, and the sub-index $r$ refers to the required value of each parameter. It is well known that for non-oscillatory responses the minimization of $I A E$ and $I E$ (Integral of the error) are equivalents. However, using the later is preferable because the $I E$ is directly related with the controller's parameters trough the integral gain $\left(K_{i}=K_{p} / T_{i}\right)$ which facilitates the solution of the optimization problem.

As commented before, the RQH sampling causes the apparition of a steady-state error, and thus, that the integral of the error signal will tend to infinite. However, in Appendix D.2 it has been proven that, with reasonable parameters, the integral of the sampled error signal is similar to the integral of the error signal in a continuous system, i.e. to the IE index.

Before describing the tuning procedure, it is important to note that the following conditions must be met in order to fulfill the margins restrictions in equation (3.3):

$$
\begin{aligned}
& \text { Classical gain margin }\left\{\begin{array}{l}
\left|G\left(j \omega_{c g}\right)\right|\left|C\left(j \omega_{c g}\right)\right| \leq 1 / \gamma_{c g_{r}} \\
\arg \left(G\left(j \omega_{c g}\right)\right)+\arg \left(C\left(j \omega_{c g}\right)\right)=-\pi
\end{array}\right. \\
& \text { Classical phase margin }\left\{\begin{array}{l}
\left|G_{o l}\left(j \omega_{c p}\right)\right|=1 \\
\arg \left(G_{o l}\left(j \omega_{c p}\right)\right) \leq-\pi-\Phi_{c p_{r}}
\end{array}\right. \\
& h / \delta \text { gain margin }\left\{\begin{array}{l}
\left|G\left(j \omega_{\gamma}\right)\right|\left|C\left(j \omega_{\gamma}\right)\right| \leq \frac{1}{\gamma_{h / \delta_{r}}}\left|-\frac{1}{\mathcal{N}\left(A_{\gamma}\right)}\right| \\
\arg \left(G_{o l}\left(j \omega_{\gamma}\right)\right)=\arg \left(-\frac{1}{\mathcal{N}\left(A_{\gamma}\right)}\right)
\end{array}\right. \\
& h / \delta \text { phase margin }\left\{\begin{array}{l}
\left|G_{o l}\left(j \omega_{\Phi}\right)\right|=\left|-\frac{1}{\mathcal{N}\left(A_{\Phi}\right)}\right| \\
\arg \left(G_{o l}\left(j \omega_{\Phi}\right)\right) \leq \arg \left(-\frac{1}{\mathcal{N}\left(A_{\Phi}\right)}\right)-\Phi_{h / \delta_{r}}
\end{array}\right.
\end{aligned}
$$

Where $G(s)=G_{p}(s) e^{-t_{d} s}$ is considered to take into account the process transfer function $G_{p}(s)$ and the communication delay modeled by $e^{-t_{d} s}$.

The precedent equations define a whole set of controllers whose margins will be at least the required values or greater. The goal is to find the controller with maximum $K_{i}=K_{p} / T_{i}$ (minimum $I E)$ in this set.

The tuning procedure consists of 3 steps: 
1. Find the set of all the PI controllers that can be obtained for a given process.

2. Tune each controller obtained from step 1 according to the gain margin and detune those that do not fulfill the requirements on $\Phi_{c p}, \gamma_{h / \delta}$ or/and $\Phi_{h / \delta}$.

3. Finally, choose among all the resulting controllers the one with minimum $I E=K_{p} / T_{i}$.

Next, these steps are described in detail.

Step 1 The tuning procedure starts by obtaining the range of possible values for $\omega_{c g}$. Since for a PI controller $\arg (C(j \omega)) \in\left[-\frac{\pi}{2}, 0\right]$, the application of the equation (3.4b) implies that $\arg \left(G\left(j \omega_{c g}\right)\right) \in\left[-\pi,-\frac{\pi}{2}\right]$. Thus, the range of $\omega_{c g}$ can be directly obtained from the phase response of $G(j \omega)$ as the values of $\omega$ within the boundaries defined by $\arg (G(j \omega))=-\pi$ and $\arg (G(j \omega))=-\frac{\pi}{2}$. For this range of $\omega_{c g}$ a regular griding is defined. Each item in the grid corresponds to a PI controller whose parameter will be calculated according to step 2 .

Step 2 This step is applied to each item in the grid of $\omega_{c g}$ obtained in step 1 . The value of $T_{i}$ can be calculated using the equation of the PI phase

$$
\arg (C(j \omega))=\arctan \left(T_{i} \omega\right)-\frac{\pi}{2}
$$

that combined with equation $(3.4 \mathrm{~b})$ result in,

$$
T_{i}=\frac{1}{\omega_{c g}} \tan \left(-\arg \left(G\left(j \omega_{c g}\right)\right)-\frac{\pi}{2}\right) .
$$

Once $T_{i}$ has been obtained, the corresponding $K_{p}$ can be calculated. $K_{p}$ is firstly obtained to fulfill the restriction on $\gamma_{c g}$. For a PI controller, the modulus can be expressed as:

$$
|C(j \omega)|=K_{p} \frac{\sqrt{1+\left(\omega T_{i}\right)^{2}}}{\omega T_{i}} .
$$

Thus, from equation $(3.4 \mathrm{a})$ it is obtained:

$$
K_{p} \leq \frac{\omega_{c g} T_{i}}{\gamma_{c g_{r}}\left|G\left(j \omega_{c g}\right)\right| \sqrt{1+\left(\omega_{c g} T_{i}\right)^{2}}}
$$

As the objective is to optimize the IE index, the maximum gain $K_{p}$ within all the possible values defined by equation (3.11) will be considered. That is:

$$
K_{p}=\frac{\omega_{c g} T_{i}}{\gamma_{c g_{r}}\left|G\left(j \omega_{c g}\right)\right| \sqrt{1+\left(\omega_{c g} T_{i}\right)^{2}}}
$$

At this point, the controller fulfills the classical gain margin restriction. To meet the rest of margin conditions, equations (3.5a) to (3.7b) are checked and the controller is conveniently 
detuned if needed. This is based on the fact that reducing $K_{p}$ implies a radial shrinking of $G_{o l}(j \omega)$ in the Nyquist diagram, or a downward displacement in the Nichols diagram. Consequently, the controller's detuning improves all the robustness margins.

In order to satisfy the classical phase margin requirement, equations in (3.5) must be fulfilled. Considering that in the construction of $G_{o l}$ the values of $K_{p}$ and $T_{i}$ previously calculated are used, the current margin $\Phi_{c p}$ is obtained. If $\Phi_{c p} \geq \Phi_{c p_{r}}$, no modifications on $K_{p}$ have to be done. However, if this requirement is not fulfilled, the frequency which will become the new $\omega_{c p}$ is obtained from equation (3.5b), forcing the equality

$$
\arg \left(G_{o l}\left(j \omega_{c p}\right)\right)=-\pi-\Phi_{c p_{r}}
$$

A detuning factor $k$ is then introduced to fulfill the equation (3.5a), whose value can be calculated as:

$$
k=\frac{1}{\left|G_{o l}\left(j \omega_{c p}\right)\right|} .
$$

Once $k$ is obtained, the controller gain is recalculated as $k K_{p}$.

It is important to highlight that $k$ shrinks the shape of $G_{o l}(j \omega)$ in the Nyquist diagram and consequently all the robustness margins are risen. Therefore, the conditions in (3.4) hold true and the design meets both gain and phase margins.

To fulfill the restrictions on $\gamma_{h / \delta}$ and $\Phi_{h / \delta}$ the procedure is similar to the one used with the classic margins. Firstly, the margin $\gamma_{h / \delta}$ is calculated and compared with $\gamma_{h / \delta_{r}}$. If $\gamma_{h / \delta}<\gamma_{h / \delta_{r}}$, then $k$ must be recalculated. From the definition of $\gamma_{h / \delta}$ it is worth noticing that for $k G_{o l}(j \omega)$ the factor $k$ can be introduced as follows:

$$
\left(\omega_{\gamma}, A_{\gamma}\right)=\arg \min _{(\omega, A)}\left(\frac{\left|-\frac{1}{\mathcal{N}(A)}\right|}{k\left|G_{o l}(j \omega)\right|}: \arg \left(G_{o l}(j \omega)\right)=\arg \left(-\frac{1}{\mathcal{N}(A)}\right)\right) .
$$

Since in the previous equation $k$ does not affect the condition $\arg \left(G_{o l}(j \omega)\right)=\arg \left(-\frac{1}{\mathcal{N}(A)}\right)$ and $\frac{\left|-\frac{1}{\mathcal{N}(A)}\right|}{\left|G_{o l}(j \omega)\right|}$ is just scaled by $1 / k$, then the frequency $\omega_{\gamma}$ and $A_{\gamma}$ will remain invariable as $k$ changes. Thus, the new value of $k$ can be easily obtained from equation (3.6a):

$$
k=\frac{1}{\gamma_{h / \delta_{r}}}\left|-\frac{1}{\mathcal{N}\left(A_{\gamma}\right)}\right| \frac{1}{\left|G_{o l}\left(j \omega_{\gamma}\right)\right|} .
$$

The last margin to check is the phase margin to the non-linearity, $\Phi_{h / \delta}$. If its value is greater than $\Phi_{h / \delta_{r}}$, then no modifications have to be done to the controller. On the other hand, if $\Phi_{h / \delta}<\Phi_{h / \delta_{r}}$, the parameter $K_{p}$ must be recalculated. Consider the openloop transfer function given by $k G_{o l}(j \omega)$, this parameter $k$ can be introduced within the 


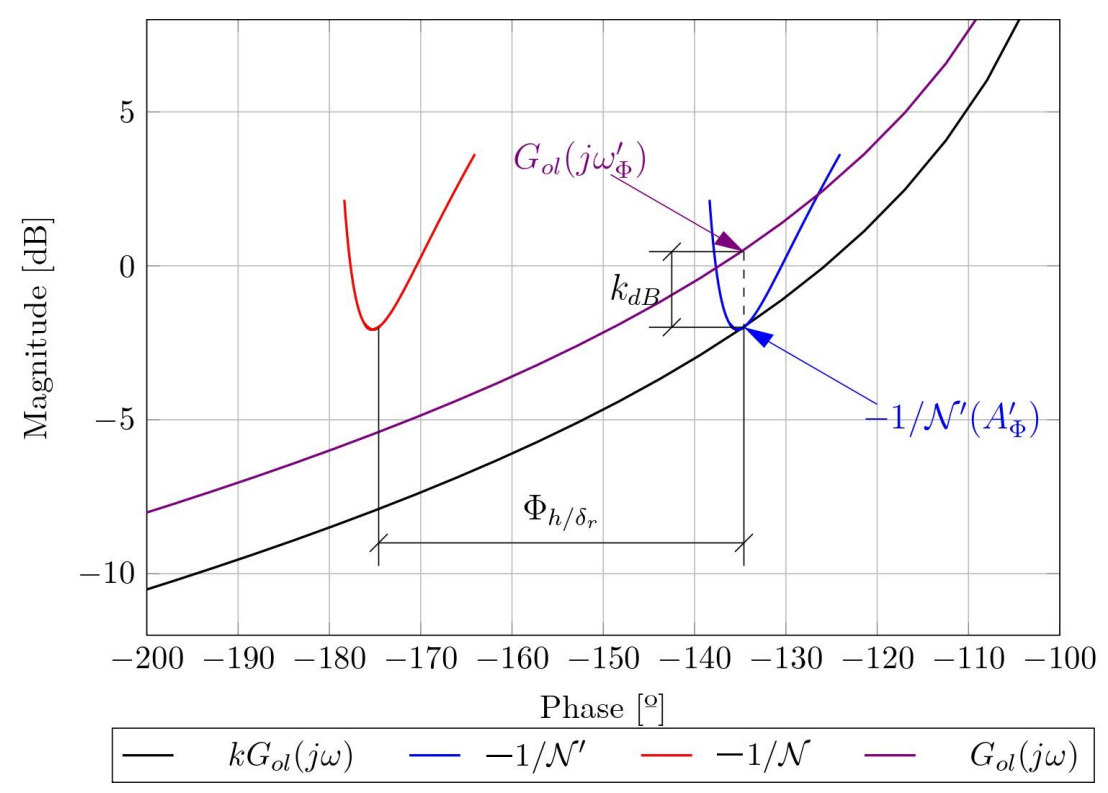

Figure 3.3: Graphical interpretation of the detuning procedure to fulfill the $\Phi_{h / \delta_{r}}$ margin.

definition of the phase margin to the non-linearity as:

$$
\left(\omega_{\Phi}, A_{\Phi}\right)=\arg \min _{(\omega, A)}\left(\arg \left(k G_{o l}(j \omega)\right)-\arg \left(-\frac{1}{\mathcal{N}(A)}\right):\left|-\frac{1}{\mathcal{N}(A)}\right|=k\left|G_{o l}(j \omega)\right|\right) .
$$

In the previous expression the condition $\left|-\frac{1}{\mathcal{N}(A)}\right|=k\left|G_{o l}(j \omega)\right|$ depends on the value of $k$. This means that, unlike the cases of $\omega_{\gamma}$ and $A_{\gamma}, \omega_{\Phi}$ and $A_{\Phi}$ depend on $k$. The new values of $\omega_{\Phi}$ and $A_{\Phi}$ in which the phase margin will be measured once the detuning factor is introduced are calculated as:

$$
\left(\omega_{\Phi}^{\prime}, A_{\Phi}^{\prime}\right)=\arg \max _{(\omega, A)}\left(\left|G_{o l}(j \omega)\right|-\left|-\frac{1}{\mathcal{N}^{\prime}(A)}\right|\right)
$$

where $-1 / \mathcal{N}^{\prime}(A)=-1 / \mathcal{N}(A) \cdot 1 / \Phi_{h / \delta_{r}}$. Then the detuning factor can be calculated as:

$$
k=\left|-\frac{1}{\mathcal{N}^{\prime}\left(A_{\Phi}^{\prime}\right)}\right| \frac{1}{\left|G_{o l}\left(j \omega_{\Phi}^{\prime}\right)\right|} .
$$

The graphical interpretation of this procedure is represented in Figure 3.3. The detuning factor $k$ is calculated to guarantee zero phase margin between $k G_{o l}$ and $-1 / \mathcal{N}^{\prime}$, which is an image of $-1 / \mathcal{N}$ whose phase has been displaced $\Phi_{h / \delta_{r}}$. Consequently, the minimal distance in phase between $k G_{o l}$ and $1 / \mathcal{N}$, or in other words, the phase margin, is $\Phi_{h / \delta_{r}}$.

Step 3 Finally, as a result of the preceding steps a set of controllers defined by pairs $\left(K_{p}, T_{i}\right)$ is obtained. Among them, the one that minimizes the $I E$ index is selected as the resulting 
controller, which, for PI controllers, is the one that maximizes the ratio $K_{p} / T_{i}$.

Remark 1. In some applications, an important issue to consider is the wear of the actuator, which could be reduced by limiting the control action bumps produced by the steps of magnitude $\delta$ introduced by the RQH sampler in the input of the controller. For a PI controller these bumps have amplitude $\delta_{u}=K_{p} \delta$. Using this equation it is possible to obtain the maximum $K_{p}$ for given values of $\delta$ and admissible control action bumps $\delta_{u_{\max }}: K_{p_{\max }}=\delta_{u_{\max }} / \delta$. Then, all the proportional gains obtained in step 2 that surpass $K_{p_{\max }}$ must be limited to this value.

Remark 2. Networked systems often contain communications delays, modeled in Figure 2.1 by the exp $\left(-t_{d} s\right)$ block. In the presented procedure this delay must be added to the process transfer function delay if it exists. Introducing delay in the loop reduces all the studied robustness margins, therefore, not considering it in the process transfer function $\left(G(s)=G_{p}(s) \exp \left(-t_{d} s\right)\right)$ could lead to a potential loss of robustness, causing the apparition of limit cycle oscillations or even instability. Thus, if this communication delay is known, it has to be considered into the process transfer function to perform the tuning procedure.

Remark 3. This tuning method can be applied to other type of controllers as long as the separation between linear and non-linear part presented in Figure 2.6 is kept. For example, for a PID controller the equations (3.8) and (3.10) result in:

$$
\begin{gathered}
\arg \{C(j \omega)\}=\arctan \left(\frac{\omega T_{i}\left(N T_{i} / T_{d}+1\right)}{N T_{i} / T_{d}-\left(\omega T_{i}\right)^{2}(1+N)}\right)-\arctan \left(\frac{\omega T_{i}}{N T_{i} / T_{d}}\right)-\frac{\pi}{2}, \\
|C(j \omega)|=K_{p} \frac{\sqrt{\left(\omega T_{i}\left(N T_{i} / T_{d}+1\right)\right)^{2}+\left(N T_{i} / T_{d}-\left(\omega T_{i}\right)^{2}(1+N)\right)^{2}}}{\omega T_{i} \sqrt{\left(\omega T_{i}\right)^{2}+\left(N T_{i} / T_{d}\right)^{2}}}
\end{gathered}
$$

respectively, and it has to be taken into account that the range for the controller's phase is $\left[-\frac{\pi}{2}, \frac{\pi}{2}\right]$, changing the expressions derived from (3.4)-(3.7). In this case, as more parameters are introduced $\left(T_{d}\right.$ and $N$ ), additional requirements could be considered for the design. A very important issue to be taken into account when considering other controllers is that the filtering properties of $G_{o l}$ must be enough to kept the DF technique valid for predicting the limit cycle oscillation. This study can be done according to the procedure presented in Section 3.4 to validate the design of the PI.

The procedure is summarized in a flowchart which can be found in Figure C.1 in Appendix C.2. Note that once the pair $\left(K_{p}, T_{i}\right)$ to fulfill the requirements on $\gamma_{c g}$ is obtained, the remaining robustness measurements are tested and the controller gain is corrected if needed. Each margin is calculated, and if they are lower than the requirement, $K_{p}$ is reduced according to a detuning factor $k$ that is calculated with the pertinent equations.

In order to show the validity of the proposed tuning methodology the following example is introduced.

Example 5. Consider a system whose transfer function is defined by:

$$
G(s)=\frac{1}{(s+1)^{4}} .
$$


The peak-to-peak amplitude of the measurement noise has a magnitude of $e_{n_{p-p}}=0.03$ and the maximum admissible steady-state error is $e_{s s}=0.1$.

With the aim of avoiding burst of events introduced by an insufficient hysteresis in the sampler due to the noise, the hysteresis $h$ must be slightly greater than the peak-to-peak amplitude of the noise, thus a value of $h=0.04$ is chosen. The upper bound of $\delta$, once $h$ has been chosen, is calculated with the expression of the $e_{s s}$ in $R Q H$ loops, $\delta=2 e_{s s}-h$. A value of $\delta=0.16$ is taken, which is the upper bound, in order to keep the quotient $h / \delta$ low enough to reduce the number of events and to minimize the effect of the margins $\gamma_{h / \delta}$ and $\Phi_{h / \delta}$ in the controller design.

In order to obtain a proper closed-loop response and enough robustness against limit cycles induced by the sampler, the controller is designed to meet the following constraints:

$$
\begin{gathered}
\gamma_{c g} \geq 6 d B \quad \Phi_{c p} \geq 45^{\circ} \\
\gamma_{h / \delta} \geq 2 d B \quad \Phi_{h / \delta} \geq 15^{\circ}
\end{gathered} .
$$

By applying the proposed tuning procedure the parameters for a PI controller are obtained, being $K_{p}=1.05$ and $T_{i}=2.6$, whose exact robustness margins are:

$$
\begin{array}{cc}
\gamma_{c g}=7.22 d B & \Phi_{c p}=45^{\circ} \\
\gamma_{h / \delta}=2.91 d B & \Phi_{h / \delta}=18.6^{\circ}
\end{array}
$$

which fulfill the requirements stated above. The robustness margins to the non-linearity can be visualized in Figure 3.4, where phase and gain margins to the non-linearity have been represented in Nyquist and Nichols diagrams. Figure 3.5 shows the closed-loop response to step changes in the reference and the disturbance inputs. As expected, neither limit cycle oscillations take place nor unnecessary events are generated due to the noise. The amplitude of the control action bumps is $\delta_{u}=K_{p} \delta=0.168$.

In order to show the effect of limiting $\delta_{u}$ on the design, consider the actuator to admit a maximum control action variation of $\delta_{u_{\max }}=0.05$. Taking into account this additional restriction the new parameters for the controller are $K_{p}=0.312$ and $T_{i}=1.037$ and the robustness margins:

$$
\begin{array}{cc}
\gamma_{c g}=9.5126 d B & \Phi_{c p}=45^{\circ} \\
\gamma_{h / \delta}=4.486 d B & \Phi_{h / \delta}=20.95^{\circ}
\end{array} .
$$

The time response of the controlled system is shown in Figure 3.6. As it can be seen, the value of $\delta_{u}$ is limited to 0.05 and consequently a smoother control action is achieved.

In most cases, bigger ratios of $h / \delta$ lead to more restrictive robustness constraints to design the controllers. This fact could induce the idea that those PI designed for ratios $h / \delta$ bigger than the one to be actually implemented in the system always fulfill all the robustness margins and consequentially the most conservative designs are achieved for the SSOD sampler. The following example shows a case that refutes this assumption. 

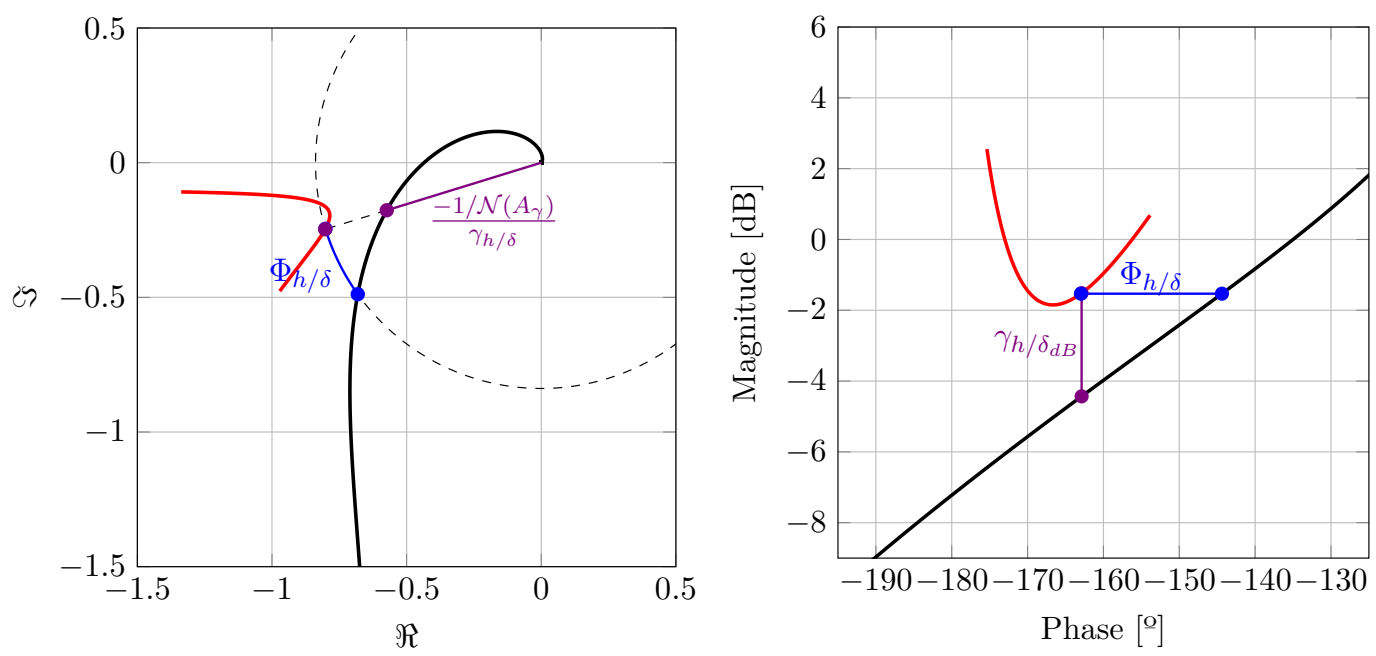

Figure 3.4: Graphical representation of the gain and phase margins to the non-linearity $\left(\gamma_{h / \delta}, \Phi_{h / \delta}\right)$ in Nyquist (left) and Nichols (right) diagrams for the PI controller obtained in Example 5.
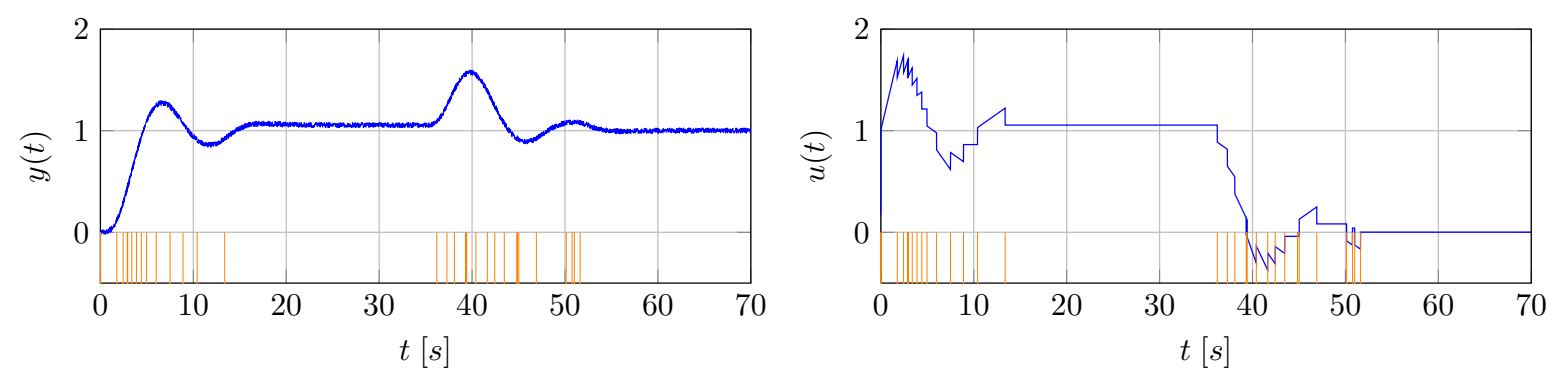

Figure 3.5: Temporal response of the controlled output and control action to unitary step-like changes in reference and disturbance of the example system with the proposed controller and sampler.

Example 6. Consider a system whose transfer function is modeled by:

$$
G(s)=\frac{e^{-5 s}}{(s+1)^{3}} .
$$

For this example consider that the tuning requirements to be met are:

$$
\begin{gathered}
\gamma_{c g} \geq 6 d B \quad \Phi_{c p} \geq 45^{\circ} \\
\gamma_{h / \delta} \geq 4 d B \quad \Phi_{h / \delta} \geq 30^{\circ}
\end{gathered} .
$$

Assume that by applying the procedure to select $\delta$ and $h$ taking into account the noise and $e_{s s}$, the required sampler has a ratio $h / \delta=1 / 20$. However, according to the misconception explained before, in order to have a more robust controller, it is decided to apply the tuning algorithm considering a sampler with ratio $h / \delta=1$, i.e. the $S S O D$ sampler. 

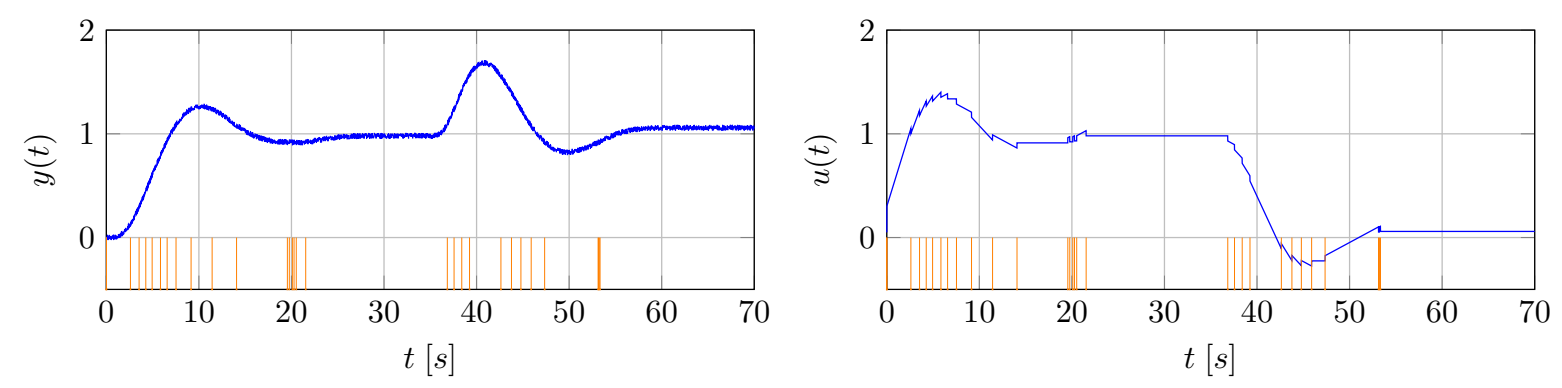

Figure 3.6: Temporal response of the controlled output and control action to unitary step-like changes in reference and disturbance of the example system considering a limitation on the actuator $\delta_{u}=0.05$.
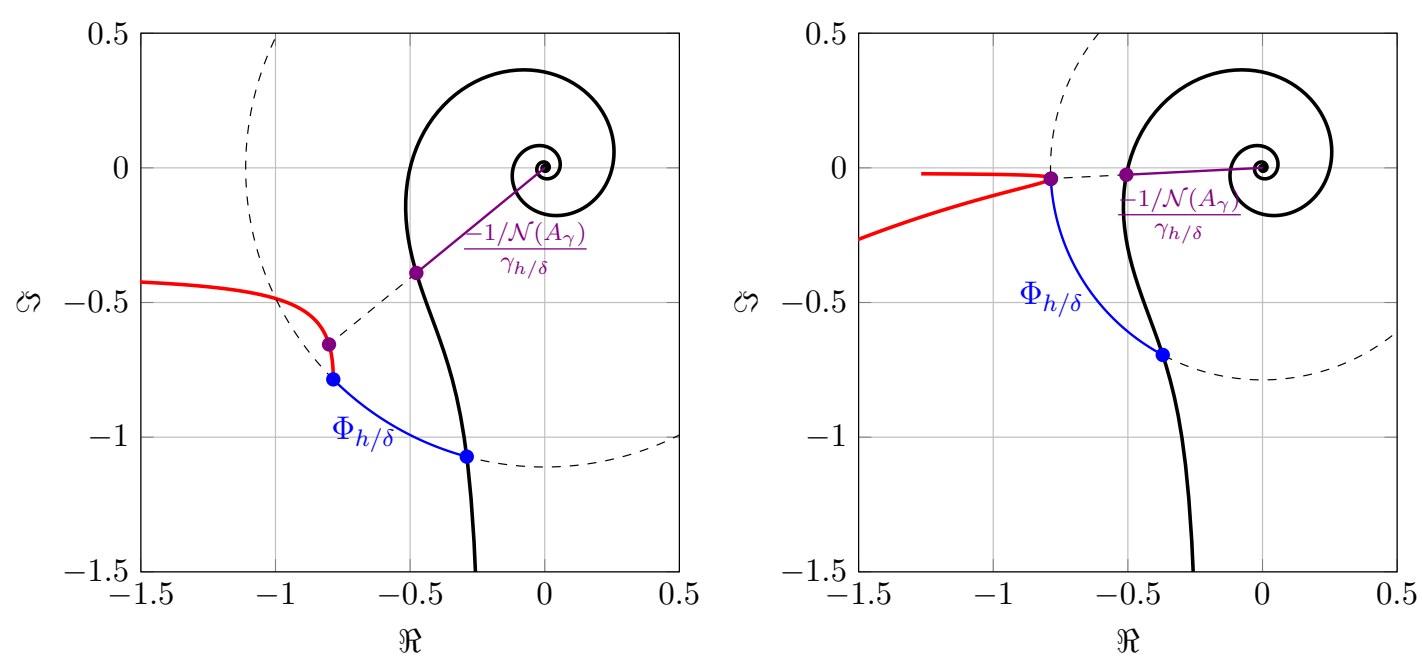

Figure 3.7: Gain and phase margins to the non-linearity $\left(\gamma_{h / \delta}, \Phi_{h / \delta}\right)$ obtained in Example 6 for SSOD sampler (left) and sampler with ratio $h / \delta=1 / 20$ (right).

The resulting PI has $K_{p}=0.518, T_{i}=5.584$ and classical gain and phase margins $\gamma_{c g}=$ $6.02 d B$ and $\Phi_{c p}=72.1^{\circ}$. Referring to the robustness against limit cycles, for the SSOD sampler the margins to the non-linearity are $\gamma_{h / \delta}=4.5072 \mathrm{~dB}$ and $\Phi_{h / \delta}=30^{\circ}$, fulfilling the requirements. However, the margins for the sampler with ratio $h / \delta=1 / 20$ are $\gamma_{h / \delta}=3.8261 \mathrm{~dB}$ and $\Phi_{h / \delta}=59^{\circ}$. As it can be noted, the PI designed for SSOD does not fulfill the robustness condition when used with the RQH sampler: in this case $\gamma_{h / \delta}$ is reduced from $4.5072 d B$ to $3.8261 d B$.

A graphical representation of the margins to the non-linearity is presented in Figure 3.7 where it can be easily seen the effect of reducing the hysteresis on the margins. In this case, the phase margin to the non-linearity increases as expected because of the folding of the DF traces, however, the gain margin to the non-linearity worsens to the point of not fulfilling the tuning requirements, which is also due to the folding of the DF traces over the real axis.

From the precedent example it can be deduced that the controller needed to fulfill the 

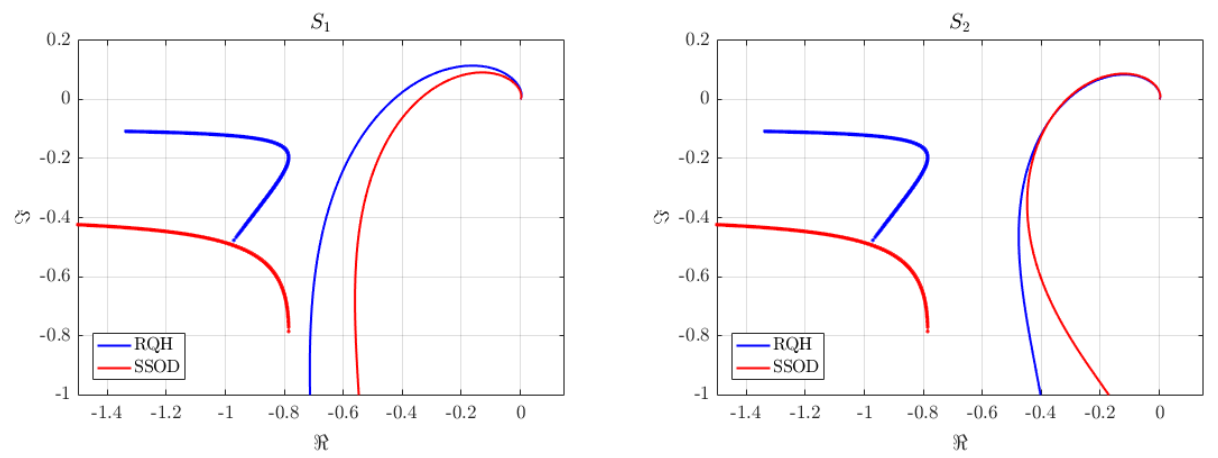

Figure 3.8: Nyquist plot of $G_{o l}$ with the obtained controllers and the inverse negative of the DF for both scenarios.

requirements must be changed if the sampling strategy varies. The following example presents a comparison between the controllers obtained with the proposed method for the specific cases of SSOD and RQH.

Example 7. Consider the scenario presented in Example 5, i.e. same process, noise peak-topeak amplitude and maximum steady-state error. The choice of the RQH sampler will be kept too.

There is a range of the parameter $\delta$ for the SSOD sampler to fulfill the noise and error requirements. This range is defined by the same equations than for the $R Q H$ but having $h=\delta$, resulting in:

$$
e_{n_{p-p}}<\delta<e_{s s}
$$

As choosing a value of $\delta$ close to $e_{n_{p-p}}$ will result in a high event generation rate, a value $\delta=0.1$ will be chosen, i.e. at the limit of the admissible maximum steady-state error.

We will consider two sets of robustness requirements to illustrate different situations. These sets denoted by $S_{1}$ and $S_{2}$ present the following robustness requirements:

$$
\begin{aligned}
& S_{1}: \quad \gamma_{c g} \geq 6 d B \quad \Phi_{c p} \geq 45^{\circ} \quad S_{2}: \quad \gamma_{c g} \geq 6 d B \quad \Phi_{c p} \geq 45^{\circ} \\
& \gamma_{h / \delta} \geq 3 d B \quad \Phi_{h / \delta} \geq 15^{\circ} \quad \gamma_{h / \delta} \geq 3 d B \quad \Phi_{h / \delta} \geq 45^{\circ}
\end{aligned}
$$

$S_{2}$ presents higher restrictions than $S_{1}$ in terms of the phase margin to the non-linearity, therefore, the robustness against limit cycle oscillations will be higher.

Applying the proposed tuning procedure for each set of restrictions and for each sampling strategy four controllers are obtained, whose parameters are gathered in Table 3.1. The open-loop transfer function for both cases under the specified scenarios can be observed in Figure 3.8.

To asses the performance of the controllers several measures will be defined. Firstly, the integral of the absolute sampled error, i.e. at the output of the sampler, as a consequence of a unitary step-like change in the reference (IA $\left.\bar{E}_{r e f}\right)$ and disturbance (IA $\left.\bar{E}_{d}\right)$ inputs. It is defined as:

$$
I A \bar{E}=\int_{0}^{\infty}|\bar{e}(t)| d t
$$



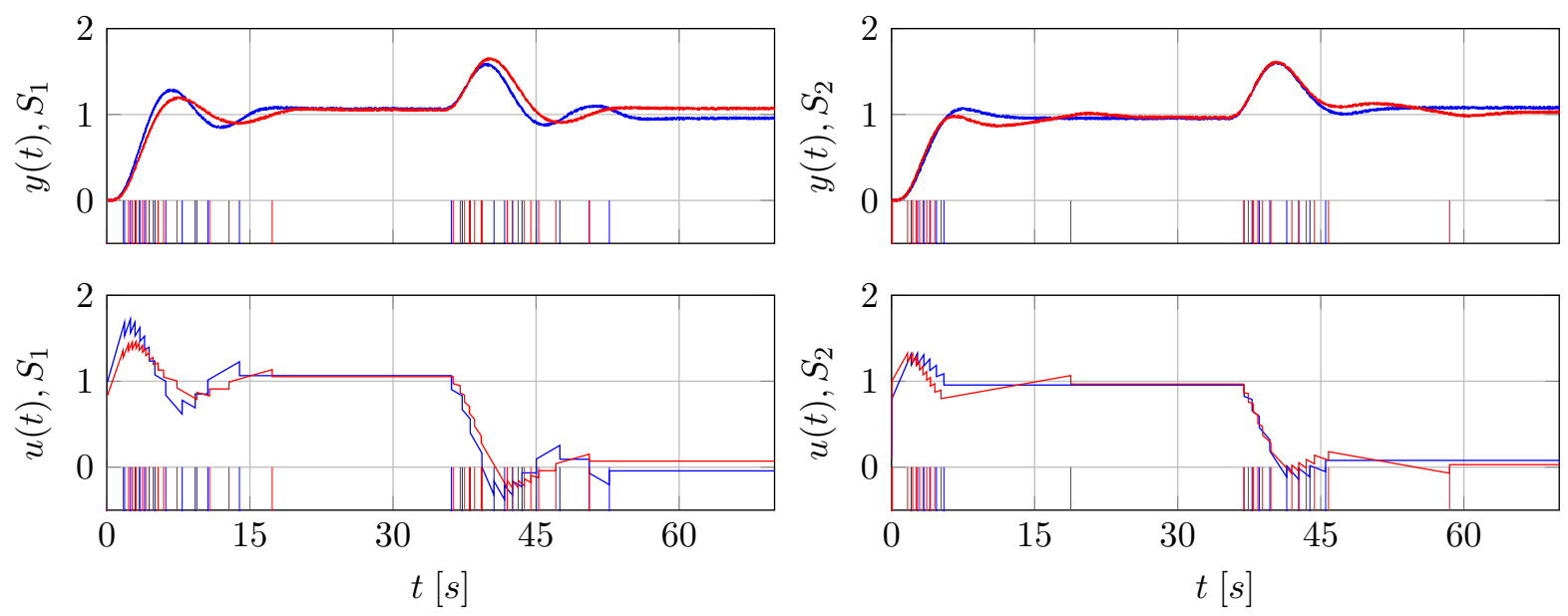

Figure 3.9: Temporal response of the measured output of the process with the controllers obtained in example 7 (blue: RQH and red: SSOD), the events generated and their respective control actions to unitary step changes in the reference (at $t=0 \mathrm{~s}$ ) and disturbance (at $t=35 \mathrm{~s}$ ) inputs.

\begin{tabular}{c|c|ccccccc}
\multicolumn{2}{l|}{} & $\mathbf{K}_{\mathbf{p}}$ & $\mathbf{T}_{\mathbf{i}}$ & $\mathbf{I A E}_{\mathbf{r e f}}$ & $\mathbf{t}_{\mathbf{s}, \text { ref }}$ & $\mathbf{I A E}_{\mathbf{d}}$ & $\mathbf{t}_{\mathbf{s}, \mathbf{d}}$ & $\mathbf{n}_{\text {ev }}$ \\
\hline \multirow{2}{*}{$\mathbf{S}_{\mathbf{1}}$} & $\mathbf{R Q H}$ & 1.01 & 2.5 & 4.545 & 13.899 & 3.538 & 17.678 & 26 \\
& SSOD & 0.817 & 2.57 & 4.701 & 17.334 & 3.795 & 15.578 & 34 \\
\hline \multirow{2}{*}{$\mathbf{S}_{\mathbf{2}}$} & $\mathbf{R Q H}$ & 0.822 & 3.081 & 3.582 & 5.488 & 3.285 & 10.508 & 16 \\
& SSOD & 0.997 & 5.049 & 4.895 & 18.794 & 4.747 & 23.498 & 26
\end{tabular}

Table 3.1: Controllers and performance indexes for each controller and sampler under restrictions $S_{1}$ and $S_{2}$.

being $t=0$ s the point in time where the excitation occurs. In addition, as the classical settling time cannot really be applied due to the appearance of $e_{s s}$, therefore, the settling time used is defined as the elapsed time from the excitation application to the time at witch the system response enters and remains within the final detection thresholds. Finally, the number of triggered events are also used as a measure.

The temporal responses of the four controllers against step-like changes at the reference and disturbance inputs are presented in Figure 3.9, where the RQH case is represented in blue and the SSOD in red.

Considering the case presented by the set of conditions $S_{1}$, it can be seen that the temporal responses of both controllers and samplers do not differ significantly. RQH sampling presents bigger changes in the control action produced by the quantification $\delta$ used, which leads to a slightly more oscillatory response with a higher overshoot. In Table 3.1 the performance measures described above are presented, revealing that the usage of $R Q H$ sampling entails a reduction on the number of events needed to control the system, and according to the obtained performance indexes, this reduction of events does not degrade the performance of the system.

However, in the set of restrictions $S_{2}$, it can be seen a significant improvement of $R Q H$ 


\begin{tabular}{c|ccccccc} 
& $\mathbf{K}_{\mathbf{p}}$ & $\mathbf{T}_{\mathbf{i}}$ & $\mathbf{I A} \overline{\mathbf{E}}_{\mathbf{r e f}}$ & $\mathbf{t}_{\mathbf{s}, \mathbf{r e f}}$ & $\mathbf{I A \overline { \mathbf { E } } _ { \mathbf { d } }}$ & $\mathbf{t}_{\mathbf{s}, \mathbf{d}}$ & $\mathbf{n}_{\mathbf{e v}}$ \\
\hline Proposed & 0.6646 & 2.1206 & 4.8637 & 14.878 & 4.1017 & 13.798 & 41 \\
AMIGO & 0.2473 & 1.8807 & 7.4872 & 18.088 & 7.7062 & 22.048 & 31 \\
One-Third & 0.3202 & 2.8453 & 8.6168 & 24.088 & 8.6736 & 27.388 & 31 \\
Ziegler-Nichols & 0.9257 & 6.0792 & 6.3643 & 30.268 & 6.3643 & 34.768 & 43
\end{tabular}

Table 3.2: Obtained controller and performance parameters for each tuning method.

sampling with regard to SSOD. As expected, the number of events generated is lower for $R Q H$, but in addition, the rest of performance indexes are improved. This is due to the slowness of the SSOD controller, resulting from the required robustness against limit cycle oscillations, which produces a slow response that worsens the performance.

In summary, this example shows how by choosing RQH sampling a robust controller against limit cycle oscillations induced by the sampler can be tunned without degrading significantly the overall performance of the system. An opposed situation is found with SSOD sampling, in which to attain similar levels of robustness than in RQH the controller is forced to present a slower response that degrades the system performance. Additionally, the number of events needed for control is lower for RQH than for SSOD regardless of the robustness to attain.

To highlight the importance of the proposed tuning method over other existing tuning rules the following example is introduced:

Example 8. Consider the process with transfer function:

$$
G(s)=\frac{1-s}{(s+1)^{3}} .
$$

The peak-to-peak amplitude of the measurement noise has been measured to be of 0.04 units and it is required that the response does not present a steady-state error greater than 0.075. With these requirements a RQH sampler is chosen with $h=0.05$ to avoid burst of events due to the noise and $\delta=0.1$ to not surpass the maximum $e_{s s}$ admissible.

A comparative test will be conducted between the performance offered by the proposed tuning method and other well-known methods, namely, AMIGO [4], One-Third [27] and Ziegler-Nichols [79] tuning rules. For the proposed tuning method, a controller will be tunned to meet the following constrains:

$$
\begin{gathered}
\gamma_{c g} \geq 6 d B \quad \Phi_{c p} \geq 45^{\circ} \\
\gamma_{h / \delta} \geq 2 d B \quad \Phi_{h / \delta} \geq 15^{\circ}
\end{gathered} .
$$

For the other tuning rules the temporal response of the process has been approximated by a FOPTD model and their parameters have been obtained. The obtained parameters can be seen in Table 3.2. Needless to say, the controllers tuned with tuning rules other than the proposed in this chapter are not supposed to avoid the intersection with the inverse negative of the DF, and therefore, avoid limit cycle oscillations. In this case, all controllers avoid intersection with the DF traces, indeed, the classical tuning rules present higher robustness measures than the 


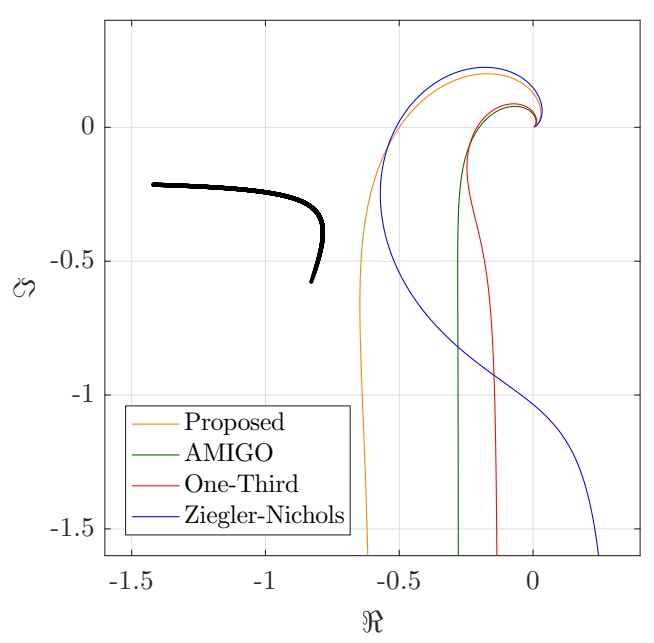

Figure 3.10: Inverse negative of the sampler's DF in this example and the $G_{o l}(j \omega)$ of the four studied controllers and process.

proposed method, which can be appreciate in Figure 3.10 where the open-loop transfer functions of the four controllers and process in the Nyquist diagram are presented as well as the inverse negative of the DF of the sampler under study.

The temporal response of the controlled output can be found in Figure 3.11 as well as the generated events and the control action. In order of appearance: the proposed controller (orange), AMIGO (green), One-Third (red) and Ziegler-Nichols (blue) tuning rules. As it can be seen, all the controllers present enough robustness to avoid limit cycle oscillations. AMIGO and One-Third tuning rules present controllers with a slow temporal response compared to the other rules, however, this is a normal behavior since they also present the highest robustness. The other controllers present a faster response, nevertheless, Ziegler-Nichols struggles to stabilize more than the proposed method.

The measures presented in Example 7 have been used to evaluate the performance of the controllers under study, and have been summarized in Table 3.2. As it can be seen, the proposed tuning rule presents the fastest results, being its integral errors and settling times, both under reference and disturbance changes, the ones with lower values. However, it presents a greater generation event rate, as Ziegler-Nichols, when compared with AMIGO and One-Third tuning rules. This is due to the overdamped temporal response of these last two methods, which matches with its increased robustness and slowness in the response.

\subsection{Design validation}

The gain and phase margins to the non-linearity used in the tuning procedure were obtained using the DF technique, which assumes that the filtering capabilities of the linear part of the 

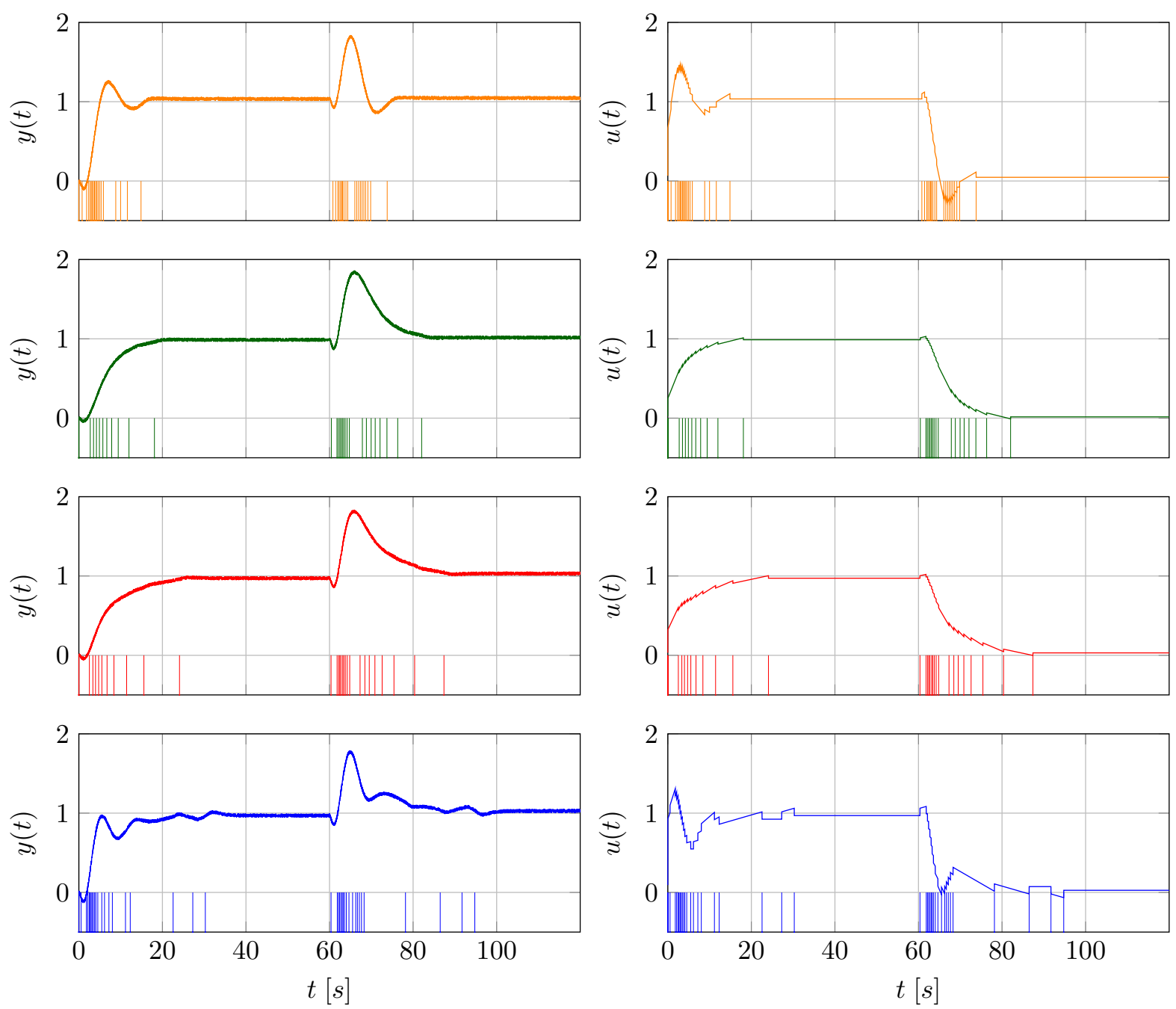

Figure 3.11: Temporal response of the measured output of the process with different controllers, the events generated and their respective control action to unitary step changes in the reference (at $t=0 \mathrm{~s}$ ) and disturbance (at $t=60 \mathrm{~s}$ ) inputs. (Orange: proposed controller. Green: AMIGO. Red:One-Third. Blue: Ziegler-Nichols) 


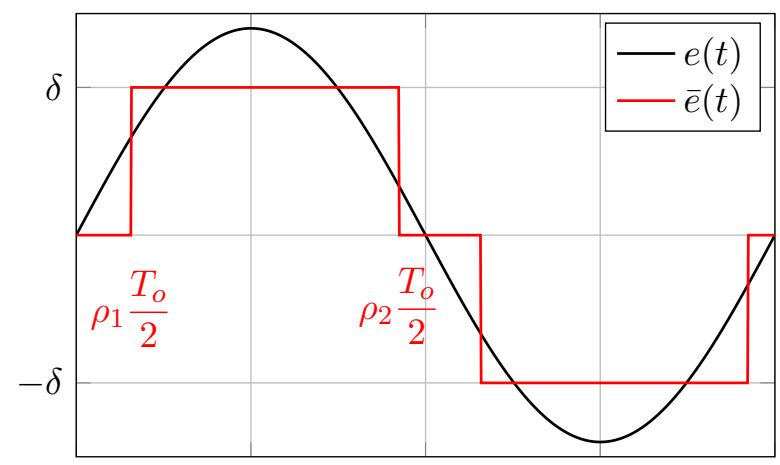

Figure 3.12: Sinusoidal signal and its sampled output with a sampler with a given ratio $h / \delta$.

system are good enough to neglect the high order harmonics effect at the input of the nonlinearity. If this assumption is not fulfilled, the DF viability for predicting limit cycle can conduct to misleading results. In this section, the effect of high order harmonics on the proposed margins is analyzed by studying the variation of the critical points to which each margin is measured. Therefore, the results presented in this section can be used to validate the design procedure presented before or any other tuning method that uses $\gamma_{h / \delta}$ and $\Phi_{h / \delta}$ to check the robustness against limit cycles induced by the RQH sampler.

Consider the input to the non-linearity to be a sine wave with period $T_{o}$ and amplitude $A$, which is considered high enough to produce single-leveled symmetric oscillations $(m=1)$ on the sampled signal $\bar{e}(t)$ as it is shown in Figure 3.12. The switches on the sampled signal $\bar{e}(t)$ are produced at the time fractions $\rho_{1}$ and $\rho_{2}$. In those switching points the value of the error signal $e(t)$ is:

$$
e\left(\rho_{p} \frac{T_{o}}{2}\right)=\left\{\begin{array}{cc}
\delta\left(\frac{1}{2}+\frac{h}{2 \delta}\right) & \text { for } p=1 \\
\delta\left(\frac{1}{2}-\frac{h}{2 \delta}\right) & \text { for } p=2
\end{array} .\right.
$$

As a sinusoidal signal has been supposed at the input of the non-linearity, the value of the time fractions $\rho_{1}$ and $\rho_{2}$ can be obtained:

$$
\begin{aligned}
& \rho_{1}=\frac{1}{\pi} \arcsin \left(\frac{\delta}{A}\left(\frac{1}{2}+\frac{h}{2 \delta}\right)\right) \\
& \rho_{2}=1-\frac{1}{\pi} \arcsin \left(\frac{\delta}{A}\left(\frac{1}{2}-\frac{h}{2 \delta}\right)\right) .
\end{aligned}
$$

Expressing the sampled signal $\bar{e}(t)$ as a Fourier series, and operating with the obtained expression of this signal through the loop, considering $y_{r}(t)=0$, and thus, $e(t)=-y(t)$, it can 
be obtained the resulting expression of $e(t)$, in which, evaluating at $t=\rho_{1} \frac{T_{o}}{2}$ and $t=\rho_{2} \frac{T_{o}}{2}$ :

$e\left(\rho_{1} \frac{T_{o}}{2}\right)=-\frac{4 \delta}{\pi} \sum_{n_{\text {odd }}}^{\infty} \frac{1}{n}\left[\Re\left\{G_{o l}\left(j n \omega_{o}\right)\right\} \frac{1}{2} \sin \left(n \pi\left(\rho_{2}-\rho_{1}\right)\right)+\Im\left\{G_{o l}\left(j n \omega_{o}\right)\right\} \sin ^{2}\left(\frac{n \pi}{2}\left(\rho_{2}-\rho_{1}\right)\right)\right]$,

$e\left(\rho_{2} \frac{T_{o}}{2}\right)=-\frac{4 \delta}{\pi} \sum_{n_{\text {odd }}}^{\infty} \frac{1}{n}\left[\Re\left\{G_{\text {ol }}\left(j n \omega_{o}\right)\right\} \frac{1}{2} \sin \left(n \pi\left(\rho_{2}-\rho_{1}\right)\right)-\Im\left\{G_{o l}\left(j n \omega_{o}\right)\right\} \sin ^{2}\left(\frac{n \pi}{2}\left(\rho_{2}-\rho_{1}\right)\right)\right] ;$

substituting these values of $e(t)$ in the switching points presented in equation (3.24), this equation can be expressed otherwise as:

$$
\begin{aligned}
\Re\left\{G_{o l}\left(j \omega_{o}\right)\right\} & =-\frac{\frac{\pi}{4}+\sum_{n=3,5, \ldots}^{\infty} \frac{1}{n} \Re\left\{G_{o l}\left(j n \omega_{o}\right)\right\} \sin \left(n \pi\left(\rho_{2}-\rho_{1}\right)\right)}{\sin \left(\pi\left(\rho_{2}-\rho_{1}\right)\right)}, \\
\Im\left\{G_{o l}\left(j \omega_{o}\right)\right\} & =-\frac{\frac{\pi h}{8 \delta}+\sum_{n=3,5, \ldots}^{\infty} \frac{1}{n} \Im\left\{G_{o l}\left(j n \omega_{o}\right)\right\} \sin ^{2}\left(\frac{n \pi}{2}\left(\rho_{2}-\rho_{1}\right)\right)}{\sin ^{2}\left(\frac{\pi}{2}\left(\rho_{2}-\rho_{1}\right)\right)} .
\end{aligned}
$$

Replacing the expressions of $\rho_{1}$ and $\rho_{2}$ which were obtained in equation (3.25), the previous expressions can be rewritten as:

$$
\begin{aligned}
\Re\left\{G_{o l}\left(j \omega_{o}\right)\right\} & =-\frac{\frac{\pi}{4}+\sum_{n=3,5, \ldots}^{\infty} \frac{1}{n} \Re\left\{G_{o l}\left(j n \omega_{o}\right)\right\} \sin (n \theta)}{\sin (\theta)}, \\
\Im\left\{G_{o l}\left(j \omega_{o}\right)\right\} & =-\frac{\frac{\pi h}{4 \delta}+\sum_{n=3,5, \ldots}^{\infty} \frac{1}{n} \Im\left\{G_{o l}\left(j n \omega_{o}\right)\right\}(1+\cos (n \theta))}{1+\cos (\theta)},
\end{aligned}
$$

where:

$$
\theta=\arcsin \left(\frac{\delta}{2 A}\left[\left(1-\frac{h}{\delta}\right) \sqrt{1-\left[\frac{\delta}{2 A}\left(1+\frac{h}{\delta}\right)\right]^{2}}+\left(1+\frac{h}{\delta}\right) \sqrt{1-\left[\frac{\delta}{2 A}\left(1-\frac{h}{\delta}\right)\right]^{2}}\right]\right),
$$

which depends on the non-linearity characteristics.

Equations in (3.26) describe the real and imaginary parts of $G_{o l}\left(j \omega_{o}\right)$ when the system presents oscillation of frequency $\omega_{o}$. As it can be seen, these expressions depend on the high order harmonics of the oscillation frequency: $n \omega_{o}, n=3,5, \ldots$, which are neglected in the DF approach. Therefore, these equations can be used to validate the controllers obtained by the tuning method proposed in section 3.3. To do that, more accurate estimations of the points with respect to which the margins $\gamma_{h / \delta}$ and $\Phi_{h / \delta}$ are measured can be obtained by using equations in (3.26) with a reasonable number of harmonics and the values of $G_{o l}\left(j n \omega_{o}\right), n=3,5, \cdots$ approximated as:

$$
G_{o l}\left(j n \omega_{o}\right)=G_{o l}\left(j n \omega_{\gamma}\right) \gamma_{h / \delta} ; \quad n=3,5, \cdots
$$


to evaluate the gain margin accuracy, and:

$$
G_{o l}\left(j n \omega_{o}\right)=G_{o l}\left(j \omega_{\Phi}\right) \cdot 1 \not-\Phi_{h / \delta} ; \quad n=3,5, \cdots
$$

to evaluate the phase margin. In fact, equations (3.27) and (3.28) provide the values $G_{o l}$ in the higher order harmonics of frequencies where the oscillations will take place if the gain or phase lag of $G_{o l}$ increase on $\gamma_{h / \delta}$ or $\Phi_{h / \delta}$ respectively.

Hence, to validate the gain and phase margins to the non-linearity obtained, and therefore, the controller that has been tuned, the point obtained using equations (3.26) and (3.27), which will be denoted by $G_{o l, \gamma}\left(j \omega_{o}\right)$, has to be compared to the point $-1 / \mathcal{N}\left(A_{\gamma}\right)$. Similarly, the point obtained with equations (3.26) and (3.28), which will be referred to as $G_{o l, \Phi}\left(j \omega_{o}\right)$, has to be compared to the point $-1 / \mathcal{N}\left(A_{\Phi}\right)$.

To show the variation of these critical points with respect to those where the gain and phase margin to the non-linearity are measured and how this variation is used to validate the proposed controllers, the following example is introduced.

Example 9. Consider a system whose transfer function is defined by:

$$
G(s)=\frac{1}{(s+1)^{4}} .
$$

A PI controller has been tuned applying AMIGO method [4] by approximating this system by a FOPTD model, obtaining $K_{p}=0.301$ and $T_{i}=2.4268$. Consider in this case that the system is placed in a loop which contains a sampler with ratio $h / \delta=2 / 3$. The robustness margins to the non-linearity have been measured and result in $\gamma_{h / \delta}=11.51 \mathrm{~dB}$ and $\Phi_{h / \delta}=39.61^{\circ}$. The gain margin has been obtained at a frequency $\omega_{\gamma}=0.4703 \mathrm{rad} / \mathrm{s}$ and with a ratio $\delta / A=0.834$, while the phase margin has been obtained at a frequency $\omega_{\Phi}=0.1218 \mathrm{rad} / \mathrm{s}$ and with a ratio $\delta / A=0.833$.

The points where these measures have been obtained can be seen graphically in Figure 3.13, where it has been represented the negative inverse of the $D F$ and $G_{o l}(j \omega)$ in solid black line and the measured robustness margins with a circle marker in blue for the gain margin and in red for the phase margin, in the Nyquist and Nichols diagrams.

Then, to test the validity of the margins, equations in (3.26) have been used considering the following 3 harmonics: $n=3,5$ and $\%$. In those equations, the open-loop transfer function to test is the one presented in equation (3.27) to test the gain margin and (3.28) to test the phase margin.

As a result, the new location of the critical points is obtained. These new points have been represented with a cross of their respective color in Figure 3.13, and the respective new robustness margins have been measured to these points. In this case, the variation between $-1 / \mathcal{N}\left(A_{\gamma}\right)$ and $G_{o l, \gamma}\left(j \omega_{o}\right)$ and between $-1 / \mathcal{N}\left(A_{\Phi}\right)$ and $G_{o l, \Phi}\left(j \omega_{o}\right)$ is not very important. Thus, as the variation between the robustness margins is very slight, being both obtained margins more restrictive than 

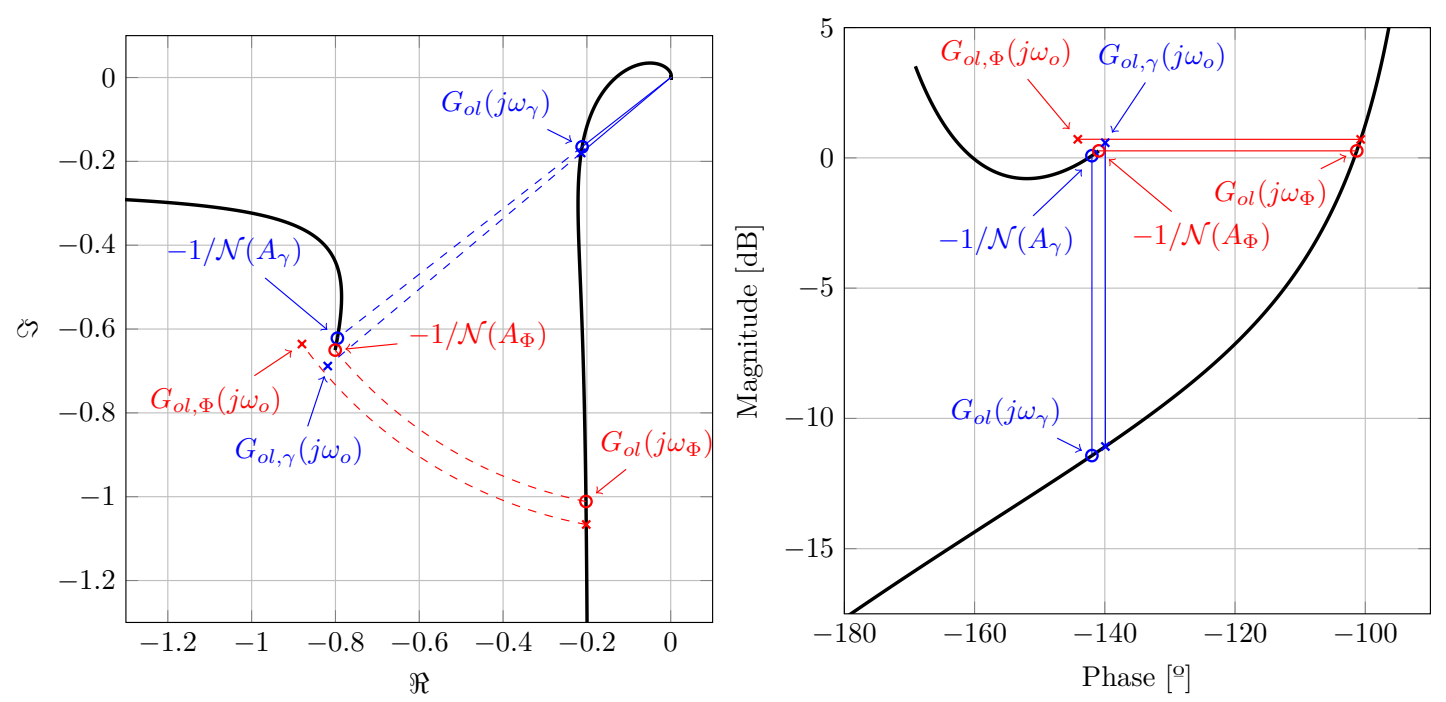

Figure 3.13: Harmonic analysis of the validity of the proposed margins for $G_{o l}(s)$ and the sampler with ratio $h / \delta=2 / 3$.

the corrected margins considering the high order harmonic contribution, the obtained controller can operate safely in this loop.

Nevertheless, the corrected margins are not always beneficial in terms of robustness. For example, consider now that the sampler used above is replaced by another sampler with ratio $h / \delta=1 / 6$. As in the precedent case, the robustness measures to the non-linearity have been obtained at the critical frequencies and $\delta / A$ ratios. Using equations (3.26), (3.27) and (3.28) as it has been explained before, the new location of the critical points has been obtained. In Figure 3.14 it has been represented the negative inverse of the DF traces, the transfer function and the critical points obtained with the DF and with the harmonic analysis calculus. Here, it can be seen that the variation of the critical points implies a reduction in the effective margins, being this reduction more visible in the case of the phase margin.

In this case, the variation in the margins is not significant enough to induce limit cycle oscillations in the temporal response, in fact, considering the new recalculated margins, the controller still provides a considerable degree of robustness to the system. However, this proves that the influence of high order harmonics is not always beneficial in terms of robustness and that the validation of the controller design should be carried out, specially when systems with low filtering capabilities are involved, because the DF approach cannot be applicable with a high reliability.

In general lines, the proposed margins give an accurate order of magnitude of the proximity to the oscillations due to the effect of the non-linearity. As it has been seen in the precedent example, the effect of the high order harmonics takes an important role in the accuracy of the robustness margins to the non-linearity, being the variation of the critical points beneficial or detrimental depending on the case. 

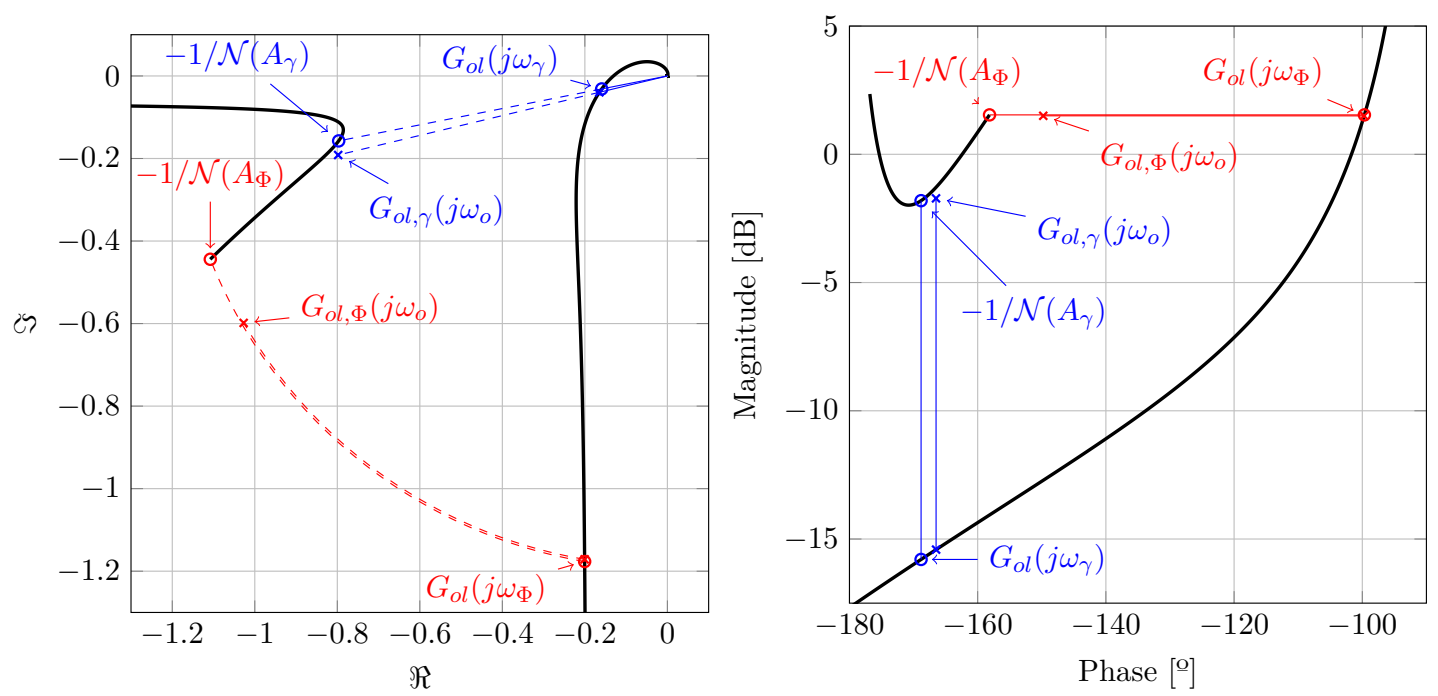

Figure 3.14: Harmonic analysis of the validity of the proposed margins for a given system and sampler.

In particular, in those cases where $\Phi_{h / \delta}$ is obtained for relative low values of $\omega_{\Phi}$, the variation of the critical point of the phase margin produced by the harmonic analysis is more remarkable, resulting in decreasing the effective phase margin to the non-linearity. In those cases, despite the variation on the phase margin, the corrected phase margin measure still provides enough robustness.

\subsection{Conclusion}

In this chapter, a tuning procedure for PI controllers within a loop with a RQH sampling strategy has been provided. Prior to the controller tuning, the parameters that define the RQH sampler must be chosen. Guidelines to select the proper parameters for a system from specifications have been provided.

The tuning procedure consists of 3 steps in which both, the classical robustness margins and the robustness margins to the limit cycles produced by the sampler proposed in this chapter, have been taken into account. By considering both, not only the limit cycle oscillations induced by the sampler are avoided, but also a proper closed-loop temporal response is assured.

Several simulation examples where the proposed tuning method is applied are shown. Those examples illustrate the usefulness of the method and how a loss in robustness can be suffered when the method is not properly used.

Finally, the validation of the controller design has been tested by considering the effect on the robustness measures of higher order harmonics. In the cases where the DF technique can be used, it has been confirmed that the proposed margins to predict the appearance of oscillations are accurate and this validation can be skipped. Nevertheless, in those cases where systems with 
low filtering capabilities are involved the validation of the controller designed is recommendable due to the high order harmonic influence on the robustness. 


\title{
Analysis and tuning of SSOD and RQH systems with Tsypkin method
}

\begin{abstract}
A procedure for tuning PID controllers with SSOD sampling for FOPTD systems is proposed. It is based on the definition of a new robustness measure to avoid limit cycle oscillations, called the Tsypkin margin $\left(M_{T}\right)$. This margin is based on the Tsypkin method and does not rely on the attenuation of high order harmonics, as the describing function approaches require. Therefore, the avoidance of limit cycle oscillations can be guaranteed for any system, as a difference with the describing function based procedures. The procedure allows obtaining the PID controller that minimizes the disturbance IAE while fulfilling constraints on robustness to oscillations and on control action bumps due to the SSOD sampling. A freely available Java tool has been developed in order to simplify the application of the tuning procedure. In case of a non FOPTD system, it first calculates an approximate FOPTD model. This chapter shows that the derivative filter parameter $N$ is a critical tuning parameter in order to find a compromise between performance and control action bumps. The analysis method is then extended to loops with RQH sampling strategy.
\end{abstract}

\subsection{Introduction}

Nowadays Event-Based Controllers are a promising alternative to the classical time driven control systems to reduce the measurement frequency needed for the control without degrading the closed-loop performance. This is a basic requirement for controllers in networked control systems where many devices (sensors, actuators, controllers) share a communication channel with limited bandwidth. The reduction in the number of transmitted messages improves the network overall behavior, for example avoiding dropouts and delays. The use of wireless communications 
in control applications has also encouraged the development of Event-Based Controllers. In this case, the reduction of data transmission implies an important decrease in power consumption, therefore increasing the lifetime of batteries of self-powered remote sensors [24].

The reduction of the measurement frequency in Event-Based Control systems strongly depends on the strategy used to generate the events to send data through the network. In that sense, Send-On-Delta (SOD) strategy has emerged as one of the most promising approaches, which reduces considerably the sending of new data $[19,50]$. The SOD method consists in transmitting data from the sensor to the controller node only if the measurement value changes more than a given specified $\delta$ value, [45]. A particular kind of SOD, named Symetric-Send-On-Delta (SSOD), is proposed in [10]. The SSOD sampler quantifies the input signal by a quantity multiple of a constant value $\delta$, then, a new value of the input is sent by the sampler when the input changes in a quantity $\delta$.

One of the key points in the analysis and design of event-based control systems is the existence of limit cycles that can lead to sustained oscillations in the closed-loop response. The characterization of limit cycles for different kind of systems, such as Integrator processes Plus Time Delay (IPTD), First Order processes Plus Time Delay (FOPTD), and Second Order processes Plus Time Delay (SOPTD), when using a SSOD sampling strategy has been presented in [17]. The study of the limit cycles is important to predict the magnitude and frequency of the oscillation that could appear in a system as a consequence of the detuning of the controller produced by changes in the system's dynamic. From the PID tuning point of view, one of the main goal is to prevent limit cycles in order to avoid undesired oscillations which can reduce the overall performance of the control system or produce excessive actuator wear.

In [60] and [56], tuning methods for PI controllers with SSOD sampler have been developed based on new robustness margins for limit cycles, which were obtained by applying the Describing Function (DF) technique and connect with the classical concepts of phase and gain margins. The same approach was used in [61], where a unified design of SSOD-PID control architecture for self-regulating and integral processes was investigated. The use of the DF led to introduce tools of the classical control theory, as the Nyquist plot, for the analysis and design of Event-Based Control systems. That could make the design of event based PID controllers more understandable and consequently boost its application in industry, since these concepts are generally taught in the automatic control undergraduate courses.

It is well known, however, that the validity of the DF depends on the filtering properties of the open-loop transfer function: only under certain filtering conditions the higher order harmonics can be neglected and the DF can be successfully applied. Therefore, low order models, such as FOPTD and SOPTD, which are commonly used to describe actual industrial processes, are excluded from this approach and new methods are required to use frequency response based strategies. Concerning the kind of controller, the use of PID which increase the bandwidth with respect to the PI case could be an additional cumbersome for the application of the DF. In [43], the authors proposed a new robustness measure to avoid limit cycle on SSOD based PI controllers. The proposal is based on Tsypkin's method [75], which has been widely used to study relay control systems, that is the reason why the robustness measure has been named 
Tsypkin's margin $\left(M_{T}\right)$. Unlike the margins proposed in [60] and [56], which are based on the $\mathrm{DF}$, the robustness margin $M_{T}$ is valid not only for systems with good filtering capabilities but also for systems whose dynamic is described by low order models.

In this chapter, a new method for tuning PID controllers for FOPTD systems using a SSOD sampling strategy is proposed. It is well known that the FOPTD models admit a dimensionless representation that allows expressing some results, including homogeneous tuning rules that depend on the quotient between the time delay and time constant, [7]. Combining this idea with the concept of Tsypkin's margin, a general framework for tuning SSOD based PID controllers is developed. The proposal can be applied to any homogeneous tuning rule in order to evaluate its performance when applied to SSOD based PID. This general framework has been then applied to the tuning method developed by the author in [66] resulting in a set of tuning rules for SSOD-PID which takes into account an optimum trade-off between IAE index, the control action variations due to changes of magnitude $\delta$ on the measured variable, and the robustness to limit cycle expressed in terms of $M_{T}$. The analysis methodology has been then extended to loops with RQH sampling.

The tuning of SSOD based PI controllers for FOPTD systems has been previously addressed in [11], where some rules were designed by minimizing the $1 \%$ settling time of the closedloop response. In this chapter, both PI and PID structures are taken into account, and it is proved that the latter can significantly improve the system performance without degrading the robustness to limit cycles measured in terms of $M_{T}$. Additionally, an extensive study about the effect of the derivative filter coefficient on the closed-loop behavior is also presented here. Finally, the adaptation of $M_{T}$ to RQH sampling schemes [42] to evaluate their robustness against limit cycle is presented.

\subsection{Problem statement}

Consider the networked control system shown in Figure 4.1, where $C(s)$ and $G(s)$ are the controller and the process transfer functions respectively, $y_{r}$ is the reference signal to be tracked, $y$ is the controlled output, and $p$ is the disturbance input. It is supposed that the controller is located near the actuator and the sensor sends measurements of process output $y$ (or more precisely of the tracking error $e$ ) to the controller through a communication network using the SSOD strategy. The $\mathrm{ZOH}$ block keeps in $\bar{e}$ the last sent value of process output $e^{*}$ until a new value is transmitted by the SSOD block. Communication delays through the network are represented by the term $\exp \left(-t_{d} s\right)$.

This control scheme was first proposed in [10] considering $C(s)$ a PI controller, so the authors called it SSOD-PI architecture. As commented in the introduction, some guidelines for PI tuning were given in [11] when the process $G(s)$ is a FOPTD model. The goal in this chapter is to design a PID controller with transfer function given by equation (4.1) in order to reach an adequate performance and robustness of the closed-loop system. The objective of the tuning procedure is not only to guarantee stability but also to avoid persistent oscillations or limit cycles that can appear due to the SSOD strategy if the controller is not designed properly. 


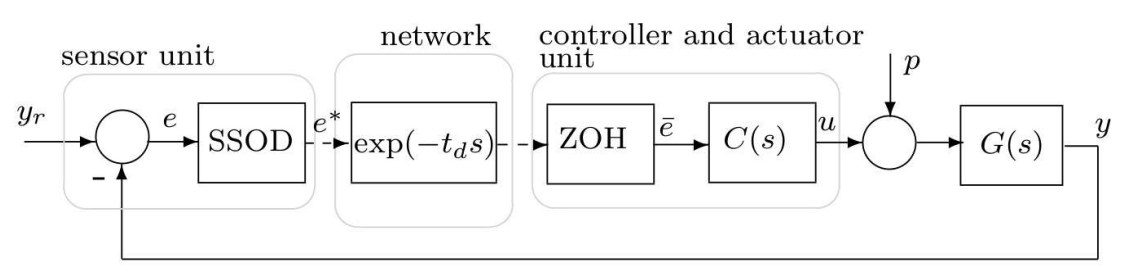

Figure 4.1: Networked control system with SSOD sampling strategy. SSOD- $C(s)$ architecture.

$$
C(s)=K_{p}\left(1+\frac{1}{T_{i} s}+\frac{N T_{d} s}{T_{d} s+N}\right) .
$$

Previous works have addressed the tuning of SSOD-PI controllers by predicting limit cycles using the Describing Function [60, 56], a well known tool used in the analysis of non-linear control systems. In those works, one of the design objectives was to avoid the intersection between the negative inverse of the DF with the open-loop transfer function on the polar plot. Nevertheless, it is known that the DF technique relies on the filtering capabilities of the linear part of the control loop, which must be capable of filtering enough the high frequency harmonics. The linear part involves both the process and the controller dynamics, therefore, for processes with low filtering capabilities and PID controllers, which tend to increase the bandwidth with respect to the PI case, the DF could not be a suitable approach to analyze the existence of limit cycles. This fact is illustrated through the following examples.

Example 10. Consider the FOPTD model whose transfer function is:

$$
G(s)=\frac{e^{-0.2 s}}{s+1}
$$

Controllers $C(s)$ have been tuned according to Ziegler-Nichols [79], Cohen-Coon [18], AMIGO [4] and Sanchis-Romero-Balaguer [66] (SRB for the sake of brevity) methods. The resulting PID parameters are gathered in Table 4.1. The system in Figure 4.1 admits the Hammerstein-Wiener representation shown in Figure 4.2, being SSOD_ZOH the combination of SSOD and ZOH blocks. The condition to avoid limit cycles is

$$
G_{o l}(j \omega) \neq-\frac{1}{\mathcal{N}}, \quad \forall \omega
$$

where $G_{o l}(j \omega)$ is the open-loop transfer function:

$$
G_{o l}(j \omega)=\exp \left(-t_{d} j \omega\right) C(j \omega) G(j \omega)
$$

and $\mathcal{N}$ is the describing function of the SSOD_ZOH block. Graphically this condition implies no intersection between the Nyquist diagram and the negative inverse of $\mathcal{N}$.

Figure 4.3 shows the Nyquist diagrams obtained with the controllers in Table 4.1 and the plot of $-1 / \mathcal{N}$. It can be seen that the open-loop transfer functions corresponding to ZieglerNichols and Cohen-Coon methods intersect with $-1 / \mathcal{N}$, so these systems will present limit cycle 


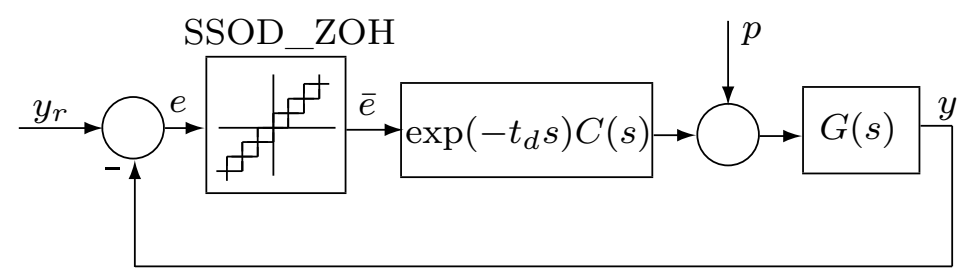

Figure 4.2: Non-linear equivalent system to the control system with SSOD sampling strategy in Figure 4.1.

Table 4.1: PID Parameters with different tuning rules for the system presented in equation (4.2).

\begin{tabular}{c|cccc} 
& $\mathbf{K}_{\mathbf{p}}$ & $\mathbf{T}_{\mathbf{i}}$ & $\mathbf{T}_{\mathbf{d}}$ & $\mathbf{N}$ \\
\hline Ziegler-Nichols & 6 & 0.4 & 0.1 & 10 \\
Cohen-Coon & 6.917 & 0.455 & 0.07 & 10 \\
AMIGO & 2.45 & 0.587 & 0.094 & 10 \\
SRB & 2.181 & 0.484 & 0.115 & 10
\end{tabular}

oscillations. This is not the case for AMIGO and SRB methods, for which intersections between $G_{o l}$ and $-1 / \mathcal{N}$ do not take place, consequently, they are not expected to oscillate. Simulations of the controlled systems presented in Figure 4.4 refute the predictions based on the DF since the four controllers lead to oscillatory behaviors. As it can be seen, each controller induces oscillations with different waveforms because the difference in the tuning parameters produce different $G_{o l}(s)$, each one with its own limit cycle. Additionally, it is worth noticing that the waveforms in Figure 4.4 are, generally speaking, hardly sinusoidal. This is due to the failure of $G_{o l}(s)$ to attenuate higher harmonics sufficiently. It is the waveform's harmonic content which is responsible for the unsuccessful application of the DF in this example.

\subsection{New robustness margin for existence of limit cycles}

\subsubsection{Tsypkin's method approach}

In order to predict more accurately the existence of limit cycles in the control systems presented in Figure 4.1 (or equivalently in Figure 4.2) and avoid them, an approach based on the Tsypkin's method [75] is proposed, which is valid independently of the filtering characteristics of the linear part of the system. Therefore, this approach is especially interesting for low order systems, as for example FOPTD systems that are considered in this chapter.

In systems with a SSOD_ZOH non-linearity as that shown in Figure 4.2, the general shape of an oscillation is depicted in Figure 4.5, in which the half period of an oscillation of $m$ levels is shown. In this figure, the error signal $e$ has been represented, whose slope may be of any magnitude. The sampled error signal $\bar{e}$ has also been plotted as a stair-like signal with step widths defined by $\left(\rho_{p}-\rho_{p-1}\right) T_{o} / 2$, being $T_{o}$ the oscillation period and $\left.\rho_{i} \in\right] 0,1[$. 


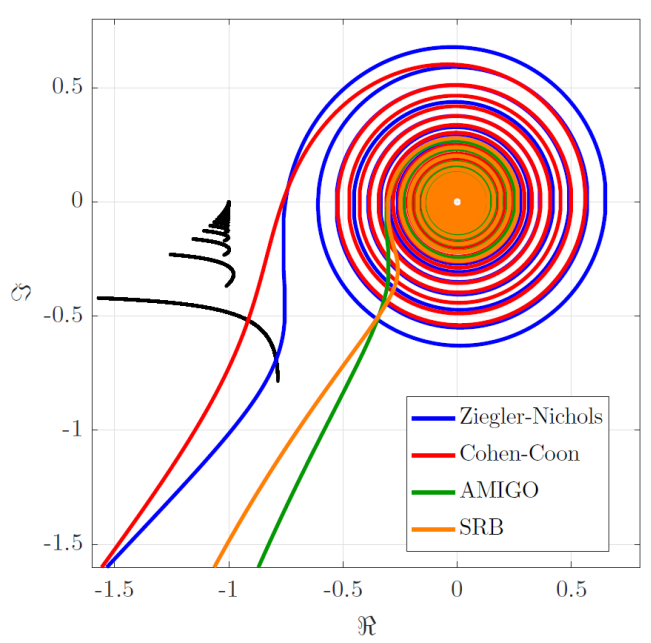

Figure 4.3: Plots of the open-loop transfer functions obtained with the PID parameters in Table 4.1 (colored lines) and $-1 / \mathcal{N}$ (black lines) in the Nyquist diagram.

The Tsypkin's method formalizes the situation represented in Figure 4.5 in the set of mathematic relations that define the oscillation conditions presented in equations (4.3) and (4.4).

$$
\begin{gathered}
E C_{p} \mid e\left(\rho_{p} \frac{T_{o}}{2}\right)= \begin{cases}p \delta & \text { for } p=1,2 \ldots m \\
(2 m-p) \delta & \text { for } p=m+1, \ldots 2 m\end{cases} \\
I C_{p} \mid \frac{d e\left(\rho_{p} \frac{T_{o}}{2}\right)}{d t}= \begin{cases}>0 & \text { for } p=1,2 \ldots m \\
<0 & \text { for } p=m+1, \ldots 2 m\end{cases}
\end{gathered}
$$

These conditions are referred to the values of $e$ and their derivative in specific times $\rho_{p} \frac{T_{o}}{2}$, that is, when the thresholds of magnitude $p \delta$ are crossed. By applying the Fourier series and some basic calculations (see Appendix A) a general expression of $e\left(\rho_{p} \frac{T_{o}}{2}\right)$ can be obtained, equation (4.5), where $n$ is the number of harmonics:

$$
\begin{aligned}
e\left(\rho_{p} \frac{T_{o}}{2}\right)=-\frac{4 \delta}{\pi} \sum_{n=1,3,5, \ldots}^{\infty} \frac{1}{n} \Re\left\{G_{o l}\left(j n \omega_{o}\right)\right\}\left(\sum_{i=1}^{m} i \sin \left(\frac{n \pi}{2}\left(\rho_{i+1}-\rho_{i}\right)\right) \cos \left(n \pi\left(\frac{\rho_{i+1}+\rho_{i}}{2}-\rho_{p}\right)\right)\right. \\
\left.+\sum_{i=m+1}^{2 m-1}(2 m-i) \sin \left(\frac{n \pi}{2}\left(\rho_{i+1}-\rho_{i}\right)\right) \cos \left(n \pi\left(\frac{\rho_{i+1}+\rho_{i}}{2}-\rho_{p}\right)\right)\right) \\
-\frac{4 \delta}{\pi} \sum_{n=1,3,5, \ldots}^{\infty} \frac{1}{n} \Im\left\{G_{o l}\left(j n \omega_{o}\right)\right\}\left(\sum_{i=1}^{m} i \sin \left(\frac{n \pi}{2}\left(\rho_{i+1}-\rho_{i}\right)\right) \sin \left(n \pi\left(\frac{\rho_{i+1}+\rho_{i}}{2}-\rho_{p}\right)\right)\right. \\
\left.+\sum_{i=m+1}^{2 m-1}(2 m-i) \sin \left(\frac{n \pi}{2}\left(\rho_{i+1}-\rho_{i}\right)\right) \sin \left(n \pi\left(\frac{\rho_{i+1}+\rho_{i}}{2}-\rho_{p}\right)\right)\right)
\end{aligned}
$$



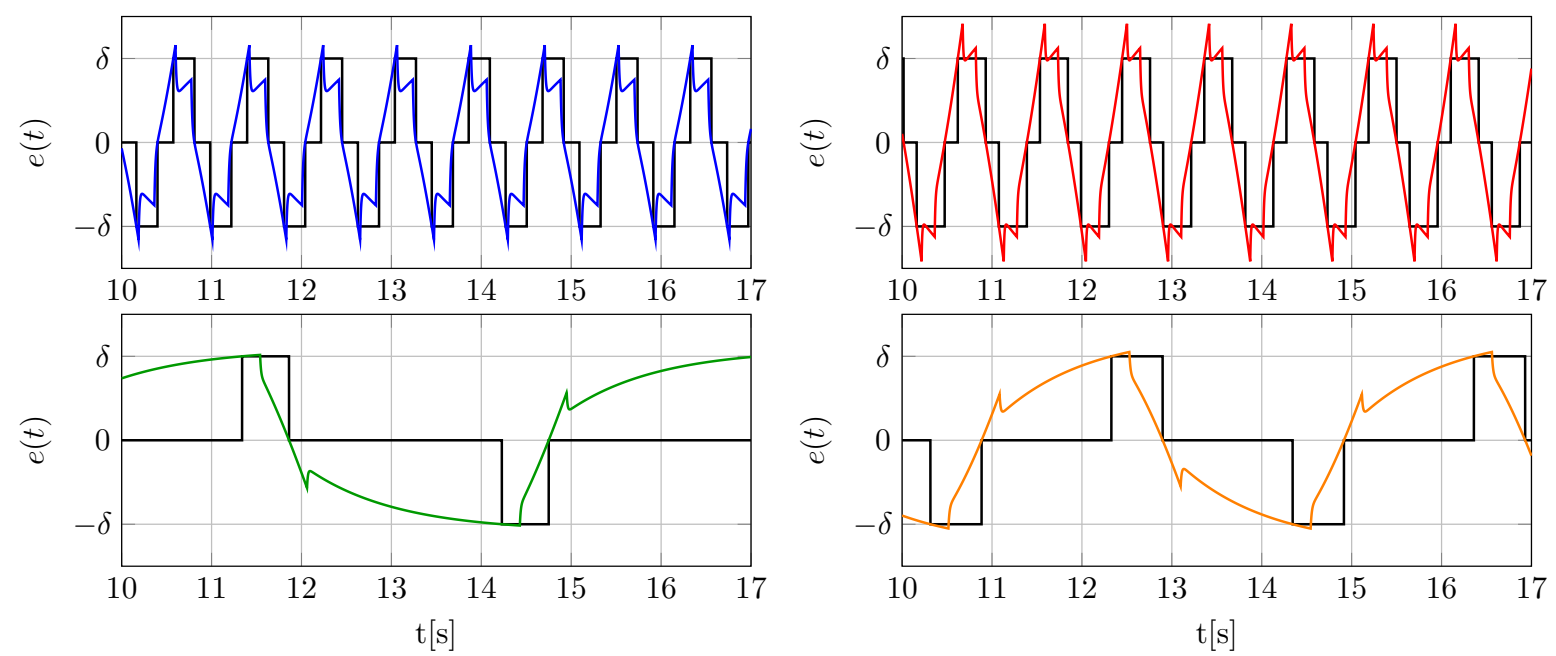

$\mathrm{t}[\mathrm{s}]$
— Ziegler-Nichols — Cohen-Coon — AMIGO — SRB

Figure 4.4: Steady-state oscillations of the system with $G(s)$ given by equation (4.2) with the PID parameters in Table 4.1.

Note that the previous expression depends on the real and imaginary part of the open-loop transfer function $\left(\Re\left\{G_{o l}\left(n \omega_{o}\right)\right\}, \Im\left\{G_{o l}\left(n \omega_{o}\right)\right\}\right)$ and on the number of levels $(m)$ crossed. It can be easily seen that as $m$ increases, the complexity of the calculations raise significantly because more oscillation conditions appear in equations (4.3) and (4.4), and the complexity of (4.5) increases too. For the purpose of this chapter the focus will be set on single leveled oscillations $(m=1)$, because it has been observed that by avoiding oscillation for $m=1$ no oscillations for $m>1$ take place. This result will be proved in section 4.6 , where multi-level oscillations are addressed. Thus, for practical reasons, this study will be focused on oscillations with $m=1$, whose existence conditions, obtained from equations (4.3) and (4.4) are

$$
E C_{p \mid m=1} \mid e\left(\rho_{p} \frac{T_{o}}{2}\right)= \begin{cases}\delta & \text { for } p=1 \\ 0 & \text { for } p=2\end{cases}
$$

and

$$
I C_{p \mid m=1} \mid \frac{d e\left(\rho_{p} \frac{T_{o}}{2}\right)}{d t}=\left\{\begin{array}{ll}
>0 & \text { for } p=1 \\
<0 & \text { for } p=2
\end{array} .\right.
$$

It is worth noticing that conditions (4.6) and (4.7) are sufficient and necessary for the existence of limit cycles with $m=1$. Consequently, if any of the previous equations is not fulfilled, then, steady-state oscillations do not appear in the system. Therefore, for the sake of simplicity and taking into account that the objective is to avoid limit cycles, the conditions in (4.6) will be considered to define a new robustness index to prevent oscillations. Evaluating (4.5) in $p=1,2$ and substituting the result in (4.6) taking into account that by definition $\rho_{2 m}=1$ 


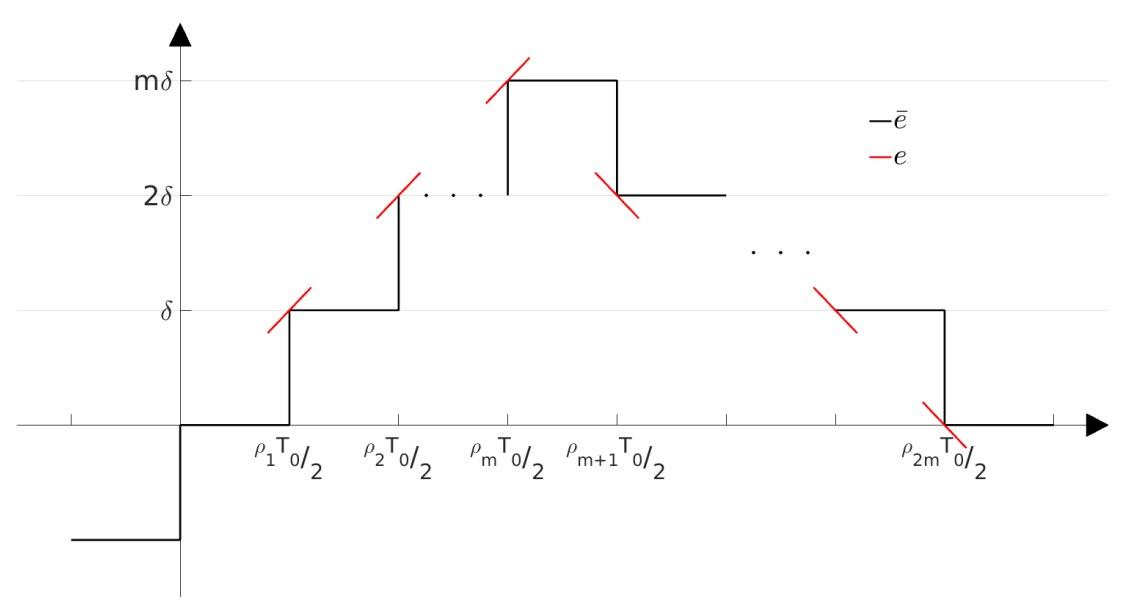

Figure 4.5: General form of an oscillation, which define the oscillation conditions in Tsypkin's method.

and renaming $\rho_{1}$ as $\rho$, the following equations are obtained:

$$
\begin{aligned}
& \sum_{n=1,3,5, \ldots}^{\infty} \frac{1}{n} \Re\left\{G_{o l}\left(j n \omega_{o}\right)\right\} \sin (n \pi \rho)+\frac{\pi}{4}=0 \\
& \sum_{n=1,3,5, \ldots}^{\infty} \frac{1}{n} \Im\left\{G_{o l}\left(j n \omega_{o}\right)\right\} \cos ^{2}\left(\frac{n \pi \rho}{2}\right)+\frac{\pi}{8}=0
\end{aligned}
$$

that finally can be rewritten as follows:

$$
\begin{gathered}
\Re\left\{G_{o l}\left(j \omega_{o}\right)\right\}=-\frac{\frac{\pi}{4}+\sum_{n=3,5 . .}^{\infty} \frac{1}{n} \sin (n \pi \rho) \Re\left\{G_{o l}\left(j n \omega_{o}\right)\right\}}{\sin (\pi \rho)}, \\
\Im\left\{G_{o l}\left(j \omega_{o}\right)\right\}=-\frac{\frac{\pi}{8}+\sum_{n=3,5 . .}^{\infty} \frac{1}{n} \cos ^{2}\left(\frac{n \pi \rho}{2}\right) \Im\left\{G_{o l}\left(j n \omega_{o}\right)\right\}}{\cos ^{2}\left(\frac{\pi \rho}{2}\right)} .
\end{gathered}
$$

For a given frequency $\omega_{o}$ and $\left.\rho \in\right] 0,1[$, the right hand members of the previous equations define a trajectory in the Nyquist plane which will be referred to as Tsypkin branch for $\omega_{o}$ $\left(B_{T}\left(\omega_{o}\right)\right)$.

$\left.B_{T}\left(\omega_{o}\right)=\left(-\frac{\frac{\pi}{4}+\sum_{n=3,5 .}^{\infty} \frac{1}{n} \sin (n \pi \rho) \Re\left\{G_{o l}\left(j n \omega_{o}\right)\right\}}{\sin (\pi \rho)},-\frac{\frac{\pi}{8}+\sum_{n=3,5 . .}^{\infty} \frac{1}{n} \cos ^{2}\left(\frac{n \pi \rho}{2}\right) \Im\left\{G_{o l}\left(j n \omega_{o}\right)\right\}}{\cos ^{2}\left(\frac{\pi \rho}{2}\right)}\right), \quad \forall \rho \in\right] 0,1[$

Then, a limit cycle oscillation with frequency $\omega_{o}$ will take place if the Tsypkin branch for 
$\omega_{o}$ intercepts the point $\left(\Re\left\{G_{o l}\left(j \omega_{o}\right)\right\}, \Im\left\{G_{o l}\left(j \omega_{o}\right)\right\}\right)$ of the Nyquist diagram, that is:

$$
\left(\Re\left\{G_{o l}\left(j \omega_{o}\right)\right\}, \Im\left\{G_{o l}\left(j \omega_{o}\right)\right\}\right)=B_{T}\left(\omega_{o}\right) .
$$

Using these definitions a robustness measure against limit cycles can be established as the minimum euclidean distance between the Nyquist points $\left(\Re\left\{G_{o l}(\omega)\right\}, \Im\left\{G_{o l}(\omega)\right\}\right)$ and their respective Tsypkin branches obtained for the same frequency $\omega$. This robustness measure will be referred to as Tsypkin margin $\left(M_{T}\right)$, and can be expressed as:

$M_{T}\left(\omega_{\text {min }}, \rho_{\text {min }}\right)=\sqrt{\left(\frac{\frac{\pi}{4}+\sum_{n_{\text {odd }}}^{\infty} \frac{1}{n} \sin \left(n \pi \rho_{\text {min }}\right) \Re\left\{G_{\text {ol }}\left(j n \omega_{\text {min }}\right)\right\}}{\sin \left(\pi \rho_{\text {min }}\right)}\right)^{2}+\left(\frac{\frac{\pi}{8}+\sum_{n_{\text {odd }}}^{\infty} \frac{1}{n} \cos ^{2}\left(\frac{n \pi \rho_{\text {min }}}{2}\right) \Im\left\{G_{\text {ol }}\left(j n \omega_{\text {min }}\right)\right\}}{\cos ^{2}\left(\frac{\pi \rho_{\text {min }}}{2}\right)}\right)^{2}}$,

being $\omega_{\min }$ and $\rho_{\min }$ the values of $\omega$ and $\rho$ for which the minimum distance is obtained.

The following examples illustrate the use of $M_{T}$ to predict the existence of limit cycle and the graphical interpretation of this new robustness margin.

Example 11. Non-oscillatory system. Consider the following FOPTD transfer function:

$$
G(s)=\frac{e^{-s}}{s+1}
$$

A PI controller with $K_{p}=0.84$ and $T_{i}=1.17$ is tuned using the SRB method [66]. A set of Tsypkin branches represented in Figure 4.6 has been obtained by evaluating equations (4.9) and (4.10) for $\rho \in] 0,1\left[\right.$ and $\omega \in\left[\frac{\omega_{c g}}{200}, \omega_{c g}\right]$, where $\omega_{c g}$ is the crossover frequency. In order to make the visualization easier, different colors are used to represent each point $\left(\Re\left\{G_{o l}(j \omega)\right\}, \Im\left\{G_{o l}(j \omega)\right\}\right)$ and its respective $B_{T}(\omega)$. The minimal distance between $\left(\Re\left\{G_{o l}(j \omega)\right\}, \Im\left\{G_{o l}(j \omega)\right\}\right)$ and $B_{T}(\omega)$, which has been highlighted with a dashed line, is $M_{T}=0.23$, obtained for $\omega=1.0191 \mathrm{rad} / \mathrm{s}$. Note that, even though there are several branches (the red ones) very close to some points of $G_{o l}$, these branches correspond to frequencies whose points $\left(\Re\left\{G_{o l}(j \omega)\right\}, \Im\left\{G_{o l}(j \omega)\right\}\right)$ (the red ones), are further than 0.23 .

Example 12. Oscillatory system. Consider the system in Example 10 and the PID obtained with SRB tuning procedure. Applying the concept of Tsypkin margin concludes that the system will oscillate because a distance $M_{T}=0$ has been obtained, as shown in Figure 4.7. The intersection between $G_{o l}(j w)$ and $B_{T}(w)$ takes place for $\rho=0.72$ and $\omega_{o}=1.55 \mathrm{rad} / \mathrm{s}, B_{T}(1.55)$ has been represented with a dashed line. The simulation shown in Figure 4.4 confirms this prediction.

Example 13. $\mathbf{M}_{\mathbf{T}}$ for well known tuning rules. The robustness measure $M_{T}$ can be used to determine the robustness against the oscillations produced by the SSOD structure presented in Figure 4.1, regardless of the method used for tuning the controller $C(s)$. As an extension of the work presented in [43], $M_{T}$ has been evaluated for three well known tuning methods, namely Ziegler-Nichols (ZN), Cohen-Coon (CC) and AMIGO tuning rules for both PI and PID. These 


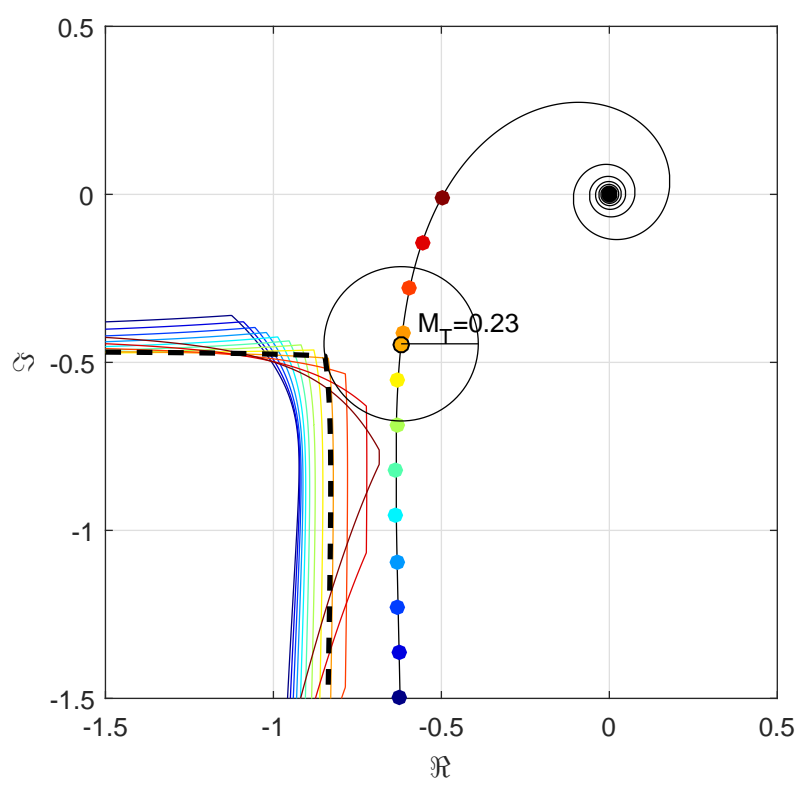

Figure 4.6: Nyquist diagram of $G_{o l}(\omega)$ and the Tsypkin band for a non-oscillating system $\left(M_{T}=\right.$ $0.23)$.

methods have been used to tune controllers for FOPTD models with different ratio delay $(L)$ and time constant $(\tau)$. The results are summarized in Figure 4.8.

It can be seen that the AMIGO tuning rules offer higher values of $M_{T}$ with lower ratios $L / \tau$ and then this measure tends to a value around 0.45, both for its PI and PID controller. The Ziegler-Nichols method offers lower values of $M_{T}$ than the AMIGO method for lower values of $L / \tau$, not being this the case for systems with higher ratio $L / \tau$ where the Ziegler-Nichols method offers the highest values of $M_{T}$. The Cohen-Coon method offers the lowest values of $M_{T}$ overall. It can also be observed that for all methods and controller types there are values of ratio $L / \tau$ with $M_{T}=0$. The range of values with $M_{T}=0$ is higher for PID controllers than for PI controller. Additionally, for each method the values of $M_{T}$ are higher for PI than for PID. This means that, in general, PI controllers have better robustness properties than PID when used with SSOD sampling strategy.

\subsection{SSOD-PID Controller Tuning}

In this section, a new tuning method for SSOD-PID controllers is proposed. It is worth noting that most of the studies about SSOD based control systems are focused on the PI case and there is a lack of results concerning the event based PID algorithm. From the discussion in Example 13, it is clear that the PI controllers have better robustness properties than PID to be used on the control scheme presented in Figure 4.1. However, the benefits of PID algorithm on improving the response of the control systems is no matter of doubt, and the case of SSOD-PID is not an exception, as it will be proved in this section. 


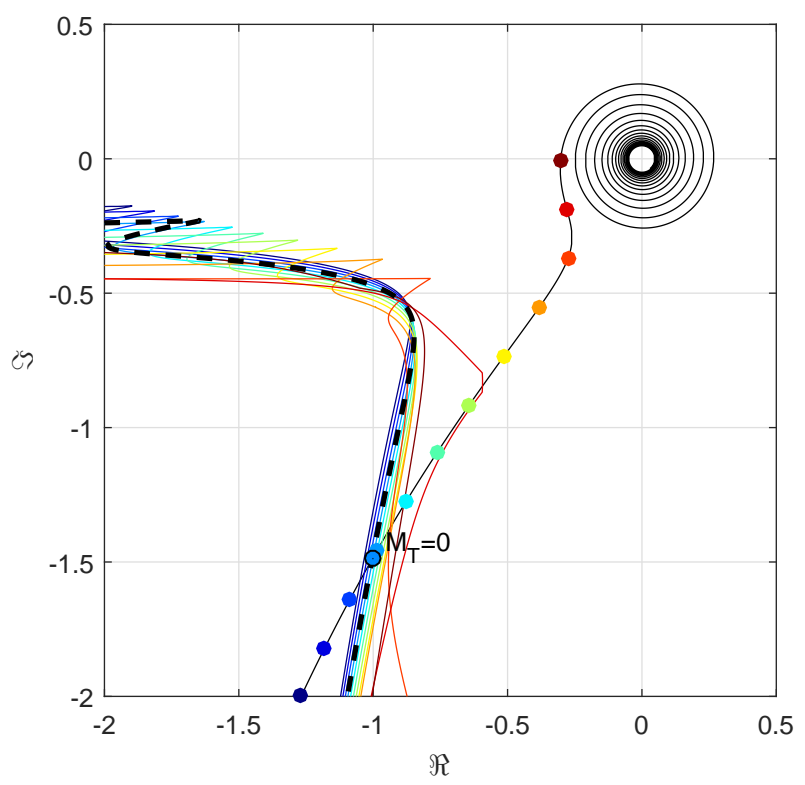

Figure 4.7: Nyquist diagram of $G_{o l}(\omega)$ and the Tsypkin band for a system with steady-state oscillations $\left(M_{T}=0\right)$.

The approach is focused on First Order Plus Time Delay (FOPTD) models, which allows approximating a wide range of actual industry processes, including those systems that do not admit the application of tuning methods based on the Describing Function, as the ones presented in $[60,56]$, due to its low filtering properties. When using FOPTD models a good technique for generalizing the results is expressing the transfer function in dimensionless form. The proposal is based on this feature, so before presenting the tuning method some preliminary results about the dimensionless approach are presented in the next subsection.

\subsubsection{Preliminary issues on dimensionless analysis}

Consider $G(s)$ in Figure 4.2 to be a FOPTD model. Without loss of generality, the network delay $t_{d}$ can be included in the time delay term $L$ :

$$
G(s)=\frac{K e^{-L s}}{\tau s+1} .
$$

A dimensionless representation of $G(s)$ is attained by making the transformation $\bar{s}=L s$ and $G(\bar{s}) / K$ :

$$
G(s)=\frac{K e^{-L s}}{\tau s+1} \stackrel{s=\frac{\bar{s}}{L}}{\Longrightarrow} G(\bar{s})=\frac{K e^{-\bar{s}}}{\frac{G(\bar{s}) / K}{L} \bar{s}+1} \stackrel{\bar{G}}{\Longrightarrow}(\bar{s})=\frac{e^{-\bar{s}}}{\frac{\tau}{L} \bar{s}+1} .
$$

The use of the dimensionless form of $G(s)$ makes the analysis easier because the results can be expressed in terms of the ratio $L / \tau$, the only parameter of $\bar{G}(\bar{s})$. That is the case for 


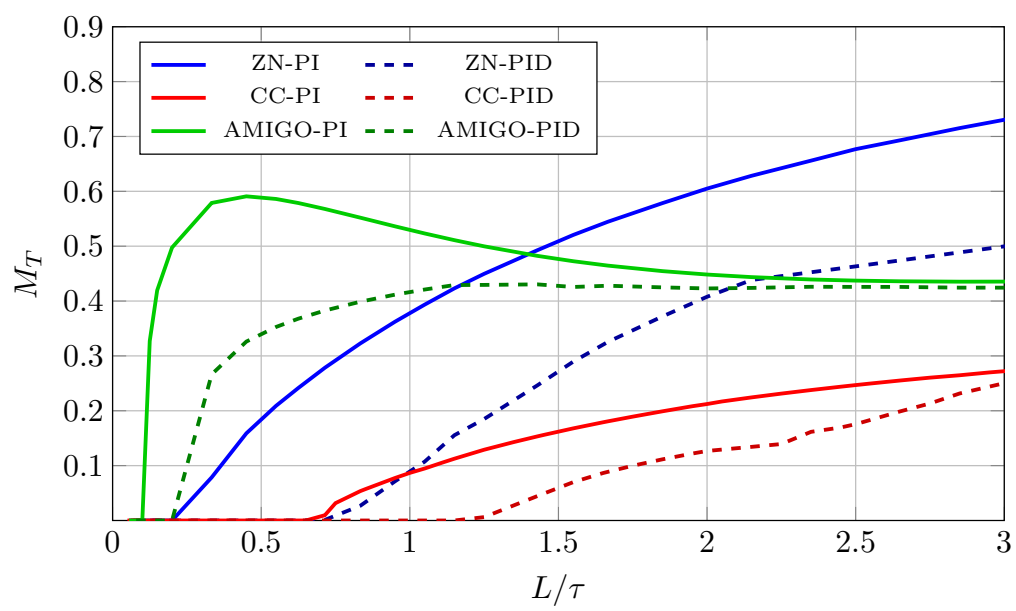

Figure 4.8: $M_{T}$ for FOPTD tuned with Ziegler-Nichols (ZN), Cohen-Coon (CC) and AMIGO tuning methods.

homogeneous tuning rules for PID controllers which can be expressed by the following equations:

$$
K_{p}=K^{-1} \phi_{1}\left(\frac{L}{\tau}\right)=K^{-1} \bar{K}_{p}, \quad T_{i}=L \phi_{2}\left(\frac{L}{\tau}\right)=L \bar{T}_{i}, \quad T_{d}=L \phi_{3}\left(\frac{L}{\tau}\right)=L \bar{T}_{d},
$$

Taking into account the previous equations, the dimensionless transfer function of the PID, obtained from equation (4.1), is:

$$
\begin{gathered}
C(s)=K_{p}\left(1+\frac{1}{T_{i} s}+\frac{T_{d} s}{\frac{T_{d} s}{N}+1}\right) \stackrel{s=\frac{\bar{s}}{L}}{\Longrightarrow} C(\bar{s})=K_{p}\left(1+\frac{1}{T_{i} / L \bar{s}}+\frac{T_{d} / L \bar{s}}{\frac{T_{d} / L \bar{s}}{N}+1}\right) \\
\bar{C}(\bar{s})=K C(\bar{s}) ; \quad \bar{C}(\bar{s})=\phi_{1}\left(1+\frac{1}{\phi_{2} \bar{s}}+\frac{\phi_{3} \bar{s}}{\frac{\phi_{3} \bar{s}}{N}+1}\right),
\end{gathered}
$$

where, for the sake of simplicity, the argument $L / \tau$ has been suppressed from $\phi_{1}, \phi_{2}$ and $\phi_{3}$. The dimensionless open-loop transfer function of the system in Figure 4.2 can be obtained using equations (4.12) and (4.14):

$$
\overline{G_{o l}}(\bar{s})=\bar{C}(\bar{s}) \bar{G}(\bar{s})=\phi_{1}\left(1+\frac{1}{\phi_{2} \bar{s}}+\frac{\phi_{3} \bar{s}}{\frac{\phi_{3} \bar{s}}{N}+1}\right) \frac{e^{-\bar{s}}}{\frac{\tau}{L} \bar{s}+1} .
$$

Furthermore, from equations (4.12) and (4.14):

$$
\overline{G_{o l}}(\bar{s})=\bar{C}(\bar{s}) \bar{G}(\bar{s})=(K C(\bar{s}))\left(\frac{G(\bar{s})}{K}\right)=C(\bar{s}) G(\bar{s})=G_{o l}(\bar{s})=G_{o l}(s L) .
$$


The use of homogeneous rules for tuning the PID controllers has important implications that can simplify the design of SSOD-PID for FOPTD systems. Concerning $M_{T}$, since its value only depends on the open-loop transfer function, and according to the equation (4.15) $\overline{G_{o l}}(\bar{s})=G_{o l}(s L)$, the value of Tsypkin margin calculated with $\overline{G_{o l}}(\bar{s})$, denoted as $\overline{M_{T}}$, holds the following relation with $M_{T}$ :

$$
\overline{M_{T}}\left(\overline{\omega_{\min }}, \rho_{\min }\right)=M_{T}\left(\omega_{\min } L, \rho_{\min }\right) .
$$

Thus, for FOPTD models tuned with homogeneous tuning rules, evaluating $M_{T}$ with the dimensionless open-loop transfer function $\overline{G_{o l}}(\bar{s})$ offers the same value of $M_{T}$ than with the dimensional open-loop transfer function. Moreover, the frequency $\omega_{\min }$ can be directly obtained from the dimensionless value $\overline{\omega_{\min }}$ as $\overline{\omega_{\min }}=\omega_{\min } L$.

When using a PID controller in structures with a SSOD sampler, another important issue to be considered in the design is the change $\delta_{u}$ in the control action produced by the changes $\delta$ on the sampled error signal $\bar{e}$. The value of $\delta_{u}$ is directly related to the smoothness of the control action and its limitation is important to avoid sudden changes in the control action that could harm the actuator or cause a malfunctioning of the control system. It should be taken into account that $\bar{e}$ is a noise free stair-like signal with steps of magnitude $\delta$, which are amplified by the controller in the same magnitude as a high frequency noise, according the following equation:

$$
\delta_{u}=K_{p}(1+N) \delta=C(\infty) \delta,
$$

where $K_{p}$ is the controller gain and $N$ the derivative filter coefficient. Concerning the selection of $N$ it is important to note that although a common practice in industry is to fix it at high values, some researches have recently shown that using $N$ as a free parameter allows balancing the noise amplification and the closed-loop performance (see $[26,34]$ ). A similar effect of $N$ in the case of the event-based system in Figure 4.1 has been reported in [58], where the selection of this parameter allows setting a trade-off between the control action jumps $\delta_{u}$ and the $I A E$ of the disturbance response.

The fact of using homogeneous tuning rules allows obtaining the value of $C(\infty)$ for a given system from the dimensionless model by dividing the obtained value of $\bar{C}(\infty)$ by the system gain:

$$
C(\infty)=K_{p}(1+N)=\frac{\phi_{1}}{K}(1+N)=\frac{\bar{C}(\infty)}{K}
$$

Finally, following the dimensionless approach, a simple relation can be extracted between the $I A E$ index for dimensional and dimensionless models, which is given by the following equation (see Appendix D.1):

$$
I A E=|K| L \cdot \overline{I A E} .
$$

In summary, when using homogeneous rules to tune a PID controller for a FOPTD system $G(s)$, the value of the controller parameters, $C(\infty)$ and $I A E$ (or other indexes) can be calculated 
Table 4.2: Summary of characteristics for the studied systems.

\begin{tabular}{c|cccc|cccc} 
& \multicolumn{5}{|c|}{ PI } & \multicolumn{4}{c}{ PID $(\mathbf{N}=\mathbf{1 0})$} \\
\cline { 2 - 9 } & $\mathbf{M}_{\mathbf{T}}$ & $\mathbf{I A E}_{\mathbf{p}}$ & $\mathbf{I A E}_{\mathbf{r}}$ & $\mathbf{C}(\infty)$ & $\mathbf{M}_{\mathbf{T}}$ & $\mathbf{I A E}_{\mathbf{p}}$ & $\mathbf{I A E}_{\mathbf{r}}$ & $\mathbf{C}(\infty)$ \\
\hline$\overline{\mathbf{G}}(\overline{\mathbf{s}})$ & 0.38 & 3.3168 & 3.3458 & 0.9 & 0.0927 & 1.6626 & 1.9092 & 13.2 \\
$\mathbf{G}_{\mathbf{1 , 2}}(\mathbf{s})$ & 0.38 & 6.6335 & 6.6916 & 0.9 & 0.0981 & 3.253 & 3.8184 & 13.2 \\
$\mathbf{G}_{\mathbf{2}, \mathbf{1}}(\mathbf{s})$ & 0.38 & 6.6335 & 3.3458 & 0.45 & 0.0927 & 3.253 & 1.9092 & 6.6 \\
$\mathbf{G}_{\mathbf{2}, \mathbf{2}}(\mathbf{s})$ & 0.38 & 13.2671 & 6.6916 & 0.45 & 0.0981 & 6.6505 & 3.8184 & 6.6
\end{tabular}

by scaling the results obtained with the dimensionless model $\bar{G}(\bar{s})$ using the equations (4.13), (4.18) and (4.19) respectively. Furthermore, the value of $M_{T}$ is the same as the obtained with $\bar{G}(\bar{s})$. To illustrate this idea the Example 14 is introduced.

Example 14. Consider three different FOPTD systems whose transfer functions are:

$$
G_{1,2}(s)=\frac{e^{-2 s}}{2 s+1}, \quad G_{2,1}(s)=\frac{2 e^{-s}}{s+1}, \quad G_{2,2}(s)=\frac{2 e^{-2 s}}{2 s+1} .
$$

The dimensionless model for these systems is $\bar{G}(\bar{s})=\frac{e^{-\bar{s}}}{\bar{s}+1}$. PI and PID controllers are tuned using the Ziegler-Nichols method, which is a well known homogeneous tuning rule. The resulting $M_{T}$, the disturbance IAE (IAE $)$, the reference IAE (IAE $)$ and $C(\infty)$ of each system are computed. The results are gathered in Table 4.2. This table shows how all the systems that have the same ratio $L / \tau$, have the same value of $M_{T}$, which confirms that this margin only depends on the ratio $L / \tau$. The relation of the other performance indexes $\left(I A E_{p}, I A E_{r}\right.$ and $\left.C(\infty)\right)$ with their dimensionless counterparts fulfill the described relations as expected.

\subsubsection{Tuning procedure}

According to the previous section, it is possible to calculate the controller parameters for any FOPTD transfer function $G(s)$ with a given quotient $L / \tau$ and evaluate its performance and robustness from the results obtained for the dimensionless model $\bar{G}(\bar{s})$ with the same value of $L / \tau$. In this section, the controller parameters and performance values are provided for a wide range of models $\bar{G}(\bar{s})$ taking into account an optimum tradeoff between the $\overline{I A E}$ performance index, the smoothness of the control action in terms of $\bar{C}(\infty)$ and the robustness measure to limit cycle $M_{T}$. From these values, it is possible to calculate the results for any system $G(s)$ with the same ratio $L / \tau$ using the equations (4.13), (4.18) and (4.19).

The approach is based on the tuning algorithm proposed in [66], which will be referred to as SRB method, which minimizes the disturbance $I A E$ index with restrictions on the phase and gain margins. The selection of this algorithm is based on three facts: 1) This method offers different robustness configurations expressed in terms of phase and gain margins. The definition of $M_{T}$ intuitively suggests a direct relation with the phase margin. Therefore, different values of $M_{T}$ can be obtained by changing the phase margin. 2) The derivative filtering coefficient $N$ is considered as a free design parameter, offering controllers with a smoother control action 
than those with high values of $N$. 3) According to [7], the minimization of the disturbance IAE, which depends on the plant sensitivity, assures the homogeneity of the tuning results obtained with this method.

It is worth noticing that SRB method has been originally developed for continuous-time controllers, but the objective is to extend it to systems with a SSOD sampling strategy, the one presented in Figure 4.1, by fulfilling the requirements on $M_{T}$ and $C(\infty)$. More precisely, the design must minimize the disturbance $I A E$ while meeting requirements on robustness to limit cycle $\left(M_{T}>M_{T_{r}}\right)$ and smoothness of the control action $\left(C(\infty)<C_{r}(\infty)\right)$ that formally can be written as in equation (4.20). Nevertheless, the relation between these requirements and the design parameters used in the SRB method, which involves phase and gain margins $\left(\phi_{m}, \gamma_{m}\right)$ and the derivative filtering coefficient $(N)$, is not evident.

$$
\begin{array}{ll}
\underset{K_{p}, T_{i}, T_{d}, N}{\operatorname{minimize}} & I A E=\int_{0}^{\infty}|e(t)| d t \\
\text { subject to } & M_{T} \geq M_{T_{r}}, \\
& C(\infty) \leq C_{r}(\infty)
\end{array}
$$

The proposed solution is to calculate the controllers using various combinations of phase and gain margins and derivative filtering coefficient. This produces a set of controllers with $M_{T}, \overline{I A E}, \bar{C}(\infty)$ and parameters $\overline{K_{p}}, \overline{T_{i}}, \overline{T_{d}}$. Then, the controller that minimizes $I A E$ fulfilling $M_{T}>M_{T_{r}}$ and $C(\infty)<C_{r}(\infty)$ is easily selected from this set. The validity of this approach lies on the fact that once the controllers are obtained for a batch of $\bar{G}(\bar{s})$, that is, varying the parameter $L / \tau$, the results can be used to obtain the controller for any FOPTD model with $L / \tau$ contained in the batch.

\subsubsection{Calculating the dimensionless controllers}

Following the previously described approach, dimensionless parameters were calculated using the SRB method for a set of systems $\bar{G}(\bar{s})$ with $L / \tau \in] 0,3]$ for all the possible combinations of $\phi_{m}=\left[50^{\circ}, 52.5^{\circ}, 55^{\circ}, 57.5^{\circ}, 60^{\circ}, 62.5^{\circ}, 65^{\circ}, 67.5^{\circ}, 70^{\circ}\right]$ and $N=[0,0.5,1,2,3,4,5,6,7,8$, $9,10,12,14,15,16,18,20]$. The gain margin was required to meet $\gamma_{m} \geq 6 \mathrm{~dB}$. The procedure is summarized in Figure 4.9. The values of $\phi_{m}$ and $N$ have been selected to guarantee a set of controllers dense enough in order to make the final controller as close as possible to the exact controller that minimizes the $I A E$ under the restrictions in $M_{T}$ and $C(\infty)$, i.e. the solution of the optimization problem given by equation (4.20). As said in the previous section, once the set of dimensionless parameters is calculated, it can be used to obtain the controller for any FOPTD model with $L / \tau \in] 0,3]$, in consequence, the proposed griding of $\phi_{m}$ and $N$ does not suppose a computation overload for the tuning of each controller. It is also important to remark that the phase and gain margins used as requirements are maintained from the dimensionless model to the dimensional one, the only difference between the dimensionless and the dimensional cases is the 


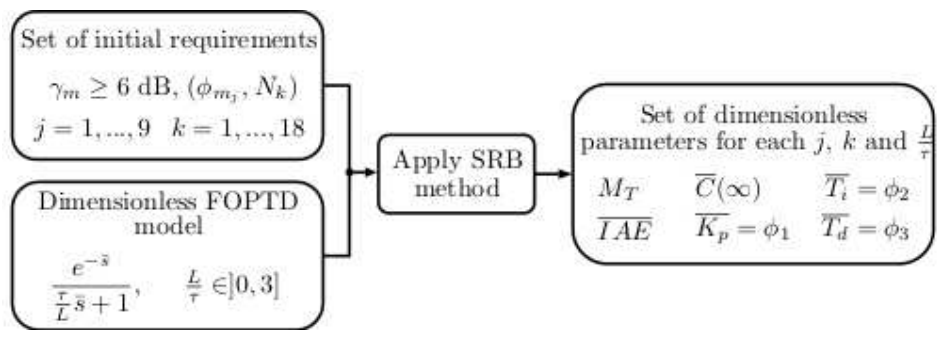

Figure 4.9: Summary of the calculus to obtain the dimensionless set of parameters for the controllers.

frequency at which these margins are measured due to the variable change $\left(j \overline{\omega_{\min }}=j \omega_{\min } L\right)$. Therefore, in addition to the required characteristics $M_{T_{r}}$ and $C_{r}(\infty)$, all the controllers of the set have at least $\phi_{m}=50^{\circ}$ and $\gamma_{m}=6 \mathrm{~dB}$.

Figure 4.10 shows the evolution of the margin $M_{T}$ with the ratio $L / \tau$ for some representative values of $\phi_{m}$ and $N$. Several interesting conclusions rise from this figure. Firstly, it can be seen that for systems close to a first order model (low values of $L / \tau$ ), $M_{T}=0$. Then, for given values of $L / \tau$, depending on $\phi_{m}$ and $\gamma_{m}, M_{T}$ increases quickly, reaching its maximum and then decreasing asymptotically to a final value. Secondly, the figures show that the higher the phase margin, the higher $M_{T}$. This confirms the intuition about the relation between these parameters, based on the fact that both measures are related with the robustness of the system. Finally, the relation between the filtering coefficient $N$ and the robustness to limit cycle is clearly shown: increasing the value of $N$ generally reduces $M_{T}$. In addition, it can be seen that it does not exist much difference between the values of $M_{T}$ obtained for $N=10,15$ and 20, thus, regarding $M_{T}$, increasing $N$ in a range further than 10 does not change significantly the robustness of the system. Summarizing, in the majority of the considered cases, incrementing $\phi_{m}$ tends to increase $M_{T}$, but increasing $N$ tends to decrease $M_{T}$.

Figures 4.11 and 4.12 show the values of $\overline{I A E}$ and $\bar{C}(\infty)$ for different values of $L / \tau$. Only the cases for which $M_{T} \neq 0$ have been represented. The worst results of $\overline{I A E}$ are obtained for the PI controllers $(N=0)$ and this index improves as $N$ rises, however, as in the case of $M_{T}$, no significant improvement is observed regarding the $I A E$ for those controllers with values of $N>10$. On the other hand, the effect of $\phi_{m}$ on the $\overline{I A E}$ is not significant for the PID controllers for any value of $N$. The same can not be said for the PI cases, whose values of $\overline{I A E}$ for low ratios $L / \tau$ clearly get worse with the increment of $\phi_{m}$. Regarding $\bar{C}(\infty)$, it can be seen how, as expected, increasing $N$ increases $\bar{C}(\infty)$, which increases significantly the value of $\delta_{u}$. Furthermore, the variations of $\phi_{m}$ barely affect this parameter.

\subsubsection{Software tool for tuning}

As a result of the calculation described on the previous section, 162 controllers $\left(\overline{K_{p}}, \overline{T_{i}}\right.$ and $\overline{T_{d}}$ ) have been obtained for each value of $\left.\left.L / \tau \in\right] 0,3\right]$, by taking all the possible combinations of $\phi_{m}=\left[50^{\circ}, 52.5^{\circ}, 55^{\circ}, 57.5^{\circ}, 60^{\circ}, 62.5^{\circ}, 65^{\circ}, 67.5^{\circ}, 70^{\circ}\right]$ and $N=[0,0.5,1,2,3,4,5,6,7$, 

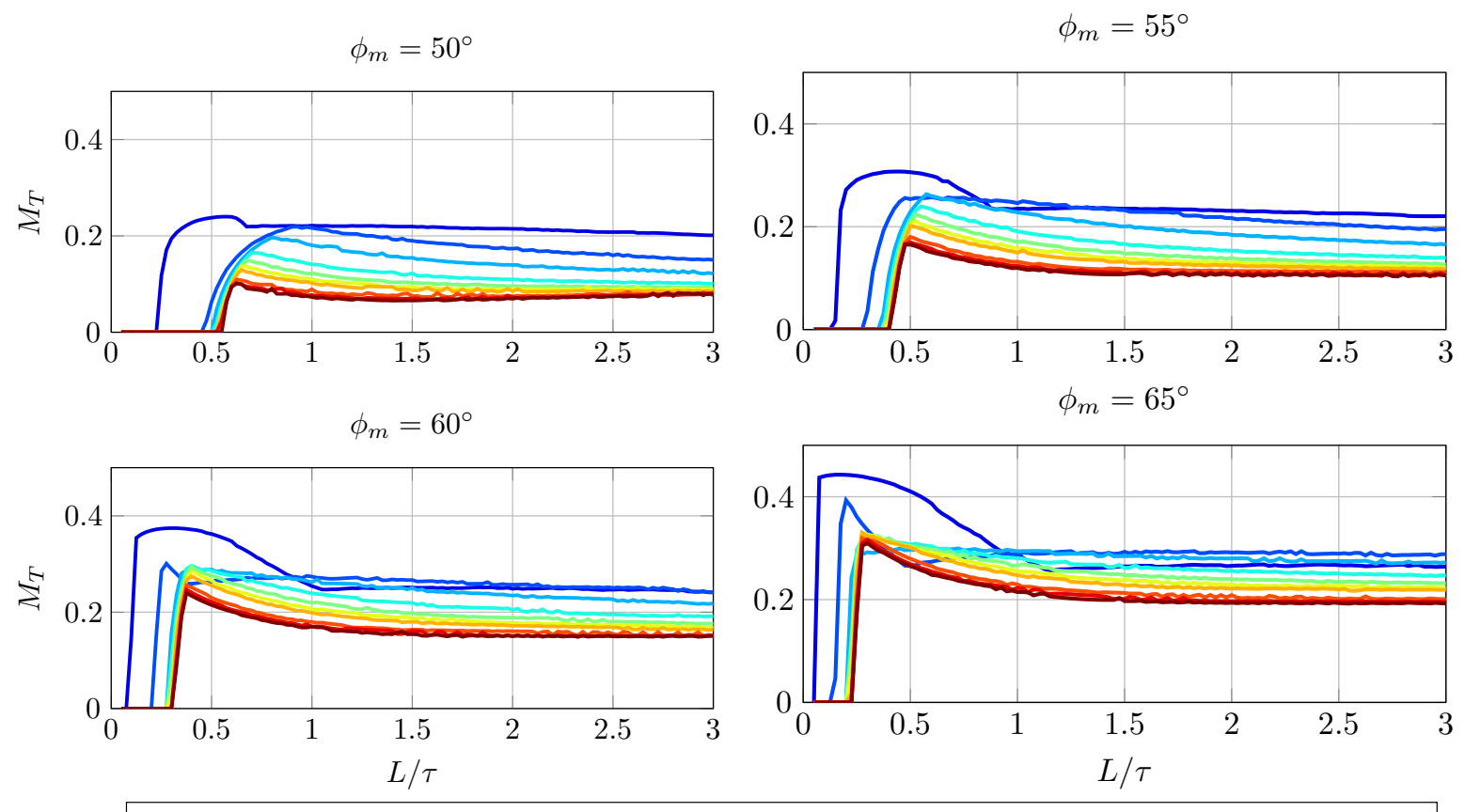

$\longrightarrow \mathrm{N}=0-\mathrm{N}=0.5-\mathrm{N}=1-\mathrm{N}=2-\mathrm{N}=3 \longrightarrow \mathrm{N}=4-\mathrm{N}=5-\mathrm{N}=10 \longrightarrow \mathrm{N}=15-\mathrm{N}=20$

Figure 4.10: Evolution of $M_{T}$ with $L / \tau$ for different configurations of $\phi_{m}$ and $N$.
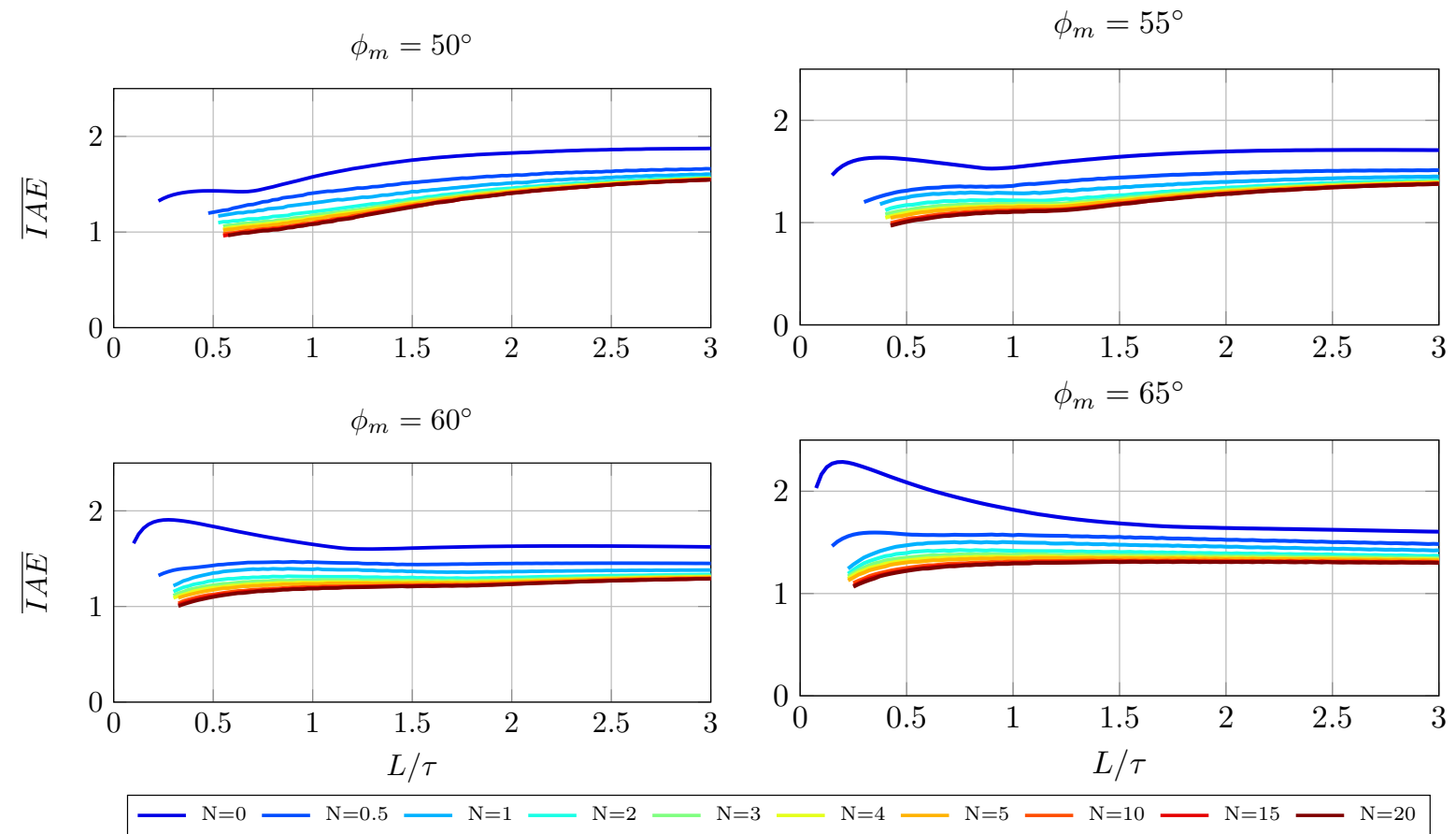

Figure 4.11: Obtained values of $\overline{I A E}$ for the considered batch for different configurations of $\phi_{m}$ and $N$. 

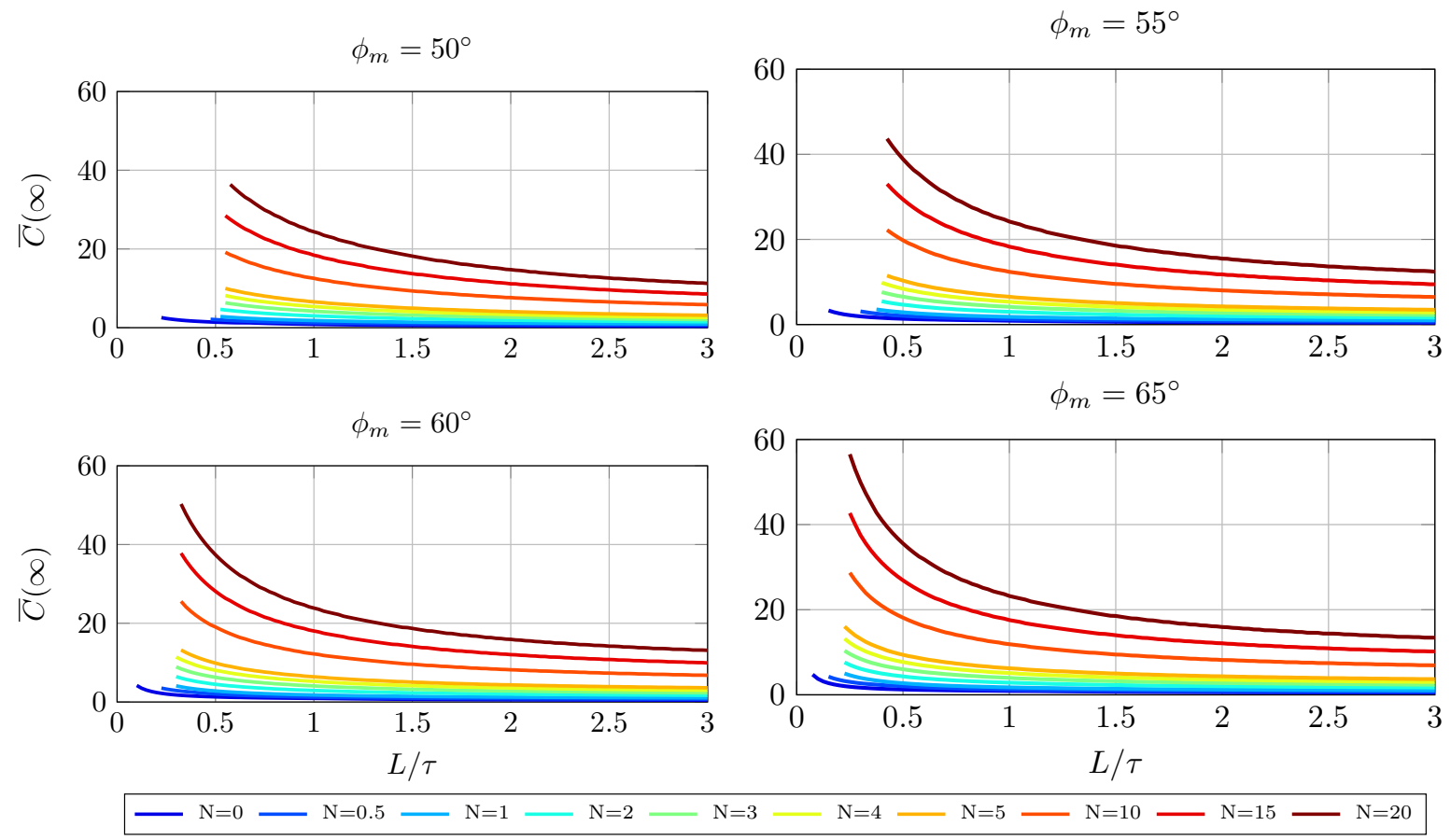

Figure 4.12: Obtained values of $\bar{C}(\infty)$ for the considered batch for different configurations of $\phi_{m}$ and $N$.

$8,9,10,12,14,15,16,18,20]$. Their respective values of $M_{T}, \overline{I A E}$ and $\bar{C}(\infty)$ have also been obtained. In order to manage this information more efficiently, a software tool was developed in the context of article [44] to facilitate the selection of the controller that minimizes the $I A E$ while fulfilling the constraints $M_{T}>M_{T_{r}}$ and $C(\infty)<C_{r}(\infty)$. It should be noted in Figures 4.10, 4.11 and 4.12 that smooth relations exist between the design parameters $\left(\phi_{m}\right.$ and $N)$ and the robustness/performance indexes $\left(M_{T}, \overline{I A E}\right.$ and $\left.\bar{C}(\infty)\right)$. This indicates that the proposed tuning procedure is not too sensitive to these parameters and therefore the solution obtained from the pre-calculated set of dimensionless controllers will be close to the actual optimal solution.

The tuning procedure using the set of dimensionless parameters pre-calculated in section 4.4.3 has been implemented in a Java application. The procedure is summarized in Figure 4.13. For a given FOPTD model and requirements $M_{T_{r}}$ and $C_{r}(\infty)$ the quotient $L / \tau$ is calculated and the controllers for this value of $L / \tau$ fulfilling the constrains $M_{T}>M_{T_{r}}$ and $\bar{C}(\infty) / K<C_{r}(\infty)$ are filtered. Then the controller with minimum $\overline{I A E}$ is selected and the dimensional parameters $K_{p}, T_{i}$ and $T_{d}$ are calculated using equations (4.13). If an arbitrary model is defined, the tool first obtains a FOPTD approximation, and calculates the controller using that approximation. The robustness margins and the response simulation, however, correspond to the original system, therefore it is easy to check the effect of approximate modeling on the design. The software tool can be freely downloaded from:

https://sites.google.com/a/uji.es/freepidtools/ssodTsypkinPid. 


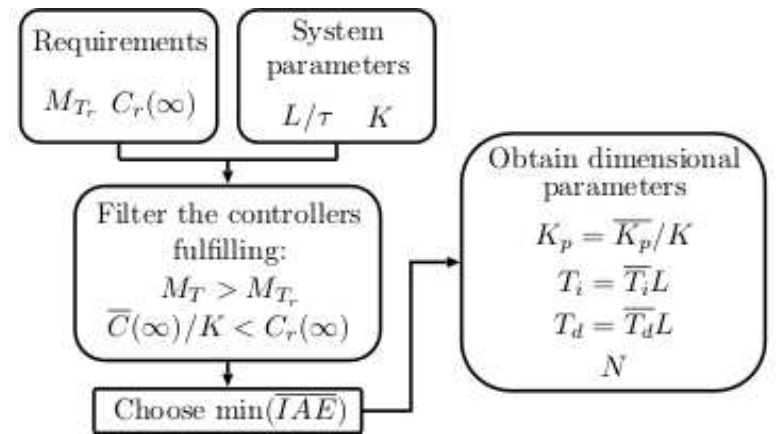

Figure 4.13: Summary of the controller design procedure.

The use of the application is introduced through the following example.

Example 15. Consider for this example a system whose transfer function is:

$$
G(s)=\frac{1}{\left(s+\frac{1}{3}\right)(s+1)(s+3)} .
$$

The application consists of a main window including several tabs with different functionality. Firstly, the process model must be defined in the Plant_definition tab, Figure 4.14. As commented before, since this model is not a FOPTD transfer function, a FOPTD approximation is calculated which will be used to obtain the controller parameters. The PID design is carried out in the PID_design tab, Figure 4.15, by fixing the desired value $M_{T_{r}}$ and $C(\infty)$. For this example it has been considered $M_{T_{r}} \geq 0.1$ and $C(\infty) \leq 20$. Then a button allows obtaining the PID controller parameters that minimize the IAE while fulfilling these constraints. The design results evaluated over the original systems, not the FOPTD approximation, are also shown in this tab. It can be seen how $M_{T}=0.32$ is greater than $M_{T_{r}}$ and that the value of $C(\infty)=18.28$ stays below the maximum required. The tool also shows the Tsypkin band that defines the margin $M_{T}$, and the complete set of Tsypkin bands if desired.

A separate window shows the response of the controlled system to a step change in the setpoint and in the disturbance input, see Figure 4.16, allowing to select the value of the SSOD parameter $\delta$, which has been fixed to 0.1 .

If the user wants to find another controller, the new restrictions must be introduced and the application will compute the result. For example, consider now that the requirement on the control action changes to $C_{r}(\infty) \leq 2$, then this parameter is introduced and the application computes the resultant controller as it is shown in Figure 4.1\%. Here, it can be seen that the new controller, which is a PI controller, fulfills the requirements on $M_{T}$ and $C(\infty)$, but the disturbance IAE has risen (from 1.37 to 1.86), as it can be appreciated in Figure 4.18.

\subsubsection{Tuning table}

Alternatively to the software tool presented in the previous section, the tuning can also be carried out using the table in Appendix C.1, which has been obtained for specific requirements 


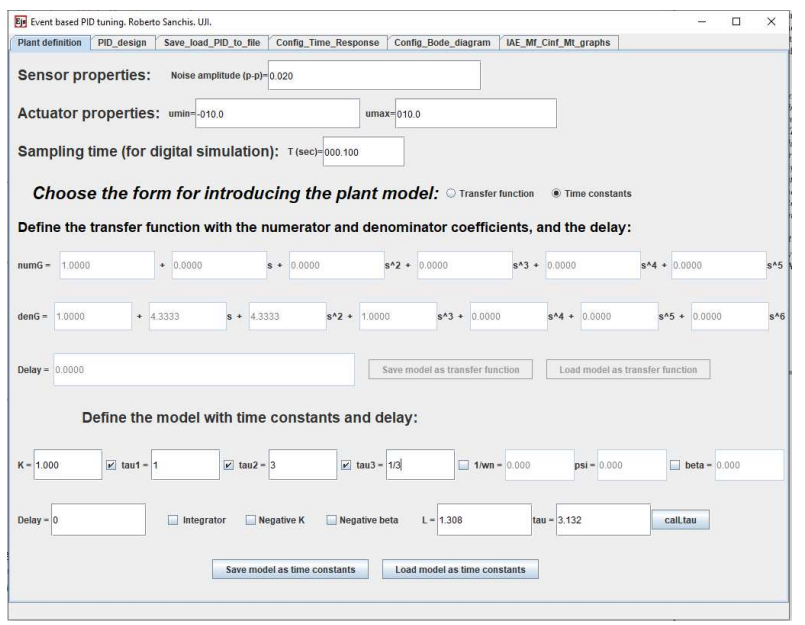

Figure 4.14: Software tool process definition window.

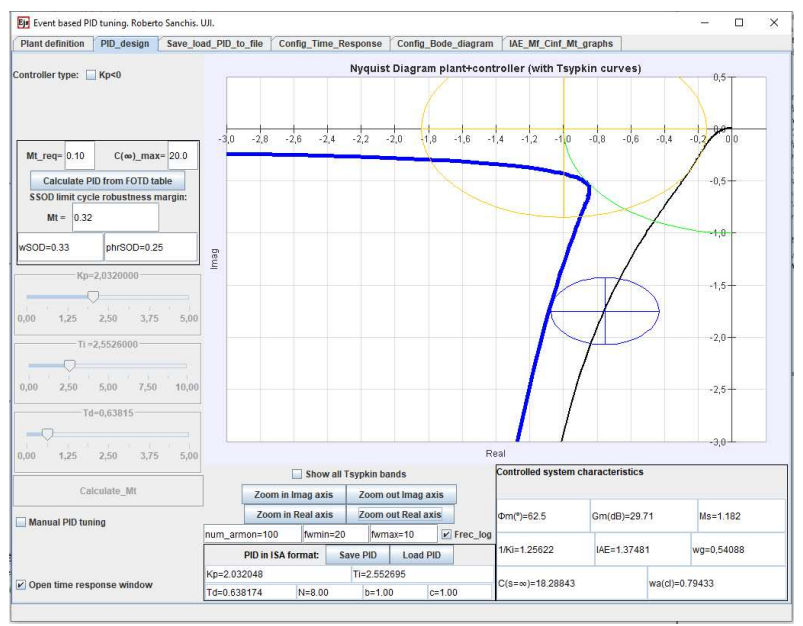

Figure 4.15: Software tool PID design window. 


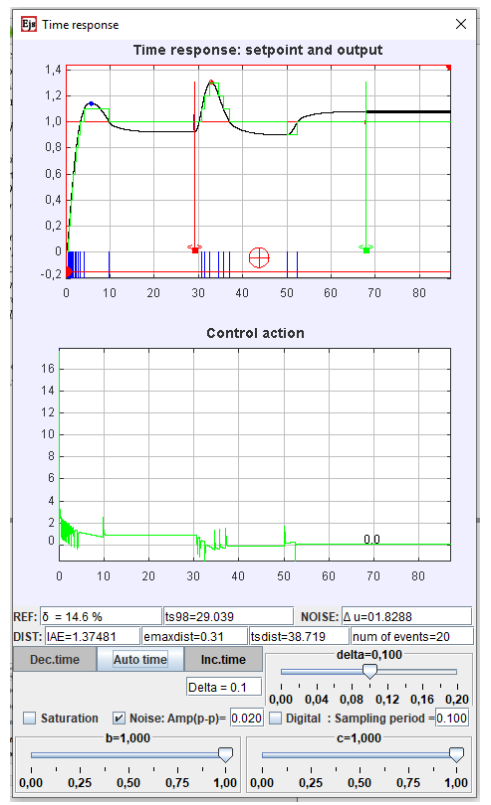

Figure 4.16: Software tool time response window.

on $\bar{C}(\infty)$ and $M_{T_{r}}>0.2$. Of course, this option is not as flexible and interactive as using the application, but it provides a very simple way for tuning the controller once the FOPTD model of the system is known.

The table provides the dimensionless parameters $\overline{K_{p}}, \overline{T_{i}}$ and $N$ for different values of the ratio $L / \tau$. The value of $\overline{T_{d}}$ has been omitted since its value is calculated as $\overline{T_{d}}=\overline{T_{i}} / 4$. The empty entries in the table mean that it is not possible to obtain a controller with the SRB method that fulfills the specified requirements. For the entries where a character $*$ appears, the controller parameters are the same as those obtained for the precedent value of $\bar{C}(\infty)$, that is, the parameters in the columns at the left of the position marked with $*$. The following example illustrates how the different proposed controllers in the table behave and some guidelines to choose them.

Example 16. Consider a FOPTD system whose transfer function is

$$
G(s)=\frac{0.5 e^{-2 s}}{5 s+1} .
$$

By using the proposed tuning table a robustness of $M_{T_{r}}>0.2$ is obtained, and each of the controllers has different levels of control action variations due to changes of $\delta$ in the sampled signal. A measurement gaussian noise is assumed, whose effect is mostly avoided by the choice of the SSOD thresholds $\delta=0.1$.

For transfer function (4.21) the ratio $L / \tau$ is 0.4 . Searching in Table C.1 the corresponding row, 4 controllers with different values of $\bar{C}(\infty)$ can be found. Each controller is denoted as $C_{i}$ where $i$ is the position of the controller when reading the table from left to right. The parameters 


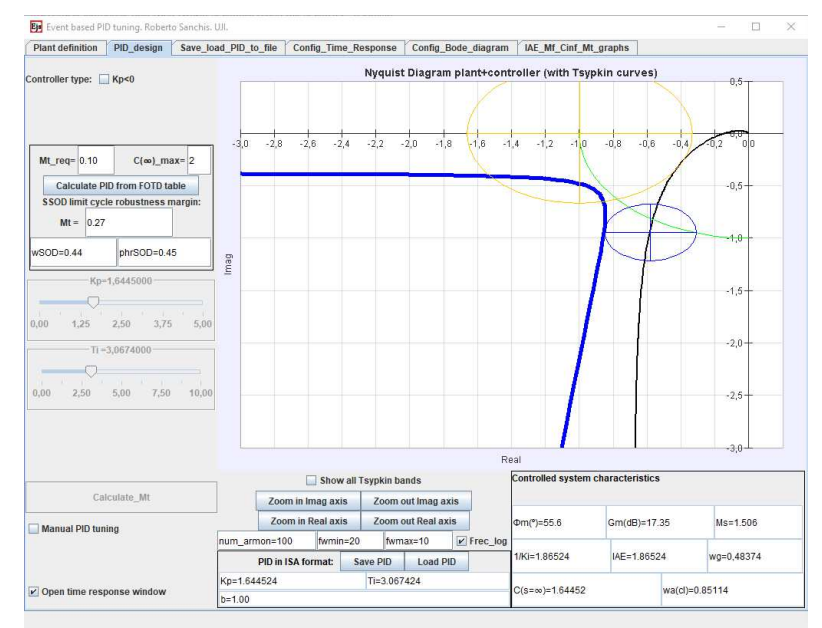

Figure 4.17: Software tool PID design window with modified parameters.

of the different controllers are expressed in a dimensionless way, thus, these values must be converted to dimensional parameters using the expressions in equation (4.13).

Figure 4.19 shows the open-loop transfer function for all the cases with their respective Tsypkin margin, proving that the robustness requirements have been fulfilled. Additionally, Figure 4.20 shows the closed-loop response to reference and disturbance step changes on $t=1 \mathrm{~s}$ and $t=25$ s respectively for all the cases. These systems do not present limit cycle oscillations, as it was expected. The controllers with higher values of $C(\infty)$, which have lower values of IAE, result in faster responses but paying the price of having more abrupt changes in the control action (as shown in Figure 4.21), as a result of higher derivative filter coefficients. This is more clear observing the response of the system controlled with $C_{1}$, which is a PI controller, with regard to the others, which are PID.

The example shows that it is not reasonable to choose an excessively high value of $\bar{C}(\infty)$, because the improvement in the disturbance IAE is small for a high increase in control action bump. For example, controllers $C_{3}$ and $C_{4}$ have a very similar temporal response (IAE) but the changes in control action produced by $C_{4}$ are significantly higher than those produced by the controller $C_{3}$. The value of $\delta_{u}$ for $C_{3}$ is $\delta_{u}=K_{p}(1+N) \delta=3.74(1+4) 0.1=1.87$, and $\delta_{u}=8.694$ for $C_{4}$, resulting in a more aggressive control action.

\subsection{Effect of the FOPTD approximation}

The tuning procedure presented in the previous sections is based on using a FOPTD model. However, the behavior of many actual industrial processes do not correspond strictly to this kind of models. In this sense, it is important to evaluate the effect of this approximation on the final results with the original system, especially on $M_{T}$ since its value determines the presence of limit cycle oscillations. To shed light about this issue, it has been considered the following 


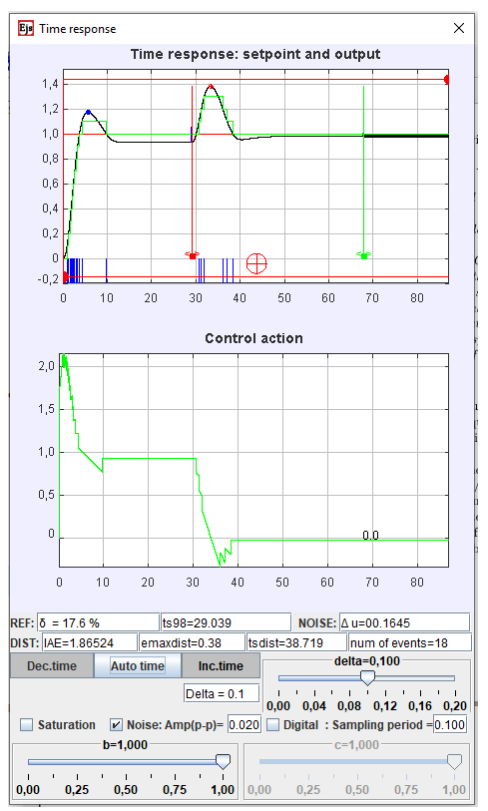

Figure 4.18: Software tool time response window with modified parameters.
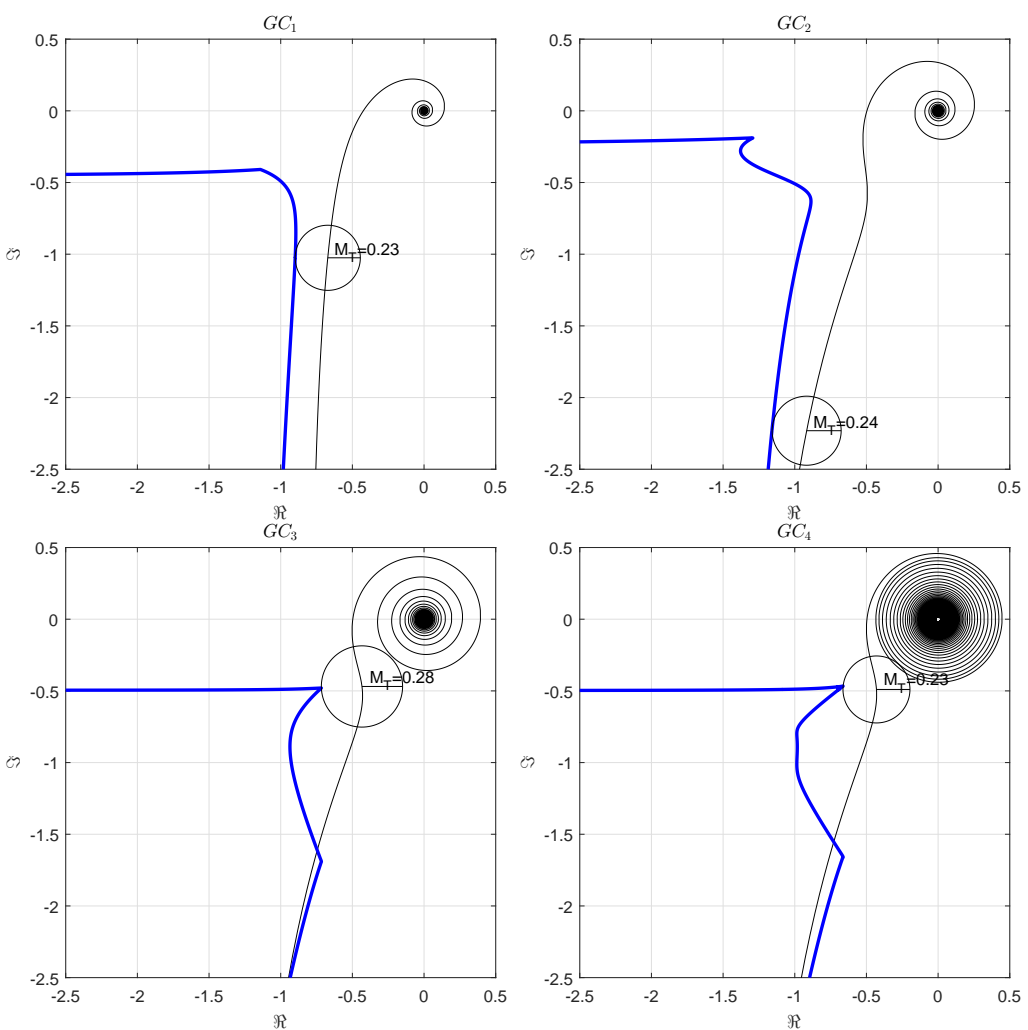

Figure 4.19: Nyquist plot of the open-loop transfer function with all the controllers and their minimum Tsypkin branch. 

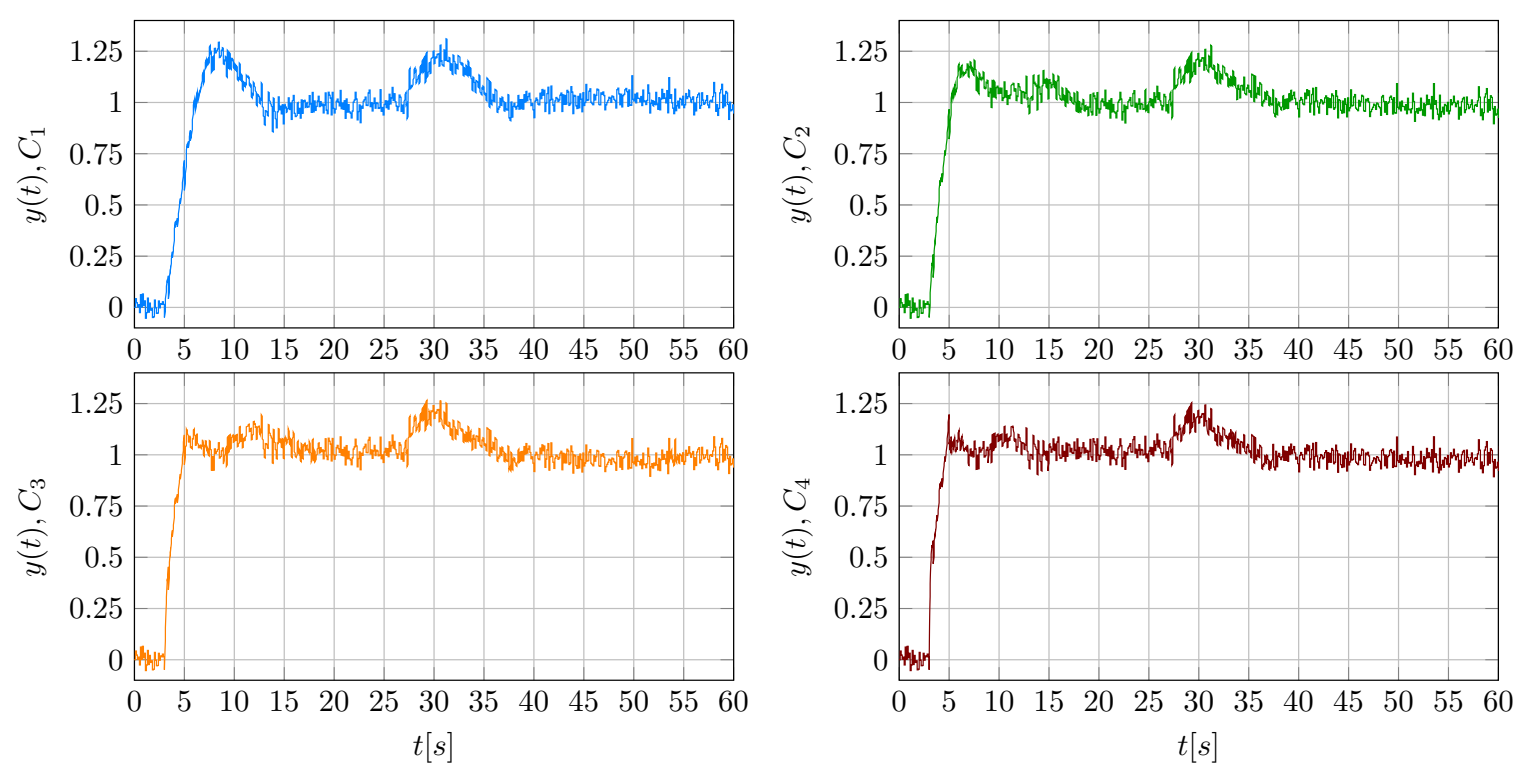

Figure 4.20: Disturbance response for the considered system with all the controllers.

batch of 95 models widely used to evaluate the performance of PID controllers, which represent most of the common dynamics in real applications, [5]:

$$
\begin{aligned}
& G(s)=\frac{e^{-s}}{(T s+1)^{2}}, \\
& T=0.3,0.5,0.7,1,1.3,1.5,2,4,6,8,10,20,50,100,200,500 \\
& G(s)=\frac{1}{(s+1)(T s+1)^{2}}, \\
& T=0.05,0.1,0.2,0.5,2,5,10 \\
& G(s)=\frac{1}{(s+1)^{n}}, \\
& n=3,4,5,6,7,8 \\
& G(s)=\frac{1}{(s+1)(\alpha s+1)\left(\alpha^{2} s+1\right)\left(\alpha^{3} s+1\right)}, \\
& \alpha=0.1,0.2,0.3,0.4,0.5,0.6,0.7,0.8,0.9 \\
& G(s)=\frac{T e^{-L_{1} s}}{\left(T_{1} s+1\right)(T s+1)}, \quad T_{1}+L_{1}=1, \\
& T=1,2,5,10, \quad L_{1}=0.01,0.02,0.05,0.1,0.3,0.5,0.7,0.9,1 \\
& G(s)=\frac{1-\alpha s}{(s+1)^{3}}, \\
& \alpha=0.1,0.2,0.3,0.4,0.5,0.6,0.7,0.8,0.9,1,1.1 \\
& G(s)=\frac{1}{(s+1)\left((s T)^{2}+1.4 s T+1\right)}, \\
& T=0.1,0.2,0.3,0.4,0.5,0.6,0.7,0.8,0.9,1
\end{aligned}
$$

Controllers with $M_{T} \geq 0.1$ and without constraint on $C(\infty)$ have been obtained for all these models using a FOPTD approximation. The values of $M_{T}$ calculated with both the FOPTD 

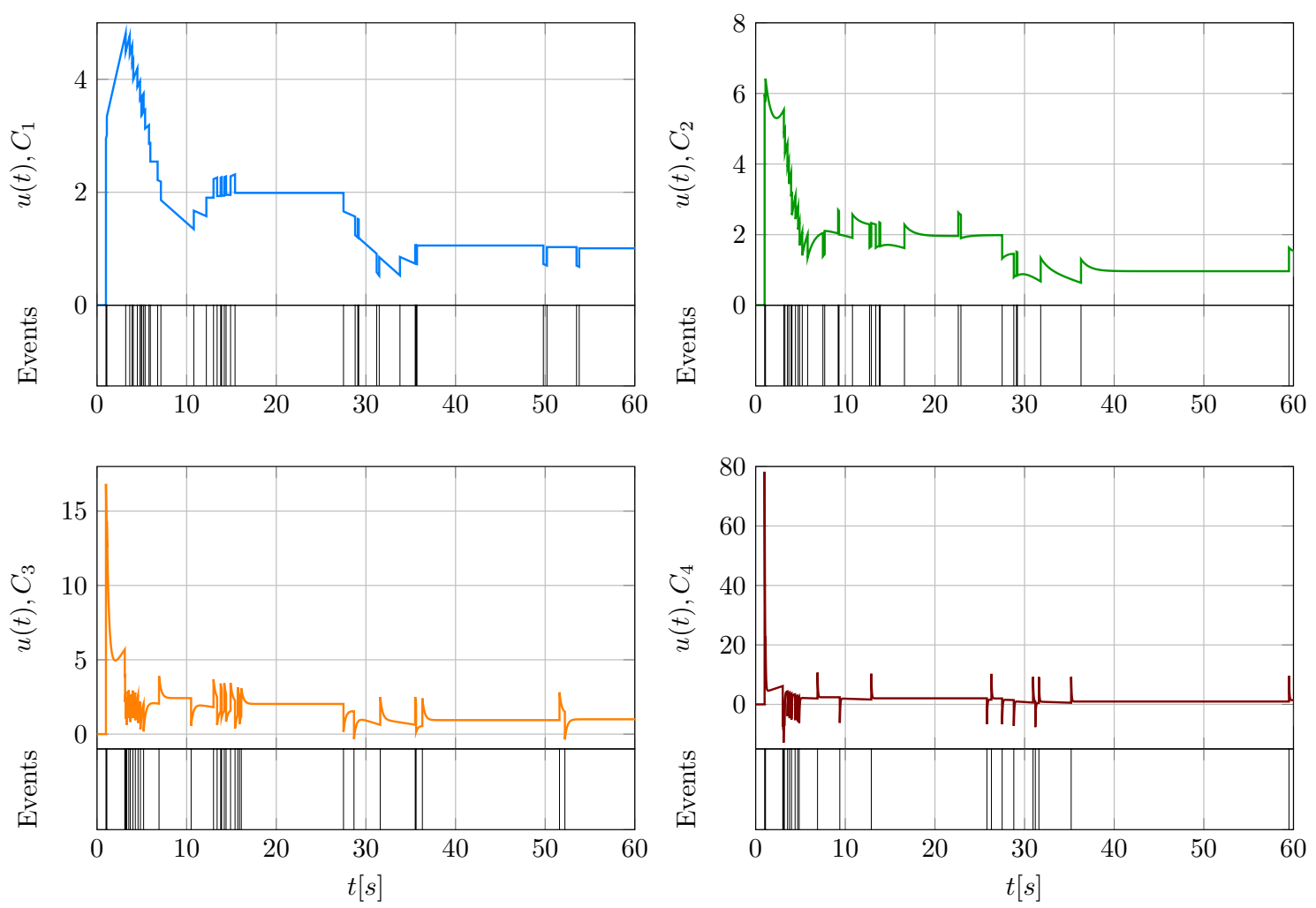

Figure 4.21: Control action and events generated by crossing the SSOD levels in black for the considered system with all controllers.

and the original transfer functions are presented in Figure 4.22. It can be seen that the final $M_{T}$ of the original system with the controller calculated for its FOPTD approximation is greater than the $M_{T}$ of the FOPTD approximation. Therefore, the robustness to limit cycle is assured when using controllers designed with the procedure presented in this chapter.

\subsection{Multi-level oscillations}

In the previous sections, single leveled oscillations with $m=1$ have been characterized using the robustness measure $M_{T}$ and a tuning method has been proposed to avoid this kind of limit cycles. Nevertheless, the question if by avoiding oscillations with $m=1$ is enough to ensure robustness to oscillations with $m>1$ has not been addressed yet. In this sense, some results were presented in [56] based on the shape of the describing function of the SSOD sampler, however, as commented earlier, the filtering hypothesis that this technique assumes as true is not fulfilled for FOPTD systems. Thus, the study of multi-level oscillation $(m>1)$ must follow the same ideas as that for $m=1$, which was presented in section 4.3.

As commented in section 4.3, the complexity of the calculation based on Tsypkin's conditions for oscillation raises significantly with $m$. As an example, consider the case $m=2$, whose 

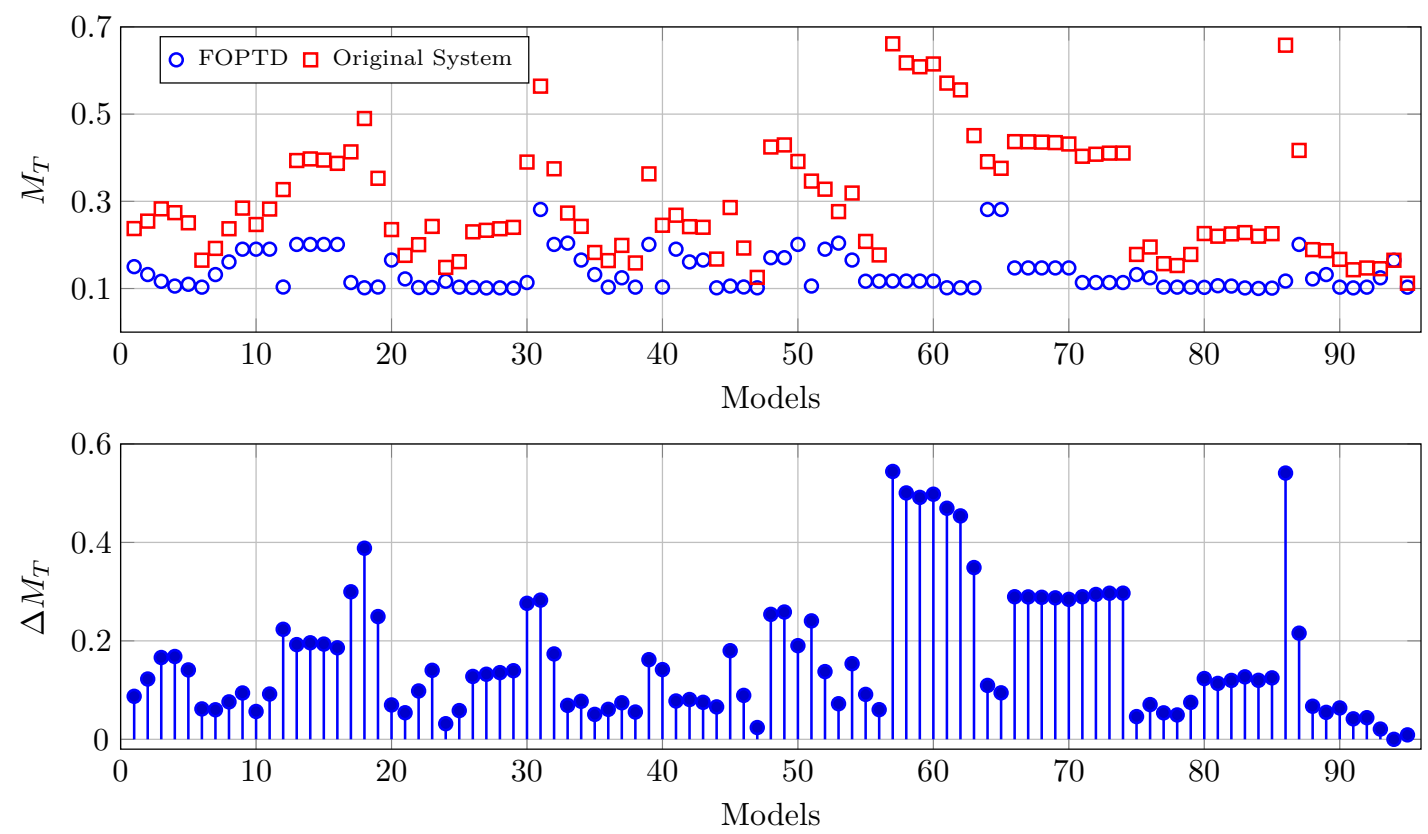

Figure 4.22: $M_{T}$ and $\Delta M_{T}$ for the considered systems.

oscillation conditions derived from equation (4.6) are:

$$
E C_{p \mid m=2} \mid e\left(\rho_{p} \frac{T_{0}}{2}\right)= \begin{cases}\delta & \text { for } p=1 \\ 2 \delta & \text { for } p=2 \\ \delta & \text { for } p=3 \\ 0 & \text { for } p=4\end{cases}
$$

Evaluating equation (4.5) for $m=2$ it can be obtained that:

$$
\begin{aligned}
& e\left(\rho_{p} \frac{T_{0}}{2}\right)=-\frac{4 \delta}{\pi} \sum_{n_{\text {odd }}}^{\infty} \frac{1}{n}\left(\Re\left\{G_{\text {ol }}\left(n \omega_{0}\right)\right\}\right.\left(\sin \left(\frac{n \pi}{2}\left(\rho_{2}-\rho_{1}\right)\right) \cos \left(n \pi\left(\frac{\rho_{2}+\rho_{1}}{2}-\rho_{p}\right)\right)\right. \\
&+2 \sin \left(\frac{n \pi}{2}\left(\rho_{3}-\rho_{2}\right)\right) \cos \left(n \pi\left(\frac{\rho_{3}+\rho_{2}}{2}-\rho_{p}\right)\right) \\
&+\left.\left.\sin \left(\frac{n \pi}{2}\left(\rho_{4}-\rho_{3}\right)\right) \cos \left(n \pi\left(\frac{\rho_{4}+\rho_{3}}{2}-\rho_{p}\right)\right)\right)\right) \\
&+\frac{4 \delta}{\pi} \sum_{n_{\text {odd }}}^{\infty} \frac{1}{n}\left(\Im \{ G _ { \text { ol } } ( n \omega _ { 0 } ) \} \left(\sin \left(\frac{n \pi}{2}\left(\rho_{2}-\rho_{1}\right)\right) \sin \left(n \pi\left(\frac{\rho_{2}+\rho_{1}}{2}-\rho_{p}\right)\right)\right.\right. \\
&+2 \sin \left(\frac{n \pi}{2}\left(\rho_{3}-\rho_{2}\right)\right) \sin \left(n \pi\left(\frac{\rho_{3}+\rho_{2}}{2}-\rho_{p}\right)\right) \\
&+\left.\left.\sin \left(\frac{n \pi}{2}\left(\rho_{4}-\rho_{3}\right)\right) \sin \left(n \pi\left(\frac{\rho_{4}+\rho_{3}}{2}-\rho_{p}\right)\right)\right)\right)
\end{aligned}
$$

Using the equation (4.24) and the constraints presented in (4.23), four oscillation conditions 
are obtained, shown in equation (4.25). In order to facilitate the lecture of these equations, it has been denoted as $C_{r e_{p}}$ and $C_{i m_{p}}$ the coefficients that multiply the real and imaginary parts of $G_{o l}$ respectively for each value of $p$. These coefficients only depend on the values of $\rho_{p}$.

$$
\begin{aligned}
& \sum_{n_{\text {odd }}}^{\infty}\left(\frac{1}{n}\left(\Im\left\{G_{o l}\left(n \omega_{0}\right)\right\} C_{i m_{1}}-\Re\left\{G_{o l}\left(n \omega_{0}\right)\right\} C_{r e_{1}}\right)\right)-\frac{\pi}{4}=0 \\
& \sum_{n_{\text {odd }}}^{\infty}\left(\frac{1}{n}\left(\Im\left\{G_{o l}\left(n \omega_{0}\right)\right\} C_{i m_{2}}-\Re\left\{G_{o l}\left(n \omega_{0}\right)\right\} C_{r e_{2}}\right)\right)-\frac{\pi}{2}=0 \\
& \sum_{n_{\text {odd }}}^{\infty}\left(\frac{1}{n}\left(\Im\left\{G_{o l}\left(n \omega_{0}\right)\right\} C_{i m_{3}}-\Re\left\{G_{o l}\left(n \omega_{0}\right)\right\} C_{r e_{3}}\right)\right)-\frac{\pi}{4}=0 \\
& \sum_{n_{\text {odd }}}^{\infty}\left(\frac{1}{n}\left(\Im\left\{G_{o l}\left(n \omega_{0}\right)\right\} C_{i m_{4}}-\Re\left\{G_{o l}\left(n \omega_{0}\right)\right\} C_{r e_{4}}\right)\right)=0
\end{aligned}
$$

From equation (4.25) it can be seen that, unlike the case of conditions for $m=1$ given by equations (4.8), it is impossible to isolate the terms $\Re\left\{G_{o l}\left(j \omega_{o}\right)\right\}$ and $\Im\left\{G_{o l}\left(j \omega_{o}\right)\right\}$, thus a robustness measure as $M_{T}$ with direct visual representation in the Nyquist plane can not be obtained when $m>1$. Then, in order to compare the robustness against oscillations with different $m$ a more general robustness measure must be defined. To this aim, in the case of equation (4.25), the minimal Euclidean distance between the origin $(0,0,0,0)$ and the points with coordinates given by the left hand members when evaluated in $\left.\rho_{p} \in\right] 0,1[, p=1,2,3,4$ and $\omega \in\left[\frac{\omega_{c g}}{200}, \omega_{c g}\right]$ can be used. Denoting $R_{2}$ the minimal Euclidean distance for $m=2$, and $L_{p}$ the left hand members of equation (4.25) for each $p$, the expression for the computation of $R_{2}$ is:

$$
R_{2}=\min _{\omega, \rho_{1}, \cdots, \rho_{4}} \sqrt{\sum_{p=1}^{4} L_{p}\left(\omega, \rho_{1}, \rho_{2}, \rho_{3}, \rho_{4}\right)^{2}} .
$$

Taking into account that for any value of $m$ the conditions in (4.3) can be written as a system of equations similar to (4.25) but with $2 m$ equations, the general expression for $R_{m}$ is as follows:

$$
R_{m}=\min _{\omega, \rho_{1}, \cdots, \rho_{2 m}} \sqrt{\sum_{p=1}^{2 m} L_{p}\left(\omega, \rho_{1}, \cdots, \rho_{2 m}\right)^{2}} .
$$

When $R_{m}=0$, the oscillation requirements are fulfilled, and thus, oscillations with $m$ levels could appear. On the other hand, if $R_{m}>0, m$-leveled oscillation will not occur. Obviously, the complexity of the calculus and the computation time needed to obtain $R_{m}$ increases with the number of oscillation levels $m$ because more combinations of the time fractions $\rho_{p}$ appear than in the case of $m=1$.

Using $R_{m}$ it is possible to compare the robustness of a system to different m-leveled oscillations. With this aim, the cases of $R_{1}, R_{2}$ and $R_{3}$ have been obtained for the batch of 95 models presented in equation (4.22) with their respective controllers. These robustness measures have been represented in Figure 4.23. As it can be seen, for all these models the value of $R_{m}$ increases with $m$. This shows that in general, by avoiding oscillations for $m=1$ with the proposed tuning method, the system will not present steady-state oscillations for higher values of $m$. 


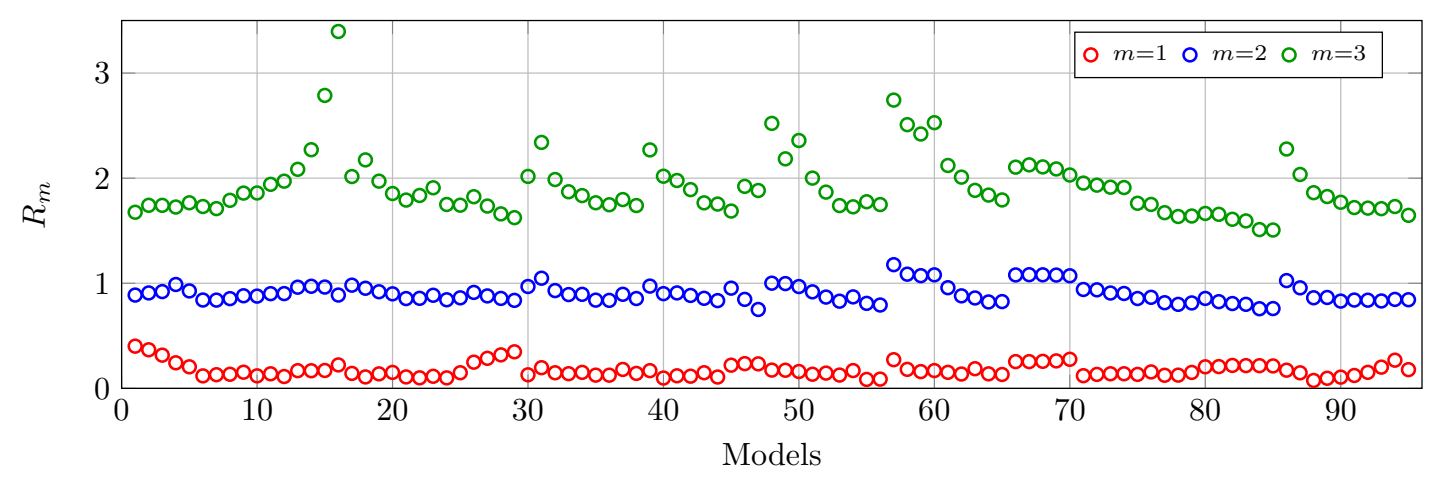

Figure 4.23: $R_{1}, R_{2}$ and $R_{3}$ for the batch of models with their respective controller obtained in the previous section.

\subsection{Tsypkin's method approach adapted for RQH sampling}

The Tsypkin methodology can be adapted for RQH sampling with minor changes. RQH sampling has been presented in Chapter 2, where an extensive analysis study has been presented, as in Chapter 3, where a tuning procedure has been proposed using as a base the Describing Function, which, as it has been shown in Example 10, may not be an adequate analysis tool for systems with low filtering capabilities.

Equation (4.5) remains valid to describe the output of the RQH sampler, which is essentially a ladder-like quantization, as in the SSOD case. However, the switching thresholds presented in equation (2.1) are different from those of the SSOD non-linearity, changing, therefore, the equality conditions, which are described by:

$$
E C_{p} \mid e\left(\rho_{p} \frac{T_{o}}{2}\right)= \begin{cases}\delta\left(p-\frac{1}{2}+\frac{h}{2 \delta}\right) & \text { for } p=1,2 \ldots m \\ \delta\left(2 m-p+\frac{1}{2}-\frac{h}{2 \delta}\right) & \text { for } p=m+1, \ldots 2 m\end{cases}
$$

and

$$
I C_{p} \mid \frac{d e\left(\rho_{p} \frac{T_{o}}{2}\right)}{d t}=\left\{\begin{array}{ll}
>0 & \text { for } p=1,2 \ldots m \\
<0 & \text { for } p=m+1, \ldots 2 m
\end{array} .\right.
$$

Based on the results presented in Section 4.6 it is reasonable to assume that by avoiding oscillations for $m=1$, the oscillations produced for $m>1$ are also avoided in the case of RQH sampling. Therefore the previous expressions, evaluated only for $m=1$ result in:

$$
E C_{p \mid m=1} \mid e\left(\rho_{p} \frac{T_{o}}{2}\right)=\left\{\begin{array}{cc}
\delta\left(\frac{1}{2}+\frac{h}{2 \delta}\right) & \text { for } p=1 \\
\delta\left(\frac{1}{2}-\frac{h}{2 \delta}\right) & \text { for } p=2
\end{array}\right.
$$


and

$$
I C_{p \mid m=1} \mid \frac{d e\left(\rho_{p} \frac{T_{o}}{2}\right)}{d t}=\left\{\begin{array}{ll}
>0 & \text { for } p=1 \\
<0 & \text { for } p=2
\end{array} .\right.
$$

Evaluating (4.5) in $p=1,2$ and substituting the result in (4.30) taking into account that by definition $\rho_{2 m}=1$ and renaming $\rho_{1}$ as $\rho$, the following equations are obtained:

$$
\begin{aligned}
& \sum_{n=1,3,5, \ldots}^{\infty} \frac{1}{n} \Re\left\{G_{o l}\left(j n \omega_{o}\right)\right\} \sin (n \pi \rho)+\frac{\pi}{4}=0 \\
& \sum_{n=1,3,5, \ldots}^{\infty} \frac{1}{n} \Im\left\{G_{o l}\left(j n \omega_{o}\right)\right\} \cos ^{2}\left(\frac{n \pi \rho}{2}\right)+\frac{\pi h}{8 \delta}=0
\end{aligned}
$$

that can be rewritten as:

$$
\begin{gathered}
\Re\left\{G_{o l}\left(j \omega_{o}\right)\right\}=-\frac{\frac{\pi}{4}+\sum_{n=3,5 . .}^{\infty} \frac{1}{n} \sin (n \pi \rho) \Re\left\{G_{o l}\left(j n \omega_{o}\right)\right\}}{\sin (\pi \rho)}, \\
\Im\left\{G_{o l}\left(j \omega_{o}\right)\right\}=-\frac{\frac{\pi h}{8 \delta}+\sum_{n=3,5 . .}^{\infty} \frac{1}{n} \cos ^{2}\left(\frac{n \pi \rho}{2}\right) \Im\left\{G_{o l}\left(j n \omega_{o}\right)\right\}}{\cos ^{2}\left(\frac{\pi \rho}{2}\right)} .
\end{gathered}
$$

As in the case of SSOD, the right hand members of the previous equations define a trajectory in the Nyquist plane that has been called Tsypkin branch, which is obtained for a given frequency $\omega_{o}$ and sweeping $\left.\rho \in\right] 0,1\left[\right.$. For a given frequency $\omega_{o}$ its Tsypkin branch is defined as:

$$
\left.B_{T}\left(\omega_{o}\right)=\left(-\frac{\frac{\pi}{4}+\sum_{n=3,5 . .}^{\infty} \frac{1}{n} \sin (n \pi \rho) \Re\left\{G_{o l}\left(j n \omega_{o}\right)\right\}}{\sin (\pi \rho)},-\frac{\frac{\pi h}{8 \delta}+\sum_{n=3,5 . .}^{\infty} \frac{1}{n} \cos ^{2}\left(\frac{n \pi \rho}{2}\right) \Im\left\{G_{o l}\left(j n \omega_{o}\right)\right\}}{\cos ^{2}\left(\frac{\pi \rho}{2}\right)}\right), \quad \forall \rho \in\right] 0,1[.
$$

Then, a limit cycle oscillation with frequency $\omega_{o}$ will take place if the Tsypkin branch for $\omega_{o}$ intercepts the point $\left(\Re\left\{G_{o l}\left(j \omega_{o}\right)\right\}, \Im\left\{G_{o l}\left(j \omega_{o}\right)\right\}\right)$ of the Nyquist diagram, that is:

$$
\left(\Re\left\{G_{o l}\left(j \omega_{o}\right)\right\}, \Im\left\{G_{o l}\left(j \omega_{o}\right)\right\}\right)=B_{T}\left(\omega_{o}\right) .
$$

The Tsypkin margin can be established for RQH sampling in the same way that for the SSOD, as the minimum euclidean distance between the Nyquist points $\left(\Re\left\{G_{o l}(\omega)\right\}, \Im\left\{G_{o l}(\omega)\right\}\right)$ 
and their respective Tsypkin branches obtained for the same frequency $\omega$, being its expression:

$$
M_{T}\left(\omega_{\text {min }}, \rho_{\text {min }}\right)=\sqrt{\left(\frac{\frac{\pi}{4}+\sum_{n_{\text {odd }}}^{\infty} \frac{1}{n} \sin \left(n \pi \rho_{\text {min }}\right) \Re\left\{G_{\text {ol }}\left(j n \omega_{\text {min }}\right)\right\}}{\sin \left(\pi \rho_{\text {min }}\right)}\right)^{2}+\left(\frac{\frac{\pi h}{8 \delta}+\sum_{n_{\text {odd }}}^{\infty} \frac{1}{n} \cos ^{2}\left(\frac{n \pi \rho_{\text {min }}}{2}\right) \Im\left\{G_{\text {ol }}\left(j n \omega_{\text {min }}\right)\right\}}{\cos ^{2}\left(\frac{\pi \rho_{\text {min }}}{2}\right)}\right)^{2}},
$$

being $\omega_{\min }$ and $\rho_{\min }$ the values of $\omega$ and $\rho$ for which the minimum distance is obtained.

The Tsypkin margin $M_{T}$ for a RQH sampling shares a lot of similitudes with its homologue for SSOD, being the unique difference in the second term inside the root expression of equation (4.36). In this term, the ratio $h / \delta$ appears in the numerator of the expression, which is the main characteristic to take into account for RQH samplers.

Despite the similitudes with Tsypkin's margin for SSOD, it must be highlighted that the frequency $\omega_{\min }$ and the semiperiod time fractions $\rho_{\text {min }}$ which make one margin minimum differ from those that would make the margin minimum for the other sampler. Hence, the variability on $M_{T}$ is propitiated by the change of the ratio $h / \delta$, but it is not a direct substitution in the equation (4.36).

To introduce this concept and prove the validity of Tsypkin's margin for RQH samplers the following example is introduced.

Example 17. Consider for this example the process presented in the Example 10. Consider also the controller tuned with SRB method presented in that example. Several samplers with different ratios $h / \delta$ will be tested, specifically, the chosen samplers will be $h / \delta=[1,3 / 4,1 / 2,1 / 4]$, including, therefore, the SSOD case $(h=\delta)$.

As it is known from Example 10, the system will present limit cycle oscillations when tested under a SSOD sampling scheme. In order to determine the robustness of the systems when the sampler is replaced the Tsypkin margin presented in equation (4.36) has been used. As expected the SSOD case presents $M_{T}=0$ and the same result is obtained for the sampler with $h / \delta=3 / 4$. However, a value of $M_{T}=0.29$ and $M_{T}=0.3$ is obtained for the samplers with $h / \delta=1 / 2$ and $h / \delta=1 / 4$ respectively, avoiding limit cycle oscillations.

The obtained margins have been presented graphically in Figure 4.24, where it can be seen the distance between the open-loop transfer function with its respective minimum Tsypkin branch, resulting in $h / \delta=1$ and $h / \delta=3 / 4$ in an intersection. As it can be seen, the frequencies in which the minimum Tsypkin branch is obtained differ from one case to another, highlighting the fact that changing the sampler does not imply a mere substitution of the ratio $h / \delta$ in equation (4.36).

These systems have been tested in simulation against a disturbance step-like change of magnitude 2.5. The individual parameters $h$ and $\delta$ have been chosen to accomplish a steady-state error equal to 0.1 using equation (2.2). The temporal response for the four cases can be observed in Figure 4.25, where it can be seen that for those cases where $M_{T}=0$ the systems present limit cycle oscillations and when $M_{T}>0$ the systems do not oscillate, corroborating, therefore, the theoretical results obtained with the robustness measure. 

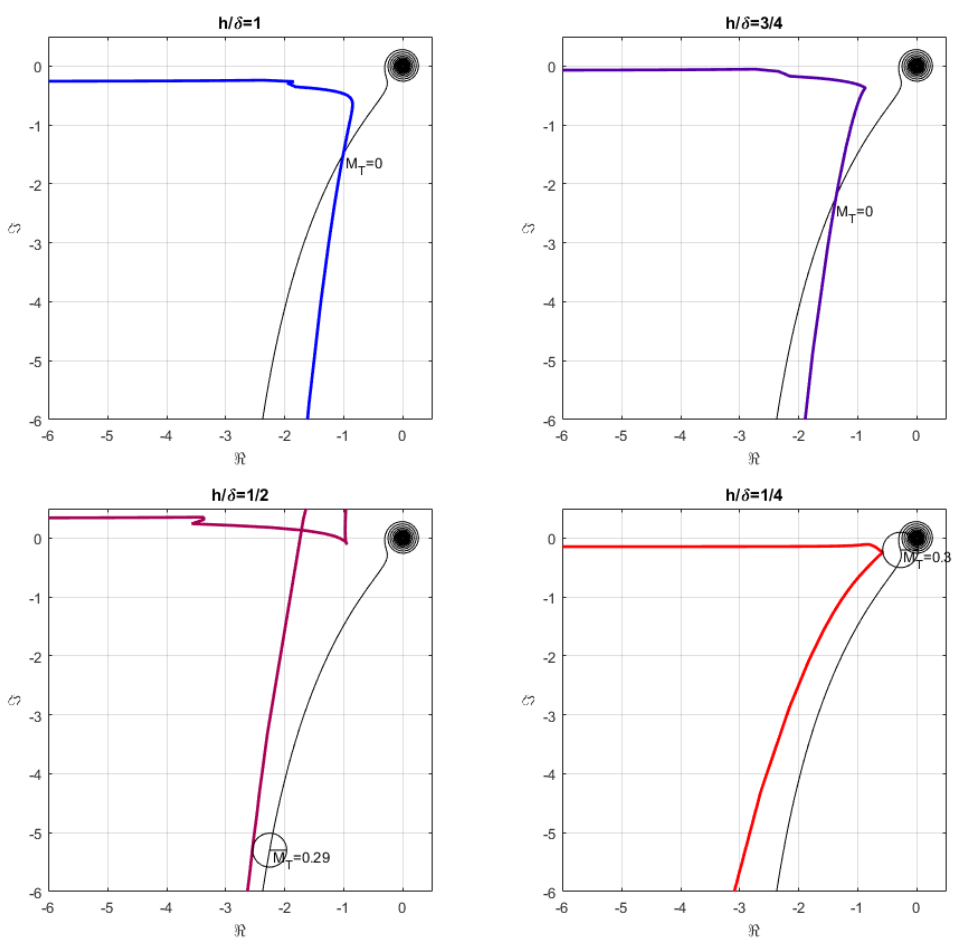

Figure 4.24: Nyquist plot of the open-loop transfer function with all the controllers and their minimum Tsypkin branch.
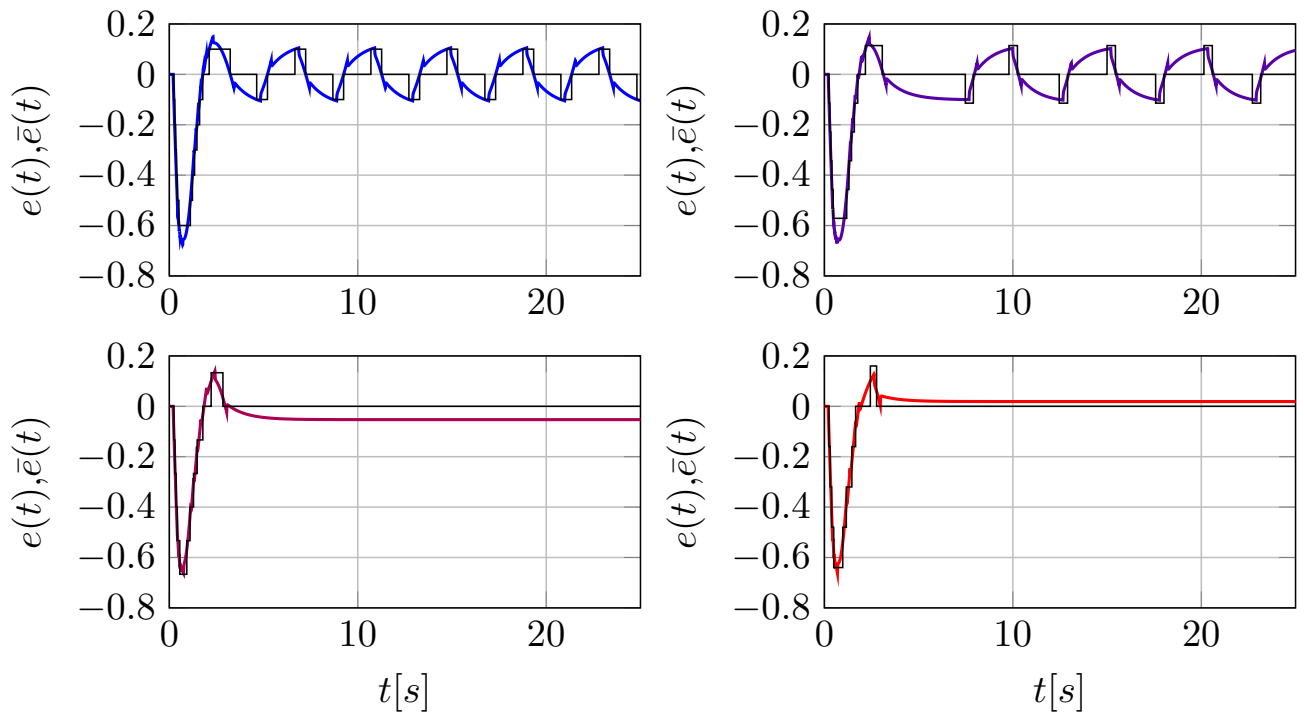

Figure 4.25: Temporal response of the error signal $e(t)$ and sampled error signal $\bar{e}(t)$ against disturbance step change of magnitude 2.5 for the four samplers. (Same disposition and colors as in Figure 4.24) 


\subsection{Conclusions}

The chapter presents a PID tuning procedure for FOPTD systems with Symmetric-Send-OnDelta sampling, which allows minimizing the disturbance IAE while fulfilling constraints on the robustness against oscillations and on the control action changes.

The robustness to avoid limit cycle oscillations due to the SSOD sampling has been taken into account by defining a new robustness measure, $M_{T}$, based on Tsypkin's method, which does not require the system to filter out the high order harmonics (as it is required by methods based on the Describing Function). Therefore, the proposed approach is valid for systems with insufficient filtering characteristics (where the describing function can not be applied). The analysis method is shown to be applicable with minor changes to the analysis of loops with a Regular Quantization with Hysteresis sampling strategy.

A set of precomputed controllers has been obtained for dimensionless FOPTD models with different values of $L / \tau$, using a grid on phase margin and on derivative filter parameter $N$. For each controller in the set, the value of $M_{T}$ and $C(\infty)$ has been computed. The tuning procedure consists in selecting from this set the controller that minimizes the $I A E$ and fulfills the required $M_{T}$ and $C(\infty)$ constraints.

In order to simplify the application of the tuning procedure, a Java application has been developed (freely available at https://sites.google.com/a/uji.es/freepidtools/ssodTsypkinPid), which computes the controller parameters for the required $M_{T}$ and $C(\infty)$. If the system model is not FOPTD, the applicacion first computes a FOPTD approximation, and calculates de PID controller using that approximated model. Alternatively to this application, a table for tuning controllers with $M_{T}>0.2$ and restrictions on $C(\infty)$ is also provided.

An extensive study with a batch of models has shown that when the system is not FOPTD, the controller obtained with the FOPTD approximation results in higher values of $M_{T}$ than initially required, therefore, the procedure guarantees the robustness to limit cycle oscillations for any system model.

The tuning procedure shows that the derivative filter coefficient $N$ is a crucial tuning parameter for SSOD PID, because it allows reaching a reasonable compromise between performance (in terms of $I A E$ for example) and control action bumps due to the SSOD sampling. 


\title{
Characterization of limit cycle oscillations induced by Fixed Threshold Samplers
}

\begin{abstract}
In this chapter, a generalized study of the limit cycle oscillations induced by any kind of sampler with multilevel fixed thresholds is presented. The Fixed Threshold Samplers (FTS) are characterized by a series of parameters, which, when selected properly, allow obtaining some of the most used forms of quantization. Because of some sampler characteristics, the obtained limit cycle oscillations can present a bias, therefore, to characterize them the Dual Input Describing Function (DIDF) method is used. The obtained DIDF is analyzed revealing some interesting properties allowing to simplify the robustness analysis. Guidelines about how to perform the robustness analysis are given, showing their application through some study cases.
\end{abstract}

\subsection{Introduction}

In recent days, Event-Based Control (EBC) is becoming a more and more popular control alternative. This change of paradigm is motivated by the apparition of a new industrial framework named Industry 4.0. This new communication benchmark requires efficient data flow through the industrial networks, fostering the implementation of EBC algorithms.

Several authors have already applied the EBC to well known control algorithms, notably to the PID algorithm, which constitutes one of the most simple and reliable algorithms, and therefore, it is placed among the most used in the industry [62]. In addition, its role within the context of Industry 4.0 has been brought forward in [39].

One of the first works referring to Event-Based PID control algorithm was Årzén's contribution [3], in which it was shown how this algorithm could be used as a tool to reduce the CPU 
usage to perform the control of a process without affecting significantly its performance. In that work some key aspects about EBC were identified, specially, the error involving the calculation of integral and derivative terms when the elapsed time between samples increases.

Subsequently, some works have been addressed to resolve these problems, mainly regarding the integral time calculation. In that sense, the contributions made by Durand $[22,23]$ and Vasyutynskyy [76, 77] must be highlighted. Other works like [41] show the validity of this kind of algorithms when implemented with the standard IEC 61499 [80], which supports the implementation of distributed control systems.

As important as the control algorithm is the sampling strategy used to regulate them, which is in charge of generating events whenever significant changes are detected in the state of the controlled system. Among the most used strategies the Fixed Threshold Samplers (FTS) can be found. Some examples of this kind of sampling strategies are the Regular Quantization (RQ), the Symmetric-Send-On-Delta SSOD [10] or the Regular Quantization with Hysteresis (RQH) [42] which represents an intermediate case.

A lot of works have been addressed considering these strategies into the loop. There can be found works in which they have been used for identification purposes [70,64, 65], but more importantly, for the control of processes $[9,49,59]$ and, for a correct performance, several tuning rules and procedures have been proposed $[11,56,44,63]$.

Most of the aforementioned works deal with a drawback of these kinds of FTS, which is that they can induce limit cycle oscillations. Under the perspective of EBC these oscillations degrade the loop performance, accelerate the wear out of actuators and overload the communication networks with unnecessary events, however, regarding these oscillations under the principles of relay control, an oscillatory state can be the expected result.

In this chapter, the limit cycle oscillations induced by any kind of FTS are characterized. To that end, a variant of the Describing Function (DF) known as Dual Input Describing Function (DIDF) [78] is used as analysis tool. To perform the study the FTS is parametrized and the general DIDF is obtained.

The chapter is organized as follows. Section 5.2 presents the generalized FTS, defining its characteristic parameters, and shows how other samplers can be obtained by choosing the correct parameters. Section 5.3 presents the DIDF approach and some preliminary properties necessary to develop further analysis. Section 5.4 studies the behavior of different type of process under FTS loops and how to analyze them. Finally, the conclusions about this chapter are drawn in Section 5.5.

\subsection{Problem statement}

The choice of a proper sampler, or the task to adequate it to the given necessities, can be challenging, specially considering that the mere fact of quantifying can induce limit cycle oscillations. 


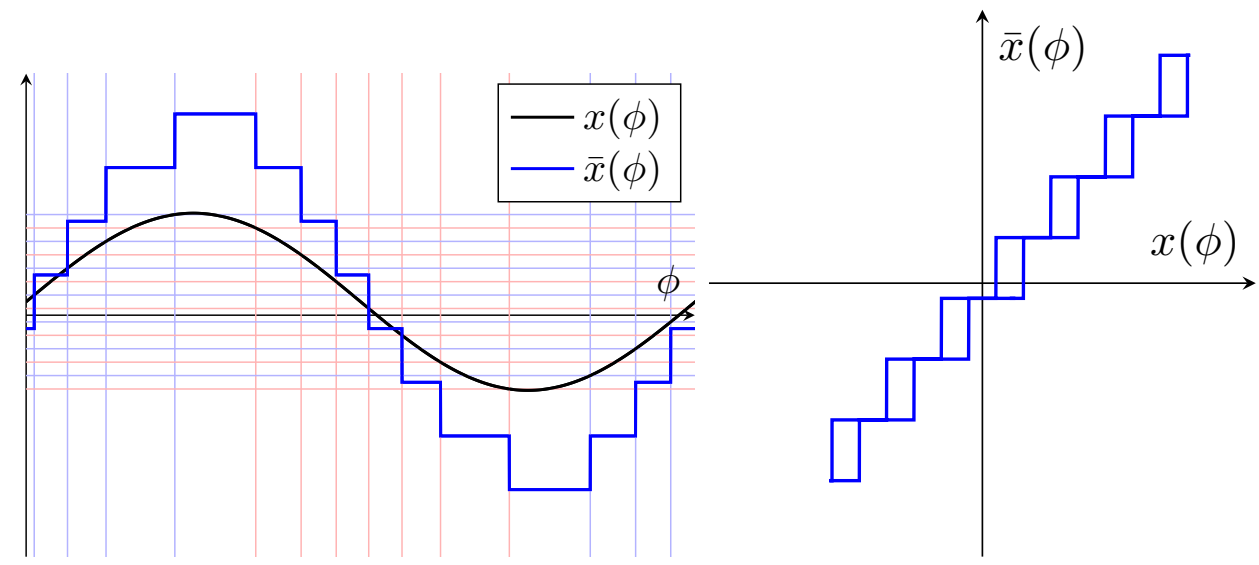

Figure 5.1: On the left image, sinusoidal oscillation sampled with a given FTS. On the right: input-output relationship of the chosen FTS (parameters: $\Delta \delta / \delta=1 / 2, \Delta \epsilon / \epsilon=1 / 4, \epsilon / \delta=2$, $h / \delta=1 / 2$, explained below).

In addition, as the complexity of the sampler increases, the resulting oscillations can become difficult to predict. For example consider the oscillation presented in Figure 5.1 produced by a given FTS. In this figure, it can be observed a huge difference between the input signal $x(\phi)$ and the output of the FTS $\bar{x}(\phi)$. Among the main differences it can be seen that the quantization step is higher than the increase in the signal, the switching thresholds do not start at the origin, the output of the FTS is not symmetric and the number of levels crossed differs between upwards (four) and downwards (three) direction.

All this characteristics can be parametrized by regarding the relationship between input $(x)$ and output $(\bar{x})$ of a general FTS, which is summarized graphically in Figure 5.2. In this figure, it can be seen all the parameters that characterize a FTS, namely, the detection width $\delta$, the detection hysteresis $h$, the detection asymmetry $\Delta \delta$, the output quantification $\epsilon$ and the output asymmetry $\Delta \epsilon$. The asymmetries have been measured with regard to the center of the rectangle $\delta \times \epsilon$ and the hysteresis $h$ is vertically centered on that rectangle. It is considered without loss of generalization that $\Delta \epsilon \in[-\epsilon / 2, \epsilon / 2]$ and $\Delta \delta \in[-\delta / 2, \delta / 2]$.

These parameters already prognosticate the behavior of the sampler and the system. For example, the sampler will contain a dead zone only if $\Delta \epsilon= \pm \epsilon / 2$, it will present a symmetric output only if $\Delta \delta= \pm \delta / 2$ or it will present a certain amplification or attenuation depending on the relationship between $\epsilon$ and $\delta$. Additionally, it is worth noticing that by choosing a certain combination of parameters the most common FTS samplers traditionally studied can also be found. A summary with some of them can be found in Table 5.1. Obviously, this table only presents some cases of multileveled samplers, there exist many other options that can be obtained with the appropriate configuration. 
Table 5.1: Summary of samplers and their parameter configuration.

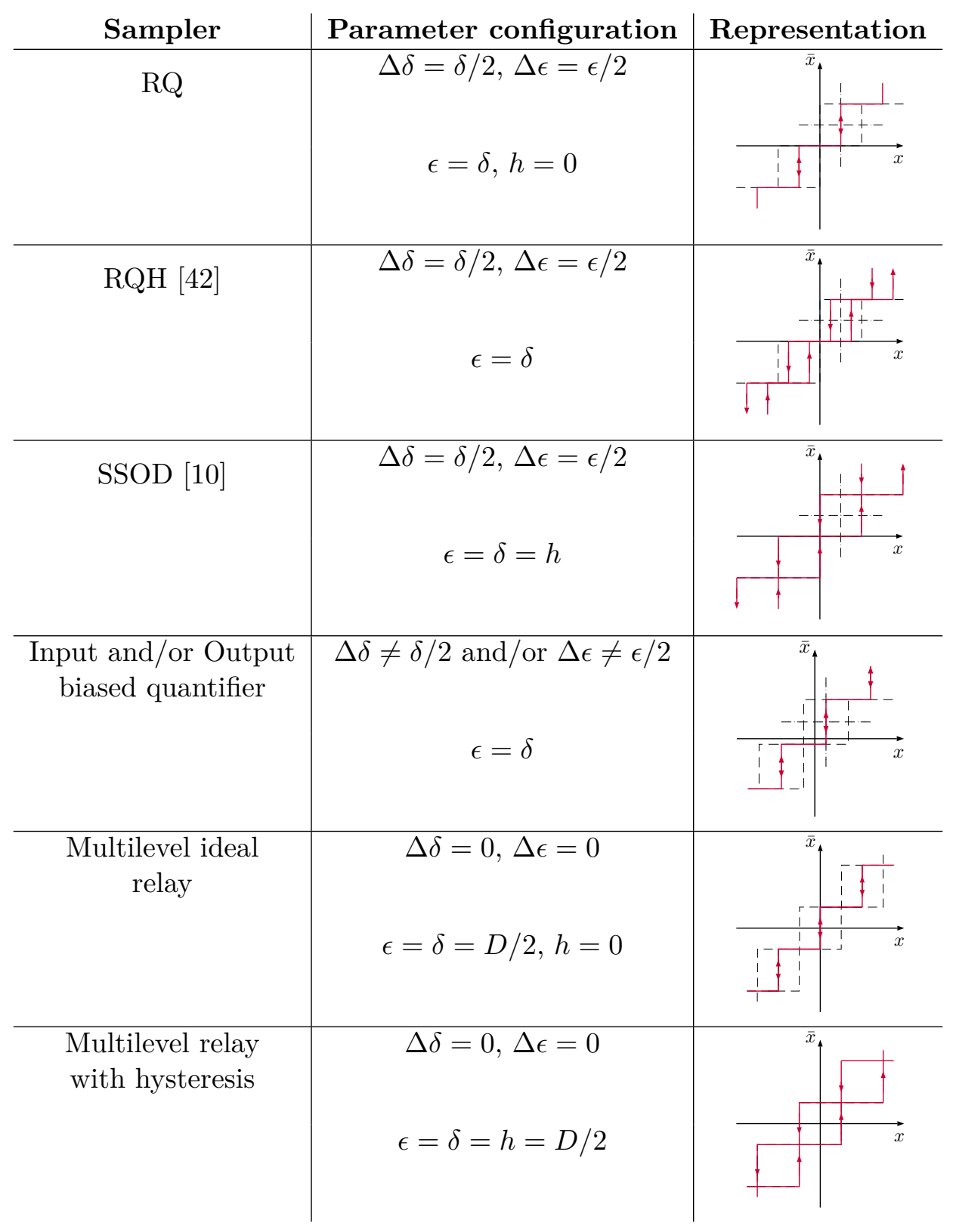




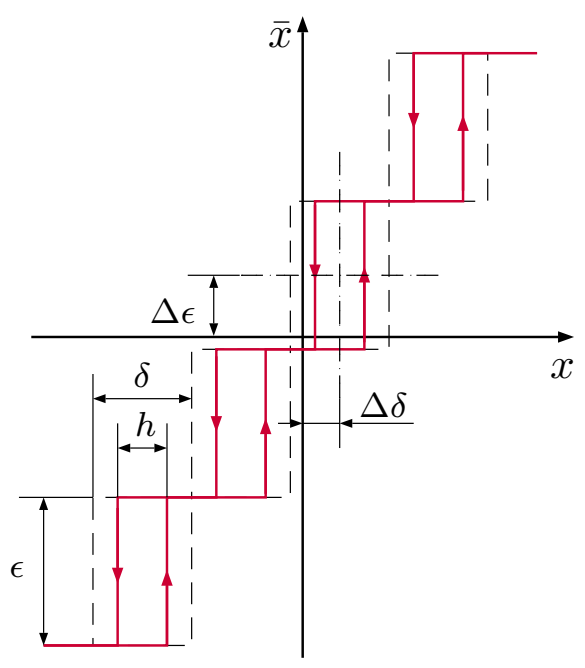

Figure 5.2: Relationship between input and output of a generic FTS.

\subsection{DIDF approach}

As it has been previously commented, one of the most important phenomena when using a FTS is that they can induce limit cycle oscillations. To study this kind of non-linear behavior there exist several tools, among them, the Describing Function approach excels due to its ease of use and accuracy in the obtained results $[60,58]$.

The generic FTS under study includes, depending on the chosen parameters, a wide variety of commonly used samplers, SSOD [10], RQH [42] or an RQ sampling among them, as it has been shown in the precedent section. Therefore, by addressing an analysis considering the most generic case a wide variety of samplers can be characterized. Considering the parameters previously described, the relationship between the input signal to the sampler $x(t)$ and its output $\bar{x}(t)$ is defined by:

$\bar{x}(t)=\left\{\begin{array}{lll}\Delta \epsilon-\frac{\epsilon}{2}+\epsilon(i+1) & \text { if } \quad x(t) \geq \Delta \delta+\frac{h}{2}+\delta i \quad \text { and } \quad \bar{x}\left(t^{-}\right)=\Delta \epsilon-\frac{\epsilon}{2}+\epsilon i, \quad i \in \mathbb{Z} \\ \Delta \epsilon-\frac{\epsilon}{2}+\epsilon(i-1) & \text { if } \quad x(t) \leq \Delta \delta-\frac{h}{2}+\delta(i-1) \quad \text { and } \quad \bar{x}\left(t^{-}\right)=\Delta \epsilon-\frac{\epsilon}{2}+\epsilon i \\ \Delta \epsilon-\frac{\epsilon}{2}+\epsilon i & \text { if } \quad x(t) \in\left[\Delta \delta-\frac{h}{2}+\delta(i-1), \Delta \delta+\frac{h}{2}+\delta i\right] \quad \text { and } \quad \bar{x}\left(t^{-}\right)=\Delta \epsilon-\frac{\epsilon}{2}+\epsilon i\end{array}\right.$

To apply the DF technique consider a generic control loop like the one presented in Figure 5.3. Consider that the controller $C(j \omega)$ and the process $G(j \omega)$ can be grouped in a single block $G_{o l}(j \omega)=C(j \omega) G(j \omega)$ representing the open-loop transfer function which gathers all the linear behavior on the loop.

Because of the inherent generalization induced by the FTS, there may exist some possible bias in the output of the sampler which may not be attenuated through the loop and, therefore, 


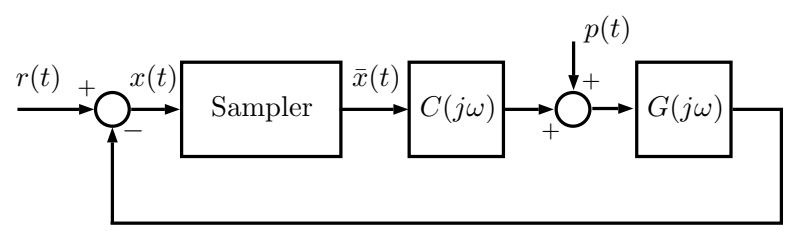

Figure 5.3: Generic loop structure that allows the DF technique application.

it must be considered at the input of the non-linearity. Thus, in this type of oscillatory steadystate the input to the sampler corresponds to the form $x(t)=A \sin (\omega t)+B$. Therefore, to include in the study the cases where a bias appears, a variant of the DF is used called Dual Input Describing Function (DIDF), which studies the sustainability of the oscillation and the bias.

Applying the DIDF principles, it is known that the condition for the maintainability of an unattenuated oscillation, called limit cycle, in this kind of systems is given by:

$$
G_{o l}(j \omega)=-\frac{1}{\mathcal{N}_{A}}, \quad \forall \omega
$$

where $\mathcal{N}_{A}$ is the describing function of the non-linear element related to the dynamic behavior. This condition can be interpreted graphically as: if it exists intersection between $G_{o l}(j \omega)$ and the inverse negative of $\mathcal{N}_{A}$ in the Nyquist diagram, a limit cycle can occur.

In addition to the condition for the dynamic part presented in equation (5.1), the sustainability of the bias through the loop should also be studied. The conditions involving the bias maintenance were presented in [25] and, as in the oscillation maintenance, also depend on the linear part of the process under study. However, those conditions did not take into account the effect of the disturbance and reference input, which were considered to be $r(t)=0$ and $p(t)=0$, therefore, they have to be adjusted to take into account these signals.

Consider the loop structure presented in Figure 5.3 and assume reference and disturbance inputs to be step-like signals. Assume also that a biased oscillation appears, so that the bias on $x(t)$ is $B$. Then, considering only the non-oscillatory parts of the elements in the loop:

$$
\begin{aligned}
x(\infty) & =r(\infty)-y(\infty) \\
B & =r(\infty)-G(0)\left[p(\infty)+B \mathcal{N}_{B} C(0)\right],
\end{aligned}
$$

where $r(\infty)$ and $p(\infty)$ are the magnitude of the changes in reference and disturbance signals, $G(0)$ and $C(0)$ the steady-state gains of the process and the open-loop transfer function, $B$ the bias and $\mathcal{N}_{B}$ the describing function for the maintenance of the bias. Arranging the previous expression, the condition for the maintenance of the bias is obtained:

$$
r(\infty)-p(\infty) G(0)=B\left(1+\mathcal{N}_{B} G_{o l}(0)\right),
$$

where $G_{o l}(0)$ is the steady-state gain of the open-loop transfer function. This expression depends on the form of $G(s)$ and $C(s)$ since it depends on the steady-state gain of both. For several 
combinations between the form of $G(s)$ and $C(s)$, expression (5.2) has been further developed in Table B.1 of Appendix B.3.

The DIDF of the general FTS involving the bias maintenance and the dynamic oscillations are given respectively by the following equations (see Appendix B.2):

$$
\begin{aligned}
\mathcal{N}_{B}= & \frac{2 \Delta \epsilon+\epsilon\left(m_{\text {sup }}-m_{\text {inf }}+2 i_{0}-1\right)}{2 B} \\
& -\frac{\epsilon}{2 \pi B} \sum_{k=-m_{\text {inf }}}^{m_{\text {sup }}-1}\left[\arcsin \left(\frac{1}{A}\left(\Delta \delta+\frac{h}{2}-B+\delta\left(k+i_{0}\right)\right)\right)+\arcsin \left(\frac{1}{A}\left(\Delta \delta-\frac{h}{2}-B+\delta\left(k+i_{0}\right)\right)\right)\right]
\end{aligned}
$$

and

$$
\begin{aligned}
\mathcal{N}_{A}= & \frac{\epsilon}{\pi A} \sum_{k=-m_{\text {inf }}}^{m_{\text {sup }}-1}\left[\sqrt{1-\left(\frac{1}{A}\left(\Delta \delta+\frac{h}{2}-B+\delta\left(k+i_{0}\right)\right)\right)^{2}}+\sqrt{1-\left(\frac{1}{A}\left(\Delta \delta-\frac{h}{2}-B+\delta\left(k+i_{0}\right)\right)\right)^{2}}\right] \\
& -j \frac{\epsilon h}{\pi A^{2}}\left[m_{\text {sup }}+m_{\text {inf }}\right]
\end{aligned}
$$

where $i_{0}$ is the initial or base level, around which the oscillations are produced. The oscillations can have a number of $m_{\text {sup }}$ levels crossed in upwards direction and $m_{\text {inf }}$ in downwards direction from $i_{0}$. These parameters are calculated as follows:

$$
m_{\text {sup }}=\left\lfloor\frac{A+B-\Delta \delta-h / 2-\delta i_{0}}{\delta}\right\rfloor+1, \quad m_{\text {inf }}=-\left\lfloor\frac{-A+B-\Delta \delta+h / 2-\delta i_{0}}{\delta}\right\rfloor-1
$$

and:

$$
i_{0}=\left\{\begin{array}{l}
\left\lfloor\frac{B-\Delta \delta-h / 2}{\delta}\right\rfloor+1 \quad \text { if } \quad B \geq 0 \\
\left\lfloor\frac{B-\Delta \delta+h / 2}{\delta}\right\rfloor \quad \text { if } \quad B<0
\end{array} .\right.
$$

The DF for the bias and for the sinusoidal part can be expressed with a series of meaningful dimensionless ratios, namely, $\epsilon / A, \epsilon / B, \delta / A, \delta / B, \Delta \epsilon / \epsilon, \Delta \delta / \delta$ and $h / \delta$. The ratios that describe the sampler are bounded: $\Delta \epsilon / \epsilon \in[-1 / 2,1 / 2], \Delta \delta / \delta \in[-1 / 2,1 / 2]$ and it will only be considered $h / \delta \in[0,1]$ even though it can take a wider range. The other ratios involve the input signal and, therefore, they cannot be bounded.

By substituting in the previous expressions the parameter configurations presented in Table 5.1, the dynamic describing function $\left(\mathcal{N}_{A}\right)$ considering $B=0$ can be found for the RQ [58], RQH [42] or SSOD [60] for example.

\subsubsection{Characteristics of the dynamic part of the DIDF}

Due to the generality of the expression presented above and the number of parameters involved, the analysis study will result in a complex research. Therefore, to bound this study, $\mathcal{N}_{A}$ is 

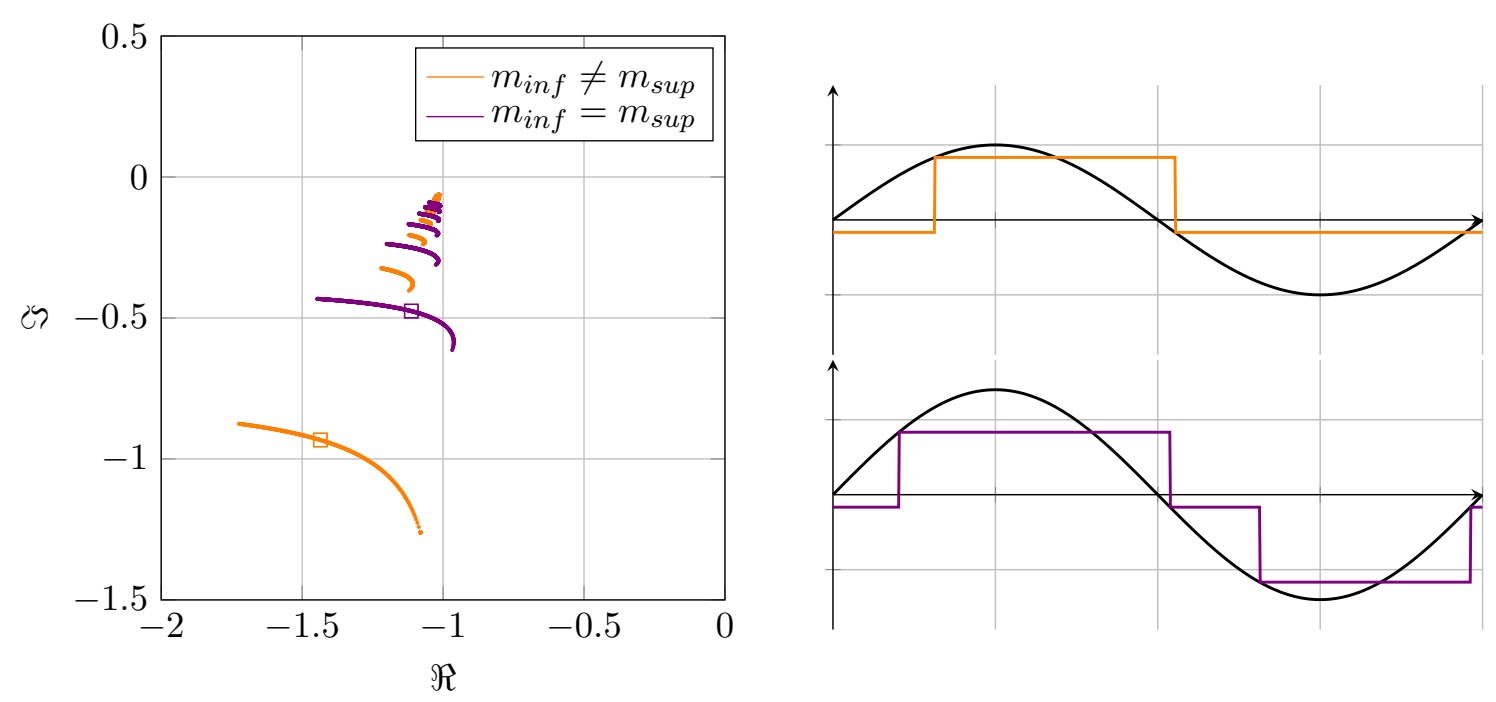

Figure 5.4: $\mathcal{N}_{A}$ of a sampler with two series of traces regarding the levels crossed in each direction (Parameters used: $\Delta \epsilon / \epsilon=\Delta \delta / \delta=1 / 3, \epsilon=\delta=h$ ).

evaluated analyzing its behavior under certain conditions. This evaluation leads to a better comprehension of the behavior of $\mathcal{N}_{A}$ and to a significant reduction of the analysis study effort.

The first behavior has been already presented in Figure 5.1, where the oscillations present different number of levels crossed upwards and downwards from $i_{0}$, i.e. $m_{\text {sup }} \neq m_{\text {inf }}$. Nevertheless, the same sampler can provide oscillations with the same number of levels crossed upwards and downwards, $m_{\text {sup }}=m_{\text {inf }}$, depending on the amplitude $A$ of the oscillation. This behavior is reflected in the dynamic part of the DF by presenting two alternating "series" of bands, one where $m_{\text {sup }} \neq m_{\text {inf }}$ and another with $m_{\text {sup }}=m_{\text {inf }}$ alternatively. That can also be seen evaluating the expressions in (5.5) for a range of values of $A$.

On the left image of Figure 5.4, the inverse negative of the dynamic DF $\mathcal{N}_{A}$ (see equation (5.4)) of a sampler with $\Delta \epsilon / \epsilon=\Delta \delta / \delta=1 / 3$ and $\epsilon=\delta=h$ and without bias has been represented. The presented traces are grouped in two series that have been plotted in different colors. In that image, two squares, one in each series, have been marked, which correspond to the oscillation presented on the images on the right. As it can be seen, the orange oscillation only has one upper level and no levels are crossed downwards from the initial level, whereas the violet oscillation has the same number of levels crossed in both directions.

Despite the ratio $\epsilon / \delta$ not being a ratio used to define the DF, as it can be seen from equations (5.4) and (5.3), it has a great influence on the placement of the DF traces. This ratio describes the relationship between the detection and the output thresholds of the sampler, therefore, it has the same behavior as a gain. Thus, the DF traces present a radial shrink when this ratio increases and a radial expansion when it decreases. Therefore, this parameter is of great influence to the robustness of the loop since it restricts the frequency response of the linear elements. This radial variation of $-1 / \mathcal{N}_{A}$ has been represented in Figure 5.5 where the inverse 


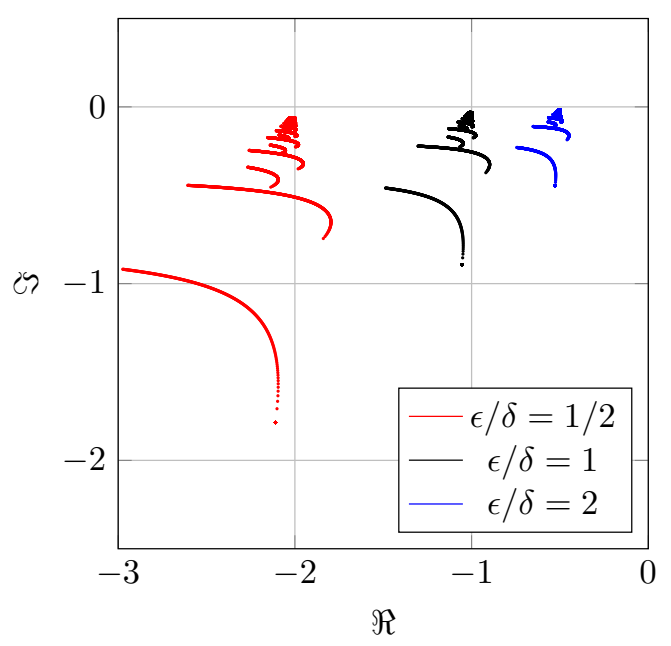

Figure 5.5: Radial variance of $-1 / \mathcal{N}_{A}$ with the ratio $\epsilon / \delta$ (Parameters used: $\Delta \epsilon / \epsilon=1 / 4, \Delta \delta / \delta=1 / 3$ and $h / \delta=1 / 2)$.

negative of a sampler with $\Delta \epsilon / \epsilon=1 / 4, \Delta \delta / \delta=1 / 3$ and $h / \delta=1 / 2$ has been presented using three different ratios $\epsilon / \delta$.

Another parameter which plays a paramount role is the relative hysteresis, presented by the ratio $h / \delta$, which prevents the generation of unnecessary events due to the presence of noise in the sampled signal [42]. In addition, regarding this parameter from the non-linear analysis point of view, it makes the sampler take into consideration from where does the sampled signal come, i.e. it adds a memory effect. As it was stated in [25], non-linearities with memory present an imaginary part in the inverse negative of their DF and those without memory only have a real part. This fact is corroborated in Figure 5.6, in which a sampler with $\Delta \epsilon / \epsilon=1 / 5, \Delta \delta / \delta=1 / 2$ and $\epsilon / \delta=0.75$ has been tested with three different levels of relative hysteresis, showing that the traces fold gradually towards the real axis as the hysteresis is reduced.

In addition to the influence of the parameters of the sampler, in those cases where a bias is present, i.e. the input to the non-linearity is of the form $x(t)=A \sin (\omega t)+B$ with $B \neq 0$, it plays an important role on the shape of the DF under study.

The presence of this bias can change completely the behavior expected of a given sampler. For example, imagine any FTS with a deadband (SSOD, RQH, etc.). If the loop contains any element that accomplishes a bias $B$ such as $\Delta \delta=B$, then the behavior of the FTS will not correspond to a deadband, but it will be more similar, for example, to a multilevel relay, i.e. a sampler with no deadband. This fact is logical because the value of the bias $B$ compensates the asymmetry $\Delta \delta$, and therefore the same behavior is expected. Traduced to the analysis of $\mathcal{N}_{A}$, this fact implies that for a given sampler in which $\Delta \delta=B$ a straight line that reaches the imaginary axis will appear, as it appears for other non-linearities without deadband, for example, the ideal relay. This effect of the asymmetry compensation with the bias is illustrated in Figure 5.7, where the parameters of the SSOD are used and it can be seen how an horizontal line that reaches the imaginary axes appears. 


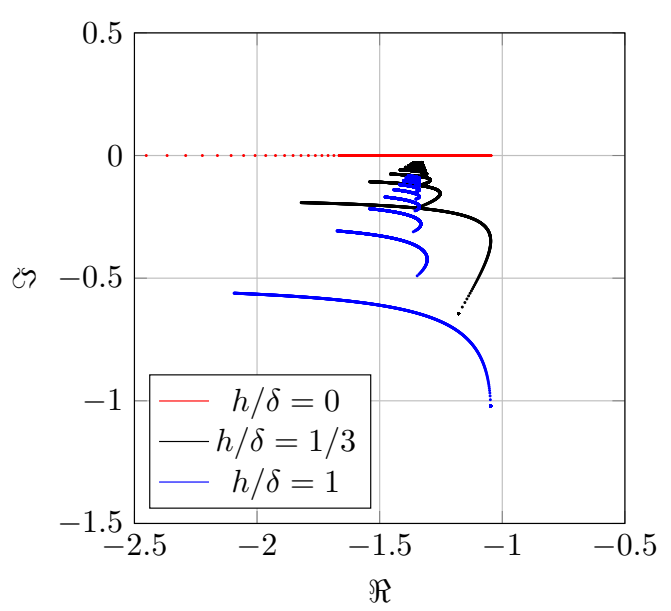

Figure 5.6: Effect of the hysteresis on $-1 / \mathcal{N}_{A}$ (Parameters used: $\Delta \epsilon / \epsilon=1 / 5, \Delta \delta / \delta=1 / 2$ and $\epsilon / \delta=0.75)$.

The apparition of a bias can also propitiate a lag between the upwards and downwards crossed levels. This can be seen from equations in (5.5): having a given sampler makes the number of levels vary differently when sweeping $A$ for different values of $B$. That is to say that the apparition of a bias is equivalent to an asymmetry.

For the analysis study it can be very concerning this big variance of the traces of $-1 / \mathcal{N}_{A}$ with the bias $B$. Nevertheless, it has been observed that the placement of these traces is periodically repeated with the change of $B$. Concretely, the placement of the traces will be the same for:

$$
\frac{-1}{\mathcal{N}_{A}(B)}=\frac{-1}{\mathcal{N}_{A}\left(B^{\prime}\right)}
$$

where $B^{\prime}=B+k \delta$ and $k \in \mathbb{Z}$. This result is logical because it only represents a shift, and it can be observable from the expressions of $\mathcal{N}_{A}$ and of the levels crossed $m_{\text {sup }}$ and $m_{\text {inf }}$ where the ratio $\delta / B$ and the levels are presented together.

Besides this periodicity, it has also been observed that $-1 / \mathcal{N}_{A}$ presents the same traces for given variations of $B$ lesser than $\delta$. Concretely, the obtained traces are the same if $B$ changes with regard to some symmetry axes, being these symmetry axes the extreme and the center of the square $\delta \times \epsilon$. Therefore, the obtained traces will be the same for $B=\Delta \delta-\delta / 2+\alpha$ and for $B^{\prime}=\Delta \delta-\delta / 2-\alpha, \alpha \in \Re$. And regarding the other symmetry axis, the same traces will be obtained considering $B=\Delta \delta+\beta$ and $B^{\prime}=\Delta \delta-\beta, \beta \in \Re$.

With regard to $\mathcal{N}_{B}$, no generic properties can be extracted from its study as for the case of $\mathcal{N}_{A}$. The application of the part of the DIDF related to the bias will be treated deeply in the next section. 


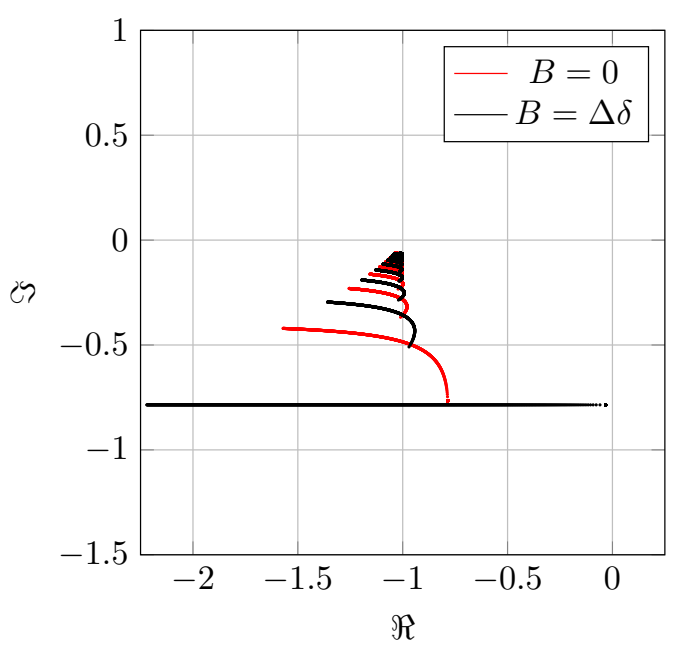

Figure 5.7: Asymmetry compensation with the bias $B$ and its effect on $-1 / \mathcal{N}_{A}$ (Parameters used: $\Delta \epsilon / \epsilon=1 / 2, \Delta \delta / \delta=1 / 2$ and $\epsilon=\delta=h)$

\subsection{Oscillation conditions study}

The study will pursue different objectives depending on the studied sampler, its application and the process involved. For example, it may be desired that a given system presents an oscillatory response around a given set point with controlled oscillations or that a system presents a robust behavior against these limit cycle oscillations that the DIDF describes.

To perform the analysis it is necessary to know the bias and if it is not attenuated through the loop even though the exact bias is not known a priori. However, it can be estimated by considering that the changes produced on the loop, whether their source is the disturbance or the reference, are big enough with regard to the detection threshold $\delta$. In that case, the effect of the sampler can be substituted by $\epsilon / \delta$, which is the slope of input-output relationship (see Figure 5.2). Then, an estimation of the bias, which will be referred to as bias central value $B_{c}$, can be obtained from expression (5.2):

$$
B_{c}=\frac{r(\infty)-p(\infty) G(0)}{1+\epsilon / \delta G_{o l}(0)} .
$$

From this central value, a set of candidate biases with amplitude $\delta$ will be considered defined as $B_{s} \in\left[B_{c}-\delta / 2, B_{c}+\delta / 2\right]$. The amplitude of the set is chosen to be $\delta$ due to the symmetry property and periodicity of the dynamic part explained above.

Then, using the maintenance condition of the bias, which will be one of the presented in Table B.1 depending on the elements of the loop, for the values in $B_{s}$, pairs $\{A, B\}$ which are candidate to present limit cycle oscillations are obtained for the given process, controller and sampler. Nevertheless, the pairs $\{A, B\}$ must be evaluated to determine whether they conduce to an unattenuated propagation of the sinusoidal part of the signal. This evaluation will be done 


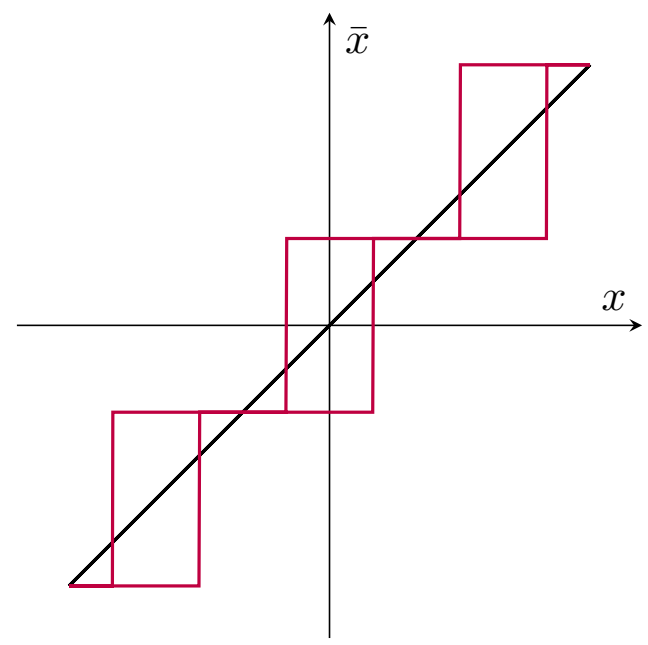

Figure 5.8: Input-Output relationship of the sampler used in Example 18 (Parameters: $\Delta \epsilon / \epsilon=\Delta \delta / \delta=0, \epsilon / \delta=1$ and $h / \delta=1 / 2)$.

with the expression presented in equation (5.1), which also depends on the bias $B$. If the pairs $\{A, B\}$ that fulfill the corresponding expression in Table B.1 are also those that intersect the open-loop transfer function in the Nyquist diagram, analytically:

$$
G_{o l}\left(j \omega_{o}\right)=-\frac{1}{\mathcal{N}_{A}(A, B)},
$$

then a limit cycle oscillation of the type $A \sin \left(\omega_{o} t\right)+B$ will be obtained.

This concept will be introduced through the following example:

Example 18. Consider a process with transfer function

$$
G(s)=\frac{1}{(s+1)^{3}},
$$

and for illustrative purposes, consider that this process is placed in a loop with a sampler with $\Delta \epsilon / \epsilon=0, \Delta \delta / \delta=0, \epsilon / \delta=1$ and $h / \delta=1 / 2$, whose input-output relationship is shown in Figure 5.8, without any controller.

This case will correspond to the stability condition for the bias where both controller and process have neither poles nor zeros at the origin presented in Table B.1. Adapting that equation to the current case, $L_{1}(0)=1$ and $L_{2}(0)=G(0)$, resulting in:

$$
B\left(1+\mathcal{N}_{B} G(0)\right)=r(\infty)-p(\infty) G(0)
$$

Five scenarios will be considered:

1. Unitary reference input without disturbance. 


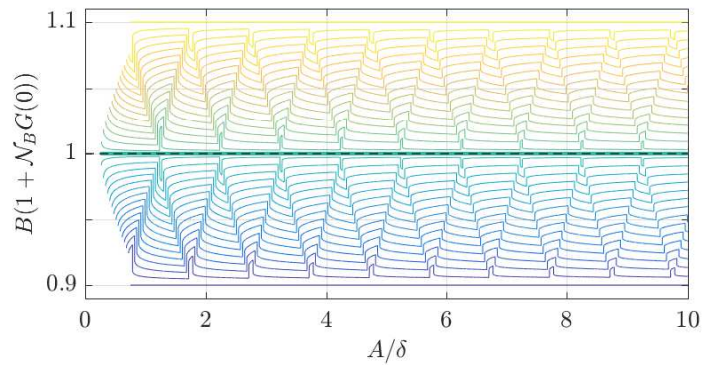

(a) Bias.

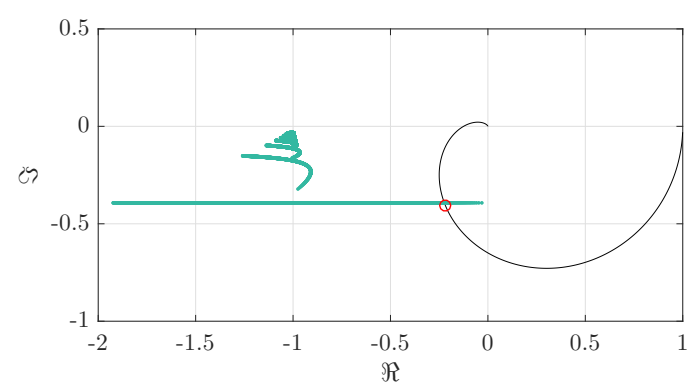

(b) Dynamic.

Figure 5.9: Graphical representation of the oscillation conditions for Scenario 1.

2. Reference input of magnitude 0.95 without disturbance.

3. Unitary reference input and disturbance of magnitude 0.95 .

4. Same as scenario 3 but increasing the gain of the process.

5. Same as scenario 3 but doubling the value $\epsilon$ of the sampler.

Scenario 1: In this case, the equation for the maintenance of the bias is simplified, resulting in:

$$
B\left(1+\mathcal{N}_{B} G(0)\right)=r(\infty)
$$

The central value $B_{c}$ can be obtained from equation (5.6) as:

$$
B_{c}=\frac{r(\infty)}{1+\epsilon / \delta G(0)}=\frac{1}{2}
$$

Thus, the set of values to evaluate will be $B_{s} \in\left[B_{c}-\delta / 2, B_{c}+\delta / 2\right]$. A width of the set equal to $\delta$ is chosen because of the symmetry properties of $\mathcal{N}_{A}$ explained in the previous section. The set of values $B_{s}$ has been evaluated with equation (5.8). Regarding that expression, the left hand term varies with each term of the set $B_{s}$ evaluated, while the right hand term is constant. Therefore, the solutions $A$ and $B$ to that expression can be easily found by representing the left hand term, and the horizontal line representing the right hand term and evaluating the intersections between them.

The graphical representation of the left hand term has been presented in Figure 5.9a, each trace representing an item of the set. As it can be seen, it only exists one trace that equals to $r(\infty)$, the right hand term, which has been represented by a dashed black line. The trace that exactly matches the dashed black line corresponds to the trace obtained for $B=0.5$. As all the values of that trace are equal to the searched solution, if the inverse negative of $\mathcal{N}_{A}$ intersects at any point with the open-loop transfer function, then an oscillation will appear. The oscillation condition for the dynamic part has been presented in Figure 5.96 where it can be seen that $-1 / \mathcal{N}(A, B=0.5)$ intersects with $G(j \omega)$. 


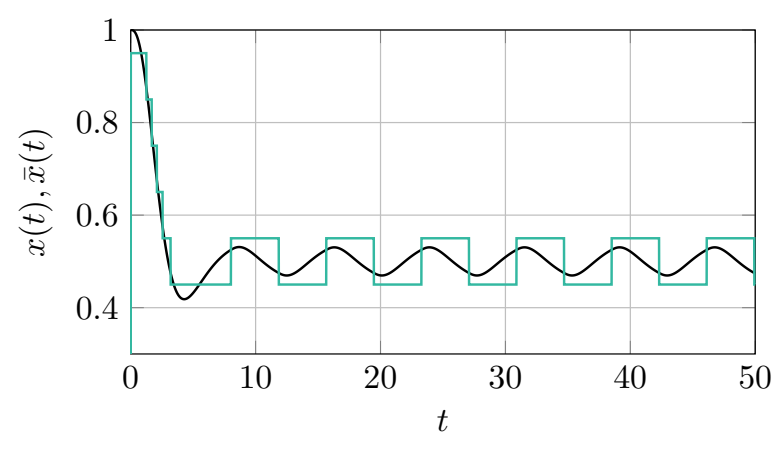

Figure 5.10: Temporal response of the error $x(t)$ and sampled error $\bar{x}(t)$ signals under the conditions of the Scenario 1.

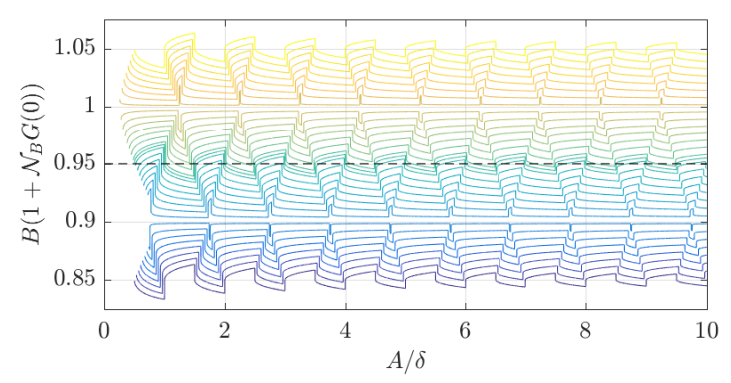

(a) Bias.

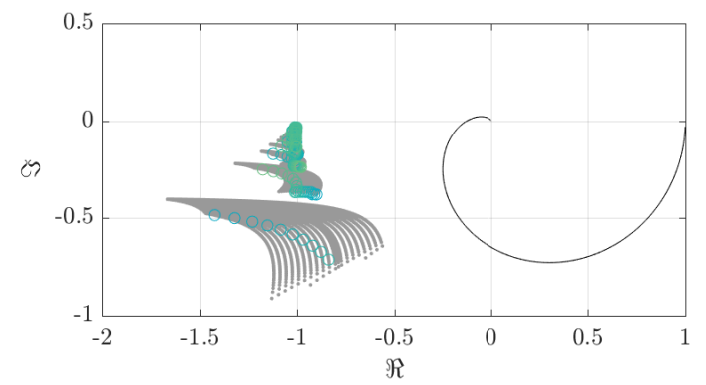

(b) Dynamic.

Figure 5.11: Graphical representation of the oscillation conditions for Scenario 2.

The temporal response of the process under the conditions presented in this scenario has been obtained, and the error and sampled error signals have been presented in Figure 5.10. The oscillation period has been measured in this figure and $G\left(j \omega_{o}\right)$ has been represented in the Nyquist diagram in Figure $5.9 b$ with a red circle, proving the validity of the method.

Scenario 2: To evaluate the maintenance of the bias, equation (5.8) remains valid because only the magnitude of the signal has changed. The procedure to obtain the set of values of bias $B_{s}$ is also the same, firstly the central value is obtained, in this case $B_{c}=0.475$, and then a margin of $\pm \delta / 2$ for symmetry reasons is established.

The set of values $B_{s}$ has been evaluated with equation (5.8) and the graphical representation of the left hand term has been presented in Figure 5.11a, each trace representing an item of the set. A dashed black line has been added representing the right hand term of equation (5.8). The intersections between right and left hand terms constitute a set of pairs $\{A, B\}$ that will allow the maintenance of the bias in the oscillation. However, it must be evaluated if the dynamic part is also maintained through the loop. Hence, in Figure $5.11 b$ the inverse negative of $\mathcal{N}_{A}$ for each value of $B$ in the pair have been presented in gray, the matching values of $A$ have not been considered and instead a wide range of $A$ has been swept for each $B$. This representation in gray matches with the classical DF plot, where all the parameters are fixed and only the amplitude of the oscillations varies. Then, the specific points $-1 / \mathcal{N}_{A}(A, B)$, where the pairs 


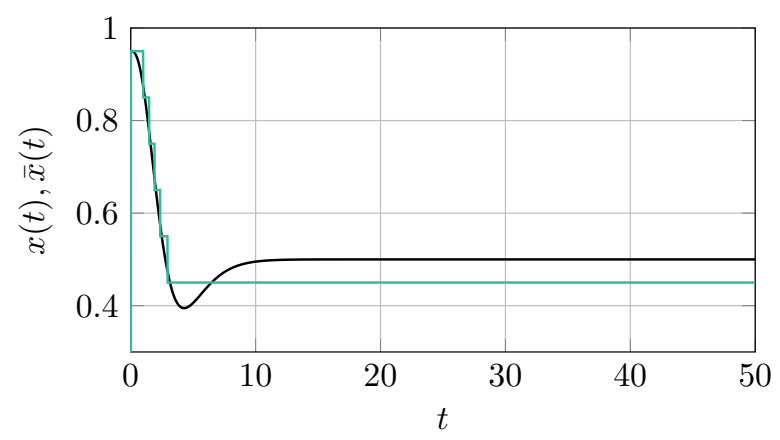

Figure 5.12: Temporal response of the error $x(t)$ and sampled error $\bar{x}(t)$ signals under the conditions of the Scenario 2.

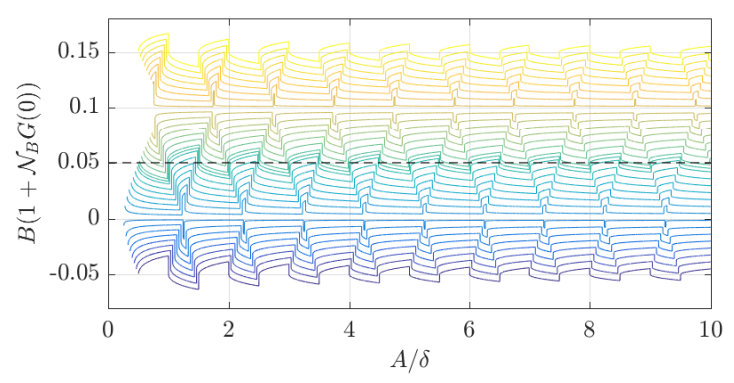

(a) Bias.

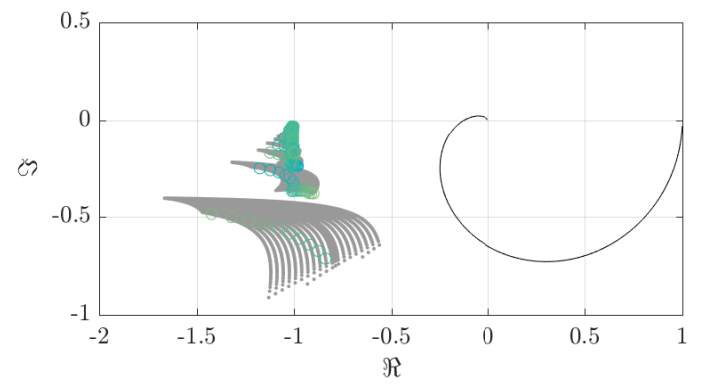

(b) Dynamic.

Figure 5.13: Graphical representation of the oscillation conditions for Scenario 3.

$\{A, B\}$ are solution of equation (5.8), have been encircled. The plots in gray are illustrative and not necessary for the stability analysis. As it can be seen, the open-loop transfer function does not intersect with any of the highlighted points, therefore, no oscillations will take place, and so it has been observed in the simulation presented in Figure 5.12.

Scenario 3: In this scenario, a disturbance signal is present on the loop, therefore, the expression to determine the central value $B_{c}$ obtained from equation (5.6) is:

$$
B_{c}=\frac{r(\infty)-p(\infty) G(0)}{1+\epsilon / \delta G(0)}=\frac{1-0.95}{2}=0.025 .
$$

A set of values for the bias as in the previous cases have been evaluated, obtaining the representation of the left hand term of equation (5.7) presented in Figure 5.13a. In this figure, the dashed black line presented corresponds to the right hand term of equation (5.7). A set of pairs $\{A, B\}$ is obtained, and as in the previous case, it can be observed in Figure $5.13 b$ that no intersection between the inverse negative of the DIDF evaluated at those points and the open-loop transfer function is observed. Hence, no oscillation will take place.

This result evinces that, in this type of loop architectures, the disturbance input can act both as a stabilizing or destabilizing agent. In Figure 5.14, the previous statement can be seen. The first half of the experiment corresponds to the temporal response of the error signal to the reference 


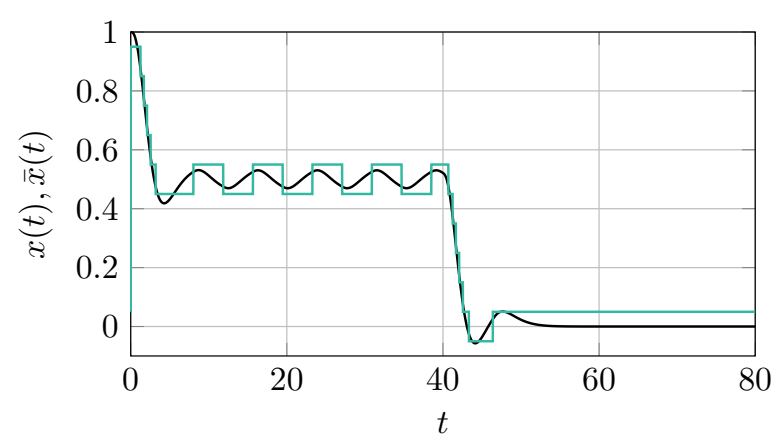

Figure 5.14: Temporal response of the error $x(t)$ and sampled error $\bar{x}(t)$ signals under the conditions of the Scenario 3.

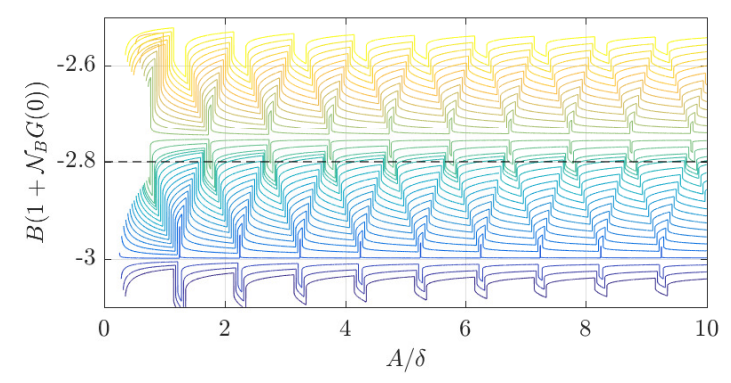

(a) Bias.

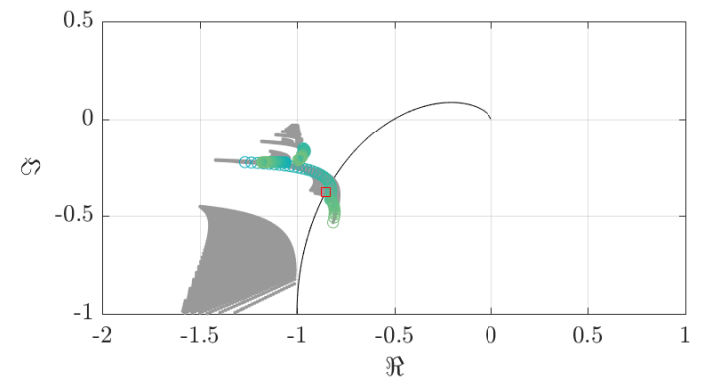

(b) Dynamic.

Figure 5.15: Graphical representation of the oscillation conditions for Scenario 4.

input change, which also corresponds to Scenario 1, where it was proven the oscillatory behavior. However, by applying the disturbance change at $t=40 \mathrm{~s}$ the process manages to stabilize within the evaluated range of biases.

Scenario 4: In this scenario, there will be reference and disturbance changes and the process gain will be 4 times the original. For the sake of brevity the repetitive steps followed above are omitted.

The graphical representation of the oscillation conditions is presented in Figure 5.15. In Figure 5.15a, the graphical representation of the left hand term of equation (5.7) is presented with different colored lines and the right hand term of that equation is presented with a dashed black line. The intersections between both terms represent the solutions of the equations from which the pairs $\{A, B\}$ are obtained. Then, in Figure 5.15b, all the traces $-1 / \mathcal{N}_{A}$ are presented for the different values of $B$ and the specific solutions are highlighted. In this last figure, it can be observed that there exist an intersection between $G(j \omega)$ and some of the highlighted points, revealing that a limit cycle oscillation can occur.

The temporal response of this system has been obtained and it is represented in Figure 5.16. In this simulation, the reference change has been applied at $t=0 \mathrm{~s}$ and the disturbance at $t=40 \mathrm{~s}$. In this scenario, it can be seen that two different limit cycle oscillations are obtained, the first one with $m_{\text {sup }}+m_{\text {inf }}=1$ resulting from the application of a step change, which has 


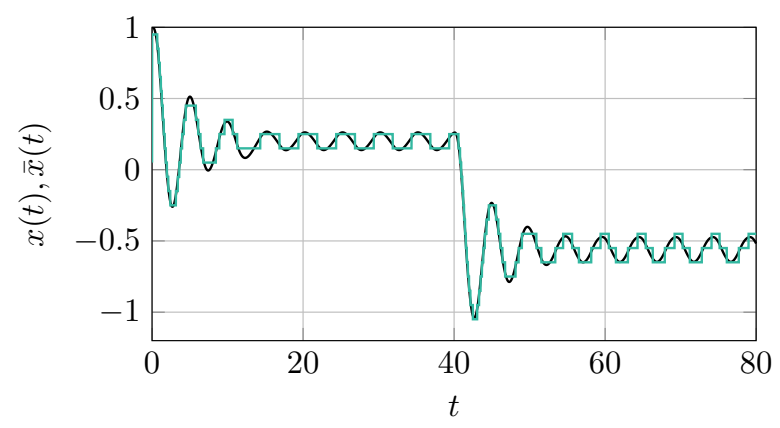

Figure 5.16: Temporal response of the error $x(t)$ and sampled error $\bar{x}(t)$ signals under the conditions of the Scenario 4.

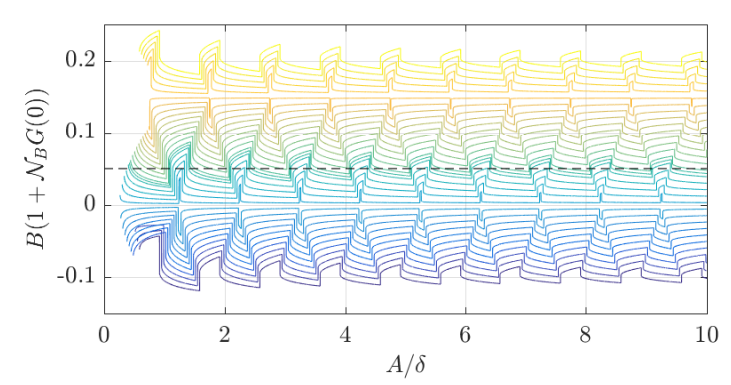

(a) Bias.

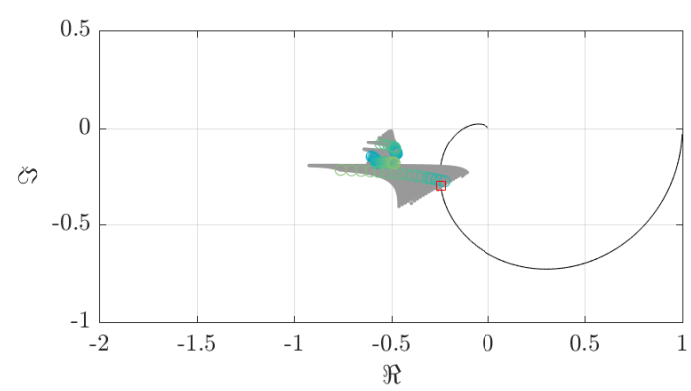

(b) Dynamic.

Figure 5.17: Graphical representation of the oscillation conditions for Scenario 5.

not been evaluated theoretically, but it could be done as in the Scenario 1. The second limit cycle is the expected result of the study in this scenario, with $m_{\text {sup }}=m_{\text {inf }}=1$ after the application of the disturbance change. The period of the resulting oscillation has been measured and $G\left(j \omega_{0}\right)$ has been represented in the Nyquist diagram in Figure $5.15 b$ with a red square, validating the prediction of the DIDF method.

The apparition of different type of oscillations modifying the disturbance or reference input signals can be interesting for the identification of processes, in which different types of oscillations with different oscillation periods can be used to identify multiple points of the frequency response of a process. However, for those cases the correct case study regarding the equations must be applied.

Scenario 5: In this final scenario, the ratio $\epsilon / \delta$, that so far in precedent scenarios was 1, has been doubled. Thus, this change must be introduced in the calculation of the central value:

$$
B_{c}=\frac{r(\infty)-p(\infty) G(0)}{1+\epsilon / \delta G(0)}=\frac{1-0.95}{3}=0.0167 .
$$

As in previous scenarios, a set of values $B_{s}$ is obtained to compute the stability conditions, obtaining the graphical representations presented in Figure 5.1\%. From these figures it can be seen that the DIDF method predicts an oscillation. 


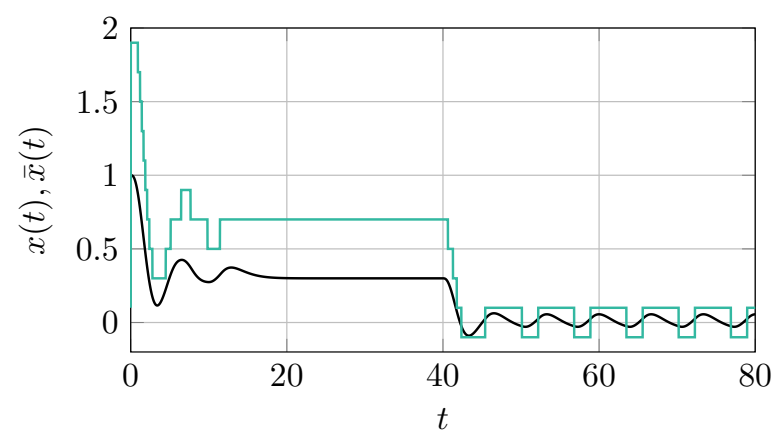

Figure 5.18: Temporal response of the error $x(t)$ and sampled error $\bar{x}(t)$ signals under the conditions of the Scenario 5.

The system has been tested in simulation obtaining the temporal response presented in Figure 5.18. The period of the obtained oscillation has been measured and $G\left(j \omega_{o}\right)$ has been represented in the Nyquist diagram of Figure 5.17b with a red square proving the validity of the prediction.

In this scenario, the opposite case to the presented in the Scenario 3 can be seen, starting from a stable temporal response as a consequence of the reference change, the application of the disturbance input to the system acts as a destabilizing agent, resulting in the apparition of a limit cycle oscillation.

\subsection{Conclusion}

In this chapter, the tools to characterize the limit cycle oscillations induced by any kind of multilevel Fixed Threshold Sampler (FTS) have been provided. To that end, the structure of these kinds of samplers have been parametrized and it has been shown that by choosing some specific set of parameters some of the most used quantifiers can be obtained. Some notable specific cases are RQ quantization, Symmetric-Send-On-Delta or any kind of multi-level relays.

In order to characterize the oscillations induced by these samplers the Describing Function technique has been used. Since depending on the set of parameters chosen for the sampler oscillations with a bias can be obtained, a variant called the Dual Input Describing Function (DIDF) has been used, which already contemplates the apparition of this bias.

The DIDF has been obtained for the generalized parameters describing any FTS. In addition, the DIDF involving the dynamic part has been deeply analyzed, revealing some interesting effects of the parameters on its traces and some properties such as periodicity and symmetry depending on the bias.

The guidelines to perform the robustness analysis have been provided. In the analysis study, it has been shown the paramount importance of the loop configuration and the signals involved in the loop, because they have a direct effect on the limit cycle oscillation conditions. Some examples with specific cases have been included to show how the analysis methodology should be applied. From these examples some features like the appearance of different types of oscillations 
produced by the application of some signals have been revealed, which can be used in further works for identification purposes. 



\title{
Sampled SSOD analysis with DF method
}

\begin{abstract}
In this chapter, the robustness of discrete PI controllers when used with a Symmetric-Send-On-Delta (SSOD) sampling law is addressed. Hitherto, the continuous Describing Function has been employed as a suitable tool to evaluate the robustness of such systems against limit cycles oscillations induced by the SSOD. However, due to the discrete implementation of the controllers in most of the actual applications, the Sampled Describing Function technique is used in this chapter to provide a more realistic approach, which takes into account the effect of both the SSOD and sampling period of the discrete controller on inducing such oscillation. A simple measure has been developed to characterize the robustness of these systems and has been tested through several examples, showing its validity in predicting the apparition or avoidance of limit cycles. This measure has been used to evaluate the robustness of some spread tuning rules applied to a wide batch of systems reflecting the dynamics of most processes in the industry.
\end{abstract}

\subsection{Introduction}

Event-Based Control (EBC) constitutes a solid alternative to classical time driven control on distributed control systems because it reduces the data drop out, decreasing the delays and minimizing the packet losses in the communication networks. These controllers attain this objective as a consequence of their data send policy, which only sends new data when significant changes on the state of the system are produced, instead of periodically as classical time driven controllers do.

Therefore, this data send policy becomes crucial in EBC, because it is in charge of generating the events for the execution of the controller's algorithm. Among the different event generation 
techniques, the ones based on the signal quantification have become more important because of their ease of implementation. That is the case of the well know Send-On-Delta (SOD) sampling strategy, which sends data whenever the signal changes more than a certain value $\delta$ from the last sample. This sampling technique has been used in several works proving its effectiveness in terms of control performance and communication reduction [19, 50].

Several variations on the SOD sampling strategy have been presented, most of them considering the thresholds fixed and no longer depending on the last value taken. Among these variations the Regular Quantization was studied in terms of robustness in [58], and a variation of this last sampling strategy was presented in [42], in which a customizable hysteresis was added to the sampling to avoid bursts of events due to noise. However, one of the most known variations of the SOD sampling was presented in [10], and it is known as Symmetric-Send-OnDelta (SSOD). This strategy presents fixed thresholds of value $\delta$ and introduces a hysteresis of the same value $\delta$, being its input-output relationship symmetric.

One of the main points in the analysis and design of event-based control systems is the existence and avoidance of limit cycle oscillations in the closed-loop response. The characterization of steady-state oscillations for different kind of systems under a SSOD sampling strategy has been presented in [17]. This analysis has been treated in other works using the Describing Function (DF) technique $[60,56]$, in which, in addition, tuning methods for PI controllers within a control structure with a SSOD sampler have been obtained. The use of the DF allows extending some concepts of the classical control theory, like the gain and phase margins, to the analysis and design of EBC systems. However, other analysis techniques can be used such as the Tsypkin method, which has been specifically used to analyze the robustness of SSOD-PID structures in [44].

In all the theoretical studies about SSOD based control systems a continuous approach has been adopted towards the controller implementation, i.e. the PID is considered to be continuous and therefore all the results have been obtained under this assumption. In networked control systems, however, the controllers are always implemented in micro-processor based devices considering a discrete approximation. The aim of this chapter is to study the effect of the sampling time in the robustness against limit cycle oscillations when a discrete implementation of the PI controller is used jointly with the SSOD sampling strategy, as is the cases of the practical applications of the SSOD-PI presented in $[12,54,8,52,53]$. In neither of those papers the influence of this parameter has been addressed.

Tuning methods for continuous PID are applied by the control practitioners in many industrial settings without bearing in mind that discrete versions of the controllers are executed in microprocessor-based systems. In most of these cases the digital implementation of the controller does not have a detrimental effect in the loop because the sampling frequency used by the control algorithm is high enough to consider the controller as a continuous one. In this sense, it should be taken into account that the sampling time required for some common process variables such as flow, level, pressure or temperature is in the order of seconds meanwhile the commercial digital controllers have sampling intervals in the order of milliseconds. 
Despite the aforementioned fact, it is well known that the sampling time plays an important role in discrete time control systems. High values of sampling time could degrade the control performance or even produce instability. On the other hand, the minimum value of the sampling interval is limited by hardware and software restrictions of the microprocessor-based system where the control algorithms are executed. Because of the relevance of the sampling time, several criteria have been developed for selecting this parameter taking into account both the frequency or time response of the control loop, [31]. A general rule of thumb for the PI controllers states the sample time in the range of 0.1 to 0.3 of integral time in order to obtain good performance and acceptable robustness of the control systems [6].

This chapter addresses the robustness of SSOD based control systems when a discrete PI is used. This consideration matches with the reality of computer based implementation of controllers which are almost executed as a periodic algorithm. The analysis is based on the Sampled Describing Function [37] which takes into account the SSOD quantification and the execution period of the controller. The main characteristics of this DF have been studied, revealing the influence of its parameters in the behavior of this kind of control systems. Some guidelines are given to evaluate the existence of limit cycle using a new robustness measure proposed in the chapter. Because of the lack of specific tuning rules for the scheme under study, the robustness of controllers provided by several classical and spread tuning rules for continuous PI have been evaluated, namely, Ziegler-Nichols [79], AMIGO [4], One-Third [27] and SIMC [68] tuning rules. These methods have been applied to a batch of models that gather the most common dynamics in industrial processes, and their robustness in the studied loop structure has been evaluated using the proposed measure.

The chapter is organized as follows. In section 6.2 the loop structure and the general problematic are presented. In section 6.3 the Sampled Describing Function that characterizes the non-linear behavior of the system is presented and studied. In section 6.4 guidelines about how to perform the stability analysis are given, offering a systematic approach to evaluate the robustness and proposing a specific robustness measure. In section 6.5 several tuning methods are used to tune controllers for a given batch of models and their robustness in the proposed loop structure is evaluated. Finally, in section 6.6 the conclusions about the chapter are drawn.

\subsection{Problem statement}

Consider the networked control system presented previously in Chapter 4 in Figure 4.1, where $C(s)$ and $G(s)$ are the controller and the process transfer functions respectively, $y_{r}$ is the reference signal to be tracked, $y$ is the controlled output, and $p$ is the disturbance input. The controller is assumed to be located near the actuator and the sensor sends samples of the process output represented by $y$ (it could also be of the tracking error $e$ ) to the controller through the communication network whose communication delays are modeled by the term $\exp \left(-t_{d} s\right)$. The sensor unit employs a SSOD strategy for sampling the input signal: a new value $e^{*}=i \delta, i \in \mathbb{Z}$ is sent to the $\mathrm{ZOH}$ when $e$ crosses the $i \delta$ levels and $\bar{e}$ maintains its value for $\pm \delta$ variations around 


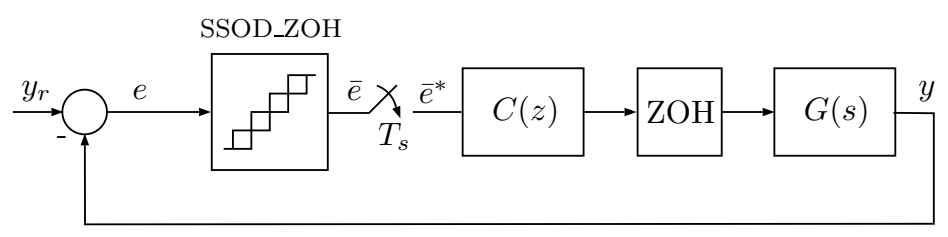

Figure 6.1: Sampled system configuration with SSOD non-linearity.

the $i \delta$ levels. This behavior is described by Equation (6.1). It is worth remarking that other sampling strategies such as RQH presented in [42] or asymmetric multi-level relays could be used, but SSOD is simpler to implement, has more literature associated addressing the tuning of controllers under this sampling and has been proved in several practical environments.

$$
\bar{e}(t)= \begin{cases}(i+1) \delta & \text { if } e(t) \geq(i+1) \delta \text { and } \bar{e}\left(t^{-}\right)=i \delta, i \in \mathbb{Z} \\ (i-1) \delta & \text { if } e(t) \leq(i-1) \delta \text { and } \bar{e}\left(t^{-}\right)=i \delta \\ i \delta & \text { if } e(t) \in[(i-1) \delta,(i+1) \delta] \text { and } \bar{e}\left(t^{-}\right)=i \delta\end{cases}
$$

This schema, and the control problem associated to it, was first proposed in [10] and it has been treated in different ways in the literature. In [11] a tuning method for this kind of structure based on other tuning rules such as AMIGO [28] and SIMC [68] tuning rules was presented. In $[60,56]$ the authors rely on the DF approach to characterize the robustness against limit cycles produced by the SSOD non-linearity, and propose a tuning method that takes this fact into account. In [44] the Tsypkin method has been used to better characterize the robustness to limit cycle of this kind of systems without the restrictions imposed by the DF about the filtering properties of $G(s)$.

The works mentioned above follow the approach of considering a continuous controller in the loop. However, this approach can induce to unexpected errors when the controllers are implemented in computer-like devices such as PLCs or other electronic cards. This fact modifies the approach to the problem presented in Figure 4.1, because neither the signal that arrives to the controller nor the controller are continuous; instead the signal is periodically sampled by the controller module to recalculate the control action, which is kept constant during the sampling time.

With the considerations already described above, the system in Figure 4.1 admits the Hammerstein-Wiener representation presented in Figure 6.1, being the block SSOD_ZOH the combination of the SSOD and ZOH blocks, and the implementation of the discrete PI being modeled by equation (6.2), which has been obtained by applying the bilinear transform. This new block diagram describes the actual problem behind the networked control systems under study more accurately.

$$
C(z)=K_{p}+\frac{K_{p}}{T_{i}} \frac{T_{s}}{2} \frac{z+1}{z-1}
$$

One of the main issues when dealing with this kind of systems is the apparition of limit cycle oscillations, which are induced by the SSOD non-linear behavior and sampling. In Figure $6.2 \mathrm{a}$ 


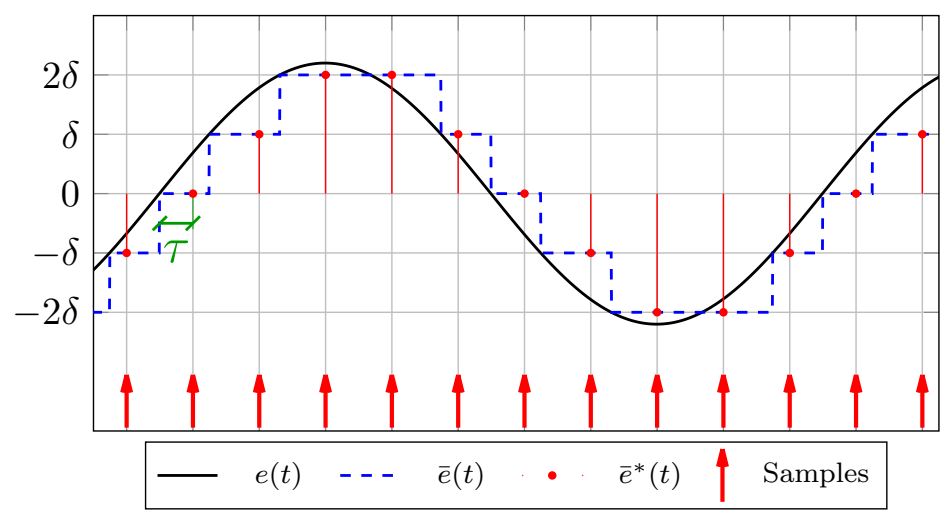

Figure 6.2: Sine wave in black, quantified by the SSOD_ZOH block in dashed blue, and sampled according to a given sampling period in red (sampling time indicated with red arrows).

typical limit cycle oscillation is shown. As it can be seen, the error signal $e$ is not only quantified by the SSOD non-linearity, which results in $\bar{e}$, but it is also sampled afterwards, obtaining the samples $\bar{e}^{*}$, which constitute the input to the controller to compute and actualize the control action.

This quantification and posterior sampling constitute the source of limit cycle oscillations apparition. Therefore, to evaluate the robustness of a given system, an analysis methodology which takes into account the peculiarities of this kind of sampled system is presented.

\subsection{Sampled Describing Function approach}

To study non-linear systems with sampling elements, as that shown in Figure 6.1, there exist several methods, some of them exact as the one presented in [40], but very complicated for practical uses. Other methods like the ones based on the Describing Function offer an approximate estimation of the robustness, which is precise when a filtering linear part is present, while being easier to develop. Two variations on the original Describing Function technique can be used in this case, namely, the z-transform Describing Function [36] and the Sampled Describing Function [37], which will be used in this chapter due to its simplicity, [25].

The condition to avoid limit cycle oscillations is defined by:

$$
G_{o l}(j \omega) \neq-\frac{1}{\mathcal{N}} ; \quad \forall \omega
$$

where $G_{o l}(j \omega)$ represents the open-loop transfer function of all the linear elements, i.e. network delay, controller, $\mathrm{ZOH}$ and system. $\mathcal{N}$ is the describing function of the non-linear part, which in this case characterizes the SSOD_ZOH block and the sampler. It can be proven (see Appendix 
B.4) that the Describing Function $\mathcal{N}$ is given by the following equation:

$$
\begin{aligned}
& \mathcal{N}\left(\delta / A, r, \tau, T_{s}\right)=\frac{2 \delta}{\pi T_{s} A} \sum_{k=-\infty}^{\infty} \frac{e^{-j k 2 \pi \tau / T_{s}}}{r k-1}\left[\sum _ { i = 1 } ^ { m - 1 } \left\{\left(-\sqrt{1-\left(\frac{i \delta}{A}\right)^{2}}+j \frac{i \delta}{A}\right)^{r k-1}\right.\right. \\
& \left.\left.-\left(\sqrt{1-\left(\frac{i \delta}{A}\right)^{2}}+j \frac{i \delta}{A}\right)^{r k-1}\right\}-\left(\sqrt{1-\left(\frac{m \delta}{A}\right)^{2}}+j \frac{m \delta}{A}\right)^{r k-1}+(-1)^{r k-1}\right]
\end{aligned}
$$

where $m=\left\lfloor\frac{A}{\delta}\right\rfloor$ is the number of levels crossed, $\tau$ is the lag between the zero-crossing of the signal and the first sample taken (see Figure 6.2) and $r$ is the ratio between the oscillation period $T_{o}$ and the sampling period $T_{s}$.

From expression (6.4) it can be observed that, as in the case of the continuous DF of the SSOD quantization [56], the shape of the sampled DF does not depend on the specific value of $\delta$, but on its relationship with the amplitude $A$ of the induced oscillation, which is expressed by the quotient $\delta / A$. Therefore, the robustness does not depend on the quantization level since varying $\delta$ will also modify the amplitude of the oscillation proportionally, resulting in the same ratio $A / \delta$ and, consequently, in the same points on the Nyquist diagram.

According to [25], $r$ must be an integer value, otherwise the oscillation may contain harmonic components with frequencies lower than the fundamental frequency, which cannot be discarded with the filtering hypothesis. In addition, in those cases where $r$ is considered to be odd, the samples taken in each semi-period of the oscillation are different, this leads to an asymmetry which can be relevant for those cases where a low number of samples per period are taken; in the other cases, this difference is irrelevant.

Figure 6.3 depicts the shapes of $-1 / \mathcal{N}$ for different values of $r$. The locus of $-1 / \mathcal{N}$ is composed of several branches, one for each value of $m$, each of them has been represented with a different color. Bigger values of $m$ tend to approximate the traces of the DF to the point $\left(-T_{s}, 0\right)$ and as the value of $m$ is reduced the branches expand towards the third quadrant. The clearer case is the one presented in the Figure $6.3 \mathrm{~d}$ where each branch is visibly well defined and it reassembles the DF of the SSOD (without sampling) [56]. This happens because for that Sampled-DF the ratio $r$ is big enough to consider the effect of the sampling negligible. Nevertheless, for the other cases it can be seen how decreasing that ratio tends to widen the size of the branches, deforming them and making them unintelligible from one another.

In [25] the concept of oscillation mode is presented for a relay non-linearity. In that case, a $n, n$ mode is defined as a cycle in which $n$ positive drive pulses are followed by $n$ negative drive pulses. This concept can be applicable to the oscillations produced by the non-linear structure studied in this chapter introducing a slight difference due to the non-linearity characteristics.

Consider a one-leveled oscillation $(m=1)$, sampled in such a way that $r=T_{o} / T_{s}=6$. With this sampling rate, six samples are taken in an oscillation period. In a semi-period, the values 


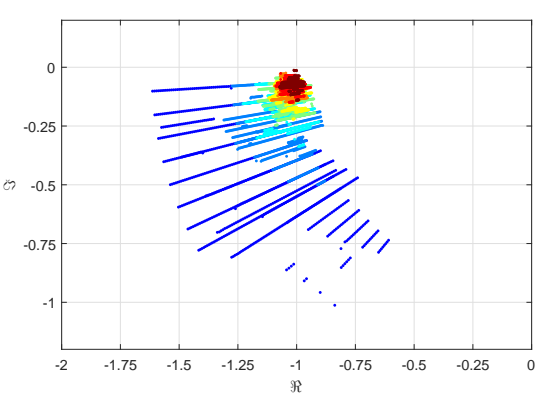

(a) $r=10$.

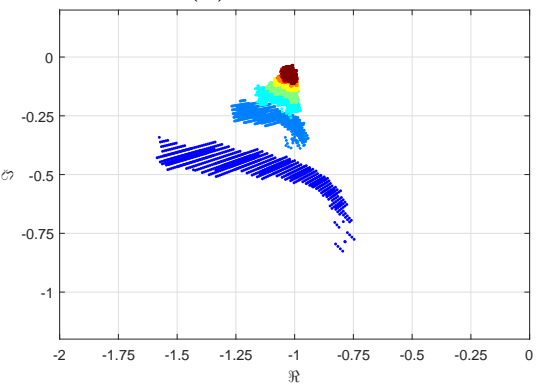

(c) $r=50$.

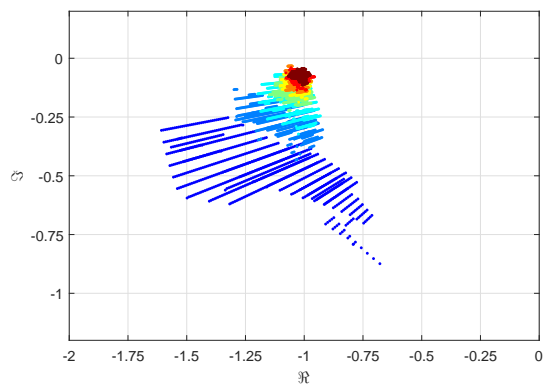

(b) $r=20$.

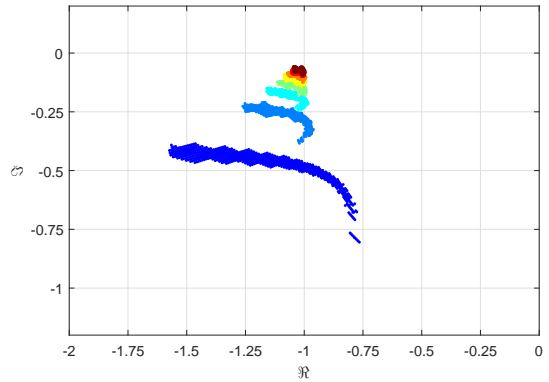

(d) $r=100$.

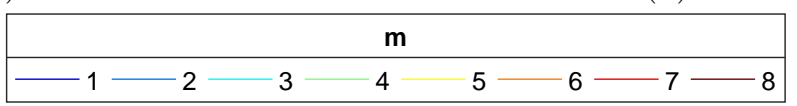

Figure 6.3: Sampled DF for different values of $r$, all of them considering $T_{s}=1$.

that these samples can have are either $\bar{e}^{*}\left(k T_{s}\right)=0,0, \delta$ or $\bar{e}^{*}\left(k T_{s}\right)=0, \delta, \delta$ for $k=1,2,3$. Both examples are shown in Figures $6.4 \mathrm{a}$ and $6.4 \mathrm{~b}$ respectively. Note that on the other semi-period the samples for $k=4,5,6$ would be the same but with opposite sign. These two combinations are the only two possible modes for the considered sampling rate maintaining the amplitude of the oscillation.

Focusing on the simpler mode type, which are those where $m=1$, the number of modes for a given value of $r$ can be characterized as follows. As it has been shown, the modes in SSOD sampled oscillations are characterized by samples in a lower level $\bar{e}^{*}=0$ and samples in a high level $\bar{e}^{*}=\delta$ and its symmetric in the second semiperiod. In addition, as the amplitude

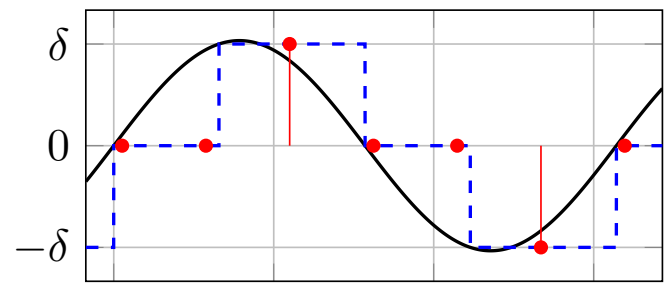

(a) $\bar{e}^{*}\left(k T_{s}\right)=0,0, \delta$.

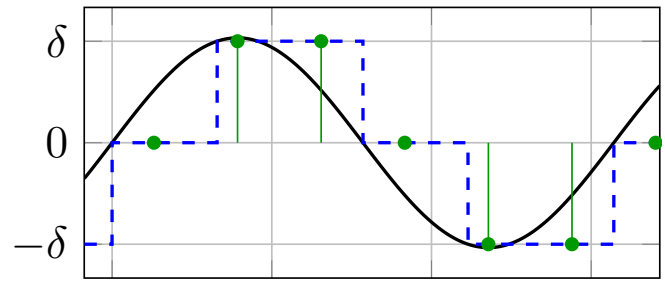

(b) $\bar{e}^{*}\left(k T_{s}\right)=0, \delta, \delta$.

Figure 6.4: Sine wave quantified by a SSOD and sampled resulting in two different oscillation modes. 


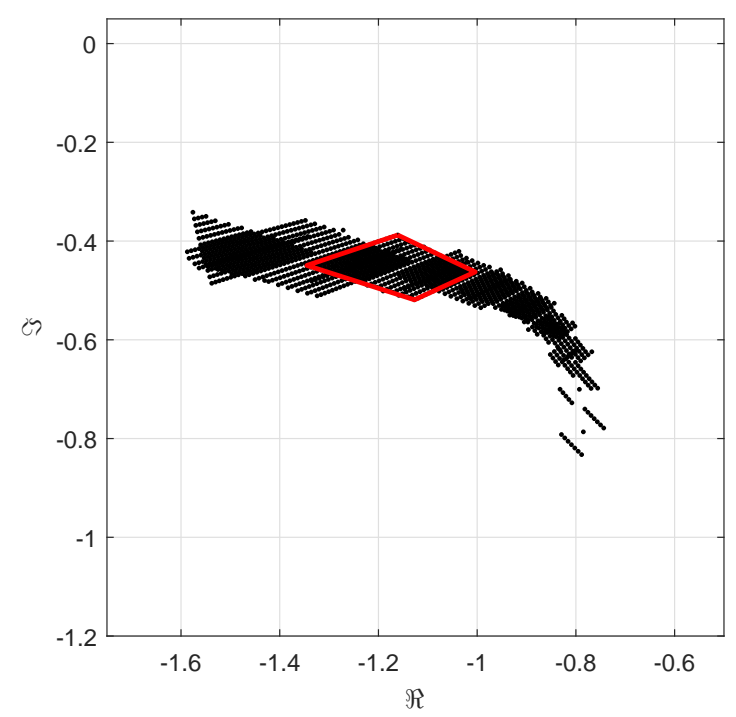

Figure 6.5: Detail of the branch $m=1$ of the Sampled DF with $T_{s}=1$ and $r=50$ with a mode region highlighted.

of the oscillation increases the temporal frame before $\bar{e}$ commutates to a high level is reduced. Therefore, the number of modes can be obtained as the difference between the maximum and the minimum number of samples that fit in the temporal frame before commutation. Then, considering the two extreme cases for the amplitude in the case $m=1$, which are $A=\delta$ and $A=2 \delta$, and as $e$ is a sinusoid, the time in which the switch is produced $(e=\delta)$ can be obtained, which is $t_{1}=T_{o} / 4$ and $t_{2}=T_{o} / 12$. The number of samples that fit in that temporal frame is obtained dividing by $T_{s}$, and then, just counting the difference between the maximum and minimum number of samples that fit in that temporal frame, the number of modes for a given $r$ in $m=1$ is obtained as:

$$
n^{\circ} \text { modes }=\left\lceil\frac{r}{4}\right\rceil-\left\lfloor\frac{r}{12}\right\rfloor+1 .
$$

From this expression it can be observed that the number of possible modes increases with the sampling ratio $r$, intuitively, decreasing the sampling period increases the number of samples in that temporal frame, being the difference between the extreme cases greater.

The influence of the ratio $r$ on the oscillation modes is reflected in the DF traces. In Figure 6.5 it has been represented the branch that corresponds to $m=1$ of the Sampled DF with $r=50$. It can be seen some overlapping rhomboid regions (one of them surrounded in red), which are crossed by several straight lines. Each of those rhomboid regions corresponds to a different oscillatory mode, i.e. the oscillations obtained when intersecting those regions have a certain number of samples in each level.

Within the rhomboid regions some straight lines appear. Each of these series of traces corresponds to a different initial lag $\tau$ when evaluating the DF swapping the ratio $\delta / A$. This implies that multiple combinations of ratios $\delta / A$ and initial lags $\tau$ can generate the same limit 
cycle oscillation. This can be easily seen from the oscillation mode shown in Figure 6.4, where the represented modes can be obtained for different combinations of the sine amplitude $A$ and initial sampling delay $\tau$.

The oscillation modes depend on the sampling rate, but also on the ratio $\delta / A$, which propitiates the existence of modes in which several levels are crossed $(m>1)$. This kind of modes involves an additional complexity for its analysis due to the apparition of a curious phenomena: if the sampling rate is not sufficient, a level may not have any sample on it, resulting in more overlapping regions. As the main goal of this contribution is to avoid any type of oscillation modes, we will not focus on their study, however, it is important to notice that the dispersion of the DF traces with $r$ can be explained with the understanding of the concept of modes. With low ratios of $r$ fewer number of modes are possible and more combinations of parameters are possible to obtain them, generating a great dispersion of the DF traces. On the other hand, with higher ratios of $r$ more modes (rhomboid regions) appear, but they are smaller because the parameters admit less variation to maintain the mode.

\subsection{Stability analysis}

One of the main considerations when studying the networked control systems presented in this chapter is the avoidance of limit cycle oscillations induced by SSOD sampling law. These oscillations can be prevented if the condition described by equation (6.3) is fulfilled, which relates the non-linear part, characterized by the $\mathrm{DF} \mathcal{N}$, and the linear part of the system.

Considering firstly the linear part of the system in Figure 6.1, the open-loop transfer function $G_{o l}(s)$ that includes the discrete controller $C(z)$, the $\mathrm{ZOH}$, the time delay introduced by the network and the system $G(s)$ must be obtained. The transfer function of the $\mathrm{ZOH}$ is:

$$
Z O H(s)=\frac{1-e^{-s T_{s}}}{s}
$$

Then, the transformation $z=e^{s T_{s}}$ is applied to the transfer function of the discrete controller, given by equation (6.2), to obtain the starred transform of the controller $C^{*}(s)$, obtaining:

$$
G_{o l}(s)=C^{*}(s) Z O H(s) G(s)
$$

To facilitate the stability analysis, condition (6.3) has been rewritten as:

$$
\frac{G_{o l}(s)}{T_{s}} \neq \frac{-1}{\mathcal{N}^{\prime}} ; \quad \forall \omega
$$


where:

$$
\begin{gathered}
\mathcal{N}^{\prime}\left(\delta / A, r, \theta_{s}\right)=\frac{2 \delta}{\pi A} \sum_{k=-\infty}^{\infty} \frac{e^{-j k \theta_{s}}}{r k-1}\left[\sum _ { i = 1 } ^ { m - 1 } \left\{\left(-\sqrt{1-\left(\frac{i \delta}{A}\right)^{2}}+j \frac{i \delta}{A}\right)^{r k-1}\right.\right. \\
\left.\left.-\left(\sqrt{1-\left(\frac{i \delta}{A}\right)^{2}}+j \frac{i \delta}{A}\right)^{r k-1}\right\}-\left(\sqrt{1-\left(\frac{m \delta}{A}\right)^{2}}+j \frac{m \delta}{A}\right)^{r k-1}+(-1)^{r k-1}\right],
\end{gathered}
$$

which essentially is the same expression that (6.4) without $T_{s}$ in the denominator because it has been moved to the left term of equation (6.7). Additionally, the expression $2 \pi \tau / T_{s}$ in the exponential of $\mathcal{N}$ has been substituted by $\theta_{s}$. As $\tau \in\left[0, T_{s}\left[\right.\right.$, then $\theta_{s} \in[0,2 \pi[$. These small changes make $\mathcal{N}^{\prime}$ dimensionless and prevent it from being scaled by $T_{s}$. Therefore, similar to the continuous DF for $\mathrm{SSOD}, \mathcal{N}^{\prime}$ tends to $(-1,0)$ as $m$ increases, regardless of the sampling period. Additionally, to homogenize the notation in the stability condition (6.7), the frequency $\omega$ will be expressed in term of $r$ and the sampling frequency $\omega_{s}$ :

$$
\frac{G_{o l}\left(j \frac{\omega_{s}}{r}\right)}{T_{s}} \neq \frac{-1}{\mathcal{N}^{\prime}\left(\delta / A, r, \theta_{s}\right)} ; \quad \forall r \in \mathbb{Z}, r_{\text {min }}<r<r_{\text {max }}
$$

Even though the frequency $\omega$ does not appear explicitly in the equation (6.9), it is hidden within the ratio $r=T_{o} / T_{s}=\omega_{s} / \omega_{o}$ because each evaluated frequency $\omega$ is a candidate to become the oscillation frequency $\omega_{o}$. Therefore, as both sides of the equation (6.9) depend on the evaluated frequency it is important to know the range of $\omega$ where this condition must be evaluated to check the existence of limit cycles. In this sense, it is worth noting that the traces of the negative inverse of the presented DF lie in the third quadrant of the polar plot, see Figure 6.3 , thus the frequencies to be evaluated must be those for which $G_{o l}(j \omega)$ lie in this quadrant too. Even if the range of frequencies placed in the third quadrant is very wide, it is not necessary to check all of them. In the lines below the guidelines about the calculation of this range and the stability analysis are given.

As it has been commented before, the DF traces lie in the third quadrant. Thus, considering a given sampling frequency $\omega_{s}$, the frequencies in this quadrant that make $r$ an integer should be evaluated. The minimum value of $r$ will provide the highest frequency placed within the third quadrant. It is known that the crossover phase frequency $\omega_{c p}$ corresponds to a point of $G_{o l}$ over the real axis between the second and third quadrants, therefore, using this frequency, the minimum value of $r$ can be easily determined by:

$$
r_{\min }=\left\lceil\frac{\omega_{s}}{\omega_{c p}}\right\rceil
$$

The maximum value of $r$ can be also established by analyzing the shape of the DF traces under study. It is worth noting that, for a given sampling frequency, higher values of $r$ correspond 


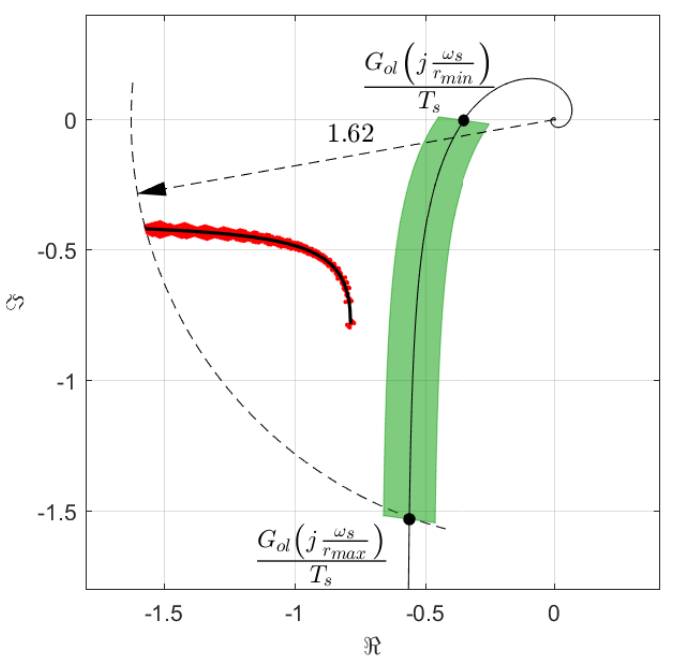

Figure 6.6: Graphical representation of the evaluated range of frequencies.

to lower frequencies $\omega$. The increment of $r$ also results in traces of $\mathcal{N}^{\prime}$ more and more similar to the continuous DF traces for the SSOD, which lie in a well defined zone in the third quadrant. To study the possible intersection between $-1 / \mathcal{N}^{\prime}$ and $G_{o l} / T_{s}$ there is no need to evaluate points beyond the extension of these traces, whose further point from the origin in the Nyquist diagram is at a distance of 1.62 units. Then, the point of $G_{o l}(s) / T_{s}$ with modulus equal to 1.62 determines the lowest frequency that must be evaluated to check intersection.

The previous ideas are illustrated in Figure 6.6. The negative inverse of the sampled DF with a high value of $r$ is represented in red, which is very similar to the continuous DF of the SSOD, represented in black. This shows that the sampled DF asymptotically tends to the continuous DF as $r$ increases, and consequently the range of $r$ to study intersection can be bounded as commented before. It can also be seen how the further point of $-1 / \mathcal{N}^{\prime}$ defines the point of $G_{o l}$ with lowest frequency that could intercept the negative inverse of DF: $\left|G_{o l}(s) / T_{s}\right|=1.62$. This point determines the maximum value of $r$. The range of frequencies that need to be evaluated to check intersection has been highlighted in green.

Analytically, the maximum value for $r$ can be easily obtained with:

$$
r_{\max }=\left\lceil\frac{\omega_{s}}{\omega_{c g}^{\prime}}\right\rceil
$$

where $\omega_{c g}^{\prime}$ is the frequency where the open-loop transfer function has the maximum modulus of the DF trace:

$$
\left\|\frac{G_{o l}\left(j \omega_{c g}^{\prime}\right)}{T_{s}}\right\|=1.62
$$

To clarify the stability condition (6.9) and the minimum and maximum values of $r$ the following example is introduced. 


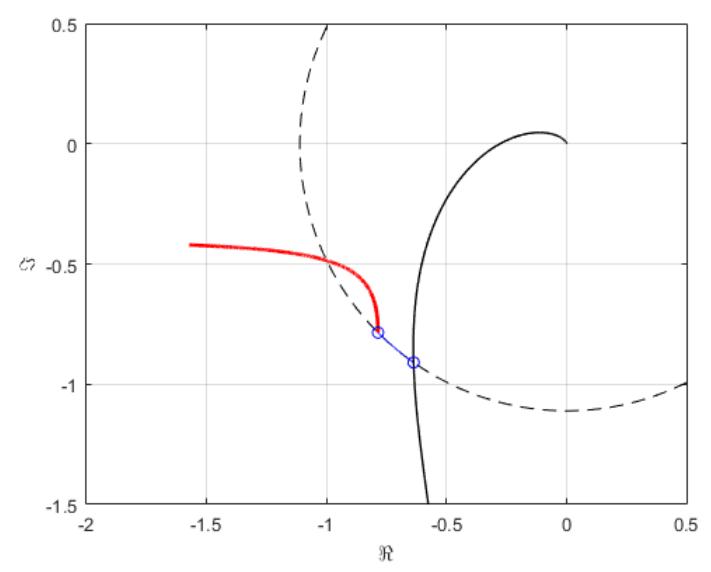

Figure 6.7: $G_{o l}(j \omega)$ and $-1 / \mathcal{N}$ for the continuous case. The presented PI avoids limit cycle oscillations in this case.

Example 19. Consider a process with transfer function:

$$
G(s)=\frac{1}{(s+1)^{3}} .
$$

A PI controller is tuned according to [56], where a tuning method is presented to prevent limit cycle oscillations in the control scheme in Figure 4.1. For that the method avoids the intersection between the open-loop transfer function and the negative inverse of the continuous DF of the SSOD sampler by assuring a minimum phase margin between $G_{o l}(j \omega)$ and the critical point of $-1 / \mathcal{N}$, which is set to $\Phi_{m, S S O D}=10^{\circ}$, while fulfilling a minimum gain margin restriction, which is fixed to $\gamma_{c p} \geq 6 \mathrm{~dB}$. The obtained parameters are $K_{p}=1.35$ and $T_{i}=2.38$ and the representation of the open-loop transfer function with the traces of the inverse negative of the continuous DF in the Nyquist diagram that validates this controller in the continuous case is presented in Figure 6.7.

It is worth remarking that the continuous PI with the previous parameters assures the avoidance of limit cycle oscillations in the control scheme in Figure 4.1. The goal, however, is to analyze the existence of limit cycles when using these parameters in a discrete PI controller $C(z)$ in the control scheme in Figure 6.1. Consider the trapezoidal form of the PI with a sampling period $T_{s}=0.5$ seconds.

The minimum value of $r$ has been obtained according to equation (6.10) for which the crossover phase frequency of $G_{o l}(s) / T_{s}$ was calculated $\left(\omega_{c p}=1.11 \mathrm{rad} / \mathrm{sec}\right.$.) and the minimum value of $r$ results in $r_{\text {min }}=\left\lceil\omega_{s} / \omega_{c p}\right\rceil=\lceil 12.57 / 1.11\rceil=12$. Analogously, the crossover gain frequency of $G_{o l}(s) / 1.62 / T_{s}$ has been obtained $\left(\omega_{c g}^{\prime}=0.38 \mathrm{rad} / \mathrm{sec}\right.$.) and the equation (6.11) has been used to calculate the maximum bound of $r$, which results in $r_{\max }=\left\lceil\omega_{s} / \omega_{c p}^{\prime}\right\rceil=$ $\lceil 12.57 / 0.38\rceil=33$.

Figure 6.8 a shows the Nyquist diagram of $G_{o l}(s) / T_{s}$ and the traces of the negative inverse of the Sampled DF for the level $m=1$. The points corresponding to several values of $r$ between the 


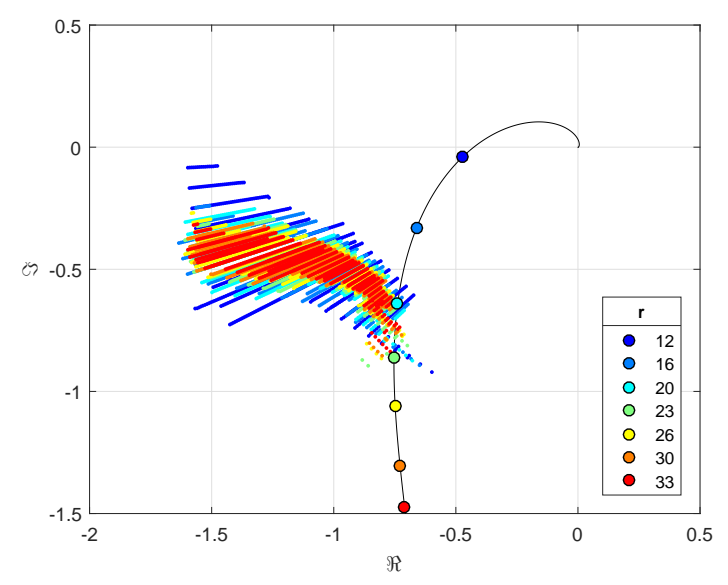

(a) Original $-1 / \mathcal{N}^{\prime}$ representation.

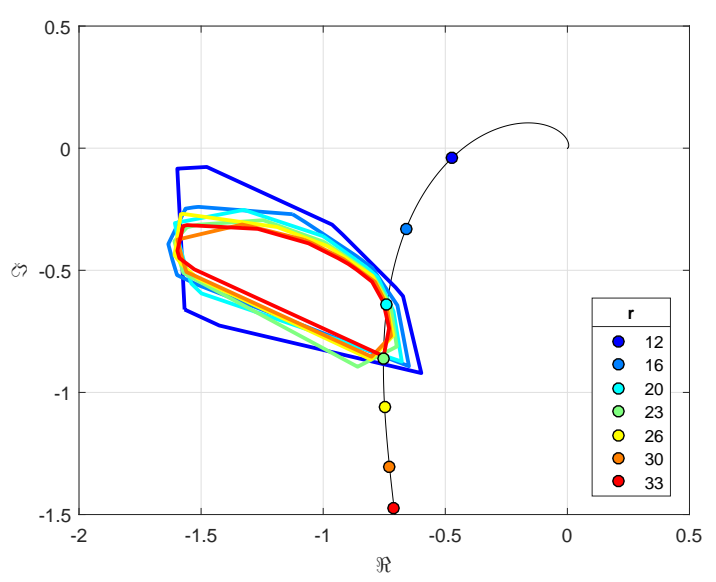

(b) $-1 / \mathcal{N}^{\prime}$ replaced by its respective convex hull.

Figure 6.8: Sampled DF for $m=1$ with $T_{s}=0.5$ considering different values of $r$.

bounds obtained above and their respective normalized Sampled DF traces, given by $-1 / \mathcal{N}^{\prime}(r)$, have been highlighted each in a different color. Then, according to condition (6.9), a limit cycle takes place if some point and trace highlighted with the same color intersect. Due to the complex shape of the traces, which overlap one to another, the previous condition is difficult to be evaluated from Figure 6.8a. A more clear representation is obtained by substituting the traces by their convex hull as in Figure 6.8b. Now the verification of the stability condition is easier: if $G_{o l}\left(j \omega_{s} / r\right) / T_{s}$ lies within the convex hull that contains $-1 / \mathcal{N}^{\prime}(r)$, the system could present limit cycle oscillations. In this case, $G_{o l}\left(j \omega_{s} / 20\right)$ is placed within the convex hull of $-1 / \mathcal{N}^{\prime}(20)$, therefore, a limit cycle oscillation could exist for $r=20$. The system has been tested in simulation with a SSOD sampler with $\delta=0.1$. The results from the simulation are presented in Figure 6.9, where the controlled output and the control action temporal responses to a unitary step change in the reference and disturbance inputs are depicted considering both the discrete and the continuous controllers. As can be seen, no oscillations are observed in the response of the continuous controller since the limit cycles are avoided by the tuning method. On the other hand, the response with the discrete controller presents limit cycle oscillations, whose frequency has been measured to be $\omega_{s} / 20$, which corresponds to the point of the openloop transfer function placed within its convex hull in Figure 6.8b. This example shows how the discrete version of a stable continuous controller could induce limit cycle oscillations in the control loop. This fact stress the importance of the analysis presented in this chapter.

\subsubsection{Robustness measure}

In Example 19 it can be noted that only branches with $m=1$ have been considered. As can be seen in Figure 6.3, no matter the value of $r$, the branches of $-1 / \mathcal{N}^{\prime}(r)$ shrink and move near to the real axis as $m$ increases. Taking this into account, the shape of $G_{o l}(s) / T_{s}$ obtained with a PI controller for most of the process models is such that the distance between $G_{o l}(s) / T_{s}$ and $-1 / \mathcal{N}^{\prime}$ increases with $m$. Therefore, the non intersection with the branches of $m=1$ guarantees 

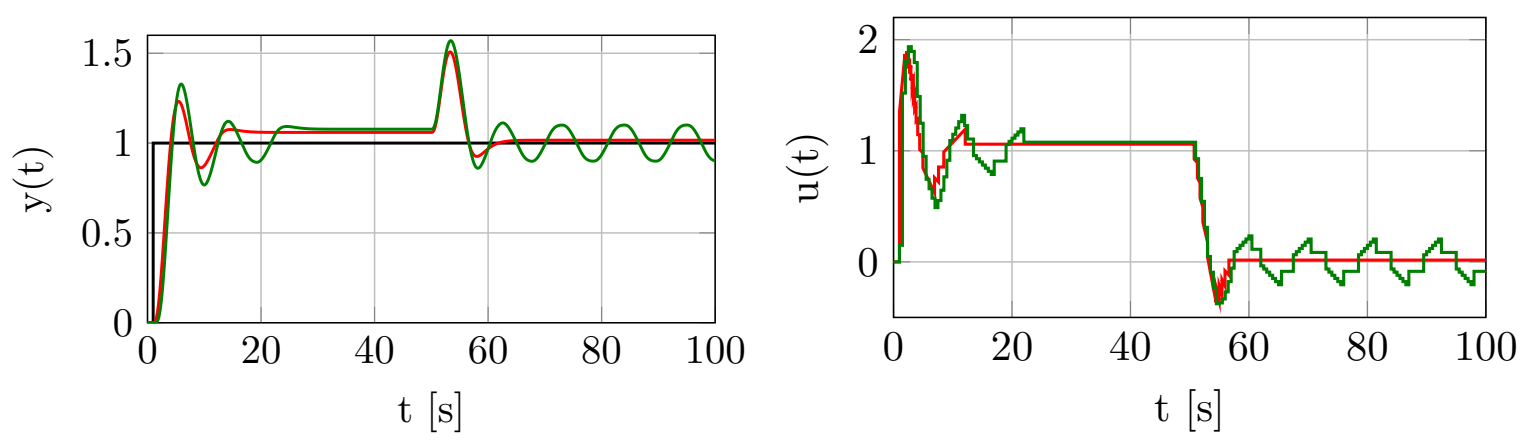

Figure 6.9: Controlled output and control action temporal response to a unitary step change in the reference and disturbance inputs with the discrete (green) and continuous (red) controller. The limit cycle predicted from the Sampled DF analysis can be observed.

no intersections for $m>1$. This fact was pointed out in [56] for the case of continuous PI and it would be demonstrated experimentally in the next section that this affirmation also holds for the sampled case. Consequently, only the branches of $m=1$ need to be considered to define a robust margin to avoid limit cycles.

Under the previous assumption, and considering the stability condition given by equation (6.9), a very simple robustness measure can be defined as the minimum distance between a frequency response point in the Nyquist diagram and its respective convex hull containing the traces for a given value of $r$. This measure will be called $D_{c h}$, and it can be formally defined as:

$$
D_{c h}=\min _{r_{\text {min }} \leq r \leq r_{\max }}\left(\operatorname{dist}\left(G_{o l}\left(j \omega_{s} / r\right) / T_{s}, C H\left(-1 / \mathcal{N}^{\prime}(r)\right)\right)\right),
$$

where $\operatorname{dist}(\cdot)$ denotes the Euclidean distance and $C H(\cdot)$ refers to the convex hull.

The following example illustrates the use of the proposed margin for measuring the robustness to limit cycle oscillation.

Example 20. Consider a process whose transfer function is defined by:

$$
G(s)=\frac{1}{(s+1)^{5}} .
$$

A PI controller has been tuned using AMIGO tuning rule [4] obtaining $K_{p}=0.2564$ and $T_{i}=2.891$. This method has been chosen because it has been proven that provides good robustness capabilities against limit cycle oscillations induced by the SSOD quantification, [44]. A sampling period $T_{s}=1.9$ has been selected for the discrete implementation of the controller, which corresponds to a tenth of the rise time of the continuous response.

Once the discrete controller has been implemented in its trapezoidal form, the robustness analysis as described previously has been performed. The convex hulls and the evaluated frequency points for different values of $r$ have been represented in Figure 6.10a. Circles representing the minimum distances from each frequency point to its respective convex hull are also shown in 


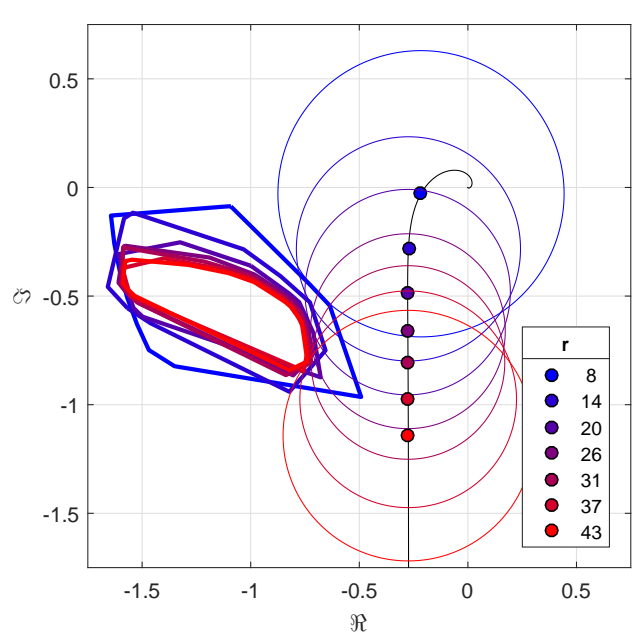

(a) All the distances to convex hull.

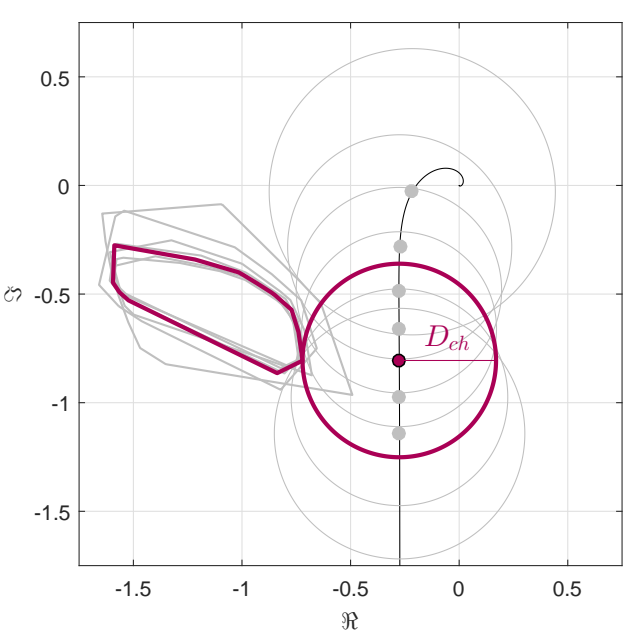

(b) $D_{c h}$ highlighted.

Figure 6.10: Representation of the distances from the evaluated points to their respective convex hull and detail of $D_{c h}$ for the studied system.

the figure. From those distances the minimum, which has been named $D_{c h}$, is the one that corresponds to $r=31$. As distinguishing it from all the convex hulls and circles from Figure 6.10a is not quite clear, it has been highlighted in Figure 6.10b. In this case the convex hull and the frequency in which $D_{c h}$ is obtained has been highlighted while the other distances, which are greater than $D_{c h}$, have been represented in gray.

Therefore, as for this example a value $D_{c h}=0.45$ has been obtained the system avoids limit cycle oscillations. To prove that, the system has been tested in simulation with a step change in the reference and disturbance inputs. The SSOD sampler used has a quantization level $\delta=0.1$. In Figure 6.11, the controlled output and control action temporal responses to a unitary step change in the reference and disturbance have been presented considering a continuous controller (in red) and a discrete controller (in green). As it can be seen, the system presents a smooth response and does not present limit cycle oscillations, as predicted by the robustness analysis, while not degrading significantly the performance provided by the continuous controller.

\subsection{Robustness of continuous tuning rules}

To illustrate the usefulness of the presented margin, it has been applied to study the robustness against limit cycle oscillations induced by the quantification and sampling of the SSOD. The discrete controller is implemented in its trapezoidal form as shown in Figure 6.2. The tuning methods for this study are Ziegler-Nichols [79], AMIGO [4], One-Third rule [27] and SIMC [68]. PI controllers have been tuned for the batch of models presented in (6.14), which describe a wide range of behaviors that can be found in real systems. The dynamic responses of the models in the batch have been approximated by First Order Plus Time Delay (FOPTD) models to obtain the parameters of their respective controller. 

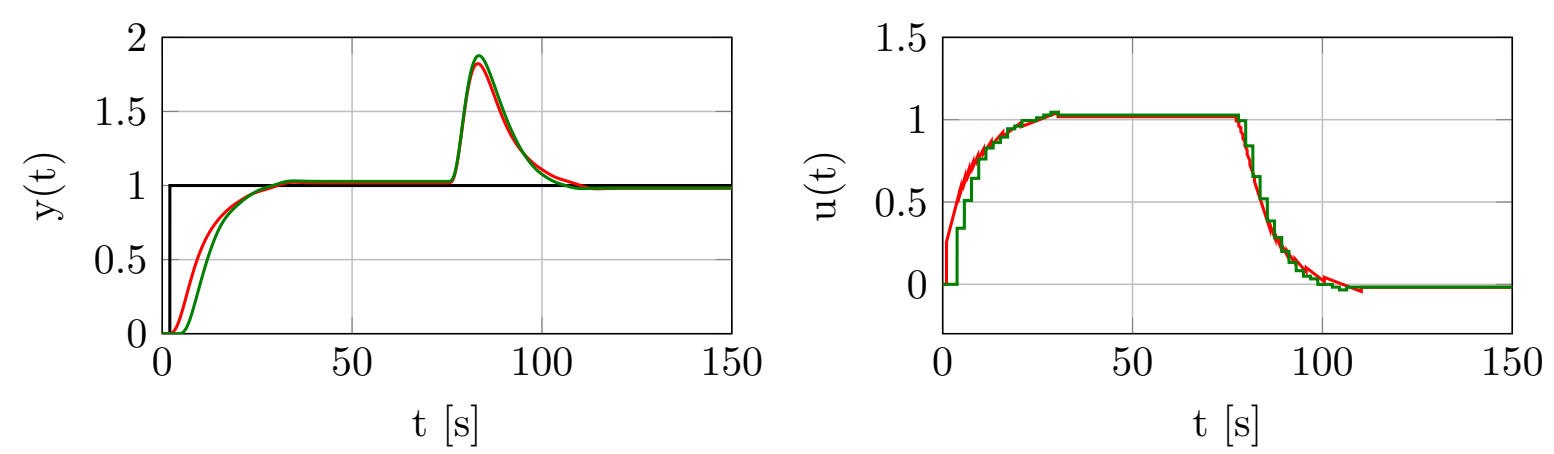

Figure 6.11: Controlled output and control action temporal response to a unitary step change in the reference and disturbance inputs with the discrete (green) and continuous (red) controller. The absence of limit cycle oscillations predicted by the Sampled DF analysis can be observed.

$$
\begin{aligned}
& G(s)=\frac{e^{-s}}{(T s+1)^{2}}, \\
& T=0.01,0.02,0.05,0.1,0.2,0.3,0.5,0.7,1, \\
& \quad 1.3,1.5,2,4,6,8,10,20,50,100,200,500
\end{aligned}
$$

$$
\begin{aligned}
& G(s)=\frac{1}{(s+1)(T s+1)^{2}}, \\
& T=0.05,0.1,0.2,0.5,2,5,10
\end{aligned}
$$

$$
\begin{aligned}
& G(s)=\frac{1}{(s+1)^{n},} \\
& n=3,4,5,6,7,8
\end{aligned}
$$$$
\begin{aligned}
& G(s)=\frac{1}{(s+1)(\alpha s+1)\left(\alpha^{2} s+1\right)\left(\alpha^{3} s+1\right)} \\
& \alpha=0.1,0.2,0.3,0.4,0.5,0.6,0.7,0.8,0.9
\end{aligned}
$$

$$
\begin{aligned}
& G(s)=\frac{T e^{-L_{1} s}}{\left(T_{1} s+1\right)(T s+1)}, \\
& T_{1}+L_{1}=1, \quad T=1,2,5,10
\end{aligned}
$$$$
L_{1}=0.01,0.02,0.05,0.1,0.3,0.5,0.7,0.9,1
$$$$
G(s)=\frac{1-\alpha s}{(s+1)^{3}},
$$$$
\alpha=0.1,0.2,0.3,0.4,0.5,0.6,0.7,0.8,0.9,1,1.1
$$

$G(s)=\frac{1}{(s+1)\left((s T)^{2}+1.4 s T+1\right)}$,

$T=0.1,0.2,0.3,0.4,0.5,0.6,0.7,0.8,0.9,1$ 


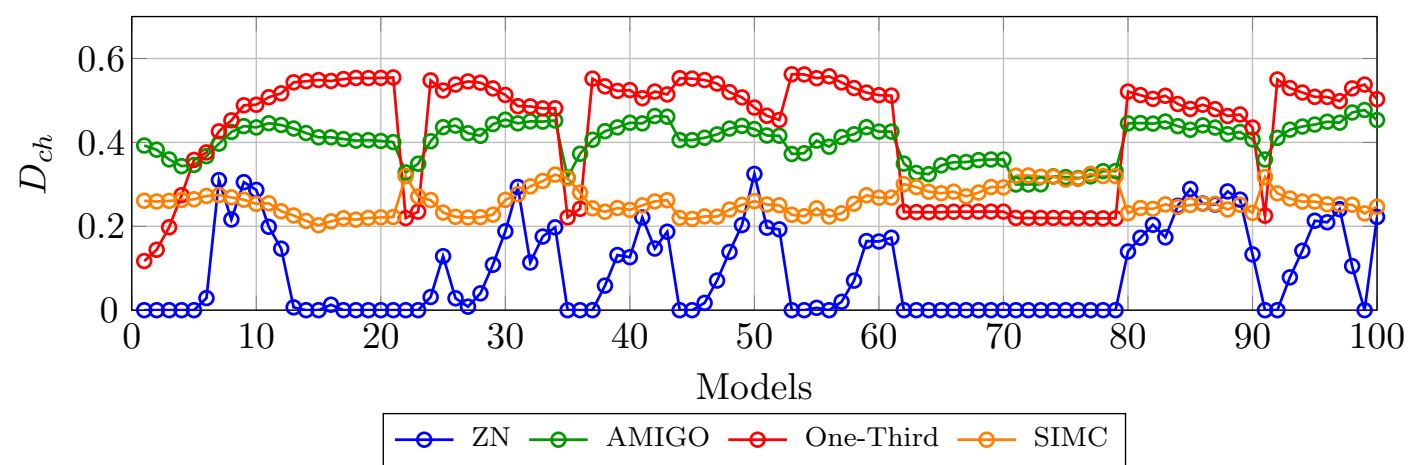

Figure 6.12: $D_{c h}$ for the batch of processes with different controllers considering $T_{s}=T_{r} / 10$.

To obtain the sampling period for each controller a simple procedure has been followed. The closed-loop response of each system with its controller in continuous has been obtained and its rise time $T_{r}$ has been measured. Then, the sampling period $T_{s}$ has been obtained as $T_{s}=T_{r} / 10$. With all the parameters obtained for the implementation of the discrete controller the robustness analysis as shown in the previous section has been done, obtaining the minimum distance to the convex hull $D_{c h}$ for each system and controller in the batch. The results are presented in Figure 6.12.

The first result that can be observed in this figure is that Ziegler-Nichols controllers struggle to avoid limit cycle oscillations. On the other hand, the other tuning methods offer different degrees of robustness but they manage to avoid limit cycle oscillations.

Regarding the methods that consistently avoid these oscillations, it can be seen that AMIGO and SIMC offer an uniform level of robustness while One-Third rule presents several bumps. These bumps are caused because the closest convex hull, with respect to which the $D_{c h}$ is measured, changes. For example, for process 60 the closest convex hull is the one that encloses $-1 / \mathcal{N}^{\prime}(21)$, but for the process 62 the closest convex hull is $-1 / \mathcal{N}^{\prime}(3)$. The case of the process 62 is illustrated in Figure 6.13, where it can be seen how the convex hull of $-1 / \mathcal{N}^{\prime}(3)$ is larger than the rest, overtaking all of them and, therefore, defining $D_{c h}$. However, this situation is not desirable in practice since having only 3 samples per period could be insufficient for control purposes. Nevertheless, despite the presence of these extreme cases, One-Third rule consistently avoids limit cycle oscillations and it provides some of the highest robustness measures.

A second robustness analysis has been performed with a more conservative approach considering $T_{s}=T_{r} / 20$. The obtained results are presented in Figure 6.14. As in the precedent case study, controllers tuned with Ziegler-Nichols method offer the lowest robustness in general lines, conducing most controllers to limit cycle oscillations. The other methods under study offer similar characteristics than with the previous sampling frequency. AMIGO and SIMC offer a more uniform level of robustness than the One-Third rule, which still presents some bumps in the measure $D_{c h}$ even if the total amount has diminished.

In Figure 6.15, the difference in the robustness measure $D_{c h}$ produced by the increase of the sampling frequency is presented. In this figure, it can be seen a behavior that matches with the 


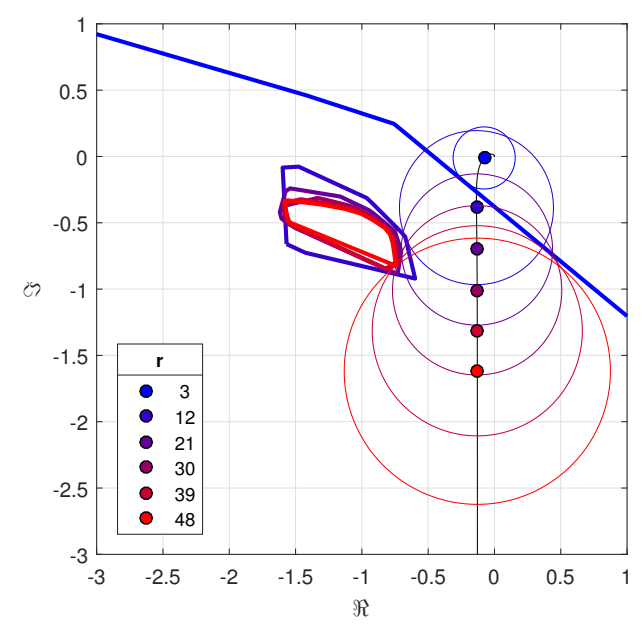

Figure 6.13: Some convex hull plots for process 62 . The convex hull of $-1 / \mathcal{N}^{\prime}(3)$ overtakes the rest, defining $D_{c h}$.

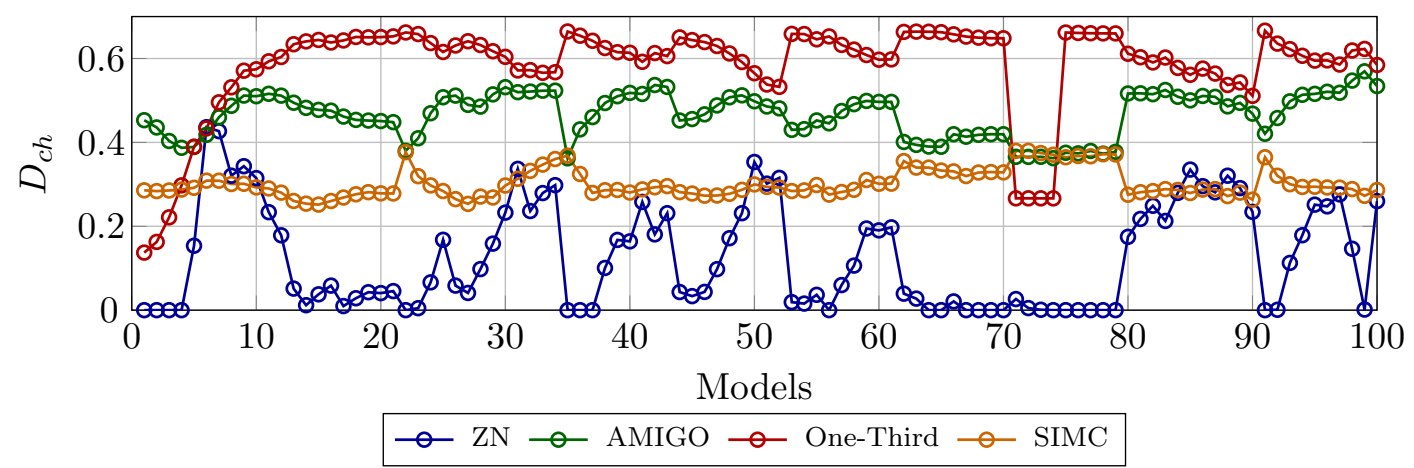

Figure 6.14: $D_{c h}$ for the batch of processes with different controllers considering $T_{s}=T_{r} / 20$.

general principles of the discrete control, the robustness increases with the sampling frequency. In all the studied processes and methods in the batch the robustness increases with the sampling frequency, being this rise greater for the One-Third rule. This tuning rule also presents a boost in the robustness for some processes, which are produced by avoiding some of the bumps in the robustness presented in Figure 6.12. As a consequence of increasing the sampling frequency, the value $r_{\min }$ has risen, avoiding large convex hull of undesirable situations, as the presented in Figure 6.13. The Ziegler-Nichols tuning rule also presents some bumps produced by a situation similar to the previously described.

This evaluation of some of the most used tuning rules designed for continuous processes reveals that Ziegler-Nichols tuning rule with the sampling criteria followed is not recommendable. In addition, it can be noted that the rest of tuning rules provide some degree of robustness against limit cycle oscillations and some interesting casuistic as the robustness bumps.

Remark 4. In general, a universally valid value of $D_{c h}$ that assures good behavior of the closed loop response is not known. However, values of $D_{c h}$ in the order of 0.2-0.6 have been obtained 


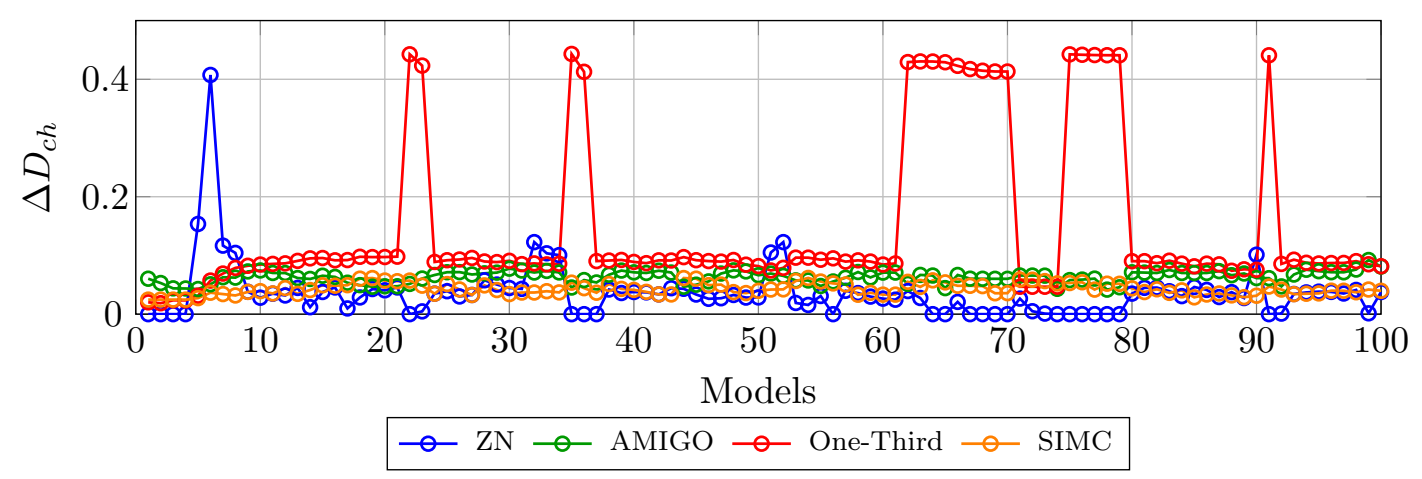

Figure 6.15: Variation of $D_{c h}$ produced by an increase in the sampling frequency.

from the previous study for the batch of processes with different tuning methods. Because some considered methods for tuning the PI provide acceptable temporal responses, even in a quasioptimal sense as in the case of AMIGO method, then values of $D_{c h}$ in this range could be considered reasonable to obtain good close loop performances.

\subsubsection{Effect of sampling period on the performance}

In general, discrete controllers have inferior performance over continuous control systems. This is sometimes explained due to the fact that sampled signals have less information than continuous signals. In order to study the effect of the sampling time on the performance of the discrete event-based control loop under study, the index $I A E^{*}$ has been used, which is defined as:

$$
I A E^{*}=\frac{\overline{I A E}}{I A E_{c}},
$$

where $\overline{I A E}$ and $I A E_{c}$ are the $I A E$ indexes of the system response to a step-like disturbance with the discrete event-based controller and the continuous controller respectively. The study has been conducted for the controllers obtained in the previous section considering three different sampling rates: $T_{s}=T_{r} / 20, T_{s}=T_{r} / 10$ and $T_{s}=T_{r} / 3$. The results are presented in Figure 6.16 .

It can be seen that it does not exist a great difference in performance between the sampling periods $T_{s}=T_{r} / 10$ and $T_{s}=T_{r} / 20$, despite the fact that in those cases a significant difference in robustness was observed in Figure 6.15. In addition, for a sampling period $T_{s}=T_{r} / 3$ the performance decreases in almost every case with regard to the other sampling rates. This downgrade of the sampling frequency not only worsens the performance of the system, but it has also been observed a significant loss of robustness. In fact, in Figure 6.16 most of the values of $I A E^{*}$ for this sampling rate have been omitted since their respective $D_{c h}$ values were 0 .

The robustness measure for the sampling rate $T_{s}=T_{r} / 3$ is presented in Figure 6.17, where it can be seen that most of the controllers operating under this sampling rate will present an oscillatory behavior since $D_{c h}=0$. For those controllers that avoid limit cycle oscillations, it 

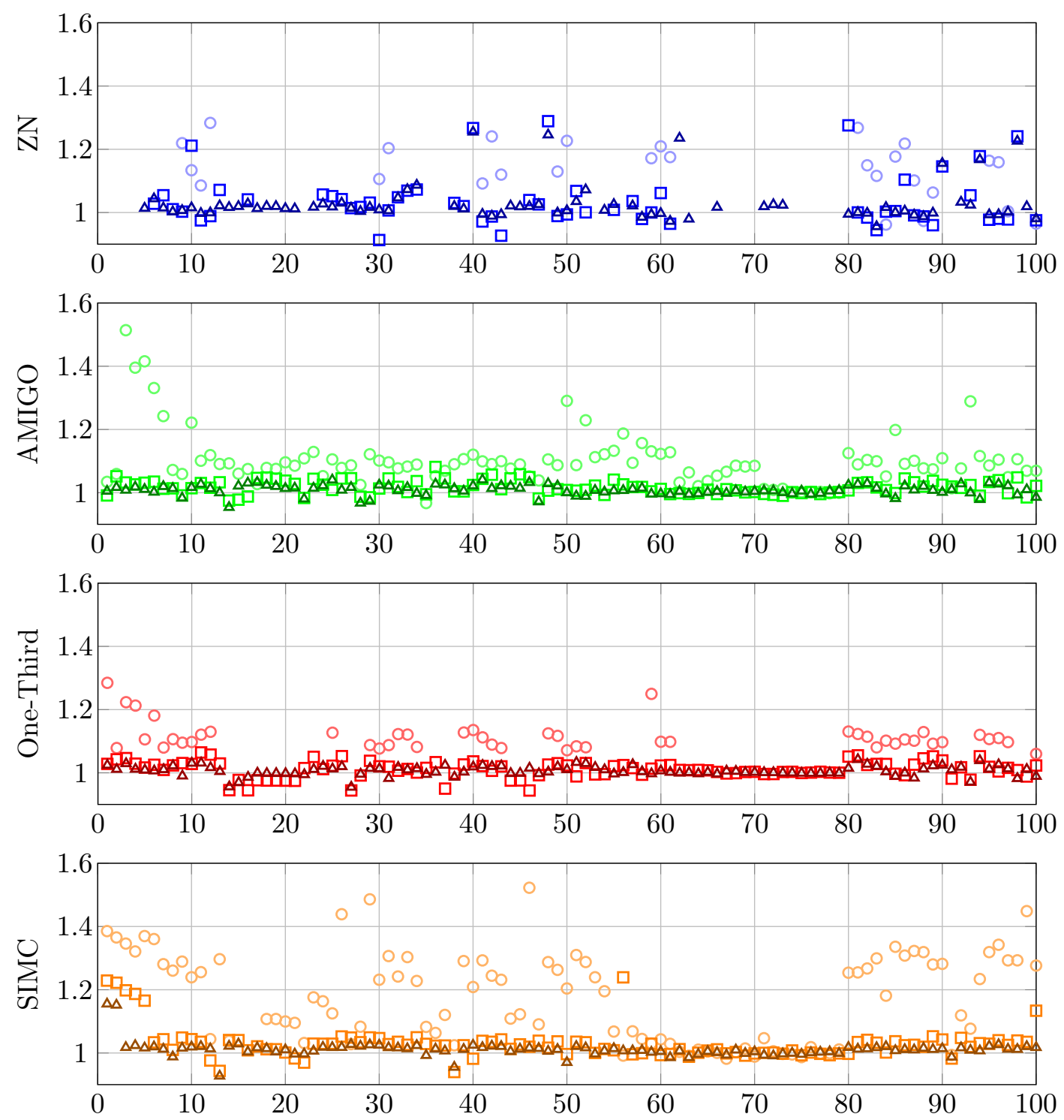

Figure 6.16: $I A E^{*}$ for the batch of processes with different controllers (circles: $T_{s}=T_{r} / 3$, squares: $T_{s}=T_{r} / 10$, triangles: $\left.T_{s}=T_{r} / 20\right)$. 


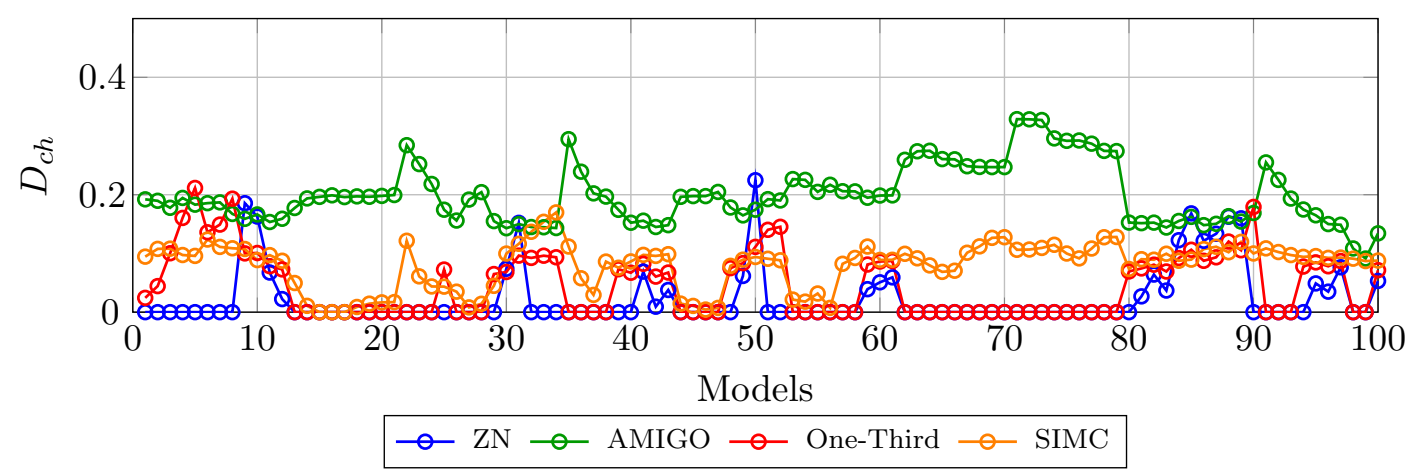

Figure 6.17: $D_{c h}$ for the batch of processes with different controllers considering $T_{s}=T_{r} / 3$.

can be observed a significant loss of robustness comparing the values of $D_{c h}$ presented in this figure with the values obtained for $T_{s}=T_{r} / 10$ and $T_{s}=T_{r} / 20$, which were presented in Figures 6.12 and 6.14 respectively. In summary, it can be concluded that the sampling rate has a strong influence in both the robustness and the performance which are improved as the sampling rate rise.

\subsubsection{Influence of model uncertainties on $D_{c h}$}

Regarding the uncertainties in the parameters of the plants, it is clear the higher the value of $D_{c h}$ the more admissible modeling error or variations in the parameters before limit cycle oscillation take place. In general, the uncertainties in the model can effect both the module and the phase of the $G_{o l}$. The uncertainties affecting the modules of $G_{o l}$, e.g. those in the process gain, only produce a radial displacement in $G_{o l}(s) / T_{s}$ which expands or shrinks as the gain increases or decreases respectively. Therefore, as convex hulls obtained for the calculation of $D_{c h}$ remain invariant in this case, an increment in the process gain implies a reduction on $D_{c h}$ because the critical points are getting closer to its respective convex hull.

The uncertainties that affect the phase of the process will modify the crossover phase frequency, modifying the value of $r_{\text {min }}$. In addition, this will produce a turn of $G_{o l}(s) / T_{s}$ in the Nyquist diagram, approaching or separating the critical points from their respective convex hull. Phase increment could take place if the time delay rises, which would add $\omega L$ radiants to the phase of $G_{o l}(s) / T_{s}$, where $L$ is the delay. In that case, the robustness measure $D_{c h}$ would decrease since the values of $r$ to evaluate would be lower, increasing the dispersion of the Sampled DF traces, and the turn on $G_{o l}(s) / T_{s}$ would approach the critical points to those convex hulls. Other variations on the open-loop transfer function could be induced by fluctuations on model parameters such as zeros and poles. Beyond the general considerations aforementioned, the admissible uncertainties in each parameter for a concrete model must be studied by an ad-hoc analysis. 


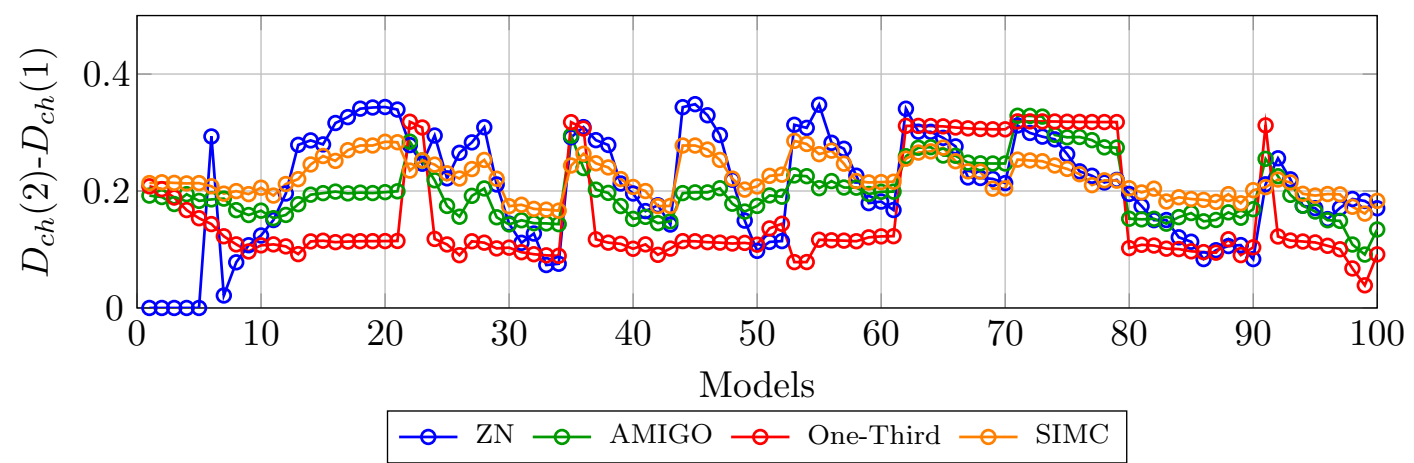

Figure 6.18: Difference between the robustness against oscillations of type $m=2$ and of type $m=1$.

\subsubsection{Multi-leveled oscillations}

The presented robustness study based on the proposed measure $D_{c h}$ is substantiated on the assumption that by avoiding single-leveled oscillations (those with $m=1$ ), multi-leveled oscillations $(m>1)$ will also be avoided. This fact was pointed out in [56] for the continuous case, but for the sampled case this study has not been addressed yet.

The definition of $D_{c h}$ can be adapted to contemplate multi-leveled oscillations by considering only the traces of $-1 / \mathcal{N}^{\prime}$ of the level under study in equation (6.13). Therefore, the robustness against, for instance, oscillations of two levels, can be determined by considering the cases where $m=2$ in equation (6.8) and obtaining their convex hulls, which will then be used to calculate the minimum distance to their respective critical point as explained in previous sections.

This variation of $D_{c h}$ has been used to evaluate the robustness against two-leveled oscillations of processes in the batch presented in (2.15) with the controllers tuned with Ziegler-Nichols, AMIGO, One-Third and SIMC tuning rules. The difference between the robustness against limit cycle oscillations of two levels $D_{c h}(2)$ and single-leveled $D_{c h}(1)$ is presented in Figure 6.18. The measure $D_{c h}(1)$ is the same that the presented in Figure 6.12.

From Figure 6.18 it can be observed an increase in the robustness in all cases. The unique cases where there is not an increase is on the first five processes tuned with Ziegler-Nichols method, which presented an oscillatory behavior, and would also present it for $m=2$. In the other cases, a generalized increase in the robustness margin is observed, proving that by avoiding single-leveled oscillations, multi-leveled oscillations are also avoided.

\subsection{Conclusions}

In this chapter, an approach to study the robustness of discrete event-based systems has been presented. The event generator under study is the Symmetric-Send-On-Delta quantifier and the controller has been implemented in a discrete fashion, which represents more accurately the current implementation of this kind of systems. 
To perform the robustness analysis the Describing Function technique has been used, obtaining the Sampling Describing Function of the studied non-linearity. The characteristics of this Sampled DF have been studied, which ultimately presents characteristics similar to the continuous DF.

From the obtained Sampled DF and the stability condition several modifications have been made to facilitate the robustness analysis. Firstly, the range to evaluate both the Sampled DF and the system under study have been bounded. Secondly, the scattered points resulting from the study have been grouped in a convex hull. Finally, a robustness measure has been established as the minimum of the distances to a convex hull. The validity of the approach has been tested through several examples.

Using this robustness measure some well known tuning rules have been evaluated, namely, Ziegler-Nichols, AMIGO, One-Third rule and SIMC. These tuning rules have been used to obtain the controllers for a wide batch of systems. To perform the discrete implementation the sampling period has been chosen based on the rise time of the continuous system response. The obtained results reveal that Ziegler-Nichols tuning method struggles to offer controllers that avoid limit cycle oscillations. The other rules offer controllers with different levels of robustness, but they avoid consistently the apparition of limit cycle oscillations. In addition, it has been proved that the robustness of the controllers increases with the sampling frequency, behavior that matches with the sampled control theory.

Despite the fact that some of the aforementioned tuning methods, e.g AMIGO, avoid the limit cycles oscillation for most of the systems included in the studied batch, these methods does not take into account the condition to avoid limit cycles and it must be checked a posteriori. The robustness measure presented in this paper can be useful to define new tuning methods which consider the restriction on $D_{c h}$ as a design requirement: $D_{c h}>D_{c h r}$ where $D_{c h r}>0$ represents the required value of this robustness margin. Obviously, these new methods must also guarantee a good overall performance of the control systems.

Although the study presented in this chapter considers the SSOD sampler, the sampled DF approach could be also applied for analyzing the robustness to limit cycles when more general sampling strategies such as RQH are used, or even in the case of asymmetric multi-level relays. 



\title{
Implementation and experimental evaluation of SSOD and RQH sampling strategies for EBC
}

\begin{abstract}
In this chapter, the implementation of SSOD and RQH sampling strategies under the principles stated in standard IEC 61499 is addressed. Afterwards, an experimental distributed control network case is presented, in which these sampling strategies will be used. This framework will be used to validate experimentally the validity of the sampling strategies as well as the control algorithms and tuning rules proposed in previous chapters.
\end{abstract}

\subsection{Introduction}

During the last decade numerous investigations have been carried out about Event-Based Control (EBC) of continuous systems. The EBC pursuits two main objectives: (1) reducing the amount of information needed to perform the closed-loop control and (2) decreasing the mean computational cost that control algorithms require.

In Event-Based Controllers, the control algorithm is only executed after the occurrence of asynchronous events that indicate a significant change in the system state, whereas in the conventional time-based controllers, whose use is more extended, the control algorithm is executed periodically. Some examples of asynchronous event generation are given in [19], among them, one of the easiest to implement is the generation of events whenever the error signal surpasses certain thresholds.

Among the earliest contributions to event based PID control it can be found the seminal paper of Årzén [3], where the main objective was to reduce the CPU usage of the control systems without significantly disturbing the performance of the control loop. Some posterior works have 
been focused on resolving some issues of the initial proposal, mainly, related with the calculus of the integral time by Durand [22, 23] and Vasyutynskyy [76, 77].

In his work, Årzén proposes a periodic call to the control algorithm, not being periodic its execution, i.e. the control action is updated only when needed. The algorithm includes an event generation logic based on the difference between the actual error and the error used for the last actualization of the control action. If this difference is greater than a given threshold, then the controller output is recalculated. Moreover, the time without refreshing the control action is also limited.

The event generation technique used by Årzén relative to the error signal is known as Send-On-Delta (SOD) and it has been widely used because of its simplicity. This technique has derived into more complex rules like the Symmetric-Send-On-Delta (SSOD) [10], which essentially maintains the same functioning that the SOD but adds an hysteresis of value Delta and it has fixed thresholds. Other works as [42] study the benefits of reducing the magnitude of this hysteresis, resulting in a non-linearity called Regular Quantification with Hysteresis (RQH), which presents benefits in the number of events generated while reducing some robustness requirements.

Several studies have been conducted to propose some tuning methods that take into account the effect of the SSOD sampling on the overall performance of the loop. Some works like [11] follow a more experimental approach based on classical control performance measures, others such as $[56,57,44]$ use some robustness measures defined specifically for SSOD to perform the tuning procedure. These robustness measures or the techniques used to develop them have also been used to evaluate the performance of RQH sampling [58, 42].

However, in the literature aforementioned, the effects caused by the implementation of such control loops on their performance is not studied deeply. Recently, some advances have been made in relation with event-based data acquisition systems [74]. The samplers that implement event generation techniques similar to the required by Årzén's algorithm, as that presented in [67], are very complex. Besides, most of the target industrial control devices that can implement $\mathrm{EBC}$ and benefit from its advantages usually operate in a periodic fashion, due to the standard in which they are implemented IEC 61131 [32], contradicting the asynchronous nature of EBC.

Nevertheless, other implementation options are available like IEC 61499 standard [80], which has been developed for programming distributed control systems. This standard introduces new concepts in comparison to its predecessor, mainly, whereas the IEC 61131 is based on scan cycles, the IEC 61499, whose goal is to favor the design of distributed and reconfigurable control applications, is based on the management and treatment of events. Hence, as the application frame of IEC 61499 standard and EBC is shared and the fields of study that are currently been studied complement each other, their combination will result in a symbiotic relationship between both areas.

Therefore, the standard IEC 61499 will be used to develop the implementation of EBC using SSOD and RQH sampling strategies. Then, with the obtained implementation of EBC, a distributed application in standard IEC 61499 will be configured to evaluate experimentally 
the performance and the robustness of several controller configurations presented in precedent chapters. To that end, several measures will be used to compare them and asses quantitatively their performance.

The chapter is organized as follows. In section 7.2 the principles of the industrial programming standard IEC 61499 are introduced. Section 7.3 details the design of the RQH function block in standard IEC 61499. In section 7.4, the elements that constitute the framework to validate the controllers are presented. The experimental validation of sampling strategies and controllers is presented in Section 7.5. Finally, the conclusions of this chapter are exposed.

\subsection{Principles of IEC 61499 programming standard}

It is important to remark that standard IEC 61499 appears as an answer to the necessity of implementing distributed applications in a standardized framework with ease, and not as a new programming methodology. Therefore, the focus of this standard revolves around the possibility of implementing applications deployed in several devices. The standard comprises several views or models of the system, each of them focusing on modeling different aspects.

The standard IEC 61499 is settled around the concept of Function Block (FB), which is the minimal functional unit of software with its own data structure that can be manipulated by one or more algorithms. In FBs interfaces coalesce data, which can have any type defined in IEC 61131, e.g. int or boolean, and events which regulate the execution control of the FB. These two types of data appear at the input and output interface of the FB. FBs are organized in types, each type having the same data structure, and can exist several instances of each type.

Depending on how FBs are formally defined, i.e. how the internal behavior is presented, they belong to different categories:

- A Basic Function Block is defined using a state-transition diagram called Execution Control Chart (ECC), in which algorithms are triggered as response to input events and output data and events are triggered as a consequence.

- Composite Function Blocks are defined by a network of FB instances. The behavior will depend on the FBs used and how are they connected.

- Service Interface Function Blocks permit to interact with external services from the interface of a FB. For example, they allow the communication between devices or reading the value of the inputs of a device.

FBs are gathered in a model that defines an IEC 61499 application, called Application Model. An application is defined in terms of behavior and structure by the instances of FB used and the interconnection of events and data between them, being this setup sufficient to regulate the behavior of the application. In this model the general behavior of the whole application is presented, regardless of the devices that will run the application. The FBs that implement some 
technical details, but they are not strictly part of the application behavior are not present in this model, e.g. FBs that allow communication between devices.

As it has been said, applications in this standard can be deployed in several devices and/or resources. The model that describes the devices, their resources, the networks and the connection between each of these elements is the System Model. This model describes the infrastructure of the system and allows assigning some properties, e.g. assigning IP addresses to devices for an Ethernet connection.

Once the elements are defined, each of the FBs that appears in the application model can be mapped to its device, assigning in this way who will be in charge of executing each element. To include the FBs necessary for example for the communication between devices that have been omitted in the Application Model, the standard provides an additional model called Device Model. In this model, the part of the application belonging to each device is presented, and here the additional FBs supporting the correct execution of the application are added.

The standard provides other models describing different aspects of the execution or the application, but for the scope of this work those models will not be explained in detail here.

\subsection{Development of RQH FB in IEC 61499}

One of the key aspects of standard IEC 61499 is the re-usability of the code. The development of applications is a proof of that because they are defined in terms of function blocks, which can be instantiated multiple times in different applications. Therefore, the RQH FB will not be designed to take into account device-specific properties, and it will only contain the quantization. Therefore, a Basic Function Block will be used.

The input-output relationship of the RQH quantization is defined in equation (2.1). From here the data that belong to the interface of the FB are extracted, being the signal $x(t)$, the quantization level $\delta$ and the hysteresis $h$ inputs to de FB, and $\bar{x}(t)$ its output. For notation issues the output of the quantization has been renamed as $y$. Note that the parameter $i$ is not listed among the input-output data, and it will be considered as an internal variable of the FB.

The input interface of the FB will be completed with an initialization event (INIT) and a request event (REQ). The INIT event will update the value of $\delta$ and $h$ and the REQ event only the value of the input $x$. The output interface will be composed of an initialization event (INITO), a confirmation event (CNF) and an additional event (EVT). In addition, a boolean variable Change is added to the data interface. The appearance of the final FB RQH_quant can be seen in Figure 7.1. The open-source program 4DIAC [1] has been used to model and develop the FBs, applications and system configuration used, and to deploy them on the physical resources.

Regarding the behavior of the FB, the values of the sampler will be taken at the initialization with INIT event, an event INITO will be sent, and the FB will remain in an Idle state waiting for requests. If while in this state another INIT event is sent, the FB will be reseted. If a sampling 


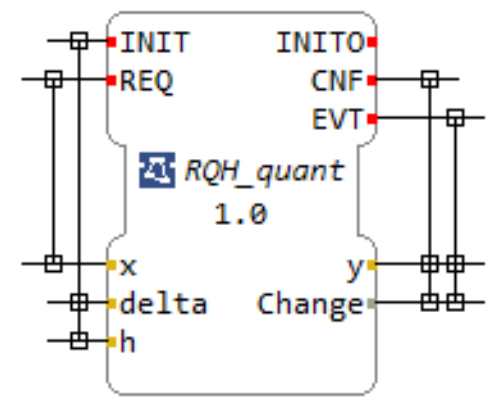

Figure 7.1: Interface of the FB RQH_quant.

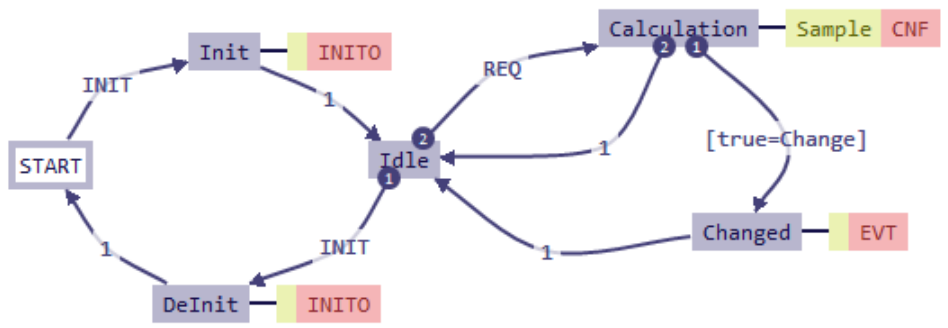

Figure 7.2: Execution Control Chart describing the internal behavior of the FB RQH_quant.

request (REQ) is received, the FB will compute the quantization presented in equation (2.1) and send a confirmation event (CNF). If a change in the output is detected, the boolean variable Change will became true and an event EVT will be sent. Either if a change is produced or not, the FB will return to its idle state. The ECC that describes this behavior is presented in Figure 7.2 , where it can be seen that the transition that evaluates if it has been produced a change in the system (true $=$ Change) has a higher priority than the return to the Idle state, denoted by a number at the origin of the transition, being this number the order in evaluating the transitions.

An example of the functioning of this FB is shown in Figure 7.3, where it has been presented in black solid line the signal to sample, in blue the theoretic output of the sampler and in red the output of the FB. Additionally, REQ (which are the same that CNF) and EVT events are also indicated with red and violet arrows respectively. Depending on the request frequency different behaviors can be obtained, if the frequency is selected high enough with regard to the process dynamics, a behavior similar to a continuous sampling can be obtained. If that frequency is lowered, as shown in Figure 7.3 a sampled non-linearity behavior as the shown in Chapter 6 will be obtained.

\subsection{Study framework}

Once the FB implementing the functionality of a $\mathrm{RQH}$ quantifier has been developed, the control implementation can be tackled. As it has been said, in IEC 61499 programming the applications are organized and regulated by an ensemble of FBs and their connections. These 


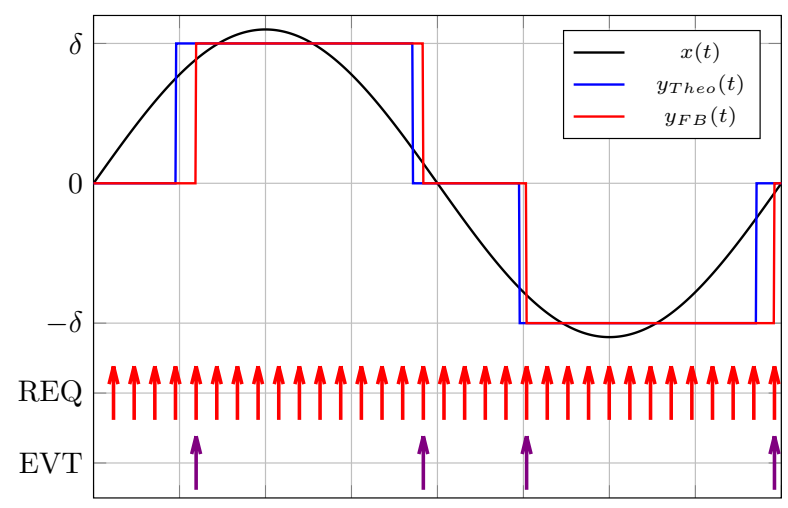

Figure 7.3: Example of the functioning of the RQH_quant FB. Signal to sample in solid black, theoretic result in solid blue, output of the FB in solid red, REQ events in red arrows and EVT events in violet arrows.

FBs are mapped to a certain device, which is responsible of providing the resources necessary for their execution, and their disposition is presented in the system model. In the next lines the experimental setup and its link to these implementation concepts is described.

The experimental setup chosen to validate the theoretical results presented in previous chapters consists of a laboratory scale system composed of two connected water tanks and a pump. The tanks are placed at different heights and connected between them with a pipe with a manual valve. The pump is placed in the lower tank and pours water into the upper tank. The goal is to control the level of liquid in the upper tank by actuating on the pump. The described system is depicted in Figure 7.4.

The upper tank counts with a level sensor which is connected to a BeableBone Black card, which is in charge of the data acquisition, and the pump is controlled with another BeagleBone Black which has a PWM signal as control output, whose power is increased by a specific card. Varying the duty rate of the PWM the pump can be regulated. A detailed view of the sensor and actuator units is included in Figure 7.5. Disturbances are introduced in the system by limiting the output flow with the manual valve in the pipe connecting both tanks.

These actuator and sensor units are connected to a $\mathrm{PC}$ that provides an user interface for displaying information about the current state of the system and allowing to modify the setpoint value through an Ethernet network. The sensor and actuator units and its link to the PC, which is done via a router, are shown in Figure 7.6. About the data flow over the network, the actuator unit receives data from the sensor unit and from the PC when a change on the set-point is introduced through the user interface, which also receives data of the measured signal and the control action applied to the process.

The presented framework is thus arranged in three different systems, namely, the sensor unit, the actuator unit and a user interface.These devices are presented in Figure 7.7 where it can be seen that they are connected to one another through an Ethernet network. Regarding to the application, each unit will develop some additional tasks to ensure its correct functioning: 


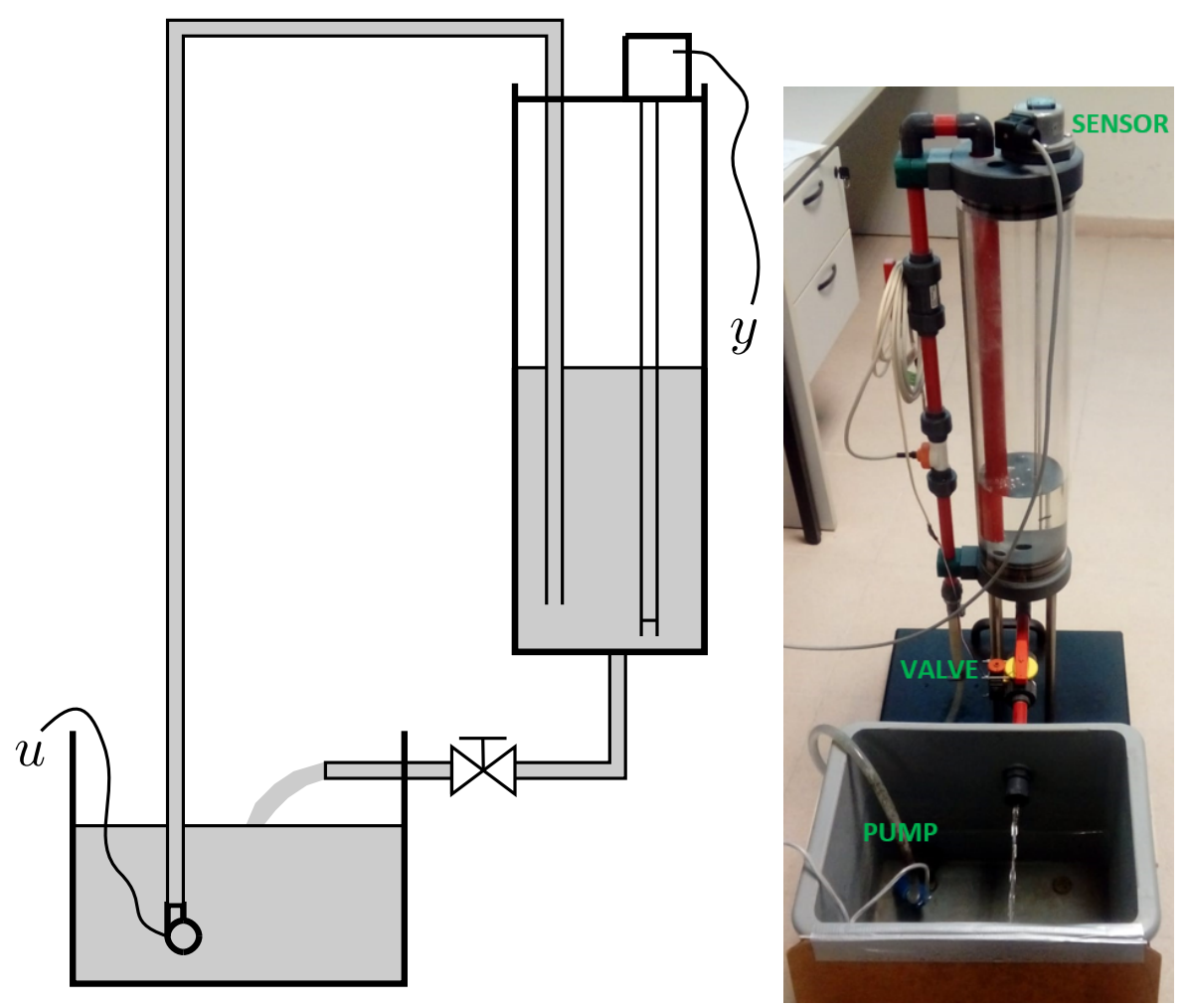

Figure 7.4: System with two connected tanks. Schematic representation and real system.
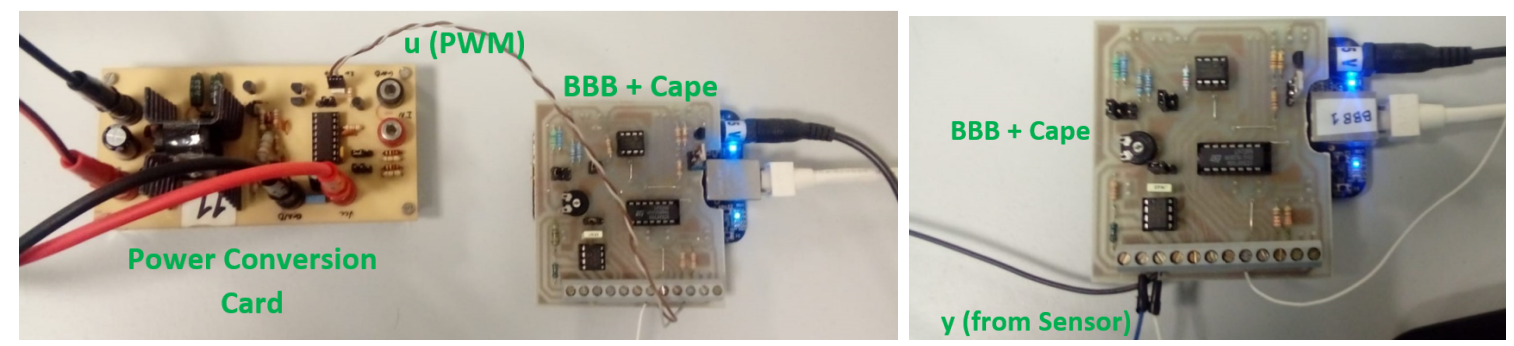

Figure 7.5: Detail of the actuator (left) and sensor (right) units. 


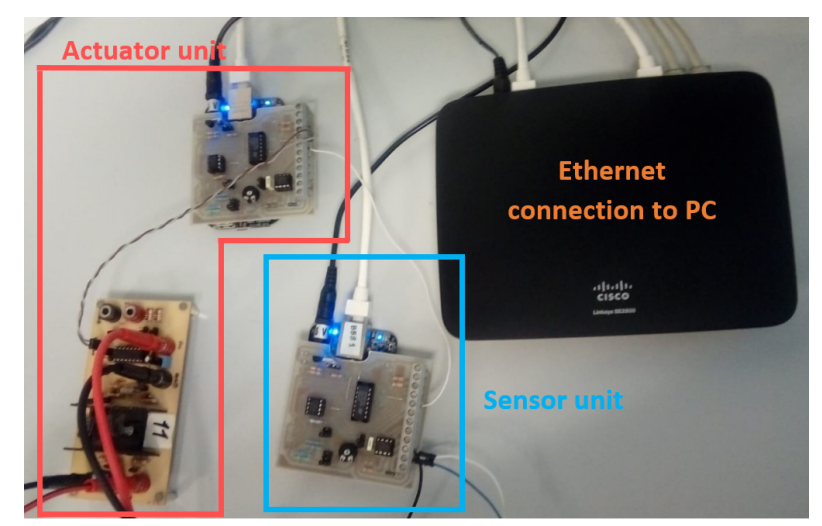

Figure 7.6: Actuator and sensor units. The connection between elements is supported via Ethernet.

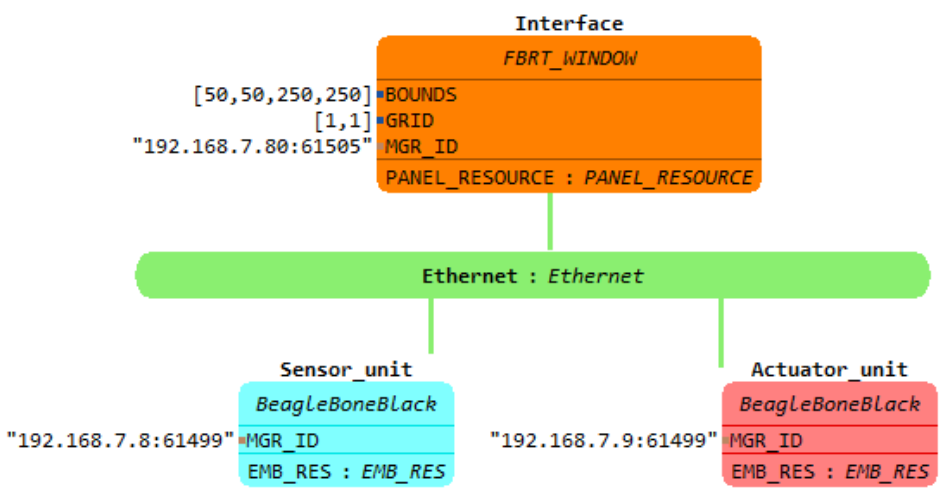

Figure 7.7: Devices of the study framework and their connection in the System Model.

1) the sensor unit, will be in charge of sampling, quantifying, and transmitting the measured signal 2) the actuator unit, will receive the measured signal, compute the control algorithms and modify the control output signal and 3) the interface management unit will provide information about the current state of the system and will allow regulating the set-point.

The general layout that will be used for the application model is presented in Figure 7.8, where it can be seen in a different color the FB mapped to each device. The data displayed in this figure can vary depending on the experiment being run, but the underlying ideas of a distributed control loop and how it is implemented in IEC 61499 are presented in this figure. It has to be noticed that even though the execution of the FBs entails a given execution period defined by the instance "Sampling period" of the E_CYCLE FB, this period has been chosen small enough $(200 \mathrm{~ms})$, in the cases where a continuous control is to be tested, with regard to the dynamic of the system, so the controller can be considered as a continuous one, such as it is assumed in previous chapters for its analysis.

It must be remarked that more FBs than the presented in the application model are necessary to implement a distributed application, which are modeled in the device model. These FBs are mainly communication-related FBs and their implementation is straightforward following the 


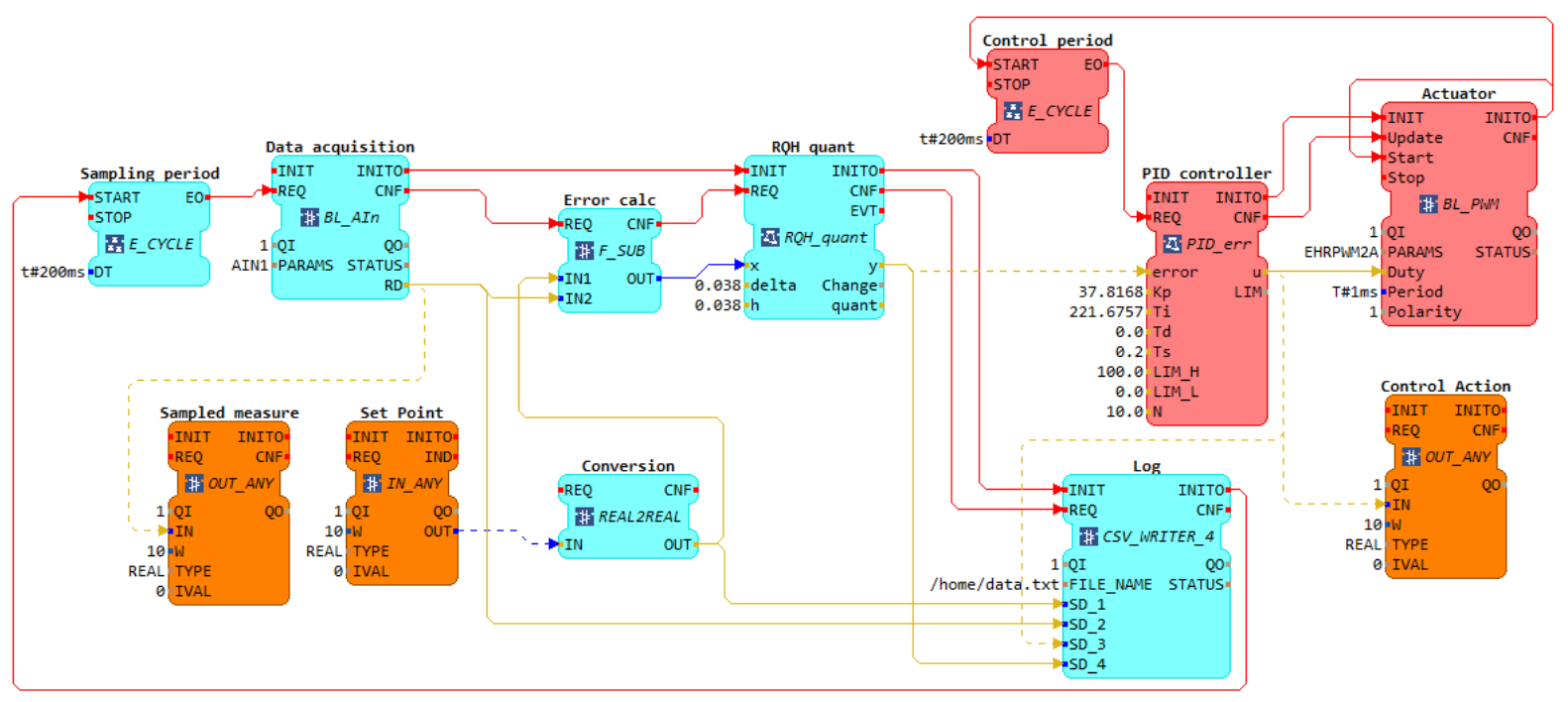

Figure 7.8: General layout of the Application Model of the study framework.

example tutorials in 4DIAC web page. For the concrete case under study the device models for each of the three devices are presented in Appendix E, in which these communication FBs are used to enable the communication between devices.

Additionally, in order to deploy any 4diac application the resource must contain and execute the runtime which supports the application called FORTE. Some pre-compiled versions of FORTE can be found on 4diac's web page [1] for different platforms which contain a basic set of FBs. However, as some custom FBs have been developed the runtime must be recompiled with the new additions. The cards and the $\mathrm{PC}$ are provided with this recompiled runtime.

\subsection{Experimental validation of EBC controllers}

Regarding the general application layout presented in Figure 7.8 several specific configurations can be set to validate the control situations presented in Chapters 2, 4 and 6 .

The system above presented has been identified using the step response method by the following SOPTD model:

$$
G(s)=\frac{0.0756 e^{-2 s}}{(143 s+1)(6 s+1)} \quad\left[\frac{V}{\%}\right] .
$$

which relates the duty cycle of the PWM signal driving the pump and the voltage in the output of the level sensor, that is related to the level of liquid in the tank through the ratio $250 \mathrm{~cm} / 9 \mathrm{~V}$.

This model describes the system dynamic around the middle of the level sensor operation range, that is, in a safe region of operation where there is no risk of overflowing. The measurement noise is mostly gathered in a band of $0.01 \mathrm{~V}(0.28 \mathrm{~cm}$ in tank level), which is assumed as the peak-to-peak amplitude of the noise. 


\begin{tabular}{c|c|c|c|c|c|c|c} 
& \multicolumn{2}{|c|}{ SLR } & \multicolumn{2}{c|}{ SHR } & \multirow{2}{*}{ AMIGO } & $\begin{array}{c}\text { Ziegler } \\
\text { Nichols }\end{array}$ & Tsypkin \\
\cline { 2 - 5 } & SSOD & RQH & SSOD & RQH & & \\
\hline $\mathbf{K}_{\mathbf{p}}$ & 157.37 & 157.37 & 37.82 & 93.76 & 45.96 & 142.05 & 52.51 \\
\hline $\mathbf{T}_{\mathbf{i}}$ & 44.76 & 44.76 & 221.68 & 107.76 & 80.36 & 36.11 & 90.76
\end{tabular}

Table 7.1: Parameters for the different PI controllers for the two tank system.

The experiments will be carried out in the middle of the tank, where the system has been identified, so the reference command will start at $0.5 \mathrm{~V}$ and will increase in $0.4 \mathrm{~V}$ for a step change (11 cm in the tank level). It will be considered as an acceptable steady-state error around $9.5 \%$ of this change resulting in $e_{s s}=0.038 \mathrm{~V}(1 \mathrm{~cm}$ in tank level).

For the RQH sampler design the hysteresis is chosen slightly greater than the measured noise $h=0.012 \mathrm{~V}(1 / 3 \mathrm{~cm})$, and by applying equation $(2.2)$ the value of the quantization level is obtained $\delta=0.064 \mathrm{~V}(1.8 \mathrm{~cm})$, resulting a $\mathrm{RQH}$ ratio $h / \delta=3 / 16$. For the SSOD the quantization value is equal to the hysteresis and to the maximum steady-state error $\delta=0.038$.

To tune the controllers for these samplers, two sets of conditions are presented in terms of classical and specific margins to the non-linearity according to the tuning procedure presented in Chapter 2. One of these sets presents low robustness margins (SLR) to the non-linearity, relying the robustness of the system exclusively on the classical robustness measures. The other set (SHR), in addition to these classical robustness measures, also requires some high robustness margins to the non-linearity.

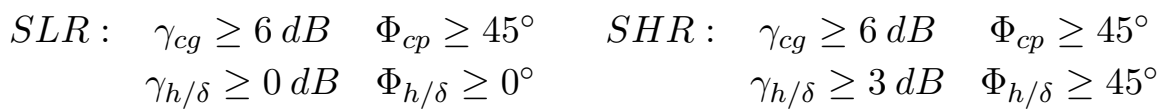

Also, according to the approach presented in Chapter 4, a controller has been tunned using the tuning table presented in Appendix C.1. As the approximated FOPTD model has a $L / \tau$ close to 0.1 , the first entry of the table has been used.

The controller's parameters using the proposed method are gathered in Table 7.1 where the parameters obtained by applying some classical tuning rules, namely Ziegler-Nichols [79] and AMIGO [4], have also been presented.

\subsubsection{DF method analysis}

Before testing these controllers on the real system a preliminary study based on the DF technique can be conducted for attempting to forecast the existence of limit cycle oscillations. The frequency response of the open-loop transfer function obtained with these controllers together with the negative inverse of the describing function of the studied RQH sampler can be seen in Figure 7.9.

According to the theoretical analysis, when a RQH sampling is applied to the loop all the controllers should avoid inducing limit cycle oscillations to the system since it does not exist 


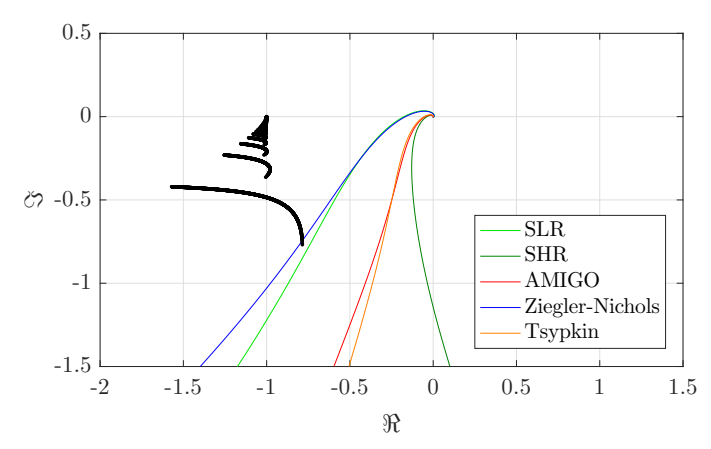

(a) SSOD

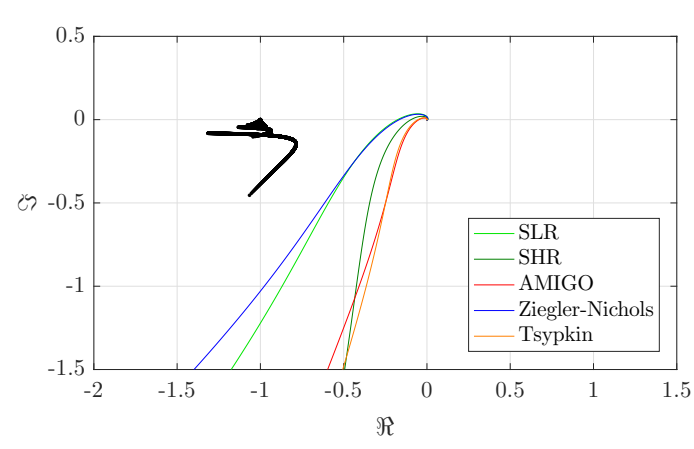

(b) RQH

Figure 7.9: Frequency response of the open-loop transfer function and the DF traces of the corresponding sampler.

intersection between the open-loop transfer function and the traces of the DF. However, in the SSOD case, both Ziegler-Nichols and SLR case present a similar frequency response, and therefore it is expected a limit cycle oscillation from them. The other methods should also avoid limit cycles.

\subsubsection{Experimental results}

The temporal responses of the system with different controllers under SSOD sampling can be seen in Figure 7.10. In these figures, the response against reference change and disturbance is presented. The reference signal is represented in black, the sampled measured signal in purple, and the temporal responses in a different color for each controller: blue for Ziegler-Nichols, red for AMIGO, light green for SLR, dark green for SHR and orange for Tsypkin.

From these figures it can be seen that Ziegler-Nichols and SLR controllers induce limit cycle oscillations in the system, which was expected since the traces of their respective open-loop transfer function intersect or are close to intersecting the DF traces. Regarding the temporal responses obtained with AMIGO controller it can be seen that it does not conduce to a limit cycle on the step change response. However, regarding the disturbance change the system enters a limit cycle. This limit cycle is not predicted by the DF technique since the oscillation type is very different from the assumed sinusoidal-like oscillations at the input of the non-linearity, therefore, further analysis is required to determine the robustness provided by this controller, which will be addressed in the next section. The proposed SHR and Tsypkin controllers avoid limit cycle oscillations as expected from the theoretical analysis.

The temporal responses obtained when replacing the SSOD by a RQH sampling are presented in Figure 7.11. From the theoretical study with the DF technique none of these controllers should induce the system to an oscillatory state. However, Ziegler-Nichols and SLR controllers 

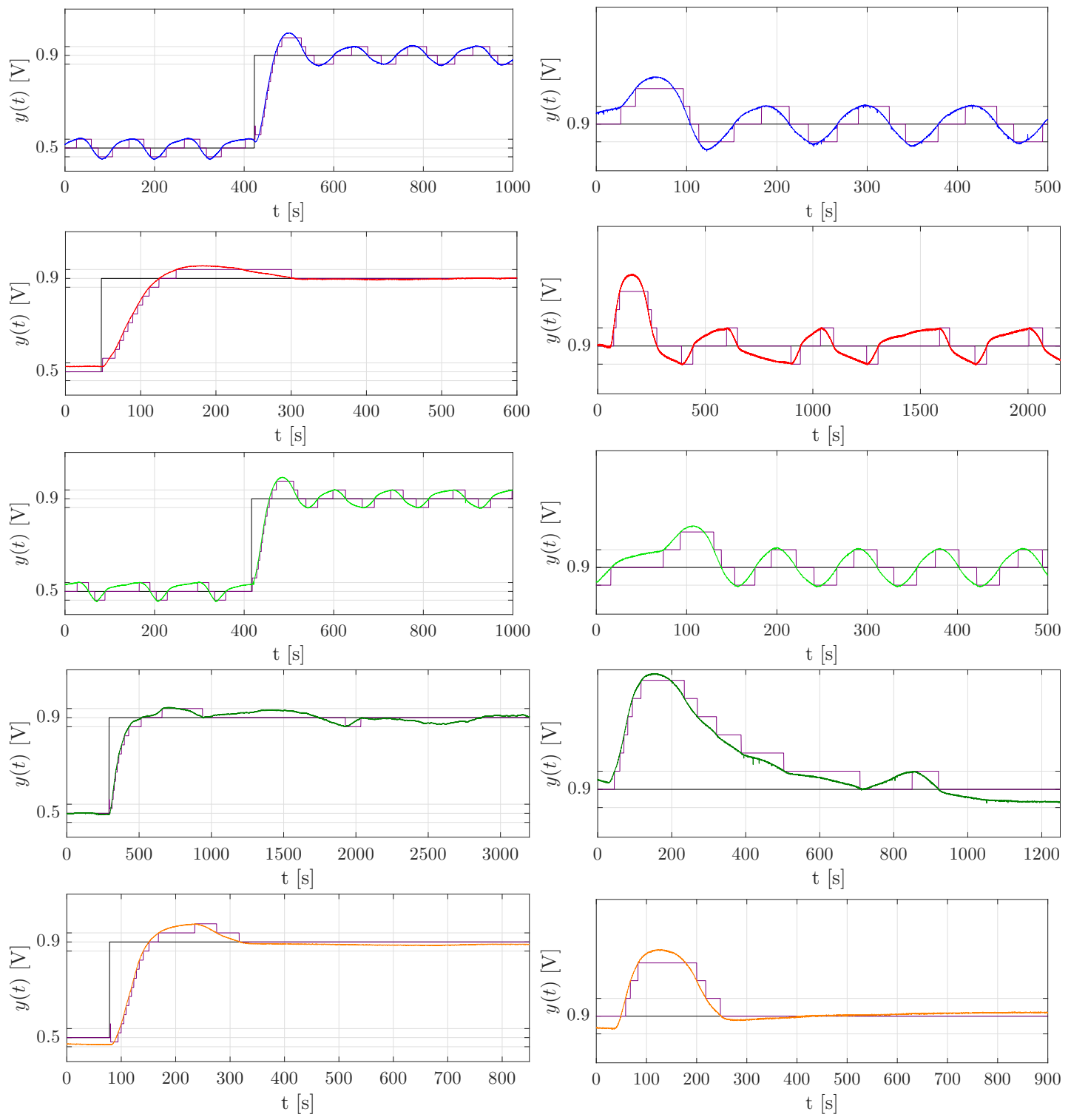

Figure 7.10: Temporal response of the system with different controllers under SSOD sampling. (Blue: Ziegler-Nichols, Red: AMIGO, Light green: SLR, Dark green: SHR, Orange: Tsypkin) 
still induce a limit cycle oscillation in their disturbance temporal response. This fact does not match the theoretical result based on the DF since their open-loop transfer function does not intersect the DF traces and they present a phase margin to the non-linearity of $\Phi_{h / \delta}=21^{\circ}$ for Ziegler-Nichols and $\Phi_{h / \delta}=24^{\circ}$ for SLR. However, it is true that the oscillations are very different from the sinusoidal input at the non-linearity that the DF technique assumes, and therefore, this kind of oscillations cannot be predicted with the DF technique. Hence, these cases will be studied with the Tsypkin Margin.

Regarding AMIGO's, SHR's and Tsypkin's step and disturbance responses it can be concluded that they do not induce limit cycle oscillations in the system response. It is true that there exist some additional switches, specially in SHR response against disturbance, but are produced as a response to variations in the system.

\subsubsection{Tsypkin's method analysis}

The precedent temporal responses present both oscillatory and non-oscillatory behaviors. Some of those behaviors were correctly predicted by the DF method, however, in those cases where hardly sinusoidal oscillations are present the DF fails to predict the apparition of a limit cycle oscillation.

Regarding the SSOD case it was expected from Ziegler-Nichols and SLR controllers to induce limit cycle oscillations in the response and from SHR and Tsypkin controllers to avoid them. In those cases the prediction provided by the DF technique is correct. This can be seen in Figure 7.12, where the open-loop transfer function for each of the described cases has been presented with their critical Tsypkin Branch (purple) which defines the robustness measure $M_{T}$. In those figures the traces of the inverse negative of the DF (black) have been also presented, which in the four cases the trace $m=1$ is very similar to the critical Tsypkin Branch (which always represents oscillations of $m=1$ type), showing the validity of the DF method in those cases. Also in this figure it can be seen how by applying the Tsypkin method the limit cycles are predicted, for Ziegler-Nichols and SLR controllers the resulting $M_{T}$ is equal to 0 which indicates the apparition of a limit cycle. On the other hand, for SHR the robustness measure is $M_{T}=0.71$ and for Tsypkin's controller $M_{T}=0.5$, measures that widely avoid the apparition of limit cycle oscillations. It is remarkable the increase of robustness from Tsypkin controller, the parameters selected from the tuning table assured a robustness $M_{T} \geq 0.2$ for the FOPTD model approximation, which is increased up to $M_{T}=0.5$ for the SOPTD model.

However, the AMIGO controller arose questions about its applicability since it largely avoided the intersection with the DF traces, but in the experimental response to disturbances it presented a behavior very similar to an oscillation where there are unattenuated harmonics, therefore, an analysis with the Tsypkin method is necessary.

As in the precedent cases studied, the open-loop transfer function have been presented in Figure 7.13 with its critical Tsypkin Branch and the obtained $M_{T}$. In the left image of the presented figure it can be noticed that the critical value of $M_{T}$ is obtained at a very low 

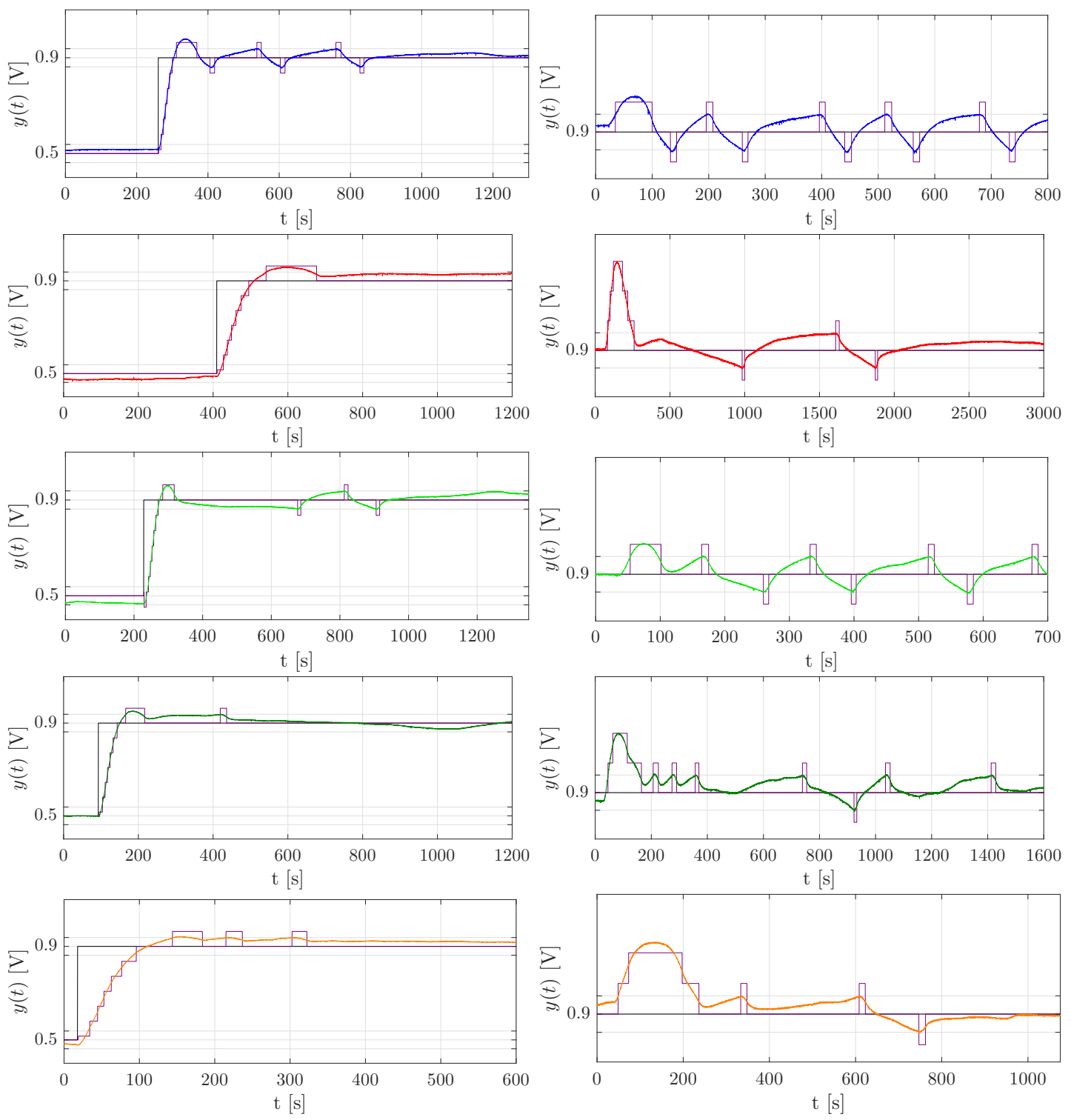

Figure 7.11: Temporal response of the system with different controllers under RQH sampling. (Blue: Ziegler-Nichols, Red: AMIGO, Light green: SLR, Dark green: SHR, Orange: Tsypkin) 

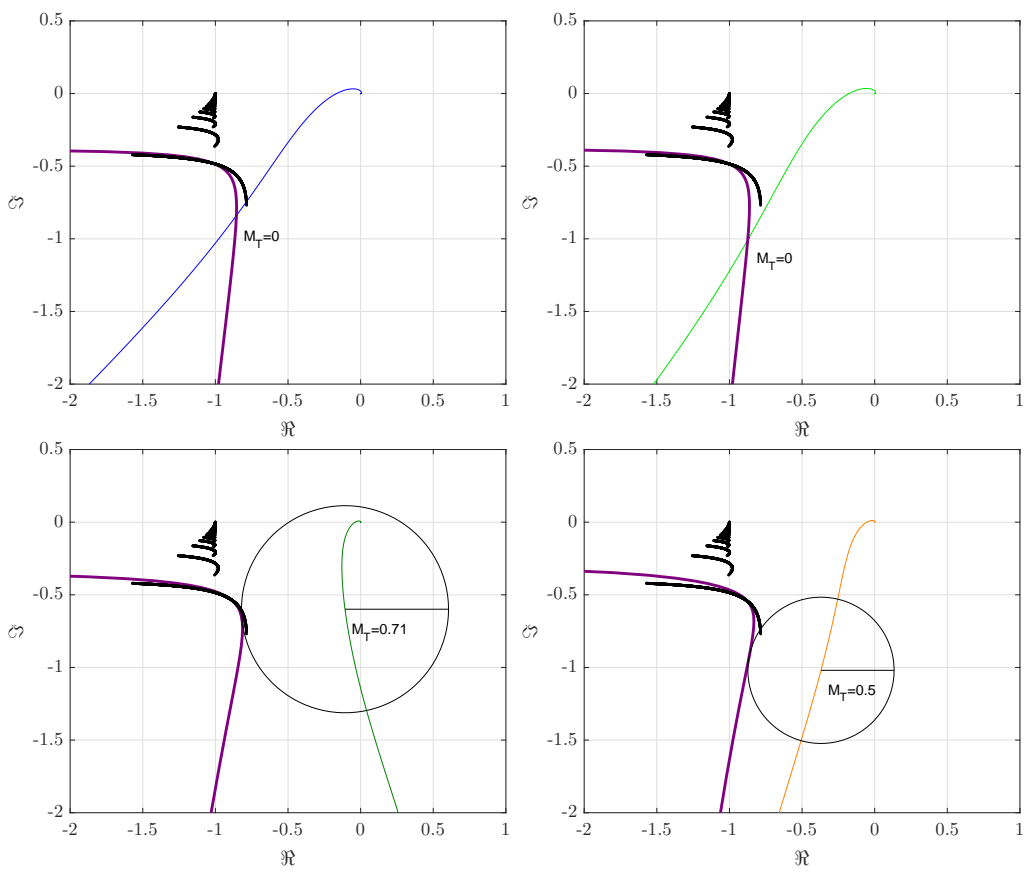

Figure 7.12: Critical Tsypkin Branch and $M_{T}$ for those cases where the DF is safely applicable. (Blue: Ziegler-Nichols, Light green: SLR, Dark green: SHR, Orange: Tsypkin)

frequency compared to where oscillations are predicted with the Describing Function. Hence, it is not surprising that the critical Tsypkin Branch does not resemble the DF traces. The value of robustness obtained $M_{T}=0.15$ should be enough to ensure that no limit cycle oscillations will be present in the loop. However, it must be pointed out that this value of $M_{T}$ has been obtained for very low frequency values with regard to the crossover frequency. This is important since the Tsypkin method requires to have well characterized the transfer function of the process under study. The identification method used in this experimental part, the step response method, provides several solutions that fit the experimental temporal data without being necessarily accurate in the frequency response. That is to say that both a FOPTD model and a second order model may provide an acceptable identification result despite being their frequency responses very different.

Therefore, since the variations that produce the uncertainties in the identification procedure (or even a possible change of model to identify with) in the frequency response, specially in the low frequencies, may be significant, it is recommended to be careful of designs that ensure robustness at low frequencies if the frequency response is not well characterized.

In this concrete example, the usage of AMIGO's controller is not advisable for the reasons aforementioned. However, with a proper frequency response identification AMIGO tuning method usually provides robust controllers.

Specially interesting is the Tsypkin analysis in the RQH case, where despite the fact that the open-loop transfer functions do not intersect the traces of the DF, some controllers still 

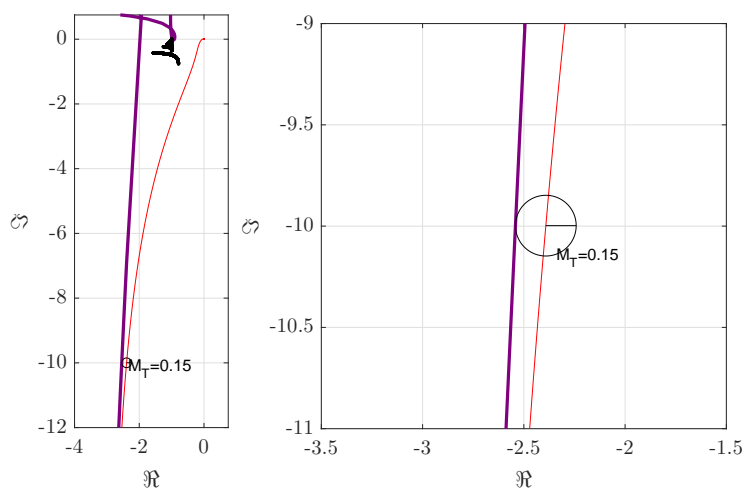

Figure 7.13: Critical Tsypkin Branch and $M_{T}$ for AMIGO controller. Whole representation and detail.

induced limit cycle oscillations in the temporal responses. Nevertheless, these oscillations are hardly sinusoidal, having the high order harmonics a remarkable influence on them.

The Tsypkin analysis has been carried out for all the controllers involved in the RQH-PI experiment and the obtained results are shown in Figure 7.14. In this figure the open-loop transfer function of each case has been represented in the Nyquist diagram with the critical Tsypkin branch in purple. In addition, the DF traces have been presented in black.

The analysis reveals that Ziegler-Nichols and SLR controllers can present limit cycle oscillations and that AMIGO, SHR and Tsypkin controllers avoid them, which corresponds with the experimental temporal responses shown in Figure 7.11.

It is also remarkable the similarities and differences between the critical Tsypkin branch and the DF traces. In those cases where unpredicted oscillations occur the DF traces differ significantly from the Tsypkin branch that leads to $M_{T}=0$, which is expected since the oscillations that appear are very different from the assumed sinusoid at the input of the non-linearity. However, in the other cases the critical Tsypkin branch corresponds and extends the trace $m=1$ of the DF approach.

\subsubsection{Control period influence}

The analysis performed in previous sections is based on all linear elements being continuous. However, the controller is discrete since it has been implemented in a BeagleBoneBlack card. This discrete implementation of the controller is predominant in any practical application and the periodicity in which the algorithms are executed could play a significant role. In previous experiments, the period of the control algorithm has been chosen very small with regard to the dynamics of the process, in that way, the analysis remains valid and its effect on the final results is negligible.

To evaluate the effect of the control period it will be considered the SSOD case with the SHR controller for which the DF approach is valid as it is shown in Figure 7.12 where it can be 


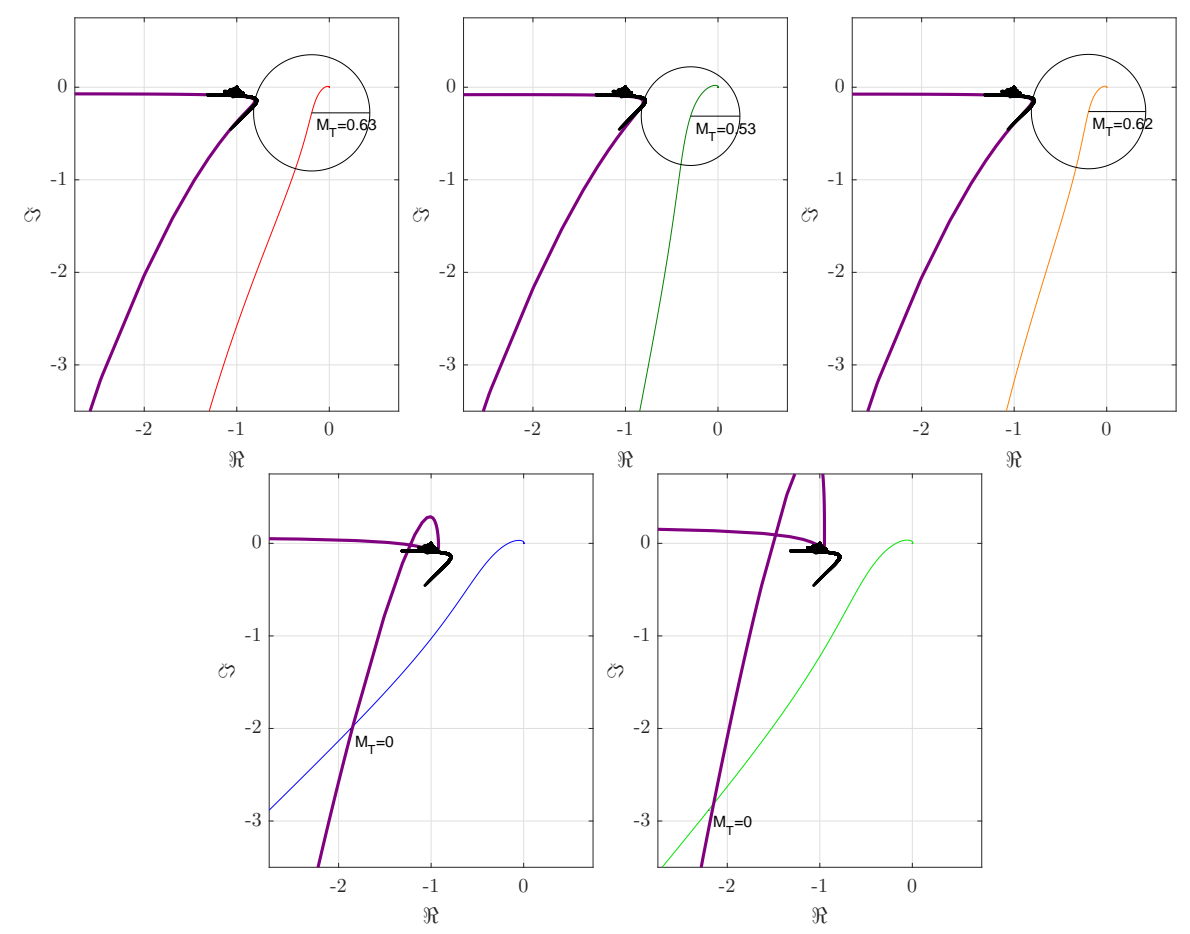

Figure 7.14: Critical Tsypkin Branch and $M_{T}$ for RQH-PI loops (Red: AMIGO, Dark green: SHR, Orange: Tsypkin, Blue: Ziegler-Nichols, Light green: SLR).

seen how the DF trace matches the critical Tsypin Branch, and has proven to have the largest robustness margin against limit cycle oscillations. One criteria to choose the control period is by evaluating the step response of the system. A common choice is to select it from 20 to 10 times smaller than the rise time $T_{s} \in\left[T_{r} / 20, T_{r} / 10\right]$. The rise time for the closed-loop step response of the theoretical process with SHR controller is $T_{r}=141 \mathrm{~s}$ and in the previous sections the control period has been $T_{s}=200 \mathrm{~ms}$.

Knowing that the implementation of the controller corresponds to a Backward Euler implementation of the integral term, the Sampled DF method has been applied to this system. The Nyquist diagram with the normalized open-loop transfer function $G_{o l}(s) / T_{s}=C^{*}(s) G(s) Z O H(s) / T_{s}$ and the convex hulls encircling the sampled DF traces for several values of $r$ (samples per oscillation period) with their respective robustness measure is presented in Figure 7.15a. In this figure, it can be seen that the convex hulls are very similar to the continuous DF traces and therefore that the impact of the control period is negligible. In addition, the robustness against limit cycle oscillations has been measured to be $D_{c h}=0.69$ for $r=978$, which is very similar to the obtained $M_{T}$ for the continuous case.

According to the rule of thumb to choose the control period, the $T_{s}$ chosen for the previous section is excessive and, since large robustness margin is available, greater control periods can be chosen. For the sake of the experiment it has been chosen two additional control periods of $T_{s}=14 \mathrm{~s}$, which correspond to the upper limit of the rule of thumb, and $T_{s}=28 \mathrm{~s}$, which falls outside the typical range. 


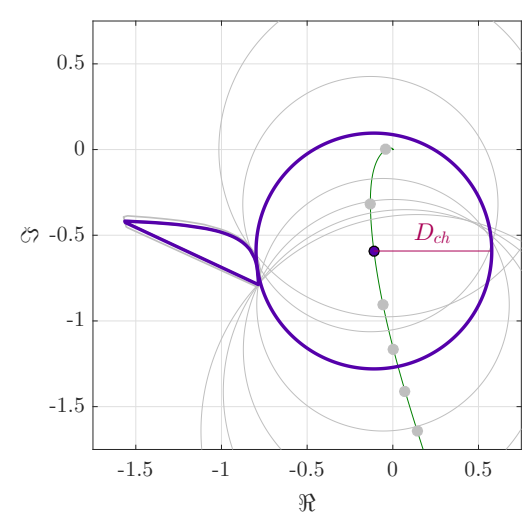

(a) $T_{s}=200 \mathrm{~ms}, D_{c h}=0.69$

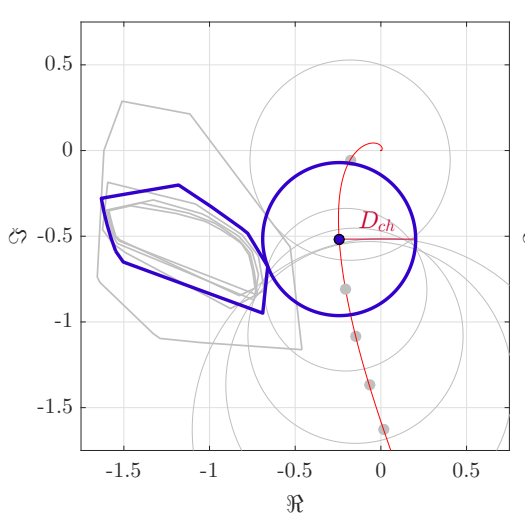

(b) $T_{s}=14 s, D_{c h}=0.45$

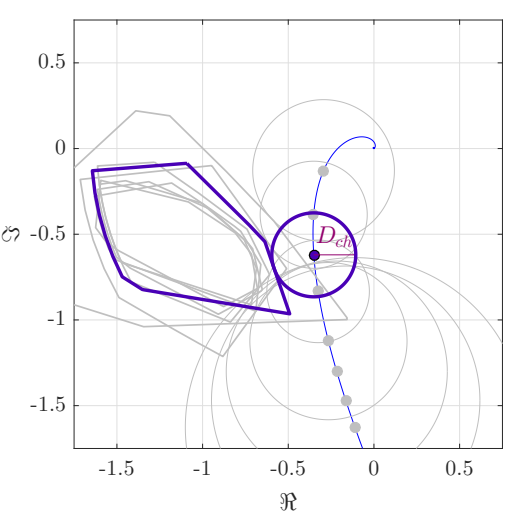

(c) $T_{s}=28 s, D_{c h}=0.25$

Figure 7.15: Sampled DF analysis for SHR controller.

As in the case with $T_{s}=200 \mathrm{~ms}$ the Sampled DF approach has been applied to the new cases. Each of the normalized open-loop transfer functions has been represented with the convex hull encircling the sampled DF traces for a range of values of $r$ in Figures $7.15 \mathrm{~b}$ and $7.15 \mathrm{c}$. As it can be seen in these figures in both cases limit cycle oscillations are avoided since $D_{c h}>0$. For the case $T_{s}=14 \mathrm{~s}$ a value $D_{c h}=0.45$ is obtained for $r=13$ and for the case $T_{s}=28 \mathrm{~s}$ a value $D_{c h}=0.25$ is obtained for $r=8$. Nevertheless, it can be observed how the robustness measure $D_{c h}$ lowers when $T_{s}$ increases, thus, increasing the control period further could result in the apparition of limit cycle oscillations.

The SHR controller has been tested on the system changing the control periods. The responses of the system against a step change in the reference input and their corresponding control action can be found in Figure 7.16. And the responses against a disturbance input in Figure 7.17 with their corresponding control action. As expected the controller avoids limit cycle oscillations with all the considered control periods.

This kind of experiments reducing the control frequency can decrease significantly the number of control action changes, however, the magnitude of these changes increases. This reduction of control action changes may be beneficial for the actuator.

\subsubsection{Summary}

In this section, a summary of the control experiences is presented. In Table 7.2 a summary of the controllers obtained with different tuning methods with the robustness that they provide is shown. The robustness parameters presented include classical phase and gain margins $\left(\Phi_{c p}, \gamma_{c g}\right)$, specific gain and phase margins to the non-linearity under study $\left(\Phi_{h / \delta}, \gamma_{h / \delta}\right)$ and the Tsypkin's robustness margin $\left(M_{T}\right)$ to the non-linearity in the loop.

The results shown in this table indicate that there exists certain correlation between classical robustness margins and the specific margins, since the higher the classical margins are, the more the specific margins increase. Nevertheless, despite this correlation, classical robustness margins 

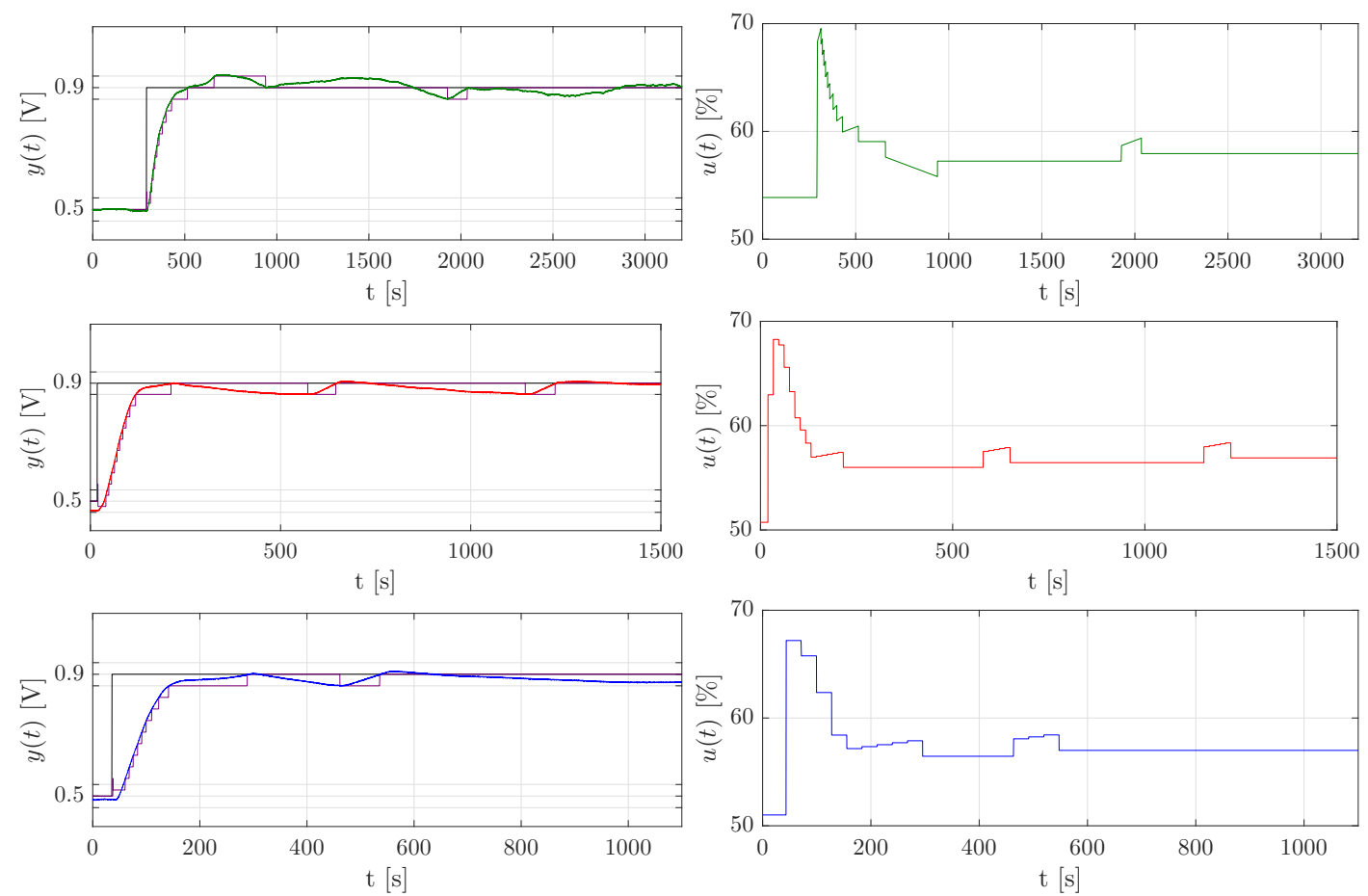

Figure 7.16: Step response of the studied system with different control periods and respective control action. $T_{s}=200 \mathrm{~ms}$ in dark green, $T_{s}=14 \mathrm{~s}$ in red and $T_{s}=28 \mathrm{~s}$ in blue.
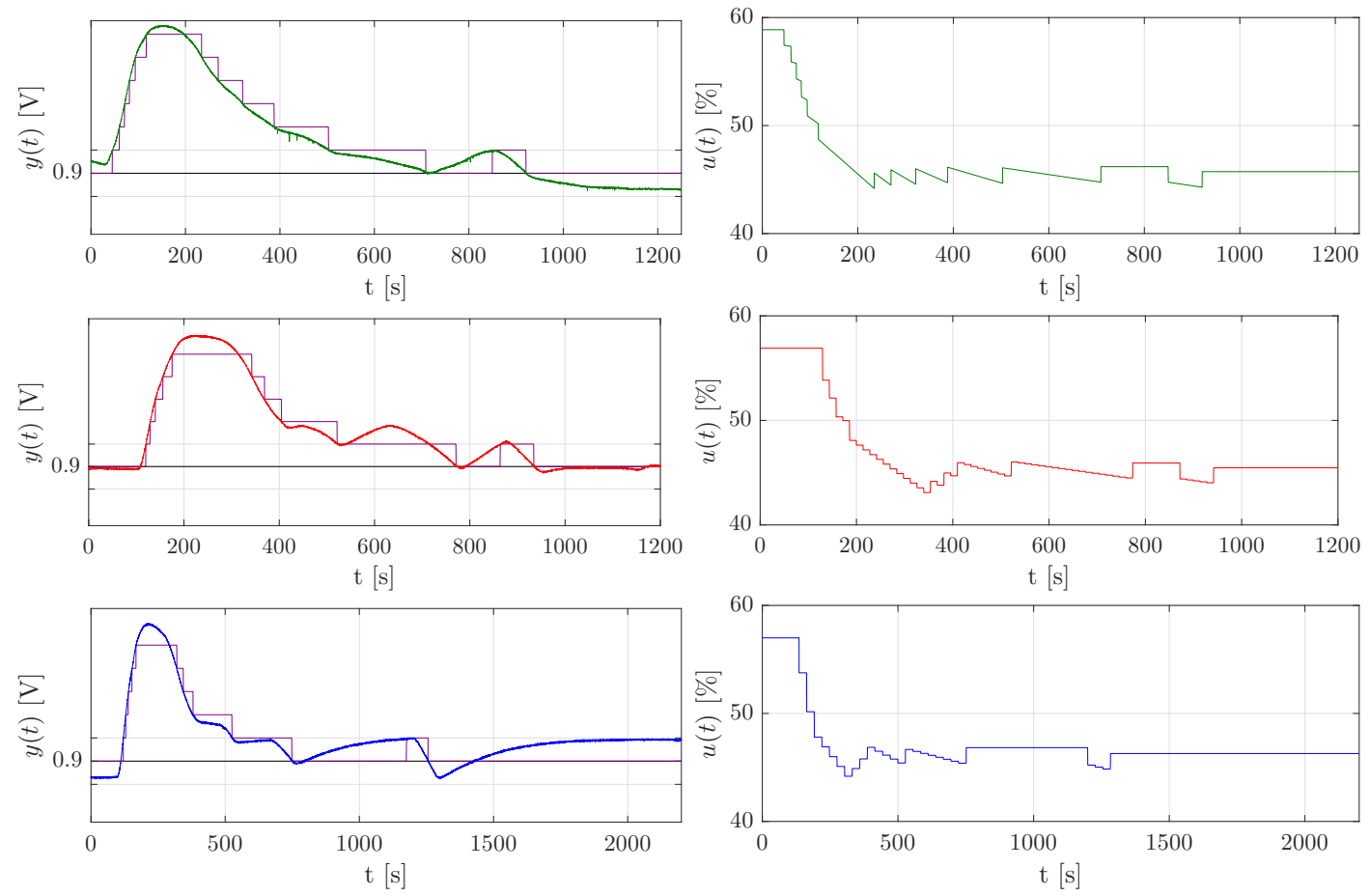

Figure 7.17: Response against disturbance of the studied system with different control periods and respective control action. $T_{s}=200 \mathrm{~ms}$ in dark green, $T_{s}=14 \mathrm{~s}$ in red and $T_{s}=28 \mathrm{~s}$ in blue. 


\begin{tabular}{c|c|c|c|c|c|c|c} 
& \multicolumn{2}{|c|}{ SLR } & \multicolumn{2}{c|}{ SHR } & \multirow{2}{*}{ AMIGO } & $\begin{array}{c}\text { Ziegler } \\
\text { Nichols }\end{array}$ & \multirow{2}{*}{ Tsypkin } \\
\cline { 2 - 5 } & SSOD & RQH & SSOD & RQH & & \\
\hline $\mathbf{K}_{\mathbf{p}}$ & 157.37 & 157.37 & 37.82 & 93.76 & 45.96 & 142.05 & 52.51 \\
\hline $\mathbf{T}_{\mathbf{i}}$ & 44.76 & 44.76 & 221.68 & 107.76 & 80.36 & 36.11 & 90.76 \\
\hline$\left.\Phi_{c p}{ }^{\circ}\right]$ & 45 & 45 & 88 & 65.7 & 67.6 & 42.5 & 69.6 \\
\hline$\gamma_{c g}[d B]$ & 15 & 15 & 28.5 & 20.4 & 26.4 & 15.6 & 25.3 \\
\hline$\Phi_{h / \delta}\left[^{\circ}\right]$ (SSOD) & 1.7 & - & 45 & - & 23 & -1.3 & 25.2 \\
\hline$\gamma_{h / \delta}[d B](\mathbf{S S O D})$ & 0.92 & - & 17.4 & - & 14.3 & -1.2 & 13.4 \\
\hline$\left.\Phi_{h / \delta}\left[^{\circ}\right] \mathbf{( R Q H}\right)$ & - & 24.1 & - & 45 & 44.8 & 21 & 47.2 \\
\hline$\left.\gamma_{h / \delta}[d B] \mathbf{R Q H}\right)$ & - & 9.4 & - & 15 & 21 & 9.7 & 20 \\
\hline$M_{T} \mathbf{( S S O D )}$ & 0 & - & 0.71 & - & 0.15 & 0 & 0.5 \\
\hline$M_{T} \mathbf{( R Q H )}$ & - & 0 & - & 0.53 & 0.63 & 0 & 0.62
\end{tabular}

Table 7.2: Summary of robustness margins provided by the controllers under study.

\begin{tabular}{|c|c|c|c|c|c|c|c|}
\hline & \multicolumn{2}{|c|}{ SLR } & \multicolumn{2}{|c|}{ SHR } & \multirow{2}{*}{ AMIGO } & \multirow{2}{*}{$\begin{array}{l}\text { Ziegler } \\
\text { Nichols }\end{array}$} & \multirow{2}{*}{ Tsypkin } \\
\hline & SSOD & RQH & SSOD & RQH & & & \\
\hline SSOD oscillation & yes & - & no & - & yes & yes & no \\
\hline SSOD DF accurate & yes & - & yes & - & no & yes & yes \\
\hline RQH oscillation & - & yes & - & no & no & yes & no \\
\hline RQH DF accurate & - & no & - & yes & yes & no & yes \\
\hline
\end{tabular}

Table 7.3: Summary of detected oscillation and validity of the DF approach.

cannot be taken as a real indicative for the behavior of the system when the type of nonlinearities under study are implemented in the control loop, since they do not determine the robustness against the limit cycle oscillations that these non-linearities induce.

As it is known, the DF technique is applicable as long as the input to the non-linear part of the loop reassembles a sinusoid and the linear part of the system filters sufficiently the output signal to consider relevant only the first harmonic, i.e. a sinusoid, that will be reintroduced at the input of the non-linear part. Therefore, the robustness margins obtained from this method will remain valid as long as this filtering hypothesis is fulfilled.

In the experimental results section, it has been showed how, despite the apparent robustness against limit cycle oscillations that the DF assured, some sustained oscillations appeared. As it has been shown lately, Tsypkin's method predicted with more accuracy all the cases. The unique conflictive case is the AMIGO controller with a SSOD sampling strategy in which, even if the robustness measure $M_{T}$ is greater than 0 , it is also true that the obtained value is found for a low frequency, and therefore, a small variation in the obtained model could alter significantly its value.

In Table 7.3, a summary of the experimental results and their relation with the analysis can be found. The table gathers whether an oscillation has been found or not for different sampling strategies and the controllers obtained with different methods. Then, an entry is included reflecting if the initial DF method used to analyze them has been accurate. It is 
considered as accurate the cases where the trace of the inverse negative of the DF for $m=1$ reassembles the critical Tsypkin Branch.

With regard to the control period refreshment experiments, the sampled DF has proven its validity since for the studied cases no limit cycles have appeared as it was expected since $D_{c h}>0$ was obtained for all the cases. In addition, for the case that can be considered as continuous, the obtained $D_{c h}$ is very similar to the Tsypkin Margin, and both are measured from a similar frequency. This fact shows the convergence of the Sampled DF to the continuous case when the control frequency is high enough to be neglected with regard to the system dynamics.

\subsection{Conclusions}

In this chapter, the implementation of applications in IEC 61499 involving the control of continuous processes has been treated. Concretely, an application in which a networked event-based control is applied has been developed. The standard facilitates the implementation of distributed applications thanks to the possibility of designing in a centralized fashion a distributed application, the ease of implementing the communication between devices and the event ruled execution of the standard.

To create the control application some BeagleBoneBlack specific function blocks have been added to the application, a specific FB has been created to implement the effect of the RQH and SSOD quantization and the rest of FB belong either to the base 4DIAC distribution or to FBDK.

An experimental framework has been prepared consisting in the control of the volume in a tank. In this setup, the actuator unit and the sensor unit are implemented in different cards which are connected via an Ethernet connection, implementing, therefore, a networked control system.

The contents treated theoretically in Chapters 2, 3, 4 and 6 have been treated experimentally. To that end, several controllers have been tunned for the system under study, and the theoretical content of previous chapters has been applied to determine the robustness or instability of each of them.

All in all, with this chapter distributed EBC has been validated experimentally as a successful control strategy, supported by a solid theoretical background. 



\section{Summary and future research}

\subsection{Summary}

This thesis addresses the robustness issues arisen from the application of EBC to a networked control system. The influence of the sampling strategy to generate the events is shown to be of paramount importance to obtain robust responses of the system. The focus has been set on sampling strategies based on the quantization of the error signal with fixed thresholds.

In this thesis, several methods to quantify the robustness of such systems are developed. The analysis is based on the describing function technique and on the Tsypkin method. Based on these methods, classical control theory can be applied to the study of the robustness of such systems, extending concepts as the gain and phase margins to the EBC paradigm. From the performed analysis, two tuning methods for PI and PID controllers are proposed, which ensure a robust behavior against the limit cycle oscillations induced by the sampler.

The issues derived from the real implementation of a EBC in a networked control system are also treated. The effect of a discrete implementation of the controller is evaluated and its robustness characterized. The theoretical analysis and tuning methods are validated with a real system.

\section{Study of new sampling schemes}

Sampling techniques based on the quantization of the error signal can be found among the most used for EBC. A popular choice among these sampling strategies is the SOD technique, which sends the sampled new data when it surpasses a certain threshold $\delta$. Despite its simplicity, it entails a problem to evaluate its robustness against limit cycle oscillation, which is that its output is not a quantization of the input, therefore, it makes the non-linearity extremely dependent on the sampling period.

To overcome this difficulty a variation of SOD, called SSOD, was introduced fixing the output and input thresholds to multiples of a quantification constant $\delta$. A frequency domain analysis was then possible thanks to well known classical control analysis techniques. 
However, it was observed that the hysteresis that SSOD introduced was sometimes excessive with regard to the noise on the signal to quantify, suggesting that its value may be reduced. In addition, in order to obtain stable responses the controllers were forced to be very slow due to the detrimental effect of the hysteresis.

Therefore, a new sampling scheme in which the quantization output and the hysteresis are decoupled has been proposed. The technique has been called RQH which stands for regular quantification with hysteresis, and it is detailed in Chapter 2. This technique reduces the hysteresis with regard to SSOD sampling while maintaining the admissible steady-state error of SSOD.

This new technique implies that the controllers can be faster since the robustness requirements to avoid limit cycle oscillations are lower with regard to SSOD. In addition, the number of generated events has been observed to be lower while still avoiding bursts of events generated by the noise.

In addition to RQH, a generalized Fixed Threshold Sampler (FTS) has been proposed in Chapter 5. This FTS includes the behavior of other well known sampling strategies by correctly choosing the appropriate parameters that define it. An analysis methodology for loops containing an FTS has been proposed. Using this analysis technique stable responses can be obtained in FTS- $C(s)$ loops if desired, besides, a relay-based control can also be deployed since the method delves on the conditions to obtain limit cycle oscillations.

\section{Development of specific analysis and tuning methods}

A lot of effort has been invested in developing sampling schemes and controllers for EBC. However, not so much has been spent in characterizing the robustness issues that these novelties entail. The studied control loop corresponds to a Wiener-Hammerstein configuration, to which classical control analysis techniques can be applied. A well known technique for such systems is the Describing Function (DF) technique, which can be applied to these loops considering that all the linear elements can be grouped and all the non-linear elements can also be grouped. Therefore, a frequency analysis of the system is possible.

Using the DF technique, the effect on the robustness of RQH sampling technique has been evaluated. From the study of the inverse negative of the DF on the Nyquist diagram, some robustness measures have been proposed in terms of gain and phase margins to the apparition of limit cycle oscillations. These measures are presented in Chapter 2, and have been used to evaluate the robustness attained by some classical tuning rules usually applied to EBC control. The results show that some controllers do not provide enough robustness to avoid limit cycle oscillations and some other tuning rules provide controllers with too high margins and, thus, slow responses, which indicates that faster, and still robust, controllers can be obtained.

Therefore, a tuning procedure for RQH-PI loops has been developed in Chapter 3. The proposed tuning procedure takes into account classical and specific robustness measures to the non-linear effect of the RQH sampling obtaining, through the minimization of the integral error, robust and fast controllers for this kind of loops. 
Nevertheless, it is known that one of the main pitfalls for the applicability of DF technique analysis is to fulfill the filtering hypothesis, which states that the linear part should attenuate the high order harmonics so that the input to the non-linear part can be considered as a sinusoid. Therefore, to analyze the effect on the robustness of the loop with a PID controller, which do not attenuate the high order harmonics, the DF technique can be at least questionable.

Hence, an exact method should be used to study this kind of loops. In Chapter 4 the Tsypkin method, which is an exact analysis tool, is used to study the robustness of SSOD-PID loops. A robustness measure called Tsypkin margin has been obtained, which exactly indicates the robustness against limit cycle oscillations induced by SSOD. The measure has been also proved effective to avoid any type of multi-leveled oscillations induced by the sampler. In addition, using this measure as foundation stone, a tuning method for PID controllers has been developed considering FOPTD models. Approximating any system by a FOPTD, tuning the controller for this FOPTD model and then applying the controller to the original system has proved to offer more robustness than the initially aimed with the FOPTD model. A Java application has been provided to easily apply this method. The Tsypkin method has been extended to also consider RQH sampling, determining the robustness regardless of the linear part filtering capabilities.

As aforementioned, a methodology to analyze FTS, based on the Dual Input Describing Function (DIDF), has been proposed in Chapter 5. This methodology not only studies the sustainability of unattenuated oscillations, but it also provides the conditions necessary to maintain a bias through the loop, being both closely related to the signals exciting the loop.

A theoretical analysis about the robustness issues arisen from the implementation of controllers in processor based devices has been presented in Chapter 6. To analyze the robustness the Sampled DF technique has been used in SSOD-PI $(z)$ loops. The obtained Sampled DF has been analyzed and it has been shown that by increasing the sampling frequency, the traces tend to the continuous DF. Also the shape of the Sampled DF reveals the existence of multiple oscillation modes within the same DF trace. A robustness measure against the apparition of limit cycle oscillations induced by the sampling strategy and the discrete implementation of the controller, called $D_{c h}$, has been developed, and several classical tuning methods have been evaluated with this measure.

\section{Implementation of Event-Based PID controllers in standard IEC 61499}

With the theoretical analysis of networked EBC loops considering different samplers and taking into account the effects on the robustness derived from the discrete implementation of the controller, in Chapter 7 the theoretical analysis and tuning methods are applied to a real control system.

The distributed control application has been developed in standard IEC 61499, which facilitates the hitherto implementation of distributed control systems provided by standard IEC 61131. IEC 61499 standard is based on the concept of Function Block (FB) to implement the functionality together with the event concept, which regulates the execution of an application, which is completely defined by the FB instances and their interconnection. 
The standard base library does not contain by default some specific features of the implementation, as data input acquisition FBs, and also the creation of some specific blocks, like the RQH quantization, is necessary. These FBs have been implemented and compiled into the runtime, which supports the deployment of the distributed application in the devices involved.

To prove the validity of the theoretical content explained in previous chapters, several controllers with different degrees of robustness have been calculated. These controllers have been tested in a real system, proving that the contents developed in this thesis are valid to analyze networked EBC systems.

\subsection{Future research}

The contributions presented with this thesis set some important base for the study of networked control and EBC analysis. However, there are still some open issues or research interests that can be further developed.

This thesis devotes its content to present and analyze Fixed Threshold Samplers, starting from a continuous approach and moving towards a discrete implementation. However, other possibilities are available, for example considering samplers based on the quantization of the integral of the error. In the same line, including more intelligence on the sensor unit could result in a beneficial decrease of the samples to be taken, for example including some prediction on the error based on the derivative of the error signal.

Regarding the tuning methods presented in this thesis, the tuning method proposed for SSOD has not been evaluated specifically for RQH samplers. Since the studies carried out with the DF technique indicate that the robustness requirements to avoid oscillations decrease with the hysteresis of the sampler, maybe evaluating the robustness on loops with RQH sampler with the Tsypkin method could derive on similar results. In that case, a tuning methodology for RQH-PID loops based on the Tsypkin margin could be developed, providing faster responses and exact robustness margins than with the current tuning methods.

In addition, the sampling period has been proved to play a paramount role on the robustness of the loop in discrete implementations of the controller. Despite this fact, this parameter has only been included as a design parameter, and not as a design result, which could lead to an optimized response with a determined sampling period without relying on a rule of thumb to determine it.

Besides, fractional order PID is a consolidated field of research with a growing acceptance by the practitioner, and the Describing Function technique has already been applied to its study. However, the effect of such controllers on the presented EBC systems has not been evaluated yet, which could lead to promising results since they are a generalization of the conventional PID with more degrees of freedom in their frequency response due to the fractional-order differential and integral operators. 


\section{Temporal response induced by a multileveled sampler}

Consider that the sampled error signal $\bar{e}(t)$ follows a ladder-type form as shown in Figure 4.5, then the expression of $\bar{e}(t)$ can be obtained through Fourier series expansion:

$$
\bar{e}(t)=\frac{a_{0}}{2}+\sum_{n=1}^{\infty}\left(a_{n} \cos \left(\omega_{n} t\right)+b_{n} \sin \left(\omega_{n} t\right)\right) .
$$

As the error signal is supposed centered around 0 and symmetric the coefficient $a_{0}$ is equal to 0 . For the other coefficients:

$$
\begin{aligned}
a_{n}= & \frac{2}{T_{o}} \int_{\rho_{1} \frac{T_{o}}{2}}^{\rho_{2} \frac{T_{o}}{2}} \delta \cos \left(\omega_{n} t\right) d t+\frac{2}{T_{o}} \int_{\rho_{2} \frac{T_{o}}{2}}^{\rho_{3} \frac{T_{o}}{2}} 2 \delta \cos \left(\omega_{n} t\right) d t+\ldots+\frac{2}{T_{o}} \int_{\rho_{m} \frac{T_{o}}{2}}^{\rho_{m+1} \frac{T_{o}}{2}} m \delta \cos \left(\omega_{n} t\right) d t+ \\
& +\frac{2}{T_{o}} \int_{\rho_{m+1} \frac{T_{o}}{2}}^{\rho_{m+2} \frac{T_{o}}{2}}(m-1) \delta \cos \left(\omega_{n} t\right) d t+\ldots+\frac{2}{T_{0}} \int_{\rho_{2 m-1} \frac{T_{0}}{2}}^{\rho_{2 m} \frac{T_{o}}{2}} \delta \cos \left(\omega_{n} t\right) d t+ \\
& +\frac{2}{T_{o}} \int_{\frac{T_{o}}{2}+\rho_{1} \frac{T_{o}}{2}}^{\frac{T_{o}}{2}+\rho_{2} \frac{T_{o}}{2}}-\delta \cos \left(\omega_{n} t\right) d t+\ldots+\frac{2}{T_{o}} \int_{\frac{T_{o}}{2}+\rho_{m} \frac{T_{o}}{2}}^{\frac{T_{o}}{2}+\rho_{m+1} \frac{T_{o}}{2}}-m \delta \cos \left(\omega_{n} t\right) d t+ \\
& +\frac{2}{T_{o}} \int_{\frac{T_{o}}{2}+\rho_{m+2} \frac{T_{o}}{2}}^{\frac{T_{o}}{2}+\rho_{m+1} \frac{T_{o}}{2}}-(m-1) \delta \cos \left(\omega_{n} t\right) d t+\ldots+\frac{2}{T_{o}} \int_{\frac{T_{o}}{2}+\rho_{2 m} \frac{T_{o}}{2}}^{-}-\delta \cos \left(\omega_{n} t\right) d t
\end{aligned}
$$


Solving and arranging the equation, the first Fourier coefficient is obtained:

$$
a_{n}=\left\{\begin{array}{cl}
0 & \text { if } \mathrm{n} \text { is even } \\
\frac{2 \delta}{n \pi}\left(\sum_{i=1}^{m} i\left(\sin \left(n \pi \rho_{i+1}\right)-\sin \left(n \pi \rho_{i}\right)\right)+\sum_{i=m+1}^{2 m-1}(2 m-i)\left(\sin \left(n \pi \rho_{i+1}\right)-\sin \left(n \pi \rho_{i}\right)\right)\right) & \text { if } n \text { is odd }
\end{array}\right.
$$

In a similar way, the second coefficient is obtained:

$$
b_{n}=\left\{\begin{array}{cl}
0 & \text { if } n \text { is even } \\
\frac{2 \delta}{n \pi}\left(\sum_{i=1}^{m} i\left(\cos \left(n \pi \rho_{i}\right)-\cos \left(n \pi \rho_{i+1}\right)\right)+\sum_{i=m+1}^{2 m-1}(2 m-i)\left(\cos \left(n \pi \rho_{i}\right)-\cos \left(n \pi \rho_{i+1}\right)\right)\right) & \text { if } n \text { is odd }
\end{array}\right.
$$

Substituting $a_{0}, a_{n}$ and $b_{n}$ in equation (A.1), $\bar{e}(t)$ is obtained. Taking into account that $e=-L \bar{e}$, and knowing that $L$ is the open-loop transfer function of the system $\left(L=G_{o l}(j \omega)\right)$, the general expression for $e(t)$ results in:

$$
\begin{aligned}
e\left(\rho_{p} \frac{T_{o}}{2}\right)=-\frac{4 \delta}{\pi} \sum_{n_{\text {odd }}}^{\infty} \frac{1}{n}\left(\Re \{ G _ { \text { ol } } ( j n \omega _ { o } ) \} \left(\sum_{i=1}^{m} i \sin \left(\frac{n \pi}{2}\left(\rho_{i+1}-\rho_{i}\right)\right) \cos \left(n \pi\left(\frac{\rho_{i+1}+\rho_{i}}{2}-\rho_{p}\right)\right)\right.\right. \\
\left.\left.+\sum_{i=m+1}^{2 m-1}(2 m-i) \sin \left(\frac{n \pi}{2}\left(\rho_{i+1}-\rho_{i}\right)\right) \cos \left(n \pi\left(\frac{\rho_{i+1}+\rho_{i}}{2}-\rho_{p}\right)\right)\right)\right) \\
-\frac{4 \delta}{\pi} \sum_{n_{\text {odd }}}^{\infty} \frac{1}{n}\left(\Im \{ G _ { \text { ol } } ( j n \omega _ { o } ) \} \left(\sum_{i=1}^{m} i \sin \left(\frac{n \pi}{2}\left(\rho_{i+1}-\rho_{i}\right)\right) \sin \left(n \pi\left(\frac{\rho_{i+1}+\rho_{i}}{2}-\rho_{p}\right)\right)\right.\right. \\
\left.\left.+\sum_{i=m+1}^{2 m-1}(2 m-i) \sin \left(\frac{n \pi}{2}\left(\rho_{i+1}-\rho_{i}\right)\right) \sin \left(n \pi\left(\frac{\rho_{i+1}+\rho_{i}}{2}-\rho_{p}\right)\right)\right)\right)
\end{aligned}
$$




\section{Appendix B}

\section{DF related calculations}

\section{B.1 RQH calculation}

The output equation of the proposed sampler, whose input-output relation is presented in Figure 2.3, is:

$$
\bar{e}(t)= \begin{cases}(i+1) \delta & \text { if } e(t) \geq\left(i+\frac{1}{2}+\frac{h}{2 \delta}\right) \delta \text { and } \bar{e}\left(t^{-}\right)=i \delta, i \in \mathbb{Z} \\ (i-1) \delta & \text { if } e(t) \leq\left(i-\frac{1}{2}-\frac{h}{2 \delta}\right) \delta \text { and } \bar{e}\left(t^{-}\right)=i \delta \\ i \delta & \text { if } e(t) \in\left[\left(i-\frac{1}{2}-\frac{h}{2 \delta}\right) \delta,\left(i+\frac{1}{2}+\frac{h}{2 \delta}\right) \delta\right] \text { and } \bar{e}\left(t^{-}\right)=i \delta\end{cases}
$$

For a sinusoidal input $e(\phi)=A \sin (\phi)$, the output of the sampler can be expressed as:

$$
\begin{aligned}
\bar{e}(\phi) & =\delta \sum_{k=1}^{i} \operatorname{sgn}\left(\left.\frac{d e(\phi)}{d \phi}\right|_{\phi_{k}}\right) \quad \forall \phi ; \phi_{i}<\phi<\phi_{i+1} \\
& =\delta \sum_{k=1}^{i} \operatorname{sgn}\left(\cos \left(\phi_{k}\right)\right) .
\end{aligned}
$$

The EG-ZOH sampler is an odd non-linearity where the past values of the input determine the value of the output in the multiple-valued regions. The Describing Function for this kind of non-linearity is calculated as:

$$
\begin{aligned}
\mathcal{N}(A, h) & =\frac{2 j}{\pi A} \int_{0}^{\pi} \bar{e}(\phi) e^{-j \phi} d \phi \\
& =\frac{2 j}{\pi A}\left(\int_{\phi_{1}}^{\phi_{2}} \delta \operatorname{sgn}\left(\cos \phi_{1}\right) e^{-j \phi} d \phi+\int_{\phi_{2}}^{\phi_{3}} \delta\left(\operatorname{sgn}\left(\cos \phi_{1}\right)+\right.\right. \\
& \left.\left.\operatorname{sgn}\left(\cos \phi_{2}\right)\right) e^{-j \phi} d \phi+\cdots+\int_{\phi_{n}}^{\pi} \delta \sum_{k=1}^{n}\left(\operatorname{sgn}\left(\cos \phi_{k}\right)\right) e^{-j \phi} d \phi\right)
\end{aligned}
$$


which can be rewritten as:

$$
\begin{aligned}
\mathcal{N}(A, h)= & \frac{2 \delta j}{\pi A}\left(\int_{\phi_{1}}^{\pi} \operatorname{sgn}\left(\cos \phi_{1}\right) e^{-j \phi} d \phi+\int_{\phi_{2}}^{\pi} \operatorname{sgn}\left(\cos \phi_{2}\right) e^{-j \phi} d \phi+\right. \\
& \left.\cdots+\int_{\phi_{n}}^{\pi} \operatorname{sgn}\left(\cos \phi_{n}\right) e^{-j \phi} d \phi\right) \\
= & \frac{2 \delta j}{\pi A} \sum_{k=1}^{n}\left(\operatorname{sgn}\left(\cos \phi_{k}\right) \int_{\phi_{k}}^{\pi} e^{-j \phi} d \phi\right) .
\end{aligned}
$$

Taking into account that:

$$
\int_{\phi_{k}}^{\pi} e^{-j \phi} d \phi=-\sin \phi_{k}-j\left(1+\cos \phi_{k}\right),
$$

equation (B.3) results in:

$$
\mathcal{N}(A, h)=\frac{2 \delta}{\pi A}\left(\sum_{k=1}^{n}\left(1+\cos \phi_{k}\right) \operatorname{sgn}\left(\cos \phi_{k}\right)-j \sum_{k=1}^{n} \sin \phi_{k} \operatorname{sgn}\left(\cos \phi_{k}\right)\right),
$$

which can be transformed to:

$$
\mathcal{N}(A, h)=\frac{2 \delta}{\pi A}\left(\sum_{k=1}^{m} \cos \phi_{k}-\sum_{k=m+1}^{2 m} \cos \phi_{k}-j\left(\sum_{k=1}^{m} \sin \phi_{k}-\sum_{k=m+1}^{2 m} \sin \phi_{k}\right)\right) .
$$

As the expressions of the $\sin \phi_{k}$ and $\cos \phi_{k}$ are known to be:

$$
\begin{gathered}
\sin \phi_{k}= \begin{cases}\frac{\delta}{A}\left(k-\frac{1}{2}+\frac{h}{2 \delta}\right) & \text { if } k=1,2, \ldots, m \\
\frac{\delta}{A}\left(2 m-k+\frac{1}{2}-\frac{h}{2 \delta}\right) & \text { if } k=m+1, m+2, \ldots, 2 m\end{cases} \\
\cos \phi_{k}= \begin{cases}+\sqrt{1-\left(\frac{\delta}{A}\left(k-\frac{1}{2}+\frac{h}{2 \delta}\right)\right)^{2}} & \text { if } k=1,2, \ldots, m \\
-\sqrt{1-\left(\frac{\delta}{A}\left(2 m-k+\frac{1}{2}-\frac{h}{2 \delta}\right)\right)^{2}} & \text { if } k=m+1, m+2, \ldots, 2 m\end{cases}
\end{gathered}
$$

can be introduced in equation (B.6) which results in:

$$
\begin{gathered}
\mathcal{N}(A, h)=\frac{2 \delta}{A \pi}\left[\sum_{k=1}^{m} \sqrt{1-\left(\frac{\delta}{A}\left(k+\frac{h}{2 \delta}-\frac{1}{2}\right)\right)^{2}}+\right. \\
\left.\sum_{k=m+1}^{2 m} \sqrt{1-\left(\frac{\delta}{A}\left(2 m-k-\frac{h}{2 \delta}+\frac{1}{2}\right)\right)^{2}}\right]-j \frac{2 h m \delta}{A^{2} \pi}
\end{gathered}
$$




\section{B.2 DIDF Calculation}

The input-output relationship of a FTS, whose main parameters are represented in Figure 5.2, is given by:

$$
\bar{x}(t)=\left\{\begin{array}{lll}
\Delta \epsilon-\frac{\epsilon}{2}+\epsilon(i+1) & \text { if } & x(t) \geq \Delta \delta+\frac{h}{2}+\delta i \quad \text { and } \quad \bar{x}\left(t^{-}\right)=\Delta \epsilon-\frac{\epsilon}{2}+\epsilon i, \quad i \in \mathbb{Z} \\
\Delta \epsilon-\frac{\epsilon}{2}+\epsilon(i-1) & \text { if } & x(t) \leq \Delta \delta-\frac{h}{2}+\delta(i-1) \quad \text { and } \quad \bar{x}\left(t^{-}\right)=\Delta \epsilon-\frac{\epsilon}{2}+\epsilon i \\
\Delta \epsilon-\frac{\epsilon}{2}+\epsilon i & \text { if } & x(t) \in\left[\Delta \delta-\frac{h}{2}+\delta(i-1), \Delta \delta+\frac{h}{2}+\delta i\right] \quad \text { and } \quad \bar{x}\left(t^{-}\right)=\Delta \epsilon-\frac{\epsilon}{2}+\epsilon i
\end{array}\right.
$$

Let the input to the FTS be described by $x(\phi)=A \sin (\phi)+B$. Then, the output can be expressed as:

$$
\begin{aligned}
\bar{x}(\phi) & =\Delta \epsilon-\frac{\epsilon}{2}+\epsilon i_{0}+\epsilon \sum_{k=1}^{i} \operatorname{sgn}\left(\left.\frac{d x(\phi)}{d \phi}\right|_{\phi_{k}}\right) \quad \forall \phi ; \phi_{i}<\phi<\phi_{i+1} \\
& =\Delta \epsilon-\frac{\epsilon}{2}+\epsilon i_{0}+\epsilon \sum_{k=1}^{i} \operatorname{sgn}\left(\cos \left(\phi_{k}\right)\right),
\end{aligned}
$$

where $i_{0}$ is the level around which the oscillation is centered. Under this assumption of the form of $x(\phi)$ and because of a possible output bias that propitiates this type of oscillation, the Dual Input Describing Function (DIDF) must be used. This variant of the Describing Function has two components, one related to the sustainability of the bias, and another one related to the dynamic part of the signal, which can be respectively calculated with the following expressions:

$$
\mathcal{N}_{B}=\frac{1}{2 \pi B} \int_{0}^{2 \pi} \bar{x}(\phi) d \phi
$$

and

$$
\mathcal{N}_{A}=\frac{j}{\pi A} \int_{0}^{2 \pi} \bar{x}(\phi) e^{-j \phi} d \phi .
$$

Developing, for example, for $\mathcal{N}_{A}$ :

$$
\begin{array}{r}
\mathcal{N}_{A}=\frac{j}{\pi A}\left[\int_{0}^{\phi_{1}}\left(\Delta \epsilon-\frac{\epsilon}{2}+\epsilon i_{0}\right) e^{-j \phi} d \phi+\int_{\phi_{1}}^{\phi_{2}}\left(\Delta \epsilon-\frac{\epsilon}{2}+\epsilon i_{0}+\epsilon \operatorname{sgn}\left(\cos \left(\phi_{1}\right)\right)\right) e^{-j \phi} d \phi+\ldots\right. \\
+\int_{\phi_{2 m_{\text {sup }}+2 m_{\text {inf }}-1}}^{\phi_{2 m_{\text {sup }}+2 m_{\text {inf }}}}\left(\Delta \epsilon-\frac{\epsilon}{2}+\epsilon i_{0}+\epsilon \sum_{k=1}^{2 m_{\text {sup }}+2 m_{\text {inf }}-1} \operatorname{sgn}\left(\cos \left(\phi_{k}\right)\right)\right) e^{-j \phi} d \phi+ \\
\left.+\int_{\phi_{2 m_{\text {sup }}+2 m_{\text {inf }}}}^{2 \pi}\left(\Delta \epsilon-\frac{\epsilon}{2}+\epsilon i_{0}+\epsilon \sum_{k=1}^{2 m_{\text {sup }}+2 m_{\text {inf }}} \operatorname{sgn}\left(\cos \left(\phi_{k}\right)\right)\right) e^{-j \phi} d \phi\right]
\end{array}
$$


where $m_{\text {sup }}$ and $m_{\text {inf }}$ are the number of levels crossed in upwards and downwards direction. Arranging terms:

$$
\mathcal{N}_{A}=\frac{j}{\pi A}\left[\left(\Delta \epsilon-\frac{\epsilon}{2}+\epsilon i_{0}\right) \int_{0}^{2 \pi} e^{-j \phi} d \phi+\epsilon \sum_{k=1}^{2 m_{\text {sup }}+2 m_{\text {inf }}} \int_{\phi_{k}}^{2 \pi} \operatorname{sgn}\left(\cos \left(\phi_{k}\right)\right) e^{-j \phi} d \phi\right] .
$$

The first integral equals 0 , and for the second, it is known that:

$$
\operatorname{sgn}\left(\cos \left(\phi_{i}\right)\right)= \begin{cases}+1 & 1 \leq i \leq m_{\text {sup }} \\ -1 & m_{\text {sup }}<i \leq 2 m_{\text {sup }}+m_{\text {inf }} \\ +1 & 2 m_{\text {sup }}+m_{\text {inf }}<i \leq 2 m_{\text {sup }}+2 m_{\text {inf }}\end{cases}
$$

After some straightforward algebra calculations and arrangements the following expression is found:

$$
\begin{aligned}
\mathcal{N}_{A}= & \frac{\epsilon}{\pi A} \sum_{k=-m_{\text {inf }}}^{m_{\text {sup }}-1}\left[\sqrt{1-\left(\frac{1}{A}\left(\Delta \delta+\frac{h}{2}-B+\delta\left(k+i_{0}\right)\right)\right)^{2}}+\sqrt{1-\left(\frac{1}{A}\left(\Delta \delta-\frac{h}{2}-B+\delta\left(k+i_{0}\right)\right)\right)^{2}}\right] \\
& -j \frac{\epsilon h}{\pi A^{2}}\left[m_{\text {sup }}+m_{\text {inf }}\right] .
\end{aligned}
$$

The same procedure is followed to obtain the expression of $\mathcal{N}_{B}$ :

$$
\begin{aligned}
\mathcal{N}_{B}= & \frac{2 \Delta \epsilon+\epsilon\left(m_{\text {sup }}-m_{\text {inf }}+2 i_{0}-1\right)}{2 B} \\
& -\frac{\epsilon}{2 \pi B} \sum_{k=-m_{\text {inf }}}^{m_{\text {sup }}-1}\left[\arcsin \left(\frac{1}{A}\left(\Delta \delta+\frac{h}{2}-B+\delta\left(k+i_{0}\right)\right)\right)+\arcsin \left(\frac{1}{A}\left(\Delta \delta-\frac{h}{2}-B+\delta\left(k+i_{0}\right)\right)\right)\right] .
\end{aligned}
$$

The expressions for $m_{\text {sup }}$ and $m_{\text {inf }}$ can be calculated with:

$$
m_{\text {sup }}=\left\lfloor\frac{A+B-\Delta \delta-h / 2-\delta i_{0}}{\delta}\right\rfloor+1, \quad m_{\text {inf }}=-\left\lfloor\frac{-A+B-\Delta \delta+h / 2-\delta i_{0}}{\delta}\right\rfloor-1
$$

and the initial level $i_{0}$ with:

$$
i_{0}=\left\{\begin{array}{ll}
\left\lfloor\frac{B-\Delta \delta-h / 2}{\delta}\right\rfloor+1 & \text { if } \quad B \geq 0 \\
\left\lfloor\frac{B-\Delta \delta+h / 2}{\delta}\right\rfloor & \text { if } \quad B<0
\end{array} .\right.
$$

\section{B.3 DIDF maintenance of the bias}

Depending on the process and controller in the loop there exist different situations which define the necessary conditions to obtain a sustained bias. The disturbance and reference signals have 


\begin{tabular}{|c|c|c|c|}
\hline \multicolumn{3}{|c|}{ Form of $C(s)$ and $G(s)$} & Conditions \\
\hline \multirow{5}{*}{$C(s)=L_{1}(s) s^{n}$} & $G(s)=L_{2}(s) s^{m}$ & & $B=r(\infty)$ \\
\hline & $G(s)=L_{2}(s)$ & & $B=r(\infty)-p(\infty) L_{2}(0)$ \\
\hline & \multirow{3}{*}{$G(s)=L_{2}(s) / s^{m}$} & $n>m$ & $p(\infty)=0 \& B=r(\infty)$ \\
\hline & & $n=m$ & $p(\infty)=0 \& B\left(1+\mathcal{N}_{B} L_{1}(0) L_{2}(0)\right)=r(\infty)$ \\
\hline & & $n<m$ & $p(\infty)=0 \& B=0$ \\
\hline \multirow{3}{*}{$C(s)=L_{1}(s)$} & $G(s)=L_{2}(s) s^{m}$ & & $B=r(\infty)$ \\
\hline & $G(s)=L_{2}(s)$ & & $B\left(1+\mathcal{N}_{B} L_{1}(0) L_{2}(0)\right)=r(\infty)-p(\infty) L_{2}(0)$ \\
\hline & $G(s)=L_{2}(s) / s^{m}$ & & $B \mathcal{N}_{B}=-p(\infty) / L_{1}(0)$ \\
\hline \multirow{5}{*}{$C(s)=L_{1}(s) / s^{n}$} & $G(s)=L_{2}(s) / s^{m}$ & & $B=0$ \\
\hline & $G(s)=L_{2}(s)$ & & $B=0$ \\
\hline & \multirow{3}{*}{$G(s)=L_{2}(s) s^{m}$} & $n<m$ & $B=r(\infty)$ \\
\hline & & $n=m$ & $B\left(1+\mathcal{N}_{B} L_{1}(0) L_{2}(0)\right)=r(\infty)$ \\
\hline & & $n>m$ & $B=0$ \\
\hline
\end{tabular}

Table B.1: Expanded requirements for any combination of process and controller for the maintenance of the bias under step-like changes in reference and disturbance inputs. $L_{1}(s)$ and $L_{2}(s)$ have neither poles nor zeros at the origin and it is considered that $n, m \geq 1$.

been considered to be step-like signals of magnitude $p(\infty)$ and $r(\infty)$ respectively, resulting in the oscillation conditions to be a function of the process and controller involved. Then, the different combinations between these elements and their respective oscillation condition are presented in Table B.1.

\section{B.4 Sampled DF calculation}

The sampled describing function which relates the input and output of the non-linear element in the system can be computed in the following way:

$$
\mathcal{N}\left(\delta, A, T_{s}\right)=\frac{\text { Phasor representation of fundamental component of } \bar{e}^{*}}{\text { Phasor representation of } e} .
$$

Firstly, the phasor representation of $e$ can be easily obtained since for the DF calculations:

$$
e(t)=A \sin \left(\omega_{o} t\right)=A \cos \left(\omega_{o} t+\frac{3 \pi}{2}\right)=\Re\left\{A e^{j\left(\omega_{o} t+\frac{3 \pi}{2}\right)}\right\} .
$$

For the phasor representation of the fundamental component of $\bar{e}^{*}$, an harmonic analysis 
using Fourier series has been done. Expressing $\bar{e}^{*}(t)$ as:

$$
\bar{e}^{*}(t)=\bar{e}(t) \cdot \delta_{P T}(t)
$$

where the pulse train $\delta_{P T}$ is defined as:

$$
\delta_{P T}(t)=\sum_{k=-\infty}^{\infty} \delta_{D}\left(t-\tau-k T_{s}\right),
$$

where $\delta_{D}$ is the Dirac delta function, $T_{s}$ is the sampling period and $\tau$ the time lag between the initial zero-crossing of $e(t)$ and the first sample (which is bounded between 0 and $T_{s}$ ).

To obtain the fundamental component of $\bar{e}^{*}(t)$, firstly, the Fourier series representing $\bar{e}(t)$ is obtained:

$$
\begin{aligned}
\hat{\bar{e}}(t)= & -\frac{\delta}{j \pi} \sum_{n=-\infty}^{\infty} \frac{1}{n}\left\{\sum_{i=1}^{m} i\left[e^{-j n \omega_{o} t_{i+1}}-e^{-j n \omega_{o} t_{i}}\right]+\right. \\
& \left.\sum_{i=m+1}^{2 m-1}(2 m-i)\left[e^{-j n \omega_{o} t_{i+1}}-e^{-j n \omega_{o} t_{i}}\right]\right\} e^{j n \omega_{o} t},
\end{aligned}
$$

where $t_{n}$ are the times where level switches are produced. And secondly, the Fourier series representation of $\delta_{P T}$ is obtained:

$$
\hat{\delta}_{P T}(t)=\frac{1}{T_{s}} \sum_{k=-\infty}^{\infty} e^{j k \omega_{s}(t-\tau)} .
$$

Multiplying both:

$$
\begin{array}{r}
\hat{\bar{e}}^{*}(t)=-\frac{\delta}{j \pi T_{s}} \sum_{k=-\infty}^{\infty} \sum_{n=-\infty}^{\infty} \frac{1}{n}\left\{\sum_{i=1}^{m} i\left[e^{-j n \omega_{o} t_{i+1}}-e^{-j n \omega_{o} t_{i}}\right]+\right. \\
\left.\sum_{i=m+1}^{2 m-1}(2 m-i)\left[e^{-j n \omega_{o} t_{i+1}}-e^{-j n \omega_{o} t_{i}}\right]\right\} e^{j n \omega_{o} t} e^{j k \omega_{s}(t-\tau)} .
\end{array}
$$

To obtain the fundamental harmonic from this expression, attention has to be paid to the exponents that imply the variable $t$, which can be grouped in a single expression:

$$
j\left(n \omega_{o}+k \omega_{s}\right) t-j k \omega_{s} \tau
$$

Then, the part that multiplies $t$ has to be the fundamental frequency, i.e. either $+\omega_{o}$ or $-\omega_{o}$. Taking $r$ as the ratio between the oscillation and sampling period $\left(r=T_{o} / T_{s}\right)$, the relation between the harmonics of the sampling $(k)$ and of the signal $\bar{e}(t)(n)$ can be obtained to calculate 
the fundamental frequency of $\bar{e}^{*}(t)$ :

$$
\begin{array}{cl}
n \omega_{o}+k \omega_{s}=\omega_{o} & n \omega_{o}+k \omega_{s}=-\omega_{o} \\
n+k \frac{\omega_{s}}{\omega_{o}}=1 & n+k \frac{\omega_{s}}{\omega_{o}}=-1 \\
n=1-k r & n=-1-k r
\end{array}
$$

Thus, the relation of harmonics to consider are both $n=1-k r$ and $n=-1-k r$. The expression of the fundamental harmonic of $\bar{e}^{*}(t)$ is:

$$
\begin{array}{r}
\hat{\bar{e}}^{*}(t)=-\frac{\delta}{j \pi T_{s}} \sum_{k=-\infty}^{\infty}\left\{\frac { 1 } { 1 - r k } \left\{\sum_{i=1}^{m} i\left[e^{-j(1-r k) \omega_{o} t_{i+1}}-e^{-j(1-r k) \omega_{o} t_{i}}\right]+\right.\right. \\
\left.\sum_{i=m+1}^{2 m-1}(2 m-i)\left[e^{-j(1-r k) \omega_{o} t_{i+1}}-e^{-j(1-r k) \omega_{o} t_{i}}\right]\right\} e^{j \omega_{o} t}+ \\
\frac{1}{-1-r k}\left\{\sum_{i=1}^{m} i\left[e^{-j(-1-r k) \omega_{o} t_{i+1}}-e^{-j(-1-r k) \omega_{o} t_{i}}\right]+\right. \\
\left.\left.\sum_{i=m+1}^{2 m-1}(2 m-i)\left[e^{-j(-1-r k) \omega_{o} t_{i+1}}-e^{-j(-1-r k) \omega_{o} t_{i}}\right]\right\} e^{-j \omega_{o} t}\right\} e^{-j k \omega_{s} \tau} .
\end{array}
$$

Taking into account that:

$$
e^{-j(1-r k) \omega_{o} t_{i}}=\left(e^{j \omega_{o} t_{i}}\right)^{r k-1}=\left(\cos \left(\omega t_{i}\right)+j \sin \left(\omega_{o} t_{i}\right)\right)^{r k-1}
$$

and:

$$
\begin{aligned}
& \sin \left(\omega_{o} t_{i}\right)= \begin{cases}\frac{i \delta}{A} & \text { if } \quad i=1,2, \ldots, m \\
(2 m-i) \frac{\delta}{A} & \text { if } \quad i=m+1, m+2, \ldots, 2 m\end{cases} \\
& \cos \left(\omega_{o} t_{i}\right)= \begin{cases}+\sqrt{1-\left(\frac{i \delta}{A}\right)^{2}} & \text { if } \quad i=1,2, \ldots, m \\
-\sqrt{1-\left((2 m-i) \frac{\delta}{A}\right)^{2}} & \text { if } \quad i=m+1, m+2, \ldots, 2 m\end{cases}
\end{aligned}
$$

the expression of $\hat{\bar{e}}^{*}(t)$ can be simplified and its phasor representation obtained after some straightforward algebra calculus. Then, the ratio between the phasor of $\hat{\bar{e}}^{*}(t)$ and the phasor of 
$e(t)$ resulting in the Sampled DF:

$$
\begin{aligned}
& \mathcal{N}\left(\delta / A, r, \tau, T_{s}\right)=\frac{2 \delta}{\pi T_{s} A} \sum_{k=-\infty}^{\infty} \frac{e^{-j k 2 \pi \tau / T_{s}}}{r k-1}\left[\sum _ { i = 1 } ^ { m - 1 } \left\{\left(-\sqrt{1-\left(\frac{i \delta}{A}\right)^{2}}+j \frac{i \delta}{A}\right)^{r k-1}\right.\right. \\
& \left.\left.-\left(\sqrt{1-\left(\frac{i \delta}{A}\right)^{2}}+j \frac{i \delta}{A}\right)^{r k-1}\right\}-\left(\sqrt{1-\left(\frac{m \delta}{A}\right)^{2}}+j \frac{m \delta}{A}\right)^{r k-1}+(-1)^{r k-1}\right] .
\end{aligned}
$$




\section{Tuning methods}

\section{C.1 Tuning table for optimum SSOD}

See Table C.1.

\section{C.2 Flowchart for RQH tuning procedure}

The proposed tuning procedure can be deployed by following the flowchart in Figure C.1. 


\begin{tabular}{|c|c|c|c|c|c|c|c|c|c|c|c|c|c|c|c|}
\hline \multirow{2}{*}{$\mathbf{L} / \tau$} & \multicolumn{3}{|c|}{$\overline{\mathbf{C}}(\infty)<\mathbf{1}$} & \multicolumn{3}{|c|}{$\overline{\mathbf{C}}(\infty)<\mathbf{2}$} & \multicolumn{3}{|c|}{$\overline{\mathbf{C}}(\infty)<\mathbf{5}$} & \multicolumn{3}{|c|}{$\overline{\mathbf{C}}(\infty)<\mathbf{1 0}$} & \multicolumn{3}{|c|}{$\forall \overline{\mathbf{C}}(\infty)$} \\
\hline & $\overline{\mathbf{K}_{\mathbf{p}}}$ & $\overline{\mathbf{T}_{\mathbf{i}}}$ & $\mathbf{N}$ & $\overline{\mathbf{K}_{\mathbf{p}}}$ & $\overline{\mathbf{T}_{\mathbf{i}}}$ & $\mathbf{N}$ & $\overline{\mathbf{K}_{\mathrm{p}}}$ & $\overline{\mathbf{T}_{\mathrm{i}}}$ & $\mathbf{N}$ & $\overline{\mathbf{K}_{\mathrm{p}}}$ & $\overline{\mathbf{T}_{\mathbf{i}}}$ & $\mathbf{N}$ & $\overline{\mathbf{K}_{\mathbf{p}}}$ & $\overline{\mathbf{T}_{\mathrm{i}}}$ & $\mathrm{N}$ \\
\hline 0.1 & & & & & & & 3.97 & 7.54 & & * & $*$ & & $*$ & * & \\
\hline 0.2 & & & & & & & 2.44 & 3.22 & 0.5 & 2.7 & 3.0 & 2.0 & 2.9 & 3.13 & 20.0 \\
\hline 0.3 & & & & 1.97 & 2.97 & & 2.01 & 2.39 & 1.0 & 2.17 & 2.34 & 3.0 & 2.46 & 2.37 & 20.0 \\
\hline 0.4 & & & & 1.64 & 2.34 & & 1.66 & 1.9 & 1.0 & 1.87 & 2.0 & 4.0 & 2.07 & 2.05 & 20.0 \\
\hline 0.5 & & & & 1.41 & 2.01 & & 1.55 & 1.65 & 2.0 & 1.68 & 1.69 & 4.0 & 1.78 & 1.79 & 12.0 \\
\hline 0.6 & & & & 1.15 & 1.38 & 0.5 & 1.36 & 1.43 & 2.0 & 1.45 & 1.59 & 5.0 & 1.48 & 1.6 & 6.0 \\
\hline 0.7 & & & & 1.06 & 1.28 & 0.5 & 1.24 & 1.4 & 2.0 & 1.35 & 1.51 & 5.0 & * & $*$ & $*$ \\
\hline 0.8 & 1.0 & 1.9 & & 0.96 & 1.18 & 0.5 & 1.14 & 1.31 & 2.0 & 1.25 & 1.47 & 7.0 & 1.26 & 1.48 & 8.0 \\
\hline 0.9 & 0.94 & 1.36 & & 0.95 & 1.12 & 1.0 & 1.08 & 1.28 & 3.0 & $*$ & $*$ & $*$ & * & $*$ & $*$ \\
\hline 1.0 & 0.9 & 1.35 & & 0.91 & 1.07 & 1.0 & 0.98 & 1.2 & 2.0 & 1.05 & 1.29 & 4.0 & 1.1 & 1.37 & 12.0 \\
\hline 1.1 & 0.83 & 1.23 & & 0.85 & 1.05 & 1.0 & 0.99 & 1.22 & 4.0 & $*$ & $*$ & $*$ & $*$ & $*$ & $*$ \\
\hline 1.2 & 0.79 & 1.22 & & 0.81 & 1.0 & 1.0 & 0.89 & 1.09 & 2.0 & $*$ & $*$ & $*$ & $*$ & $*$ & $*$ \\
\hline 1.3 & 0.74 & 1.13 & & 0.77 & 0.96 & 1.0 & 0.87 & 1.1 & 3.0 & * & $*$ & $*$ & $*$ & $*$ & $*$ \\
\hline 1.4 & 0.72 & 1.13 & & 0.74 & 0.95 & 1.0 & 0.84 & 1.06 & 3.0 & $*$ & $*$ & $*$ & $*$ & $*$ & $*$ \\
\hline 1.5 & 0.65 & 0.87 & 0.5 & 0.71 & 0.91 & 1.0 & 0.83 & 1.07 & 5.0 & $*$ & $*$ & $*$ & $*$ & $*$ & $*$ \\
\hline 1.6 & 0.62 & 0.84 & 0.5 & 0.68 & 0.88 & 1.0 & 0.8 & 1.04 & 5.0 & $*$ & $*$ & $*$ & * & $*$ & $*$ \\
\hline 1.7 & 0.61 & 0.81 & 0.5 & 0.67 & 0.86 & 1.0 & 0.78 & 1.01 & 5.0 & $*$ & * & $*$ & * & $*$ & $*$ \\
\hline 1.8 & 0.6 & 0.83 & 0.5 & 0.65 & 0.83 & 1.0 & 0.71 & 0.91 & 2.0 & $*$ & $*$ & $*$ & $*$ & $*$ & $*$ \\
\hline 1.9 & 0.58 & 0.8 & 0.5 & 0.63 & 0.84 & 1.0 & 0.69 & 0.88 & 2.0 & $*$ & $*$ & $*$ & $*$ & $*$ & $*$ \\
\hline 2.0 & 0.57 & 0.78 & 0.5 & 0.62 & 0.82 & 1.0 & 0.71 & 0.93 & 4.0 & $*$ & $*$ & $*$ & $*$ & $*$ & $*$ \\
\hline 2.1 & 0.56 & 0.76 & 0.5 & 0.66 & 0.84 & 2.0 & 0.7 & 0.9 & 4.0 & $*$ & $*$ & $*$ & $*$ & $*$ & $*$ \\
\hline 2.2 & 0.55 & 0.75 & 0.5 & 0.64 & 0.86 & 2.0 & 0.67 & 0.87 & 3.0 & * & $*$ & $*$ & $*$ & $*$ & $*$ \\
\hline 2.3 & 0.54 & 0.73 & 0.5 & 0.63 & 0.84 & 2.0 & 0.67 & 0.86 & 4.0 & $*$ & $*$ & $*$ & * & $*$ & $*$ \\
\hline 2.4 & 0.53 & 0.72 & 0.5 & 0.62 & 0.82 & 2.0 & 0.65 & 0.84 & 3.0 & $*$ & $*$ & $*$ & * & $*$ & $*$ \\
\hline 2.5 & 0.52 & 0.7 & 0.5 & 0.61 & 0.81 & 2.0 & 0.64 & 0.83 & 3.0 & * & $*$ & $*$ & $*$ & $*$ & $*$ \\
\hline 2.6 & 0.51 & 0.69 & 0.5 & 0.6 & 0.8 & 2.0 & 0.62 & 0.81 & 3.0 & 0.67 & 0.87 & 10.0 & $*$ & $*$ & $*$ \\
\hline 2.7 & 0.5 & 0.67 & 0.5 & 0.6 & 0.78 & 2.0 & 0.62 & 0.8 & 3.0 & 0.66 & 0.86 & 10.0 & $*$ & $*$ & $*$ \\
\hline 2.8 & 0.5 & 0.67 & 0.5 & 0.59 & 0.77 & 2.0 & 0.61 & 0.79 & 3.0 & 0.65 & 0.85 & 10.0 & $*$ & $*$ & $*$ \\
\hline 2.9 & 0.49 & 0.66 & 0.5 & 0.58 & 0.76 & 2.0 & 0.6 & 0.77 & 3.0 & 0.64 & 0.83 & 10.0 & $*$ & $*$ & $*$ \\
\hline 3.0 & 0.49 & 0.65 & 0.5 & 0.58 & 0.75 & 2.0 & 0.59 & 0.76 & 3.0 & 0.63 & 0.83 & 10.0 & $*$ & $*$ & $*$ \\
\hline
\end{tabular}

Table C.1: Tuning table for $M_{T_{r}}>0.2$. (*): Take the parameters for the precedent case of $\bar{C}(\infty)$. 


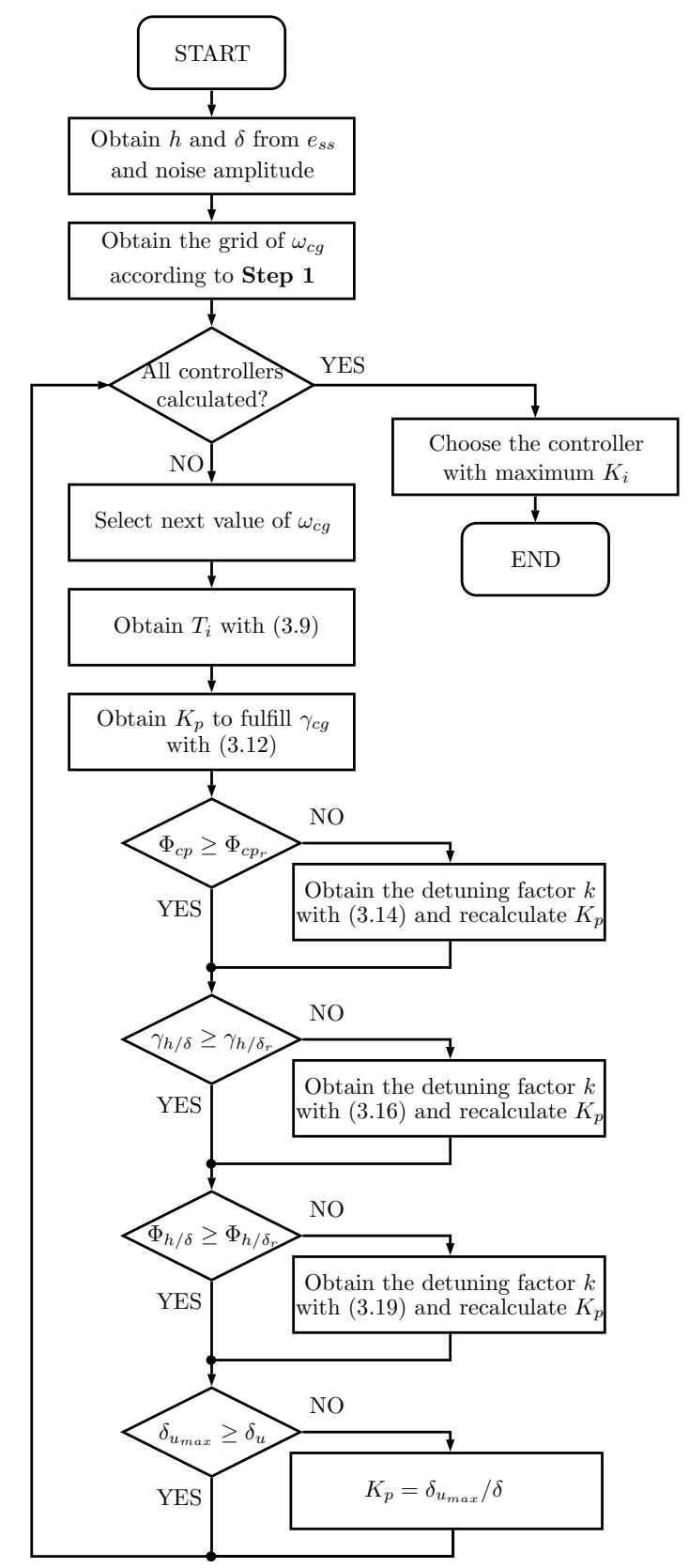

Figure C.1: Flowchart that summarizes the tuning procedure. 



\section{Appendix D}

\section{Supplementary calculus}

\section{D.1 Classical specification dimensionless calculations}

Considering a SISO closed-loop with input disturbance, the relation between the dimensionless error $\bar{E}(\bar{s})$ and the load disturbance $\bar{P}(\bar{s})$ is:

$$
\frac{\bar{E}(\bar{s})}{\bar{P}(\bar{s})}=\frac{\bar{G}(\bar{s})}{1+\bar{G}(\bar{s}) \bar{C}(\bar{s})},
$$

substituting with the given expressions for $\bar{G}(\bar{s})$ and $\bar{C}(\bar{s})$ :

$$
\frac{\bar{E}(\bar{s})}{\bar{P}(\bar{s})}=\frac{e^{-\bar{s}}}{\left[\frac{\tau}{L} \bar{s}+1+\phi_{1}\left(1+\frac{1}{\phi_{2} \bar{s}}+\frac{N \phi_{3} \bar{s}}{N+\phi_{3} \bar{s}}\right) e^{-\bar{s}}\right]},
$$

similarly, for a system with dimensions:

$$
\frac{E(s)}{P(s)}=\frac{K e^{-s L}}{\left[\tau s+1+\phi_{1}\left(1+\frac{1}{L \phi_{2} s}+\frac{N L \phi_{3} s}{N+L \phi_{3} s}\right) e^{-s L}\right]}
$$

Then, the relationship between those two expressions is:

$$
\frac{E(s)}{P(s)}=K \frac{\bar{E}(\bar{s}=s L)}{\bar{P}(\bar{s}=s L)} .
$$

From this expression it can be obtained the effect of a step disturbance of magnitude 1 on $P(s)$ from a dimensionless step of magnitude 1 on $\bar{P}(\bar{s})$ :

$$
E(s)=K \frac{\bar{E}(s L)}{\bar{P}(s L)} P(s)=K \frac{\bar{E}(s L)}{\frac{1}{s L}} \frac{1}{s}=K L \bar{E}(s L) .
$$


Applying over this expression the linearity property of the Laplace transform, the gain $K$ can be extracted. Then, applying the change of scale of the Laplace transform for dealing with the delay:

$$
\mathscr{L}^{-1}\{E(s)\}=K \bar{e}(t / L),
$$

where

$$
\bar{e}(t):=\mathscr{L}^{-1}\{\bar{E}(\bar{s})\}
$$

Being $\overline{I A E}$ the $I A E$ obtained for the dimensionless model:

$$
\overline{I A E}:=\int_{0}^{\infty}|\bar{e}(t)| d t .
$$

Using the expression (D.1) to calculate the index for a generic system:

$$
I A E=\int_{0}^{\infty}|K \bar{e}(t / L)| d t .
$$

Extracting the gain from the integral and performing a variable change $t^{\prime}=t / L$ for solving the integral:

$$
I A E=|K| L \overline{I A E} .
$$

\section{D.2 IE validity in presence of steady-state error}

In the nature of the $E G-C(s)$ control loops is to have a static position error bounded by the switching thresholds of the chosen sampling strategy. Thus, the choice of IE index to select a controller can be questioned because it will tend to infinite. For this reason, a modification of this index has been considered using the sampled error signal $\bar{e}(t)$ instead of the error signal $e(t)$, because it tends to 0 in steady-state, producing the sampled integral error index $I \bar{E}$, defined as:

$$
I \bar{E}=\int_{0}^{\infty} \bar{e}(t) d t
$$

The control action for this kind of non-linear systems is:

$$
u(t)=K_{p} \bar{e}(t)+K_{i} \int_{0}^{t} \bar{e}(\tau) d \tau-K_{d} \frac{d y}{d t},
$$

which for a PI controller:

$$
u(t)=K_{p} \bar{e}(t)+K_{i} \int_{0}^{t} \bar{e}(\tau) d \tau .
$$

Considering a step change at the disturbance input, in steady-state (assuming stability):

$$
u(\infty)=K_{p} \bar{e}(\infty)+K_{i} \int_{0}^{\infty} \bar{e}(t) d t,
$$


as $\bar{e}(\infty)=0$ and taking into account the expression of $I \bar{E}$ :

$$
u(\infty)=K_{i} \int_{0}^{\infty} \bar{e}(t) d t=K_{i} I \bar{E}
$$

From the schema block in Figure 2.1:

$$
(u(\infty)+p(\infty)) G(0)=y(\infty)=-e(\infty),
$$

where $e(\infty)$ is bounded by the steady-state error $e_{s s}$, and, as a unitary step load disturbance is assumed $p(\infty)=1$ :

$$
(u(\infty)+1) G(0) \leq e_{s s},
$$

substituting with the expression of $u(\infty)$ :

$$
\left(K_{i} I \bar{E}+1\right) G(0) \leq e_{s s}
$$

taking into account that the $I E$ caused by a load disturbance is $-1 / K_{i}$ :

$$
I \bar{E} \leq \frac{e_{s s}}{K_{i} G(0)}+I E
$$

from which the difference between both indexes can be found:

$$
I \bar{E}-I E \leq \frac{e_{s s}}{K_{i} G(0)} .
$$

In this last expression, it appears the relation between $I \bar{E}$ and $I E$ indexes. Here, it can be seen that the effect of maximizing $K_{i}$ minimizes the IE index, and makes the difference $I \bar{E}-I E$ minimum. In addition, as the system is assumed stable, the static error is bounded between:

$$
e_{s s} \in\left(-\frac{\delta+h}{2}, \frac{\delta+h}{2}\right)
$$

thus, choosing proper values of $\delta$ and $h$, and maximizing $K_{i}$, the contribution of $e_{s s}$, i.e. the effect of the sampling, can be small enough to consider $I E \approx I \bar{E}$, and thus, it can considered the $I E$ as an appropriate selection index for PI tuning. 



\section{Appendix $\mathbf{E}$}

\section{IEC 61499 Device Models}

As stated in Chapter 7, several additional FBs to enable the communication between devices are needed. These communication FBs are implemented in the Device Model of each device.

In Figure E.1, the device model of the computer's interface is presented. Auxiliary FB to define the interface (FB_LABEL) are presented together with type conversion blocks (REAL2REAL). The communication is carried out by the CLIENT and SERVER FBs, which transmit the data between devices. The set point value is transmitted to the sensor unit with the CLIENT FB

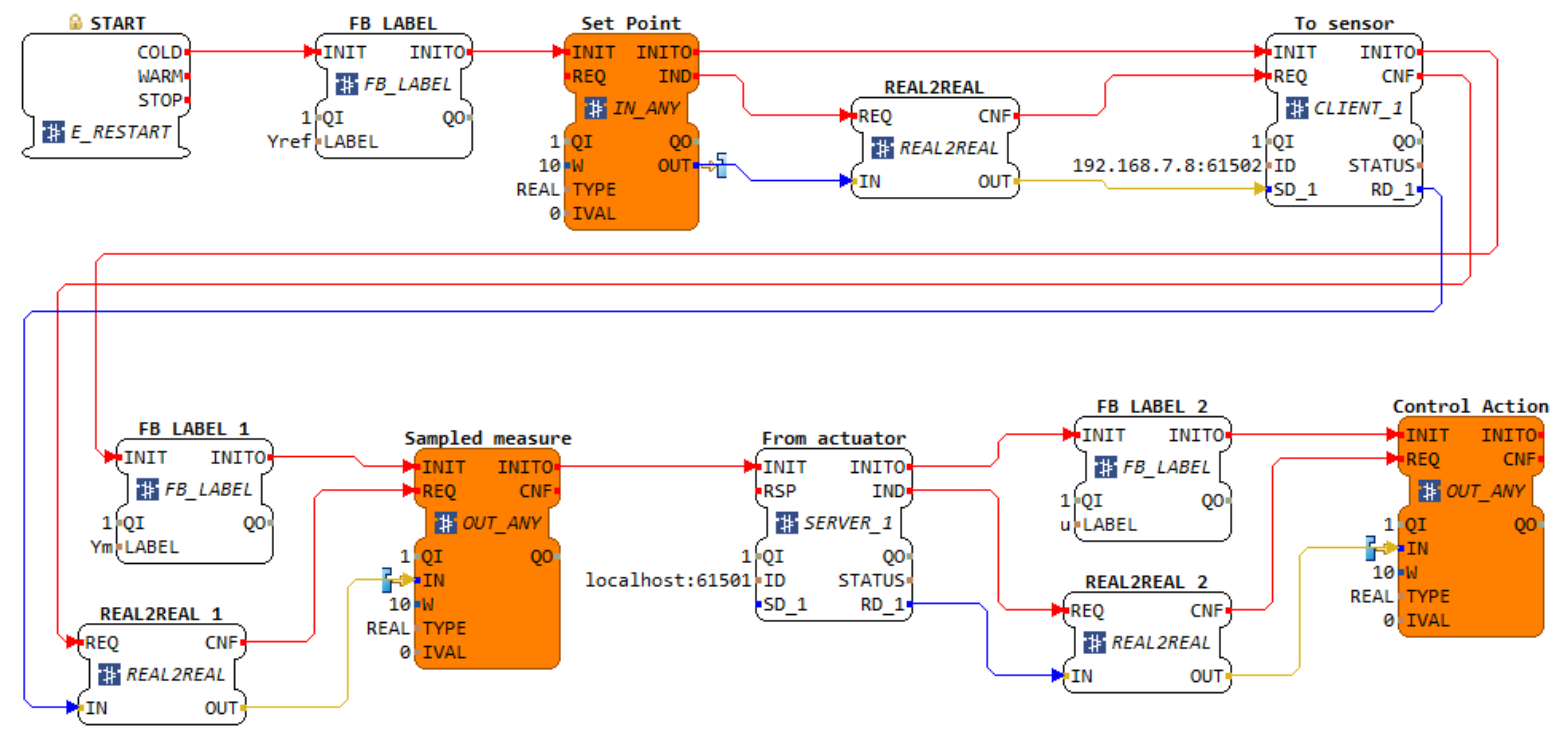

Figure E.1: Device Model of the computer's Interface.

and the control action is received from the SERVER FB.

Similarly to the interface case, the device model of the actuator unit is presented in Figure E.2. In this case, the error value is received from the sensor unit through the SERVER FB, and the CLIENT FB sends the control action to the interface, closing the SERVER-CLIENT pair. 


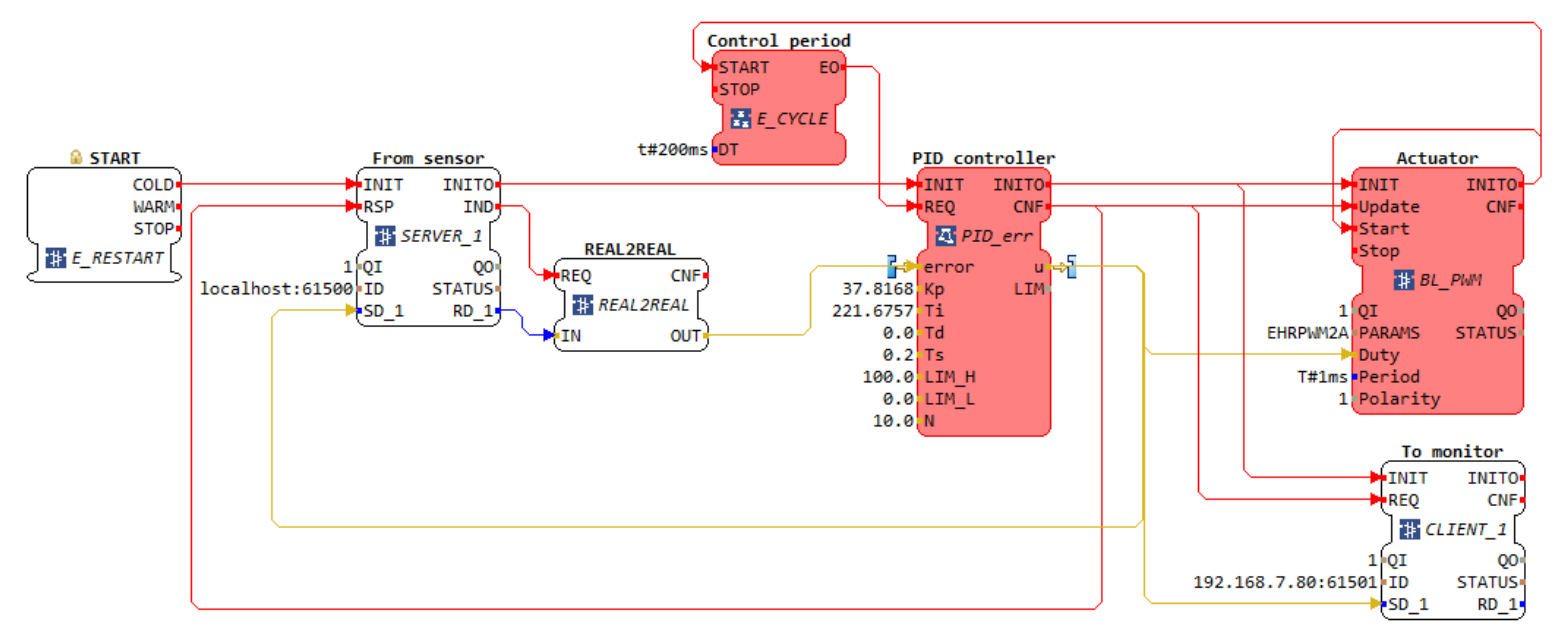

Figure E.2: Device Model of the actuator unit.

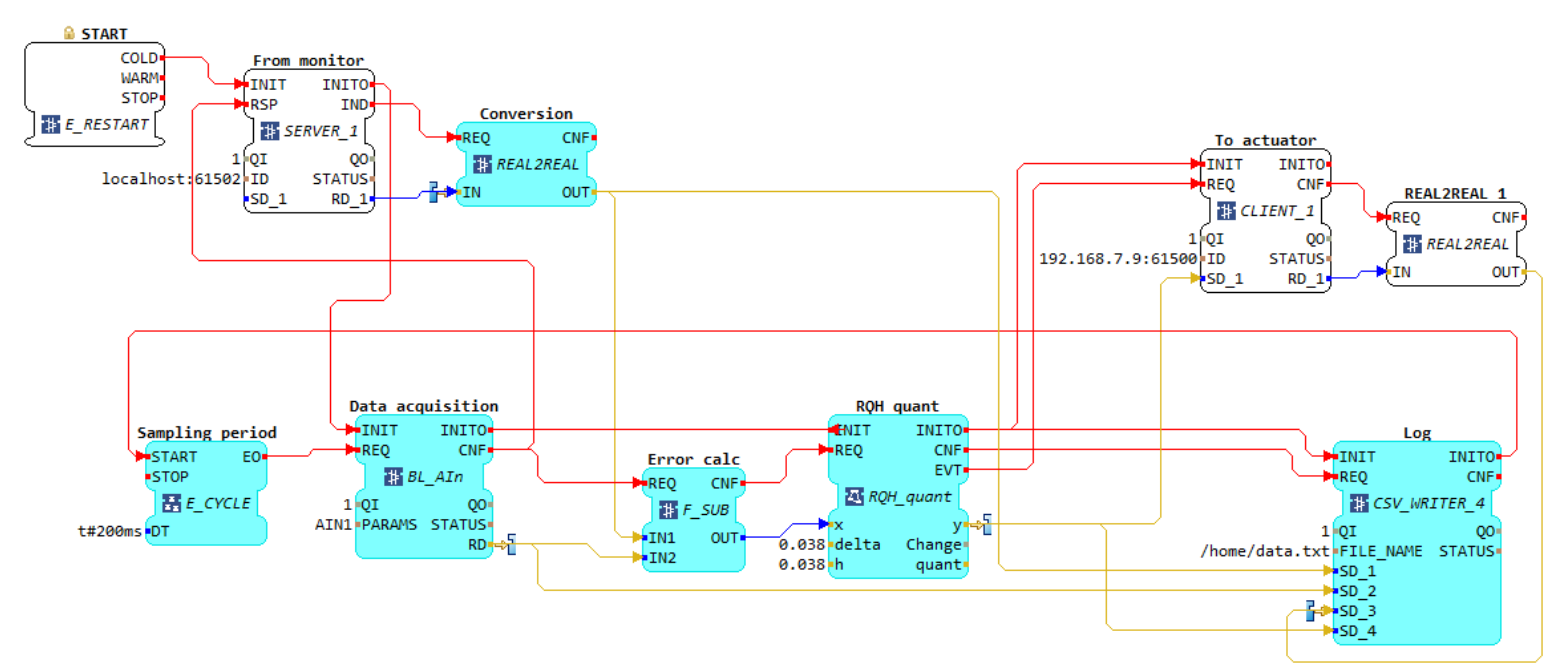

Figure E.3: Device Model of the sensor unit.

In Figure E.3, the device model of the sensor unit is presented. The remaining CLIENTSERVER pairs are completed. A SERVER FB receives the set-point changes from the interface, and the error signal is sent to the actuator unit with a CLIENT FB. It is important to notice that the error measure is sent to the actuator only when a change in the error signal is detected (EVT event in RQH_quant FB), reducing the data traffic between sensor and actuator units. 


\section{Bibliography}

[1] Eclipse 4diac - the open source environment for distributed industrial automation and control systems, 2007-2021.

[2] Ernesto Aranda-Escolástico, María Guinaldo, Rubén Heradio, Jesús Chacón, Héctor Vargas, José Sánchez, and Sebastián Dormido. Event-based control: A bibliometric analysis of twenty years of research. IEEE Access, 8(March):47188-47208, 2020.

[3] Karl-Erik Årzén. A simple event-based PID controller. In Proc. 14th IFAC World Congress, volume 18, pages 423-428, 1999.

[4] Karl J. Åström and Tore Hägglund. Revisiting the Ziegler-Nichols step response method for PID control. Journal of Process Control, 14(6):635-650, sep 2004.

[5] Karl J. Åström and Tore Hägglund. Advanced PID Control. ISA-The Instrumentation, Systems, and Automation Society, 2006.

[6] Karl J. Astrom and Bjorn Wittenmark. Computer-Controlled Systems. Theory and Design. Prentice Hall, third edition, 1997.

[7] Pedro Balaguer-Herrero. Application of dimensional analysis in systems modeling and control design. The Institution of Engineering and Technology, 2013.

[8] M. Beschi, S. Dormido, J. Sanchez, A. Visioli, and L. J. Yebra. Event-based pi plus feedforward control strategies for a distributed solar collector field. IEEE Transactions on Control Systems Technology, 22(4):1615-1622, 2014.

[9] Manuel Beschi, Sebastián Dormido, José Sánchez Moreno, Antonio Visioli, and Luis José Yebra. Event-based PI plus feedforward control strategies for a distributed solar collector field. IEEE Trans. Contr. Sys. Techn., 22:1615-1622, 2014.

[10] Manuel Beschi, Sebastián Dormido, José Sánchez, and Antonio Visioli. Characterization of symmetric send-on-delta PI controllers. Journal of Process Control, 22(10):1930-1945, 2012 .

[11] Manuel Beschi, Sebastián Dormido, José Sánchez, and Antonio Visioli. Tuning of symmetric send-on-delta proportional-integral controllers. IET Control Theory \& Applications, 8:248-259(11), March 2014. 
[12] Manuel Beschi, Andrzej Pawlowski, José Luis Guzmán, Manuel Berenguel, and Antonio Visioli. Symmetric send-on-delta pi control of a greenhouse system. IFAC Proceedings Volumes, 47(3):4411-4416, 2014. 19th IFAC World Congress.

[13] Isabela Birs, Cristina. Muresan, Roxana Both, and Ioan Nascu. A real life implementation of fractional order event based PI control. In 2020 IEEE International Conference on Automation, Quality and Testing, Robotics (AQTR), pages 1-6, 2020.

[14] Isabela Birs, Cristina Muresan, and Clara Ionescu. An event based implementation of a fractional order controller on a non-Newtonian transiting robot. European Control Conference 2020, ECC 2020, pages 1436-1441, 2020.

[15] Isabela Birs, Ioan Nascu, Clara Ionescu, and Cristina Muresan. Event-based fractional order control. Journal of Advanced Research, 25:191 - 203, 2020. Recent Advances in the Fractional-Order Circuits and Systems: Theory, Design and Applications.

[16] Carlos Catalán-Cantero. Modelos y plataforma IEC 61499 adaptados al control distribuido de máquinas herramienta en sistemas de fabricación ágil.

[17] Jesús Chacón, José Sánchez, Antonio Visioli, Luis Yebra, and Sebastián Dormido. Characterization of limit cycles for self-regulating and integral processes with PI control and send-on-delta sampling. Journal of Process Control, 23(6):826-838, 2013.

[18] G. H. Cohen and G. A. Coon. Theoretical consideration of retarded control. Transactions of the ASME, (75):827-834, 1953.

[19] Sebastián Dormido, José Sánchez, and Ernesto Kofman. Muestreo, control y comunicación basados en eventos. Revista Iberoamericana de Automática e Informática Industrial RIAI, $5(1): 5-26,2008$.

[20] Mariagrazia Dotoli, Alexander Fay, Marek Miśkowicz, and Carla Seatzu. An overview of current technologies and emerging trends in factory automation. International Journal of Production Research, 57(15-16):5047-5067, 2019.

[21] George S. Doukas, Kleanthis C. Thramboulidis, and Yannis C. Koveos. Using the function block model for robotic arm motion control. In Control and Automation, 2006. MED'06. 14 th Mediterranean Conference on, pages 1-6. IEEE, 2006.

[22] Sylvain Durand and Nicolas Marchand. An event-based PID controller with low computational cost. In 8th International Conference on Sampling Theory and Applications (SampTA'09), pages Special-session, 2009.

[23] Sylvain Durand and Nicolas Marchand. Further results on event-based PID controller. In Control Conference (ECC), 2009 European, pages 1979-1984. IEEE, 2009.

[24] Laura-Marie Feeney and Martin Nilsson. Investigating the energy consumption of a wireless network interface in an ad hoc networking environment. In Proceedings IEEE INFOCOM 
2001. Conference on Computer Communications. Twentieth Annual Joint Conference of the IEEE Computer and Communications Society (Cat. No. 01CH37213), volume 3, pages $1548-1557$ vol.3, 2001.

[25] Arthur Gelb and Wallace E. Van der Velde. Multiple-input describing functions and nonlinear system design. McGraw-Hill, 1968.

[26] S.F. Graebe and A.J. Isaksson. Derivative filter is an integral part of PID design. IEE Proceedings - Control Theory and Applications, 149(1):41-45, 2002.

[27] Tore Hägglund. The one-third rule for PI controller tuning. Computers \& Chemical Engineering, 127:25-30, 2019.

[28] Tore Hägglund and Karl J. Åström. Revisiting the Ziegler-Nichols tuning rules for PI control. Asian Journal of Control, 4(4):364-380, 2002.

[29] Reinhard Hametner, Georg Schitter, Andreas Voigt, and Alois Zoitl. Implementation guidelines for closed loop control algorithms on PLCs. In Industrial Technology (ICIT), 2013 IEEE International Conference on, pages 1297-1302. IEEE, 2013.

[30] Tien C. Hsia. Analytic Design of Adaptive Sampling Control Law in Sampled-Data Systems. IEEE Transactions on Automatic Control, 19(1):39-42, 1974.

[31] Rolf Isermann. Digital Control Systems, volume 1 Fundamentals, Deterministic Control, chapter Choice of Sample Time for Parameter-optimized Control Algorithms, pages 151-154. Springer-Verlag Berlin Heidelberg GmbH, second edition, 1989.

[32] Karl-Heinz John and Michael Tiegelkamp. IEC 61131-3: programming industrial automation systems: concepts and programming languages, requirements for programming systems, decision-making aids. Springer Science \& Business Media, 2010.

[33] Eliahu I. Jury. Sampled-data Control Systems. John Wiley \& Sons, Inc., New York, 1958.

[34] Birgitta Kristiansson and Bengt Lennartson. Robust Tuning of PI and PID Controllers. Using derivative action despite the sensor noise. IEEE Control Systems Magazine, (February):55-68, 2006.

[35] Nikolai Mitrofanovich Krylov and Nikolai Nikolaevich Bogoliubov. Introduction to nonlinear mechanics. Princeton university press, 1949.

[36] Benjamin C. Kuo. The z-transform describing function for nonlinear sampled-data control systems. Proceedings IRE, 48(5):941-942, 1960.

[37] Benjamin C. Kuo. Analysis and synthesis of sampled-data control systems. 1963.

[38] Jan Lunze. Event-based control: Introduction and survey. In M. Miskowicz, editor, EventBased Control and Signal Processing, pages 3-20. Boca Raton: CRC Press, 2015. 
[39] Anca Maxim, Dana Copot, Cosmin Copot, and Clara M. Ionescu. The 5w's for control as part of industry 4.0: Why, what, where, who, and when-A PID and MPC control perspective. Inventions, 4(1), 2019.

[40] O. P. McNamara and D. P. Atherton. Limit cycles in non linear sampled data systems. IFAC Proceedings Volumes, 17(2):507-512, 1984.

[41] Oscar Miguel-Escrig and Julio-Ariel Romero-Pérez. Implementation and evaluation of event-based PID in the IEC-61499 standard. In 2018 IEEE 23rd International Conference on Emerging Technologies and Factory Automation (ETFA), volume 1, pages 574-580. IEEE, 2018.

[42] Oscar Miguel-Escrig and Julio-Ariel Romero-Pérez. Regular quantisation with hysteresis: a new sampling strategy for event-based PID control systems. IET Control Theory \& Applications, 2020.

[43] Oscar Miguel-Escrig, Julio-Ariel Romero-Pérez, and Roberto Sanchis-Llopis. New robustness measure for a kind of event-based PID. IFAC-PapersOnLine, 51(4):781-786, 2018.

[44] Oscar Miguel-Escrig, Julio-Ariel Romero-Pérez, and Roberto Sanchis-Llopis. Tuning PID controllers with symmetric send-on-delta sampling strategy. Journal of the Franklin Institute, 357(2):832-862, 2020.

[45] Marek Miskowicz. Send-on-delta concept: An event-based data reporting strategy. Sensors, 6(1):49-63, 2006.

[46] Cristina Muresan, Isabela Birs, and Eva H. Dulf. Event-based implementation of fractional order IMC controllers for simple FOPDT processes. Mathematics, 8(8):1378, Aug 2020.

[47] Amir Nassirharand. Computer-aided Nonlinear Control System Design: Using Describing Function Models. Springer London, 2012.

[48] Aidan O'Dwyer. Handbook of PI and PID controller tuning rules. Imperial College Press, 2nd edition, 2006.

[49] Andrzej Pawlowski, Manuel Beschi, José L. Guzmán, Antonio Visioli, Manuel Berenguel, and Sebastián Dormido. Application of SSOD-PI and PI-SSOD event-based controllers to greenhouse climatic control. ISA Transactions, 65:525 - 536, 2016.

[50] Joerns Ploennigs, Volodymyr Vasyutynskyy, and Klaus Kabitzsch. Comparative study of energy-efficient sampling approaches for wireless control networks. Industrial Informatics, IEEE Transactions on, 6(3):416 -424, aug. 2010.

[51] Esteban Querol-Dolz, Julio-Ariel Romero-Pérez, Julio Serrano-Mira, and Roberto SanchisLlopis. Evaluation of closed loop control applications using different event management strategies under IEC 61499. In Event-based Control, Communication, and Signal Processing (EBCCSP), 2016 Second International Conference on, pages 1-8. IEEE, 2016. 
[52] E. Rodríguez-Miranda, M. Beschi, J. L. Guzmán, M. Berenguel, and A. Visioli. Application of a symmetric-send-on-delta event-based controller for a microalgal raceway reactor. In 2019 18th European Control Conference (ECC), pages 3132-3137, 2019.

[53] Enrique Rodríguez-Miranda, Manuel Beschi, José Luis Guzmán, Manuel Berenguel, and Antonio Visioli. Daytime/nighttime event-based pi control for the ph of a microalgae raceway reactor. Processes, 7(5), 2019.

[54] J. A. Romero, R. Sanchis, and E. Arrebola. Experimental study of event based pid controllers with different sampling strategies. application to brushless dc motor networked control system. In 2015 XXV International Conference on Information, Communication and Automation Technologies (ICAT), pages 1-6, 2015.

[55] Julio-Ariel Romero-Pérez, Néstor J. Pascual, Ignacio Peñarrocha-Alós, and Roberto Sanchis-Llopis. Event-based PI controller with adaptive thresholds. In Ultra Modern Telecommunications and Control Systems and Workshops (ICUMT), 2012 4th International Congress on, pages 219-226. IEEE, 2012.

[56] Julio-Ariel Romero-Pérez and Roberto Sanchis-Llopis. A new method for tuning PI controllers with symmetric send-on-delta sampling strategy. ISA Transactions, 64:161 - 173, 2016 .

[57] Julio-Ariel Romero-Pérez and Roberto Sanchis-Llopis. Analysis of a simple rule for tuning SSOD based PIDs. In Event-based Control, Communication, and Signal Processing (EBCCSP), 2016 Second International Conference on, pages 1-8. IEEE, 2016.

[58] Julio-Ariel Romero-Pérez and Roberto Sanchis-Llopis. Tuning and robustness analysis of event-based PID controllers under different event generation strategies. International Journal of Control, pages 1-38, 2017.

[59] Julio-Ariel Romero-Pérez, Roberto Sanchis-Llopis, and Elena Arrebola. Experimental study of event based PID controllers with different sampling strategies. Application to brushless DC motor networked control system. In Information, Communication and Automation Technologies (ICAT), 2015 XXV International Conference on, pages 1-6, Oct 2015 .

[60] Julio-Ariel Romero-Pérez, Roberto Sanchis-Llopis, and Ignacio Peñarrocha-Alós. A simple rule for tuning event-based PID controllers with symmetric send-on-delta sampling strategy. In Proceedings of the 2014 IEEE Emerging Technology and Factory Automation (ETFA), pages 1-8, Sept 2014.

[61] Ángel Ruiz, Manuel Beschi, Antonio Visioli, Sebastián Dormido, and Jorge E. Jiménez. A unified event-based control approach for FOPTD and IPTD processes based on the filtered Smith predictor. Journal of the Franklin Institute, 354(2):1239-1264, 2017.

[62] Tariq Samad. A survey on industry impact and challenges thereof. IEEE Control Systems, 37(1):17-18, 2017. 
[63] José Sánchez, María Guinaldo, Sebastián Dormido, and Antonio Visioli. Validity of continuous tuning rules in event-based PI controllers using symmetric send-on-delta sampling: An experimental approach. Computers \& Chemical Engineering, page 106878, 2020.

[64] José Sánchez, María Guinaldo, Antonio Visioli, and Sebastián Dormido. Enhanced eventbased identification procedure for process control. Industrial \& Engineering Chemistry Research, 57(21):7218-7231, 2018.

[65] José Sánchez, María Guinaldo, Antonio Visioli, and Sebastián Dormido. Identification and tuning methods for PI control systems based on symmetric send-on-delta sampling. International Journal of Control, Automation and Systems, 17(11):2784-2795, 2019.

[66] Roberto Sanchis-Llopis, Julio-Ariel Romero-Pérez, and Pedro Balaguer-Herrero. Tuning of PID controllers based on simplified single parameter optimisation. International Journal of Control, 83(9):1785-1798, 2010.

[67] Necip Sayiner, Henrik V. Sorensen, and Thayamkulangara R. Viswanathan. A level-crossing sampling scheme for A/D conversion. IEEE Transactions on Circuits and Systems II: Analog and Digital Signal Processing, 43(4):335-339, 1996.

[68] Sigurd Skogestad. Simple analytic rules for model reduction and PID controller tuning. Journal of process control, 13(4):291-309, 2003.

[69] Jean-Jacques E. Slotine and Weiping Li. Appied Nonlinear Control. Prentice Hall, 1991.

[70] José Sánchez, María Guinaldo, Antonio Visioli, and Sebastián Dormido. Identification of process transfer function parameters in event-based PI control loops. ISA Transactions, $75: 157-171,2018$.

[71] Thomas Strasser, Franz Auinger, and Alois Zoitl. Development, implementation and use of an IEC 61499 function block library for embedded closed loop control. In Industrial Informatics, 2004. INDIN'04. 2004 2nd IEEE International Conference on, pages 594-599. IEEE, 2004.

[72] Li Sun, Donghai Li, and Kwang Y. Lee. Optimal disturbance rejection for PI controller with constraints on relative delay margin. ISA Transactions, 63:103-111, 2016.

[73] José A. Tenreiro-Machado. Fractional order describing functions. Signal Processing, 107:389 - 394, 2015. Special Issue on ad hoc microphone arrays and wireless acoustic sensor networks Special Issue on Fractional Signal Processing and Applications.

[74] Yannis Tsividis. Event-driven data acquisition and digital signal processing-A tutorial. IEEE Transactions on Circuits and Systems II: Express Briefs, 57(8):577-581, 2010.

[75] Yakov Z. Tsypkin. Relay control systems. Cambridge University Press, 1984.

[76] Volodymyr Vasyutynskyy and Klaus Kabitzsch. Time constraints in PID controls with send-on-delta. IFAC Proceedings Volumes, 42(3):48-55, 2009. 
[77] Volodymyr Vasyutynskyy and Klaus Kabitzsch. A comparative study of PID control algorithms adapted to send-on-delta sampling. In Industrial Electronics (ISIE), 2010 IEEE International Symposium on, pages 3373-3379. IEEE, 2010.

[78] J.C. West, J.L. Douce, and R.K. Livesley. The dual-input describing function and its use in the analysis of non-linear feedback systems. Proceedings of the IEE-Part B: Radio and Electronic Engineering, 103(10):463-473, 1956.

[79] John G. Ziegler and Nathaniel B. Nichols. Optimum settings for automatic controllers. trans. ASME, 64(11), 1942.

[80] Alois Zoitl and Robert Lewis. Modelling control systems using IEC 61499, volume 95. IET, 2014. 




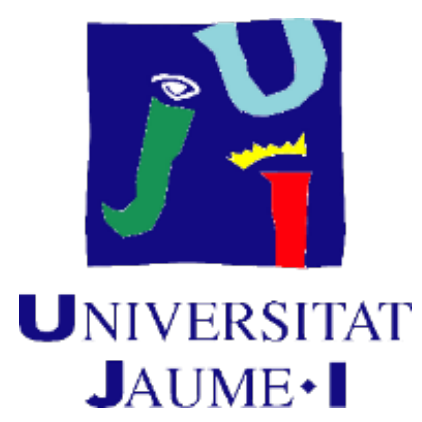

